\title{
LIPID-LOWERING THERAPY
}

IN

\section{CALCIFIC AORTIC STENOSIS}

BY

Sarah Joanna Cowell

A thesis presented for the degree of Doctor of Medicine at the

University of Edinburgh 


\section{ABSTRACT}

Background Calcific aortic stenosis is the commonest valvular heart condition seen in the western world and appears to be the result of an active inflammatory process closely resembling atherosclerosis. I hypothesised that (a) risk factors for atherosclerosis would predict, and (b) lipid-lowering therapy would retard, disease progression and clinical outcome in patients with calcific aortic stenosis.

Objectives (i) To compare the magnitude and reproducibility of measures of valvular stenosis and calcification, (ii) to determine the effect of intensive lipidlowering therapy on disease progression and clinical outcome, and (iii) to describe predictors of disease progression and clinical outcome in patients with aortic stenosis.

Methods These issues were addressed in the context of a randomised controlled trial of which I was study co-ordinator. In the Scottish Aortic stenosis and Lipid-lowering Therapy, Impact on REgression (SALTIRE) trial, 155 patients aged $68 \pm 11$ years (range 34-85) with aortic valve stenosis were randomised, and underwent helical computed tomography (CT) and Doppler echocardiography. Seventy-seven patients were assigned to atorvastatin $80 \mathrm{mg}$ daily and 78 to matched placebo over a median period of 25 months. Of the 155 patients, 102 had detectable coronary artery calcification on CT with 48 of these patients being randomised to atorvastatin and 54 to placebo.

Results (i) Stenosis severity: Doppler echocardiography demonstrated a mean aortic jet velocity of $3.45 \pm 0.66$ metres per second $(\mathrm{m} / \mathrm{s})$ and a peak gradient of $49 \pm 11$ millimetres of mercury $(\mathrm{mmHg})$. Computed tomography and Doppler echocardiography showed good reproducibility. The median aortic valve calcium (AVC) score was 5858 Hounsfield units (HU) (interquartile range, 1555-14596) and this positively correlated with aortic jet velocity and peak gradient $(\mathrm{r}=0.54, \mathrm{p}<0.0001$ for both). (ii) Disease progression: Aortic jet velocity increased by $0.199 \pm 0.210$ $\mathrm{m} / \mathrm{s}$ per year in the atorvastatin group and $0.203 \pm 0.208 \mathrm{~m} / \mathrm{s}$ per year in the placebo group ( $\mathrm{p}=0.95$; adjusted mean difference, $0.002 ; 95 \%$ confidence interval (CI)), $(-0.066$ to $+0.070 \mathrm{~m} / \mathrm{s} / \mathrm{yr})$. Progression in valvular calcification was $22 \pm 21 \%$ per year in the atorvastatin group, and $22 \pm 20 \%$ per year in the placebo group $(p=0.93$; ratio of post-treatment AVC score, $0.998 ; 95 \%$ CI, 0.947 to 1.050$)$. The rate of change in coronary artery calcification was $26 \%$ per year $(0.234 \pm 0.037 \log$ arbitrary units/yr $(\operatorname{LogAU} / \mathrm{yr}) ; \mathrm{n}=39)$ in the atorvastatin group and $18 \%$ per year $(0.167 \pm$ $0.034 \mathrm{LogAU} / \mathrm{yr} ; \mathrm{n}=49$ ) in the placebo group: geometric mean difference of $+7 \%$ per year $(95 \% \mathrm{CI},-3 \%$ to $+18 \%$; $=0.18)$. (iii) Lipid-lowering: Atorvastatin reduced serum low-density lipoprotein (LDL) cholesterol $(-53 \% ; \mathrm{p}<0.001)$ and C-reactive protein $(\mathrm{CRP})(-49 \% ; \mathrm{p}<0.001)$ concentrations whilst there was no change with placebo $(-7 \%$ and $+17 \% ; \mathrm{p}>0.95$ for both). There was no correlation between serum LDL concentrations and the progression of aortic stenosis or coronary calcification. (vi) Predictors of disease progression and outcome: Aortic valve disease progression was predicted by age, sex, height, hypertension, serum brain natriuretic peptide (BNP) concentration, and baseline valve disease severity. Clinical outcome was predicted by baseline and rate of progression of aortic stenosis severity and serum 
Conclusions Calcification of the aortic valve is closely associated with the severity of aortic stenosis with heavy calcification suggesting the presence of severe aortic stenosis that requires urgent cardiological assessment. In contrast to observational studies, intensive lipid-lowering therapy does not halt the progression or induce regression of aortic stenosis or coronary artery calcification. However, our studies cannot exclude a small reduction in disease progression or a significant reduction in major clinical end-points. Long-term, large scale, randomised, controlled trials are needed to establish the role of statin therapy in patients with calcific aortic stenosis. The major predictors of disease progression and clinical outcome remain baseline measures of disease severity; namely aortic jet velocity, aortic valve calcification and serum BNP concentration. With the exception of hypertension, the presence of atherosclerotic risk factors and vascular disease are not predictive. Our findings suggest that atherogenesis does not provide a major contribution to the progression of aortic stenosis. 
To Megan and Callum 


\title{
CONTENTS
}

\author{
Abstract \\ Contents
}

Abbreviations

Declaration

Acknowledgements

CHAPTER 1:

INTRODUCTION: CALCIFIC AORTIC STENOSIS

1.1 Introduction

1.2 Epidemiology

1.3 Natural history

1.4 Pathology of calcific aortic stenosis

1.5 Clinical presentation

1.6 Investigations

1.7 Management of calcific aortic stenosis

1.8 Conclusion

1.9 Aims and hypotheses 
CHAPTER 2:

Materials ANd Methods

2.1 Introduction

2.2 The SALTIRE trial

2.3 Biochemical variables

2.4 Echocardiography

2.5 Computed tomography

2.6 Pulse wave analysis

2.7 Data analysis and statistics

CHAPTER 3:

AORTIC VALVE CALCIFICATION ON COMPUTED TOMOGRAPHY PREDICTS THE SEVERITY OF AORTIC STENOSIS

3.1 Summary

3.2 Introduction

3.3 Methods

3.4 Results

3.5 Discussion

CHAPTER 4:

INTENSIVE LIPID-LOWERING THERAPY DOES NOT HALT

THE PROGRESSION OF CALCIFIC AORTIC STENOSIS

4.1 Summary 


\subsection{Introduction}

\subsection{Methods}

$4 \quad$ Results

4.5 Discussion

CHAPTER 5:

Progressive CORONARY CALCIFICATION DESPITE INTENSIVE

LIPID-LOWERING THERAPY: A RANDOMISED CONTROLLED TRIAL

5.1 Summary

5.2 Introduction

5.3 Methods

5.4 Results

5.5 Discussion

CHAPTER 6:

Predictors OF DISEASE PROGRESSION AND CLINICAL

OUTCOME IN PATIENTS WITH CALCIFIC AORTIC STENOSIS
6.1 Summary
6.2 Introduction
6.3 Methods
6.4 Results
6.5 Discussion 
CHAPTER 7:

$135-153$

CONCLUSIONS AND FUtURE diRECTIONS

7.1 Conclusions

7.2 Future directions and clinical implications

7.3 Summary

REFERENCES

154-171

APPENDIX

172

Publications arising from or relevant to this thesis 


\section{ABBREVIATIONS}

ACE

ANOVA

AS

AU

AVA

AVC

AVR

BNP

CI

CRP

CT

CVA

HDL

HMG CoA

HsCRP

HU

ICAM-1

ITT

LAD

LDL

Lp (a)
Angiotensin-converting enzyme

Analysis of variance

Aortic stenosis

Arbitrary units

Aortic valve area

Aortic valve calcium

Aortic valve replacement

Brain natriuretic peptide

Confidence interval

C-reactive protein

Computed tomography

Cerebrovascular accident

High-density lipoprotein

Hydroxymethylglutaryl coenzyme A

Highly sensitive C-reactive protein

Hounsfield units

Intercellular adhesion molecule-1

Intention-to-treat

Left anterior descending

Low-density lipoprotein

Lipoprotein a 
LVOTD

MR

NYHA

PLSD

PWA

SALTIRE

SD

SE

VCAM-1
Left ventricular outflow tract diameter

Magnetic resonance

New York Heart Association

Protected least squares difference

Pulse wave analysis

The Scottish Aortic stenosis and Lipid lowering

Therapy Impact on REgression trial

Standard deviation

Standard error

Vascular cell adhesion molecule-1 


\section{DECLARATION}

This thesis represents research undertaken in the Department of Cardiology at the Royal Infirmary and Western General Hospital in Edinburgh. The substantial part of the work described has been my own and carried out during the period between 2000 and 2004 whilst I was a Clinical Research Fellow. As the SALTIRE trial study coordinator I was involved in all aspects of the study including ethics submissions, recruitment and randomisation, data collation and analysis, and writing for publication. I have received help and advice from many colleagues, and they have been formally acknowledged. The thesis has not been accepted in any previous applications for a degree and all sources of information have been acknowledged. The work has been published in peer-reviewed journals: see Bibliography.

Sarah Joanna Cowell

$31^{\text {st }}$ March 2007 


\section{ACKNOWLEDGEMENTS}

As a research fellow I have been fortunate to work with many people who have supported, advised and encouraged me. In particular I would like to thank my research supervisors;

Dr Nicholas Boon, for agreeing to appoint me as research fellow to co-ordinate the SALTIRE trial, and guide me through this fantastic opportunity, that has provided me with outstanding research experience.

Dr Andrew Elder for his support and guidance over many years in both clinical medicine and research, and in particular for encouraging me to start writing at an early stage during the research fellowship.

During four years in clinical research I worked with a large team of medical, nursing and allied health professionals, all of whom contributed to the smooth running and successful outcome of the trial. I am grateful to the entire SALTIRE trial research team, which included; Lorraine Anderson, Laura Flint, Maureen O'Donnell, Jean Cunningham, Corinne Bell, Judith Burton, Margaret Henderson, Margaret Bland, Audrey White, and the Wellcome Trust Clinical Research Facility research nurses (Sharon Cameron, Hayley Cuthbertson, Dawn Lyle, Finny Paterson, Karen Paterson, Heather Spence, Julie Tickner). In particular I am grateful to Laura Flint who throughout the trial provided guidance, practical and moral support, and who supervised the research team during my maternity leave absence. Thanks also go to Lorraine Anderson whose rapport with patients and staff, and personal dedication ensured the smooth running of this trial; to Dolly (Audrey White) for her patience 
and meticulous attention to detail in all echocardiography assessments, without whom this the trial would not have succeeded; to Judith Burton and Margaret Henderson for providing a weekend computed tomography service specifically for the SALTIRE trial, and for providing support as well as a local tourist information service to patients (most of whom travelled a considerable distance for their CT scans); and to Jean Cunningham for secretarial support with data collation and the preparation of this thesis, without whose constant encouragement many more deadlines would have passed.

I acknowledge the assistance of clinical research fellow colleagues Nicholas Cruden and Simon Robinson, both of whom committed considerable time and effort in assisting with the final stages of patient recruitment, and who supervised patient care during my maternity leave.

I greatly appreciate the guidance of John Reid and his considerable commitment in scoring the computed tomography data. I am grateful to Dave Northridge for his guidance with the echocardiography data collection, and to Robin Prescott for statistical guidance as well as conducting the complex statistical analyses. Thanks also go to Peter Bloomfield for clinical advice and data monitoring, and the assistance of Emma Houslay with the analysis of the coronary computed tomography data. 
I acknowledge help received from Andrew Heed and Brian Booth with labelling, storing and dispensing study medications, and from Thorsten Forster with the randomisation software.

I am very grateful to all patients who participated in the SALTIRE trial, and the British Heart Foundation without whom this research would not have been possible.

Finally I am indebted to my husband without whose support, guidance, constant nagging and encouragement this research and thesis would never have been successfully completed. I am grateful to my children Megan and Callum for their patience in allowing me to finish this thesis. 


\section{CHAPTER 1}

\section{CALCIFIC AORTIC STENOSIS}

Based on

Cowell SJ, Newby DE, Boon NA, Elder AT.

Calcific aortic stenosis: Same old story? Age Ageing 2004;33(6):538-44. 


\subsection{INTRODUCTION}

Aortic stenosis is the commonest adult heart valve condition seen in the western world. It may be the result of a congenital lesion, or it can arise following rheumatic fever. More commonly it occurs as a result of calcification of a congenitally bicuspid or a trileaflet aortic valve (calcific aortic stenosis). Over the last 30-50 years, its diagnosis and management has been revolutionised by the development of invasive (cardiac catheterisation) and non-invasive (echocardiography) haemodynamic assessments as well as potentially curative cardiac surgery. Recent insights have been made into the pathogenesis of calcific aortic stenosis, resulting in speculation that the disease mimics atherosclerosis and progression could be delayed or prevented by the use of lipid-lowering therapy. If confirmed, such pharmacological therapy may have the potential to reduce the need for aortic valve surgery.

\subsection{EPIDEMIOLOGY}

Calcific aortic stenosis was first documented in 1904 [Monckeburg 1904] and at that time was regarded as uncommon. In the $19^{\text {th }}$ century, calcific aortic stenosis was not recognised as a clinical entity since pathological studies revealed only cusp thickening and sclerosis [Osler 1886]. As a result, aortic valve sclerosis (thickening without stenosis) and aortic valve stenosis were regarded as different pathological conditions for many decades. Recent evidence, however, suggests that they represent different stages of the same disease process [Stewart et al 1997; Cosmi et al 2002; Faggiano et al 2003]: sclerosis arising from the development of valvular calcific 
lesions that progress slowly over several decades before ultimately causing aortic stenosis [Mensah and Friesinger 1996]. The current prominence of calcific aortic valve disease is likely to represent increased human longevity together with the decline in rheumatic valvular heart disease.

Aortic valve sclerosis is present in $20-30 \%$ of individuals over 65 years and $48 \%$ over 85 years [Otto et al 1999], and aortic stenosis in $2 \%$ and $4 \%$ respectively [Lindroos et al 1993; Stewart et al 1997; Otto et al 1999]. Calcific sclerosis and valvular stenosis occur in patients with both a normal tricuspid aortic valve as well as in those with a bicuspid valve. The prevalence of bicuspid aortic valves is difficult to determine but is estimated to affect $1-2 \%$ of the general population [Ward 2000]. Up to $70 \%$ of patients with a bicuspid aortic valve develop valvular stenosis [Ward 2000] and will typically require aortic valve replacement (AVR) one to two decades earlier in life (fifth to sixth decade) than in those with a tricuspid aortic valve.

\subsection{NATURAL HISTORY}

Prior to the introduction of haemodynamic assessment and cardiac surgery, the natural history of aortic stenosis was described by its clinical presentation. Calcific aortic stenosis is a gradually progressive disease, characterised by a long asymptomatic phase lasting several decades, followed by a shorter symptomatic phase usually associated with severe narrowing of the aortic valve orifice.

The outlook for patients with asymptomatic aortic stenosis is generally good and closely matches that of life table estimates for age and sex matched controls 
[Pellikka et al 1990]. A striking feature of aortic stenosis is that the prognosis changes dramatically with the onset of symptoms in association with severe outflow obstruction: a 2-year survival rate of $50 \%$. Although few studies specifically assessed the influence of age, patients over the age of seventy have a worse prognosis with 2and 3-year survival rates of $37 \%$ and $25 \%$ respectively [O'Keefe et al 1987]. The prognosis also depends upon the clinical presentation with a mean survival of 3 years

for those presenting with angina and syncope, 2 years with the onset of breathlessness, and as little as 1 year in those who develop overt left ventricular failure [Ross and Braunwald 1968; Aronow et al 1993].

\subsubsection{OTHER CARDIOVASCULAR EVENTS}

Despite the favourable outlook in those patients with mild asymptomatic disease, there is an increased risk of cardiovascular events unrelated to the aortic valve disease. Otto and colleagues demonstrated that, in patients with aortic sclerosis, there is a $50 \%$ increased risk of myocardial infarction and cardiovascular death even in the absence of significant outflow tract obstruction [Otto et al 1999]. The Helsinki Ageing Study also suggested that patients with moderate to severe aortic stenosis had higher all cause and cardiovascular mortality irrespective of associated symptoms. In particular, a higher rate of stroke related death was noted although the majority of these patients had atrial fibrillation [Iivanainen et al 1996]. 


\subsection{PATHOLOGY OF CALCIFIC AORTIC STENOSIS}

For many decades, calcific aortic stenosis has been attributed to prolonged "wear and tear" and age-associated valvular degeneration. Contrary to this supposition, however, is the absence of aortic valve calcification or stenosis on echocardiography in a third of individuals over the age of 80 [Lindroos et al 1993]. Recent evidence suggests that calcific aortic stenosis may result from an active inflammatory process involving biochemical, humoral and genetic factors.

\subsubsection{Histology}

Normal aortic valve leaflets are macroscopically smooth, thin and opalescent, with clearly defined tissue layers at a microscopic level and very few cells [Olssson et al 1994]. Increasing age gives rise to non-specific thickening of the tips of the valve leaflets, with an increase in the number of adipose cells and thinning of tissue layers [Otto et al 1994]. In calcific aortic stenosis, there is characteristic leaflet thickening, with irregular nodular masses on the aortic aspect of the valve. Microscopic assessment of both mild and severely affected valves reveals endothelial and basement membrane disruption, with underlying subendothelial thickening. The lesion itself contains disorganised collagen fibres, chronic inflammatory cells, lipoproteins, lipid, extracellular bone matrix proteins and bone mineral [Olsson et al 1994; Otto et al 1994]. 


\subsubsection{Pathogenesis}

The histological features described closely resemble those seen in atherosclerosis and are strongly suggestive of chronic inflammation. In calcific aortic stenosis, the factors initiating the inflammatory process have not been identified but mechanical injury to the endothelium is thought to pave the way for subsequent inflammation. This concept is supported by the pattern of aortic valve cusp involvement that corresponds to areas of low shear and high tensile stress: namely the aortic surface of the leaflets and predilection for the non-coronary cusp [Thubrikar et al 1986; Cujec and Pollick 1988; Yearwood et al 1989; Otto 2002]. Congenitally bicuspid aortic valves are less efficient than tricuspid valves at distributing mechanical stress and this may account for their predilection to develop rapidly progressive stenosis [Beppu et al 1993].

\subsubsection{Role of lipids}

Endothelial injury or disruption may allow circulating lipids to enter the valvular interstitial tissue [O'Brien et al 1996] and accumulate in areas of calcification and inflammation [O'Brien et al 1996; Olsson et al 1999]. The lipoproteins implicated in atherogenesis, including low-density lipoprotein (LDL) and lipoprotein (a) (Lp (a)), are present in early aortic valve lesions [O'Brien et al 1996] and undergo oxidative modification [Olsson et al 1999]. These oxidised lipoproteins are highly cytotoxic [Chisholm 1991] and capable of stimulating inflammatory activity [Berliner et al 1990; Rajavashisth et al 1990] and mineralisation [Hirsch et al 1993; Sarig et al 1994; Parhami et al 1997]. 


\subsubsection{Inflammation}

Both macrophages and activated $\mathrm{T}$ lymphocytes are present in the early and advanced lesions of congenitally bicuspid [Wallby et al 2002] and tricuspid aortic valves [Olsson et al 1994; Otto et al 1994]. Migration of these effector inflammatory cells appears to be mediated through increased endothelial expression of cellular adhesion molecules such as E-selectin, intercellular adhesion molecule-1 (ICAM-1 and vascular cell adhesion molecule-1 (VCAM-1) [Ghaisas et al 2000; Müller et al 2000]. Once recruited into the subendothelium, the inflammatory cells release enzymes, such as matrix metalloproteinases, that cause degradation of collagen, elastin and proteoglycans within the aortic valve cusps [Edep et al 2000].

\subsubsection{Calcification}

Mineralisation is a characteristic of both atherosclerotic and aortic valve lesions, and arises in close proximity to areas of inflammation. It is a prominent feature in calcific aortic stenosis and has been demonstrated in early [Otto et al 1994] as well as advanced lesions [Mohler et al 2001]. Surgically excised valves have even revealed areas of mature lamellar bone, haemopoietic marrow and bone remodelling [Mohler et al 2001]. Several features suggest the presence of an active highly regulated process closely resembling developmental bone formation [Boström et al 1995; Demer 1995].

The initiation of mineralisation (nucleation) may be stimulated by the presence of cellular degradation products following apoptosis [Kockx and Herman 1998] or by the presence of oxidised lipids [Olsson et al 1999; Mohler et al 2001]. In vitro 
studies of cultured explants of stenotic valves have identified cells with osteoblastic characteristics capable of phenotypic differentiation and spontaneous calcification [Mohler et al 1999]. Their origin is unknown but they may be derived from a pool of circulating immature pluripotent mesenchymal cells [Prockop 1997]. These osteogenic cells or "calcifying valvular cells" express and produce a variety of regulatory bone matrix proteins including osteopontin [O'Brien et al 1995; Mohler et al 1997] and bone morphogenetic protein [Mohler et al 2001].

\subsubsection{SIMILARITIES AND DIFFERENCES WITH ATHEROSCLEROSIS}

Although the similarities with atherosclerosis were recognised as long ago as 1917 [Libman 1917], they were largely disregarded until recently [Roberts 1986; Mohler 2000; Demer 2001]. The histological studies described above have highlighted the common features but also confirmed differences in the cellular and mineral components of the two lesions.

Smooth muscle proliferation and lipid-laden macrophages (or foam cells) are prominent features of vascular atheroma but are virtually absent in stenotic aortic valves. In addition, mineralisation is an earlier and more extensive feature of aortic valve lesions compared with atherosclerosis [Otto et al 1994]. These differences may, in part, explain why only $40 \%$ of patients with severe aortic stenosis have significant coronary artery disease [Lombard and Selzer 1987; Vandeplas et al 1988; Mautner and Roberts 1992; Rapp et al 2001; Peltier et al 2003] and why the majority of patients with coronary artery disease do not have aortic stenosis. As the underlying pathology for the two conditions appears to be similar, it is likely that 
other unknown factors influence the development of valvular as opposed to vascular lesions [Otto and O’Brien 2001].

\subsection{CLINICAL PRESENTATION}

Patients present with either an incidentally noted asymptomatic systolic murmur or with symptoms of severe disease including angina, exertional syncope, breathlessness, and reduced exercise tolerance or lethargy. In simple terms, progressive obstruction to outflow results in a gradual rise in left ventricular pressures, left ventricular hypertrophy, and diastolic dysfunction. Once the degree of stenosis is severe, further small decreases in aortic valve area (AVA) result in large changes in the pressure gradient across the valve. Symptoms and decompensation arise due to the development of inadequate cardiac reserve, myocardial oxygen demand mismatch or pressure overload of the left ventricle. Symptoms rarely occur unless the degree of stenosis is of at least moderate severity (with an AVA of less than $1.0 \mathrm{~cm}^{2}$ ) but patients may remain asymptomatic for long periods with even very severe stenosis [Lombard and Selzer 1987].

\subsubsection{ClliniCAL RISK FACTORS}

In keeping with the apparent parallels with atherosclerosis, calcific aortic stenosis is associated with coronary artery disease [Mautner and Roberts 1992; Peltier et al 2003] and many of its risk factors (Table 1.1) [Stewart et al 1997]. Calcific aortic stenosis is also seen in association with severe homozygous familial hypercholesterolaemia, and its development appears to be influenced by the length of 
exposure to elevated serum cholesterol concentrations [Rallidis et al 1998]. Interestingly, aggressive lipid-lowering therapy with plasmapheresis has been reported to regress aortic stenosis in such patients [Keller et al 1986]. Milder forms of hypercholesterolaemia have also been associated with calcific aortic stenosis [Aronow et al 1987; Wilmshurst et al 1997; Chui et al 1999], particularly in patients with non-rheumatic tricuspid valves [Chui et al 1999].

TABLE 1.1 Risk factors for calcific aortic stenosis

\section{Clinical}

Age

Male sex

Smoking

Hypertension

Diabetes mellitus

Coronary artery disease

Chronic renal failure

Paget's disease

Hyperparathyroidism

\section{Biochemical}

Hyperlipidaemia (LDL and Lp (a))

Hypercalcaemia

Elevated serum creatinine

LDL - Low-density lipoprotein; Lp (a) - Lipoprotein a.

Conditions affecting calcium metabolism, such as chronic renal impairment with secondary hyperparathyroidsim [Maher et al 1987; Straumann et al 1992; Umana et al 2003] and advanced Paget's disease [Hultgren 1998], predispose individuals to aortic valve calcification and accelerated stenosis. Such patients also tend to have 
diffuse cardiac calcification affecting the mitral valve, myocardium and conducting system.

A number of twin studies and case reports suggest that hereditary factors may influence the development of calcific aortic valve stenosis [Lewis and Henderson 1990; Tentolouris et al 1993]. There has been a single report of a genetic association between aortic stenosis and a vitamin D receptor polymorphism [Ortlepp et al 2001] but this finding has yet to be confirmed.

\subsection{INVESTIGATIONS}

The assessment of valvular stenosis and monitoring of disease progression has only been possible over the last five decades using cardiac catheterisation, echocardiography and more recently magnetic resonance (MR) imaging and computed tomography (CT). Magnetic resonance may have some advantages over echocardiography in assessment of stenosis severity [Didier et al 2000], but its availability is limited and measurements are time consuming to perform. Although currently limited to clinical research, electron beam CT has recently been validated as a means of quantifying aortic valve calcification [MacMillan et al 1988; Lippert et al 1995; Kizer et al 2001]. However, echocardiography remains the current gold standard for monitoring of disease progression and left ventricular function in patients with aortic stenosis. 
The severity of aortic valve stenosis is assessed using both two-dimensional and Doppler echocardiography (Table 1.2). Narrowing of the aortic valve orifice results in acceleration of blood flow across the valve. Using spectral Doppler, the velocity of blood passing through the left ventricular outflow tract (pre-valve) and aortic valve orifice (post-valve) can be measured and is usually expressed in metres per second $(\mathrm{m} / \mathrm{s})$. The peak instantaneous pressure gradient across the aortic valve has a simple relationship with the peak post-valve velocity and is described as four times the square of the velocity (modified Bernoulli equation). For example, a peak post-valve velocity of $4 \mathrm{~m} / \mathrm{s}$ gives an instantaneous pressure gradient of $4 \times 4^{2}=64$ millimetres of mercury $(\mathrm{mmHg})$. Where there are concerns that impaired left ventricular function limits the ability to generate an adequate pressure gradient across the valve, measurement of the AVA may need to be made using direct planimetry or indirectly using the continuity equation. On occasions, dobutamine stress echocardiography may be used as a method of distinguishing true aortic stenosis causing left ventricular dysfunction from aortic pseudostenosis where the impairment of the left ventricle causes poor excursion of the aortic valve cusps giving the impression of stenotic valvular restriction. 


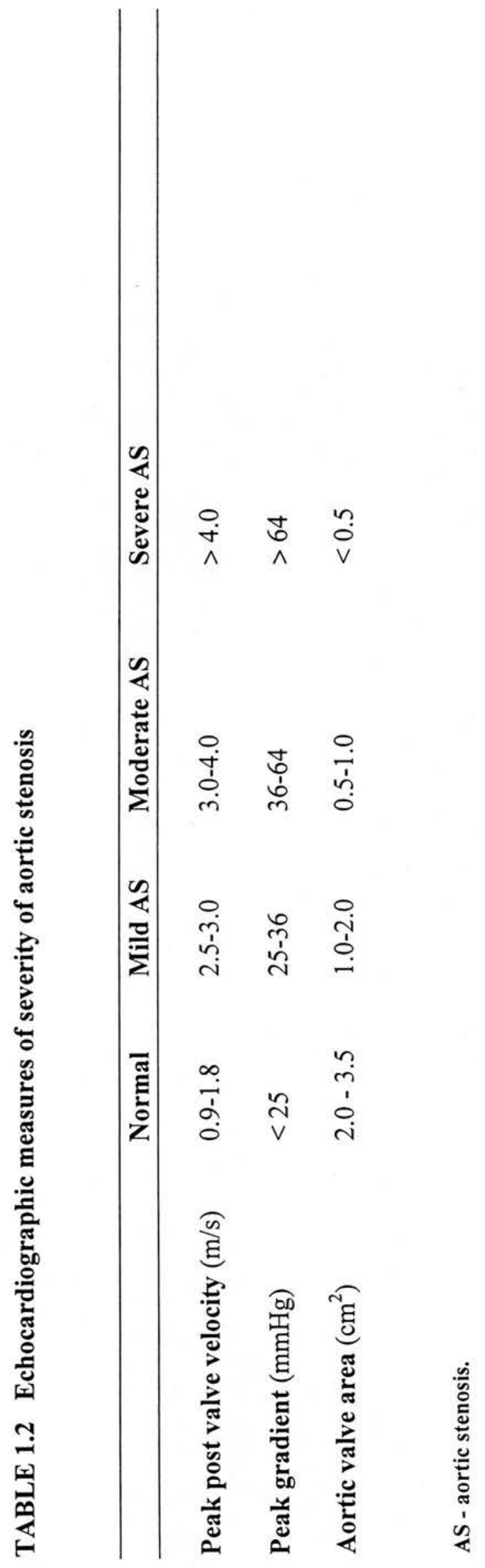




\subsubsection{DISEASE PROGRESSION}

Echocardiography provides the most accurate evaluation of disease progression, which can be unpredictable and extremely variable. Some individuals show little or no evidence of deterioration over time, yet others progress rapidly from mild to severe stenosis within a few years.

In patients with aortic valve sclerosis, progression to stenosis (arbitrarily defined as a peak post-valve velocity $\geq 2.5 \mathrm{~m} / \mathrm{s}$, or peak gradient $\geq 25 \mathrm{mmHg}$ ) is a relatively slow process with mean increases in peak post-valve velocity and peak gradient of $0.07 \mathrm{~m} / \mathrm{s}$ and $1.4 \mathrm{mmHg}$ per year respectively [Faggiano et al 2003]. However, once the valve is classified as stenotic, disease progression is more rapid with average increases of $0.3 \mathrm{~m} / \mathrm{s}$ and $7-8 \mathrm{mmHg}$ per year, corresponding to a decrease in AVA of $0.1 \mathrm{~cm}^{2}$ per year [Roger et al 1990; Faggiano et al 1992; Peter et al 1993; Brener et al 1995; Otto et al 1997].

\subsubsection{Predictors of Disease PROgRession and Clinical outCome}

Disease progression and clinical outcome have been linked to many of the risk factors for calcific aortic stenosis including age, male sex, hyperlipidaemia, hypertension, diabetes mellitus, smoking, hypercalcaemia and chronic renal impairment [Peter et al 1993; Bahler et al 1999; Palta et al 2000; Ngo et al 2001; Wongpraparut et al 2002]. However, much of the evidence is conflicting and limited by the retrospective nature of the studies. The most consistent and strongest predictors of disease progression are severity of stenosis at baseline [Otto et al 1997; Bahler et al 1999] and degree of valvular calcification [Davies et al 1991; 
Bahler et al 1999]. The more severe the stenosis at baseline and the more heavily calcified the valve, the faster the rate of progression. Clinical outcome is also influenced by the degree of valvular calcification, with nearly $80 \%$ of patients with moderate to severe calcification who progress rapidly $(>0.3 \mathrm{~m} / \mathrm{s} / \mathrm{yr})$ either dying or undergoing AVR within 2 years [Rosenhek et al 2000].

\subsection{MANAGEMENT OF CALCIFIC AORTIC STENOSIS}

At the present time, there is no known therapy that can slow or reverse disease progression in patients with calcific aortic stenosis. Current management includes monitoring disease progression, and ensuring patient awareness of the need for antibiotic prophylaxis against infective endocarditis. For those patients with severe symptomatic disease, the therapeutic options include conventional medical therapy for symptom control and AVR.

\subsubsection{GeNERAL ADVICE}

All patients should be advised of the need for antibiotic prophylaxis against endocarditis for dental and other invasive procedures. Patients with moderate or severe disease should be advised to avoid strenuous physical exercise and competitive sport because of the risk of sudden death, and to report promptly the onset of symptoms. 


\subsubsection{MONITORING OF DISEASE PROGRESSION}

Since disease progression is so unpredictable, the majority of patients should be reviewed regularly to monitor changes in stenosis severity and watch for the onset of symptoms. As a rule of thumb, asymptomatic patients with mild to moderate stenosis require review and echocardiography every 1-2 years, and those with moderate to severe stenosis every 6-12 months. Patients developing symptoms between appointments should be reviewed immediately.

\subsubsection{ASYMPTOMATIC SEVERE AORTIC STENOSIS}

One contentious area of management is determining the optimal timing for AVR. It is universally accepted that surgery is indicated as soon as symptoms appear in patients with severe stenosis. Although many cardiologists are loath to refer patients without symptoms for valve surgery, there are some who feel uncomfortable managing patients with severe asymptomatic valvular stenosis because of the potential risk for sudden cardiac death. However, this is rare and occurs in less than $1 \%$ of asymptomatic patients per year [Bonow et al 1998]. The combined risk of AVR (2-10\% mortality) and prosthesis-related complications $(2-3 \% / y r)$ is thus greater than the risk of sudden cardiac death. "Watchful waiting" is therefore recommended.

The onset of symptoms in patients with severe stenosis may be subtle and insidious, particularly in the elderly where co-morbidity may mislead or obscure the presentation. For this reason careful history taking for changes in exercise tolerance 
as well as the classical symptoms of breathlessness, chest pain and syncope is required. In cases where patients may be underplaying symptoms, attributing them to "old age", or unknowingly avoiding activity that induces symptoms, physician supervised exercise testing may be helpful in both revealing symptoms as well as determining the haemodynamic response to exercise. Patients who develop symptoms during exercise, become hypotensive, manifest marked ST segment changes or develop ventricular arrhythmias are at high risk and should be considered for valve replacement [Chambers 1999; Amato et al 2001; Carabello 2002].

\subsubsection{SYMPTOMATIC SEVERE AORTIC STENOSIS}

As soon as patients with severe aortic stenosis develop symptoms the treatment of choice is AVR because this substantially improves the quality of life and prognosis. In those patients declining valve surgery, or the frail elderly in whom major cardiac surgery would be inappropriate, palliation with conventional medical therapy, or in exceptional circumstances, balloon valvotomy are the only alternatives. Percutaneous AVR is a promising new technique that is currently under development in highly selected patient populations [Boudjemline and Bonhoeffer 2002; Cribier et al 2002].

\subsubsection{MEDICAL THERAPY}

Breathlessness. Patients with evidence of pulmonary congestion may benefit from the judicious use of diuretics, vasodilators and positive ionotropic agents such as digoxin. Excessive use of diuretics should be avoided since patients with severe aortic stenosis often have diastolic dysfunction and depend on an adequate pre-load in order to maintain their cardiac output. 
Despite the widespread belief that angiotensin-converting enzyme (ACE) inhibitors can cause dangerous hypotension in severe aortic stenosis, and are therefore contraindicated, there are little data to support this. From the limited literature available, two small studies demonstrated that first dose hypotension did not occur in patients with severe aortic stenosis, and that cardiac output and symptoms improved substantially [Grace et al 1991; Martinez-Sanchez et al 1996]. Although further study is required, some patients with heart failure and severe aortic stenosis could benefit from ACE inhibitors provided that they are carefully introduced in a hospital setting. Certainly those patients already established on therapy need not have it withdrawn since this may precipitate the onset of heart failure.

Digoxin can be helpful in the management of heart failure but should only be used in the presence of atrial fibrillation or where there is documented evidence of left ventricular systolic dysfunction. Atrial fibrillation is not well tolerated in the presence of severe stenosis and restoration to sinus rhythm (through electrical cardioversion or pharmacological cardioversion using amiodarone) should be attempted wherever possible.

Angina. In those individuals where angina is the predominant symptom, cautious use of beta-blockers and nitrates may be of benefit. Where coronary heart disease is suspected secondary prevention would be appropriate. 
Syncope. Patients with syncope or pre-syncope should be further evaluated with a 24-hour cardiac monitor since aortic stenosis is commonly associated with atrioventricular block. There is no specific therapy for syncope unless it is caused by a bradyarrhythmia or tachyarrhythmia, where pacemaker insertion or antiarrhythmic therapy respectively should be considered.

\subsubsection{BALLOON VALVOTOMY}

Although balloon valvotomy plays an important role in the management of adolescents and young adults with aortic stenosis, it has largely been abandoned in older patients. The functional improvement obtained is limited, the re-stenosis and complication rates high, and the long-term outlook poor $(<80 \%$ survival at 1 year $)$ [Robicsek et al 1988; Bonow et al 1998]. On rare occasions, balloon valvotomy may play a role in patients with a limited life expectancy for other reasons, or as a bridge to AVR in critically ill patients with cardiogenic shock.

\subsubsection{AORTIC VALVE REPLACEMENT}

Aortic valve replacement incurs the virtual abolition of symptoms associated with improvements in physical functioning and quality of life, and a dramatic improvement in survival. Operative mortality in middle-aged adults is in the region of 5-8\% [Linblom et al 1990; Sprigings and Forfar 1995; Kvidal et al 2000]; 5- and 10-year survival rates after aortic valve replacement are approximately $80 \%$ [Galloway et al 1990; Linblom et al 2000] and 65\% respectively [Linblom et al 1990], and are similar to actuarial survival rates for the general population [Linblom et al 1990]. 
Factors associated with a higher operative mortality include increasing age [Sharony et al 2003], the presence of renal impairment, cerebrovascular and peripheral vascular disease [Gilbert et al 1999], the presence of impaired left ventricular function [Sharony et al 2003], and the need for simultaneous coronary artery bypass grafting [Sprigings and Forfar 1995]. Despite the increased operative risk associated with the presence of left ventricular failure, this is not an absolute contraindication to surgery. Indeed these patients may have most to gain from valve surgery in terms of improvements in prognosis.

\subsubsection{AORTIC VALVE REPLACEMENT IN OCTOGENARIANS}

Successful AVR is becoming increasingly common in patients over the age of eighty. Despite evidence suggesting that it should be offered to all suitable patients regardless of age, several studies have demonstrated a reluctance to refer older patients for valve surgery [Lindroos et al 1993; Abdul-Hamid and Mulley 1999; Bouma et al 1999]. This probably reflects both patient and physician misconceptions of the risks and benefits of operative intervention.

Although operative mortality is higher in octogenarians (nearer 5-15\%), these individuals have almost as much to gain as their younger counterparts in terms of improved prognosis (5-year survival being 55-70\%). Of perhaps greater importance is that the majority of survivors achieve a significant reduction in symptoms [Olsson et al 1992; Olsson et al 1996; Gilbert et al 1999; Sundt et al 2000; Kohl et al 2001] associated with a marked improvement in physical functioning and quality of life 
[Olsson et al 1992; Sprigings and Forfar 1995; Olsson et al 1996; Sundt et al 2000]. Although intensive care [Gilbert et al 1999; Sundt et al 2000] and overall hospital stay [Olsson et al 1992; Sundt et al 2000; Kohl et al 2001] may be longer, the majority return to their own homes and retain their independence on discharge [Olsson et al 1992; Gilbert et al 1999]. However, post-operative complications are more common with a higher incidence particularly of stroke (4\%) and acute renal failure (7-10\%) [Sundt et al 2000]. In contrast with younger patients, octogenarians are usually offered a bioprosthetic (as opposed to a mechanical) valve, thus reducing the risk of valve thrombosis and anticoagulant associated haemorrhage.

\subsubsection{POTENTIAL ROLE FOR HMG COA REDUCTASE INHIBITORS}

Hydroxymethylglutaryl coenzyme A (HMG CoA) reductase inhibitors or statins are now well established in the primary and secondary prevention of coronary artery disease [Shepherd et al 1995; The Heart Protection Study Collaborative Group 2002]. Several studies have also shown that these drugs can cause regression of coronary artery disease [Zhao et al 1993] as well as reduce the calcific volume of coronary plaques [Callister et al 1998]. Given the clinical association of calcific aortic stenosis with hyperlipidaemia and coronary artery disease, and the striking histological similarities with atheroma, the speculation that statins may have the potential to influence disease progression in aortic stenosis is an intriguing hypothesis [Mohler 2000; Pearlman 2002].

Recent retrospective studies [Aronow et al 2001; Novaro et al 2001; Pohle et al 2001; Bellamy et al 2002; Shavelle et al 2002] have demonstrated that statins may 
delay disease progression in aortic stenosis through their lipid-lowering and antiinflammatory actions [Bellamy et al 2002]. These observational data should be interpreted with caution since none of these studies were randomised, and the statin doses were small. This preliminary evidence has been the rationale for establishing a randomised controlled trial of statin therapy in patients with aortic stenosis.

\subsection{CONCLUSION}

The need for an alternative to aortic valve surgery is highlighted by the increasing longevity of the population and rising prevalence of aortic stenosis. New therapeutic strategies to limit disease progression are needed in order to delay, and potentially avoid, the need for valve surgery.

\subsection{AIMS AND HYPOTHESES}

The aims of this thesis are to evaluate new methods of assessing and monitoring aortic stenosis, to clarify the factors that influence progression and outcome of aortic stenosis, and identify novel therapies for the disease. At the same time I sought to confirm the retrospective data suggesting that lipid-lowering therapy reduces coronary artery calcification.

The specific aims of the thesis were:

\section{Chapter 3}

In patients with calcific aortic stenosis; 
1. to establish the validity and reproducibility of helical CT in the quantification of aortic valve calcium (AVC) burden.

2. to establish the reproducibility of echocardiographic measures of aortic stenosis severity.

3. to determine if there was a relationship between the severity of aortic valve stenosis determined by echocardiography and the degree of valvular calcification quantified by helical CT.

Hypothesis;

In patients with calcific aortic stenosis, aortic jet velocity, determined by echocardiography, correlates with the AVC score measured by helical CT.

\section{Chapter 4}

4. To conduct a randomised double-blind placebo-controlled trial of high dose lipid-lowering therapy in patients with calcific aortic stenosis in order to determine its effect on the progression of aortic stenosis and aortic valve calcification.

Hypothesis;

In patients with calcific aortic stenosis lipid-lowering therapy will halt the progression, or induce regression, of the valvular disease process. In particular, lipidlowering therapy will:

(i) reduce the peak aortic jet velocity.

(ii) reduce the calcific volume of the aortic valve. 


\section{Chapter 5}

5. To prospectively determine the effect of intensive lipid-lowering therapy on the progression of coronary artery calcification in patients with calcific aortic stenosis.

Hypothesis;

In patients with calcific aortic stenosis lipid-lowering therapy delays progression of coronary artery calcification.

\section{Chapter 6}

6. To identify prospectively traditional clinical risk factors and other novel predictors of cardiovascular risk, including inflammatory, vascular and cardiac markers that are associated with disease progression and clinical outcome in patients with calcific aortic stenosis.

Hypothesis;

In patients with aortic stenosis cardiovascular risk factors predict disease progression and clinical outcome. 


\section{CHAPTER 2}

MATERIALS AND METHODS 


\subsection{INTRODUCTION}

In this thesis, I have explored the interrelationship of atherosclerotic vascular disease and calcific aortic stenosis. This required a comprehensive assessment of valvular, myocardial and vascular variables. I have therefore measured valvular, myocardial and vascular structure and function in patients with calcific aortic stenosis. This has been complemented by the measurement of biochemical markers of systemic inflammation, and myocardial disease as well as non-invasive measures of vascular function such as arterial stiffness.

Echocardiography and CT are complementary methods of assessing the severity of aortic valve disease and its progression. Computed tomography also allows the measurement of coronary artery calcium, a marker of coronary artery disease, and pulse wave analysis provides a measure of arterial stiffness, a marker of peripheral arterial disease. These modalities have been used as the mainstay for the assessment of recruited patients.

\subsection{THE SALTIRE TRIAL}

The Scottish Aortic stenosis and Lipid lowering Therapy Impact on REgression (SALTIRE) trial was a prospective, randomised, double-blind placebo-controlled trial designed to assess the influence of high dose statin therapy on disease progression in patients with calcific aortic stenosis. It was funded by a project grant from the British Heart Foundation (PG/2000/044) and also supported by an 
unrestricted educational grant award from Pfizer UK, and by the Wellcome Trust Clinical Research Facility in Edinburgh.

\subsubsection{DEFINITION OF STUDY POPULATION}

Patients aged over 18 years with calcific aortic stenosis, an aortic jet velocity of at least $2.5 \mathrm{~m} / \mathrm{s}$, and aortic valve calcification on echocardiography were eligible for inclusion in the SALTIRE trial.

Exclusion criteria were child-bearing potential without contraception, active or chronic liver disease, a history of alcohol or drug abuse, severe mitral valve stenosis (mitral valve area, $<1 \mathrm{~cm}^{2}$ ), severe mitral or aortic regurgitation [Zoghbi et al 2003], significant left ventricular dysfunction (ejection fraction $<35 \%$ ), planned AVR, intolerance of statins, current statin therapy or a potential benefit from statin therapy (according to the treating physician), a baseline serum total cholesterol concentration of $<4.0 \mathrm{mmol} / \mathrm{L}$, and presence of a permanent pacemaker or cardiodefibrillator.

Of the patients screened, 455 were eligible for inclusion, 173 agreed to participate, and 155 ultimately underwent randomisation.

\subsubsection{STUDY PROTOCOL AND CLINICAL FOLLOW-UP}

Between March 2001 and April 2002, the blinded study co-ordinator randomly assigned eligible patients by the minimisation technique [Treasure and MacRae 1998] with the use of a dedicated, locked computer program (Edinburgh University) incorporating the following eight variables: age, sex, smoking habit, hypertension, 
diabetes mellitus, serum cholesterol concentration, aortic jet velocity, and aortic valve calcium score. Patients were assigned to either $80 \mathrm{mg}$ of atorvastatin (Lipitor, Pfizer) or matched placebo as a single daily dose. Numbered containers were used.

Patients were assessed at baseline, at 2 and 6 months, and 6 monthly thereafter for a minimum of 2 years. The following clinical end-points were recorded throughout the study; cardiovascular and all cause mortality, AVR (whether for severe symptomatic stenosis or not), the development of symptoms attributable to severe aortic stenosis (confirmed by the patient's treating physician), and hospitalisation (both all cause and cardiovascular causes). Functional status and adverse events were recorded at each visit. Echocardiography and CT were performed annually.

\subsection{BIOCHEMICAL VARIABLES}

Fasting venous blood samples were taken annually. Samples for serum electrolytes, lipid profile and calcium concentrations were sent to the regional clinical laboratory for immediate analysis. Samples for estimation of serum brain natriuretic peptide (BNP) and C-reactive protein (CRP) concentrations were centrifuged at $4^{\circ} \mathrm{C}$ and stored at $-80^{\circ} \mathrm{C}$ for later analysis.

\subsubsection{INFLAMMATORY MARKERS}

Serum CRP concentration is a sensitive but non-specific marker of systemic inflammation that is elevated in patients with vascular disease [Van der Meer et al 2002] and calcific aortic stenosis [Galante et al 2001]. It predicts clinical outcome in 
patients with coronary heart disease and in apparently healthy subjects [Ridker et al 1998]. Plasma CRP concentrations were measured using a highly sensitive nephelometric assay with a monoclonal antibody to CRP coated on polystyrene beads (Dade Behring UK Ltd). The sensitivity of the assay was $0.175 \mathrm{mg} / \mathrm{L}$, with intra-assay and inter-assay coefficients of variation of less than 5\% [Chenillot et al 2000; Jialal et al 2001].

\subsubsection{ENDOGENOUS CARDIAC HORMONES}

Natriuretic hormones are endogenous cardiac hormones that are secreted from the cardiac atria and ventricles. Brain natriuretic peptide and its aminoterminal portion $\mathrm{N}$-terminal BNP, are released in response to increased wall stretch and effect both natriuresis and diuresis with a resultant decrease in intravascular volume, blood pressure and pre-load. Serum concentrations are elevated in left ventricular dysfunction, and correlate with New York Heart Association (NYHA) class and prognosis [Tsutamoto et al 1997; McDonagh et al 2001; Berger et al 2002; Lubien et al 2002]. Serum BNP concentrations are also elevated in other structural heart disease [Nakamura et al 2002] including left ventricular hypertrophy and aortic stenosis. The $\mathrm{N}$-terminal peptides are inactive and more stable [Boomsma et al 2001], and therefore N-terminal pro-BNP was measured using a chemiluminescent immunoassay (Roche Diagnostics Ltd, Lewes, UK) on an Elecsys 2010 analyser. The sensitivity of the assay was $5 \mathrm{pg} / \mathrm{mL}$, with intra-assay and inter-assay coefficients of variation of $<5 \%$ [Hartman et al 2004]. 


\subsection{ECHOCARDIOGRAPHY}

Echocardiography, or cardiac ultrasound, relies on the processing of high frequency sound waves emitted from and received by an ultrasound transducer using a frequency of $2-5-\mathrm{MHz}$. It is a non-invasive and relatively quick technique to determine cardiac structure and function. There are three main modes of operation: M-mode (single-dimensional), two-dimensional, and Doppler (spectral Doppler and colour flow mapping). Spectral Doppler includes pulsed and continuous wave Doppler, which are used to assess velocity of blood flow and cardiac tissue at a given specific point. Colour flow mapping is superimposed on either one or twodimensional images to provide a picture of blood flow using different colours for opposing directions of flow.

Echocardiography is the gold standard modality for the assessment of stenosis in patients with aortic valve disease and was the principal modality employed in this thesis.

\subsubsection{SCANNING PROTOCOL AND TECHNIQUE}

A single dedicated research sonographer, blinded to treatment allocation and CT results, performed all echocardiographic examinations and analyses. The echocardiograms were all performed on either an ATL-3000 (Philips Medical Systems (UK) Ltd, Stevenage, UK), or a Vingmed System 5 Performance (BMS (Scotland) Ltd, Belshill, UK) cardiac ultrasound machine using a 3-MHz transducer. Each patient was always scanned using the same equipment. All measurements were determined online, averaged from three cardiac cycles (five cycles if the patient was 
in atrial fibrillation), and recorded onto super-VHS videotape and optical disk according to a standard protocol.

Aortic valve calcification was graded using the Rosenhek classification [Rosenhek et al 2000]. In the presence of calcification bicuspid valves are very difficult to diagnose using echocardiography, and diagnosis of a bicuspid valve was therefore only made if the appearance was unequivocal. In uncertain cases the valve was classified as tricuspid. Severity of aortic valve stenosis was determined using the peak aortic jet velocity, peak and mean aortic gradient, and AVA. Peak and mean aortic valve pressure gradients were calculated using the modified Bernoulli equation. Aortic valve area measured by the continuity equation requires measurement of left ventricular outflow tract velocity, peak aortic jet velocity and left ventricular outflow tract diameter.

Left ventricular outflow tract velocity was measured from an apical approach using pulsed Doppler just proximal to the aortic valve leaflets. Peak aortic jet velocity was determined using continuous wave Doppler from three sites; the apical long axis approach, the right upper sternal border, and the suprasternal notch using the stand alone probe, and the window generating the highest signal was recorded. Left ventricular outflow tract diameter (LVOTD) was measured in the parasternal long axis view in mid systole just below the aortic valve. It was measured at baseline and maintained constant throughout the study, given that LVOTD remains static over time and variation in the measurement of this dimension results in the greatest variability in valve area assessment [Myreng et al 1990]. 
The above measures of aortic stenosis severity have all been well validated when compared with invasive data, and as predictors of clinical outcome. Peak aortic jet velocity is the most reproducible measure, and is the strongest predictor of clinical outcome [Otto 2006].

\subsection{COMPUTED TOMOGRAPHY}

Computed tomography allows the generation of anatomical soft tissue images in the axial plane providing complementary data to echocardiography. Prior to the development of electron beam and helical CT, conventional CT was limited by slow scan times, and was therefore unsuitable for the assessment of rapidly moving cardiac structures. Helical CT, which only become widely available 5 years ago, has a gantry capable of continuous rotation and very rapid image acquisition. Technology has advanced dramatically since, such that with the introduction of multi-slice technology and ECG gating it is now possible to produce images virtually free of motion artefact. At the inception of the SALTIRE trial helical CT was the most advanced technology available.

Cardiac CT was initially introduced to look for the presence of coronary artery calcification as evidence of coronary atheroma, and subsequently to monitor disease progression in patients with coronary artery disease. Disease severity determined by invasive coronary angiography correlates well with coronary calcium burden assessed by both electron beam [Haberl et al 2001] and helical [Achenbach et al 
2001; Nieman et al 2001] CT. In the late 1990's there was limited retrospective evidence that progression of coronary artery calcification may be delayed by the use of statin therapy [Callister et al 1998].

Aortic valve calcification is a common incidental finding on cardiac CT and although not diagnostic of aortic valve stenosis, the more heavily calcified the valve is the more likely it is that stenosis will be present [Lippert et al 1995]. Electron beam CT has only recently been validated as a reproducible technique for the quantification of AVC [Kizer et al 2001], and prior to the commencement of the SALTIRE trial the validity of helical CT had not been determined. Validation of helical CT was therefore an integral part of the SALTIRE trial, as well as the assessment of the relationship between the severity of aortic valve stenosis and calcium burden (see Chapter 3).

\subsubsection{SCANNING PROTOCOL AND TECHNIQUE}

Computed tomography was performed using a double-helix scanner (Twin II Flash; Philips Medical Systems (UK) Ltd, Stevenage, UK). The region of the aortic valve and coronary arteries was assessed using $2.7 \mathrm{~mm}$ slices, with a pitch of 0.7 and an increment of $1.4 \mathrm{~mm}$ during held inspiration. Computed tomography scanner quality assurance was performed before each examination with calibration against a standard phantom. A single research radiographer performed all scans, and a single consultant radiologist performed all analyses, both being blinded to the echocardiography results. Off-line analysis of the cardiac images was conducted using an automated, computerised software program (Picker Cardiac Scoring). This employs a modified 
Agatston scoring method [Shemesh et al 1995] that uses a threshold of 90 Hounsfield Units (HU) to compensate for non-gated imaging. This modification produces comparable sensitivity and specificity to electron beam CT [Carr et al 2000]. Calcium scores were individually calculated for the aortic valve, and all three coronary arteries by summing the lesion scores for all sections containing calcium.

\subsection{PULSE WAVE ANALYSIS}

The link between blood pressure and cardiovascular disease has been clearly documented [Goldberg et al 1996]. Pulse wave analysis (PWA) by applanation tonography is a simple, non-invasive, reproducible method by which central aortic pressure waveforms can be determined from waveforms acquired peripherally at for example, the radial artery. The artery is compressed beneath the micromanometer probe tip, and arterial pressure is transmitted through the arterial wall to the sensor. Computer software calculates the central aortic pressure and waveform from the peripheral waveform using a generalised transfer function calibrated using peripheral blood pressure.

\subsubsection{SCANNING PROTOCOL AND TECHNIQUE}

Pulse wave analysis was performed at baseline and 6 monthly intervals thereafter. Patients were rested supine for 15 minutes prior to study. Radial PWA was performed using a high-fidelity applanation tonometer (Sphygmocor BPAS; PWV Medical, Sydney, Australia). After acquiring a series of consecutive waveforms, an averaged peripheral waveform was acquired and a generalised transfer function was 
used to generate a central aortic pressure waveform from which the pulse pressure, augmentation pressure and augmentation index were determined. Two measurements of augmentation within $5 \mathrm{mmHg}$ of each other were recorded on each occasion. Patients with atrial fibrillation were excluded, and recordings with poor quality waveforms were discarded: determined by visual inspection (SJC) with a minimum requirements in pulse height of $>100 \mathrm{mmHg}$, diastolic variation of $<5 \%$ and pulse height variation of $<5 \%$. Data on 105 patients were available at baseline, of these 20 patients had poor quality recordings and 9 patients were in atrial fibrillation, leaving a total of 84 patients for analysis.

\subsection{DATA ANALYSIS AND STATISTICS}

Computed tomography, BNP and CRP data were not normally distributed, and are expressed as median with interquartile ranges, or as mean with standard deviation (SD) after logarithmic transformation. Two-sided p values of less than 0.05 were considered to indicate statistical significance.

\subsubsection{ChaPTER 3}

\subsubsection{Reproducibility}

Reproducibility of echocardiographic and CT measures were determined by the method of Bland and Altman [Bland and Altman 1986] and expressed as the mean of the differences and the coefficient of repeatability (twice the standard deviation of the differences). As the difference of the two measures was proportional to their mean, the data for the AVC score underwent logarithmic transformation [Bland and Altman 1986]. Data were compared using regression analysis and analysis of 
variance (ANOVA) using StatView version 5.0.1 (SAS Institute Inc., Cary, NC, USA). Where ANOVA demonstrated significant differences in responses, post-hoc comparisons were made using Fisher's protected least squares difference (PLSD) test (StatView version 5.0.1).

\subsubsection{CHAPTER 4}

\subsubsection{Progression of aortic valve disease}

The two primary end-points were progression of stenosis, determined according to changes in aortic jet velocity on Doppler echocardiography, and progression of valvular calcification, measured by CT. Secondary end-points were a composite of clinical end-points (death from cardiovascular causes, AVR, or hospitalisation attributable to severe aortic stenosis), AVR, death from any cause, hospitalisation for any cause, and hospitalisation for cardiovascular causes.

On the basis of standard deviations of $0.32 \mathrm{~m} / \mathrm{s}$ per year [Faggiano et al 1992; Otto et al 1997] and 1100 arbitrary units (AU) per year [Shemesh et al 1995], we calculated that the planned sample size of 75 patients per group would give the study a power of $80 \%$ at a $5 \%$ significance level to detect a difference in the primary end-points of $0.15 \mathrm{~m} / \mathrm{s}$ per year in aortic jet velocity and $500 \mathrm{AU}$ per year in AVC score. These differences are equivalent to a reduction of more than $30 \%$ in the rate of progression of aortic stenosis. This would exclude a clinically significant effect in the majority of older patients with established disease, although a smaller effect may be clinically relevant in younger patients with mild aortic stenosis. 


\subsubsection{Interim analysis and data monitoring committee}

The data monitoring committee conducted two interim assessments of safety and an interim assessment of efficacy one year after enrolment began. The trial was to be terminated early in the event of a negative effect of treatment (i.e. $<0.05$ ) or a strong benefit of treatment (i.e. $\mathrm{p}<0.001$ ). On the recommendation of the data monitoring committee, the trial continued until the study was completed.

Analyses were performed using SPSS software, version 12.0, and SAS software, version $8 \mathrm{e}$. Intention-to-treat analyses were used for all clinical outcome variables. Disease progression was determined primarily by dividing the change between the baseline and final scans by the duration of follow-up. Treatment comparisons for the continuous outcome variables were based on an analysis of covariance, with the prerandomisation level of a variable used as a covariate. In a confirmatory analysis of the primary end-points, random coefficient models were fitted to incorporate all observations [Brown and Prescott 1999]. In the subgroup analyses, interaction terms between treatment and subgroup have been added to a model incorporating prerandomisation level, treatment, and subgroup to identify factors that were associated with a differential treatment effect within subgroups. Categorical variables have been analysed using Fisher's exact test. Two-tailed tests were used throughout.

\subsubsection{ChAPTER 5}

\subsubsection{Progression of coronary disease}

Coronary artery calcium scores are expressed in AU based on the $130 \mathrm{HU}$ threshold. The primary end-point, the rate of change of coronary calcium scores, was analysed 
with random coefficient models [Bland and Altman 1986; Cowell et al 2005], after logarithmic transormation of the scores. In summarising the data, we calculated the change in coronary artery calcium scores by dividing the change between the baseline and final scores by the duration of follow-up. Rate of change in coronary calcium score is expressed as percentage change per year or as absolute change in the logarithm of the coronary artery calcium score.

\subsubsection{CHAPTER 6}

\subsubsection{Predictors aortic stenosis progression and clinical outcome}

Disease progression was determined by dividing the change between the baseline and final scans by the duration of follow-up. Clinical end-points were; cardiovascular and all cause mortality, AVR (whether for severe symptomatic stenosis or not), and the development of symptoms attributable to severe aortic stenosis (confirmed by the patient's treating physician). To define predictors of outcome patients who reached a clinical end-point were compared with those who did not.

Analyses were performed using SPSS software, version 12.0, and SAS software, version $8 \mathrm{e}$. Predictors of progression were determined using regression analysis for continuous variables, and Chi-squared tests for categorical variables. Predictors of outcome were determined using Chi-squared tests for categorical variables, $t$-tests for continuous variables. 


\section{CHAPTER 3}

\section{AORTIC VALVE CALCIFICATION ON COMPUTED TOMOGRAPHY}

PREDICTS THE SEVERITY OF AORTIC STENOSIS

\section{Based on}

Cowell SJ, Newby DE, Burton J, White A, Northridge DB, Boon NA, Reid J. Aortic valve calcification on computed tomography predicts the severity of aortic stenosis. Clin Radiol 2003;58(9):712-6. 


\subsection{SUMMARY}

Background Disease progression in patients with aortic stenosis is more rapid in those with heavy calcification of the valve. The aims of this study were to determine the reproducibility of AVC scoring using helical $\mathrm{CT}$, and to compare the AVC scores with the severity of valve stenosis measured by echocardiography.

Methods One hundred and fifty-seven patients aged $68 \pm 11$ years (range 34-85) with aortic valve stenosis underwent dual array helical CT and Doppler echocardiography performed by independent blinded observers. The AVC score was determined using automated computer software calibrated with a phantom.

Results Doppler echocardiography demonstrated a post-valve velocity of $3.45 \pm 0.66 \mathrm{~m} / \mathrm{s}$ and a peak gradient of $49 \pm 11 \mathrm{mmHg}$. Computed tomography showed excellent reproducibility and the median score was 5858 (interquartile range 155514596) AU. The computed tomography AVC score positively correlated with the Doppler post-valve velocity and peak gradient $(\mathrm{r}=0.54, \mathrm{p}<0.0001$ for both) of the aortic valve. All patients with severe aortic stenosis had a calcium score of $>3700$ AU.

Conclusion Helical CT is a reproducible method of quantifying AVC burden. Calcification of the aortic valve is closely associated with the severity of aortic stenosis and heavy calcification suggests the presence of severe aortic stenosis that requires prompt cardiological assessment. 


\subsection{INTRODUCTION}

Calcific aortic stenosis is the commonest reason for valve replacement in the developed world. The condition may be due to progressive calcification of a congenitally bicuspid valve or 'degenerative' calcification of a morphologically normal valve [Pomerance 1972]. Irrespective of the aortic valve morphology, the histological features are surprisingly similar to those of coronary atheroma and include lipid deposition, fibrosis and calcification [Otto et al 1994].

Disease progression in aortic stenosis is variable and unpredictable, but appears to be most rapid in patients with valvular calcification [Davies et al 1991; Bahler et al 1999]. The presence of AVC more than doubles the annual increase in aortic valve gradient (9.7 versus $4.4 \mathrm{mmHg} / \mathrm{yr}$ ), and the echocardiographic grade of calcification correlates with the rate of disease progression [Davies et al 1991]. Indeed, moderate or severe aortic valve calcification is the strongest independent risk factor for an adverse clinical outcome with a 5-fold increase in the rate of death or AVR [Rosenhek et al 2000].

Computed tomography is being increasingly used as a non-invasive method of screening for atherosclerotic coronary artery disease [Breen et al 1992; Mautner et al 1994] with $80-100 \%$ sensitivity and $80 \%$ specificity [Harberl et al 2001]. There is an association between coronary artery disease and calcific aortic stenosis with approximately a third of patients with aortic stenosis having significant coronary stenoses on angiography [Mautner and Roberts 1992]. As a consequence, there have been several reports of 
incidental aortic valve calcification detected during CT examinations with a prevalence of 10-30\% [Woodring and West 1989; Lippert et al 1995]. However, there have been few reports [Lippert et al 1995] assessing the relationship between the degree of valvular calcification and the severity of aortic stenosis.

We wished to evaluate the reproducibility of helical CT in the quantification of AVC burden, and hypothesised that valvular calcification would correlate with the aortic postvalve velocity in patients with aortic stenosis.

\subsection{METHODS}

\subsubsection{SUBJECTS}

One hundred and fifty-seven patients being evaluated for the SALTIRE trial, participated in this substudy, which was undertaken with the approval of the local research ethics committee, in accordance with the Declaration of Helsinki, and with the written informed consent of each subject. Inclusion and exclusion criteria for the SALTIRE trial are outlined in Chapter 2. One patient, with a previous aortic root abscess, was excluded because of extensive aortic root calcification that obscured and prevented assessment of the aortic valve.

All patients underwent both echocardiography and CT within the month before randomisation to study therapy (atorvastatin $80 \mathrm{mg}$ daily or placebo). Only preintervention baseline data are presented here. 


\subsubsection{ECHOCARDIOGRAPHY}

The echocardiograms were performed on an ATL-3000 (Philips Medical Systems (UK) Ltd, Stevenage, UK) or a Vingmed System 5 Performance (BMS (Scotland) Ltd, Belshill, UK) cardiac ultrasound machine using a 3-MHz transducer for M-mode, and two-dimensional imaging with integral pulsed and continuous wave Doppler. The peak instantaneous aortic valve gradient was determined using the modified Bernoulli equation, and the AVA by the continuity equation. Aortic valve calcification was graded using the Rosenhek classification [Rosenhek et al 2000]. A single operator blinded to the results of the CT performed all echocardiographic examinations and analyses.

\subsubsection{COMPUTED TOMOGRAPHY}

The computed tomograms were performed using a dual array helical scanner (Twin II Flash; Philips Medical Systems (UK) Ltd, Stevenage, UK). The region of the aortic valve and coronary arteries was assessed using $2.7 \mathrm{~mm}$ slices, with a pitch of 0.7 and an increment of $1.4 \mathrm{~mm}$ during held inspiration. Operators blinded to the results of the echocardiogram performed all examinations and analyses. Computed tomography scanner quality assurance was performed prior to each examination with calibration against a standard phantom. Off-line analysis of the cardiac images was conducted using an automated, computerised software program (Picker Cardiac Scoring). This employs a modified Agatston scoring method [Shemesh et al 1995] that uses a threshold of $90 \mathrm{HU}$ to compensate for non-gated imaging. This modification produces comparable sensitivity and specificity to electron beam CT. Calcium scores were individually 
calculated for the aortic valve, and all three coronary arteries by summing the lesion scores for all sections containing calcium.

\subsubsection{REPRODUCIBILITY}

Two unselected random samples of 20 subjects each were taken from the study population. Subjects underwent repeated CT or echocardiography within 4 weeks of the first examination and before administration of the study medication.

\subsubsection{DATA ANALYSIS AND STATISTICS}

Data are expressed as mean $\pm \mathrm{SD}$. The calcium scores were not normally distributed and are expressed as median with interquartile ranges. The aortic valve and coronary artery calcium volume scores are expressed as AU). Reproducibility was assessed by the method of Bland and Altman [Bland and Altman 1986], and expressed as the mean of the differences and the coefficient of repeatability (twice the standard deviation of the differences). Since the difference of the two measures was proportional to their mean, the data for the aortic valve calcium score underwent logarithmic transformation [Bland and Altman 1986]. Data were compared using regression analysis and ANOVA using StatView v5.0.1 (SAS Institute Inc., Cary, North Carolina, USA). Where ANOVA demonstrated significant differences in responses, post-hoc comparisons were made using the Fisher's PLSD test (StatView v5.0.1). Statistical significance was taken at the $5 \%$ level. 


\subsection{RESULTS}

Subject characteristics are shown in Table 3.1. In keeping with the study population, subjects were predominantly male, elderly and had haemodynamically significant aortic stenosis. Both echocardiography and CT showed excellent reproducibility (Table 3.2).

All but two patients had significant aortic valve calcification on CT (Figure 3.1). The median AVC score was 5858 AU. The majority of patients (107/157) had detectable coronary artery calcification that predominantly affected the left anterior descending (LAD) coronary artery. There was no correlation between the magnitude of the aortic valve and total coronary calcium scores $(r=0.04, \mathrm{p}=0.61)$.

\subsubsection{COMPARISON BETWEEN ECHOCARDIOGRAPHY AND COMPUTED TOMOGRAPHY}

Echocardiographic grade of calcification correlated weakly with the computed tomography AVC score $(r=0.29, \mathrm{p}<0.001)$ and the peak aortic jet velocity $(r=0.40$, $\mathrm{p}<0.001)$. The aortic valve calcium score correlated strongly with the peak aortic jet velocity $(\mathrm{r}=0.54, \mathrm{p}<0.0001: \mathrm{y}=0.00004 \mathrm{x}+3.09$; Figure 3.2$)$ and the mean $(\mathrm{r}=0.54$, $\mathrm{p}<0.0001: \mathrm{y}=0.0008 \mathrm{x}+20.7)$ and peak gradient $(\mathrm{r}=0.54, \mathrm{p}<0.0001: \mathrm{y}=0.0013 \mathrm{x}+39.1)$ of the aortic valve, but only weakly correlated with the aortic valve area $(r=0.20, p=0.01)$. 
TABLE 3.1 Baseline characteristics

\section{Baseline subject characteristics}

Number

Age

Sex

Bicuspid Aortic

Valve

\section{Atrial Fibrillation}

Echocardiogram

Pre-valve velocity

Post-valve velocity

Peak gradient

Mean gradient

Valve area

Computed Tomogram*

Aortic Valve

$5858(1555-14596)$

$\mathrm{AU}$

Coronary Artery

LAD

Circumflex

Right

Total

Mean \pm SD.

* Median (interquartile range).

LAD - left anterior descending.
157

$68 \pm 11$

$71 \%$

years

male
5

11

$\begin{array}{rlr}1.08 & \pm 0.22 & \mathrm{~m} / \mathrm{s} \\ 3.45 & \pm 0.66 & \mathrm{~m} / \mathrm{s} \\ 49 & \pm 19 & \mathrm{mmHg} \\ 27 & \pm 11 & \mathrm{mmHg} \\ 1.02 & \pm 0.40 & \mathrm{~cm}^{2}\end{array}$




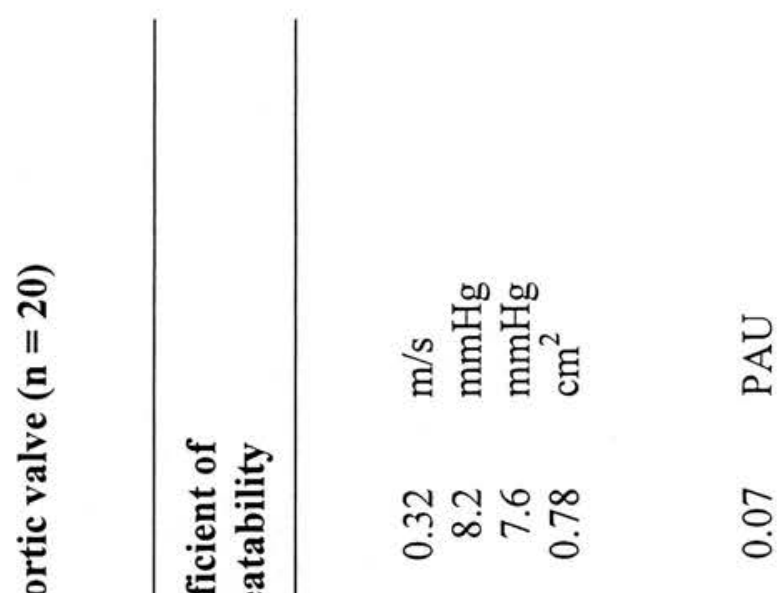

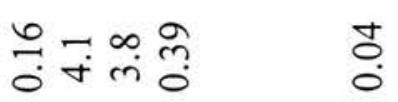

유유ํ융

亏

हั.

$\exists \circ \infty \stackrel{0}{\exists}$

$\mathrm{H}+\mathrm{H}+$

위든

:

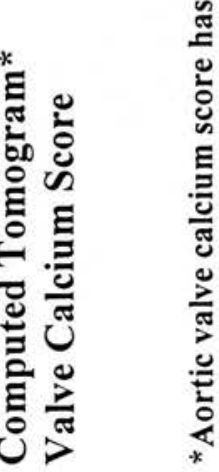




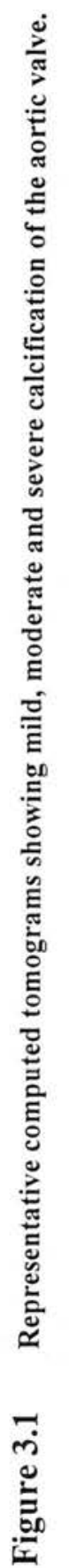

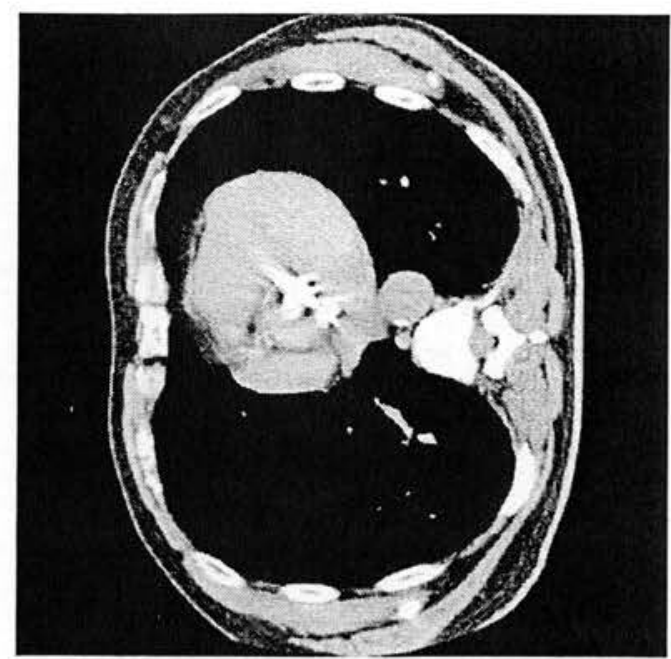

$\frac{1}{0}$

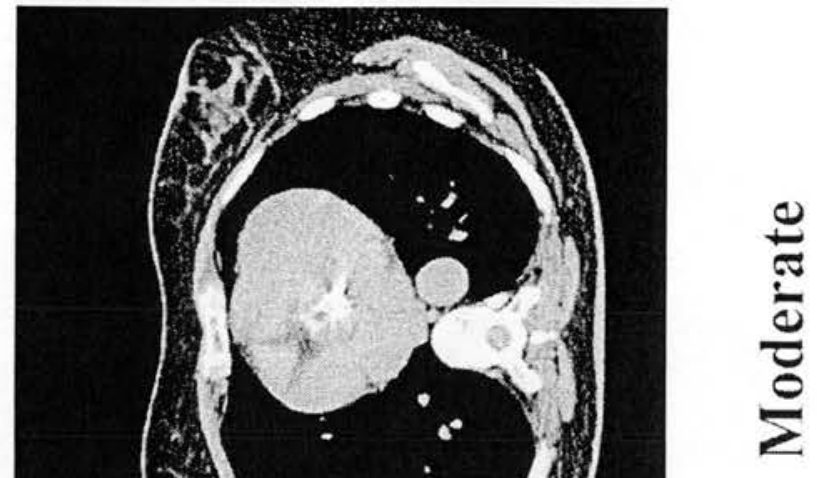

$\frac{0}{2}$

르

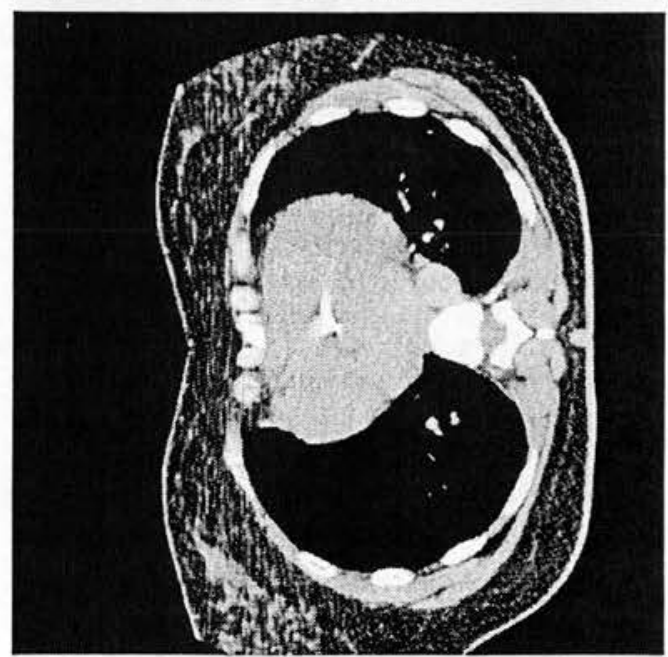


Figure 3.2 Correlation of aortic valve calcification and peak aortic jet velocity.

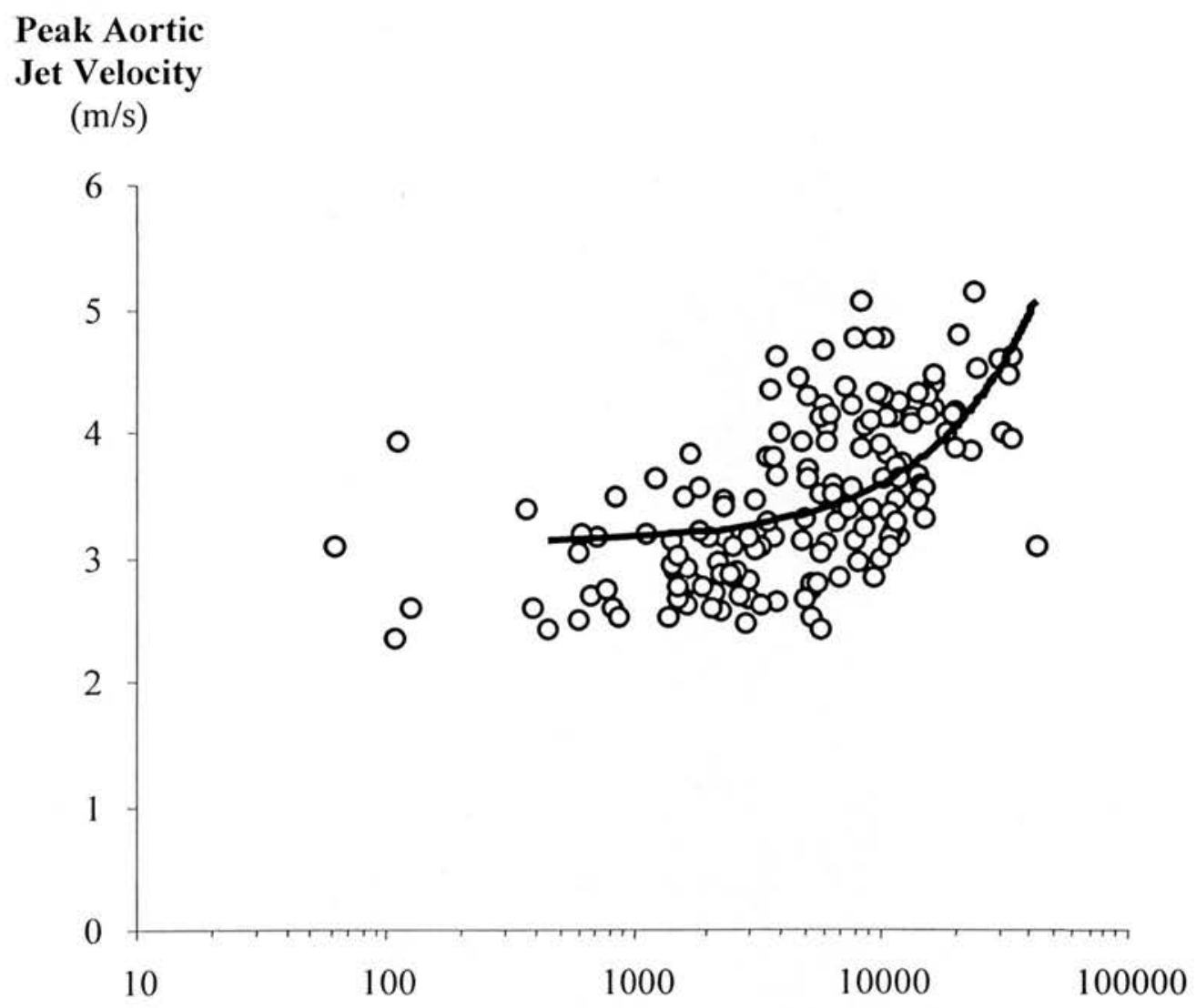

\section{Aortic Valve Calcium Score}

(HU)

$\mathrm{r}=0.54, \mathrm{p}<0.001$.

HU - Hounsfield Units. 
Stratifying the patients according to the quintiles of calcification demonstrated a progressive increase in the mean peak aortic jet velocity (Figure 3.3). All patients with severe aortic valve stenosis (peak aortic jet velocity $>4 \mathrm{~m} / \mathrm{s}$ ) had an AVC score of $>3700$ AU. This threshold gives a sensitivity of $100 \%$ and specificity of $50 \%$ that translates into a negative predictive value of $100 \%$ and a positive predictive value of $39 \%$ for the detection of severe aortic stenosis. A threshold of 6000 AU gives a sensitivity of $90 \%$ and specificity of $66 \%$ giving a negative predictive value of $95 \%$ and a positive predictive value of $45 \%$. 
Figure 3.3 Mean $( \pm$ SD) peak aortic jet velocity in the quintiles of aortic valve calcification.

\section{Peak Post-Aortic \\ ValveVelocity \\ $(\mathrm{m} / \mathrm{s})$}
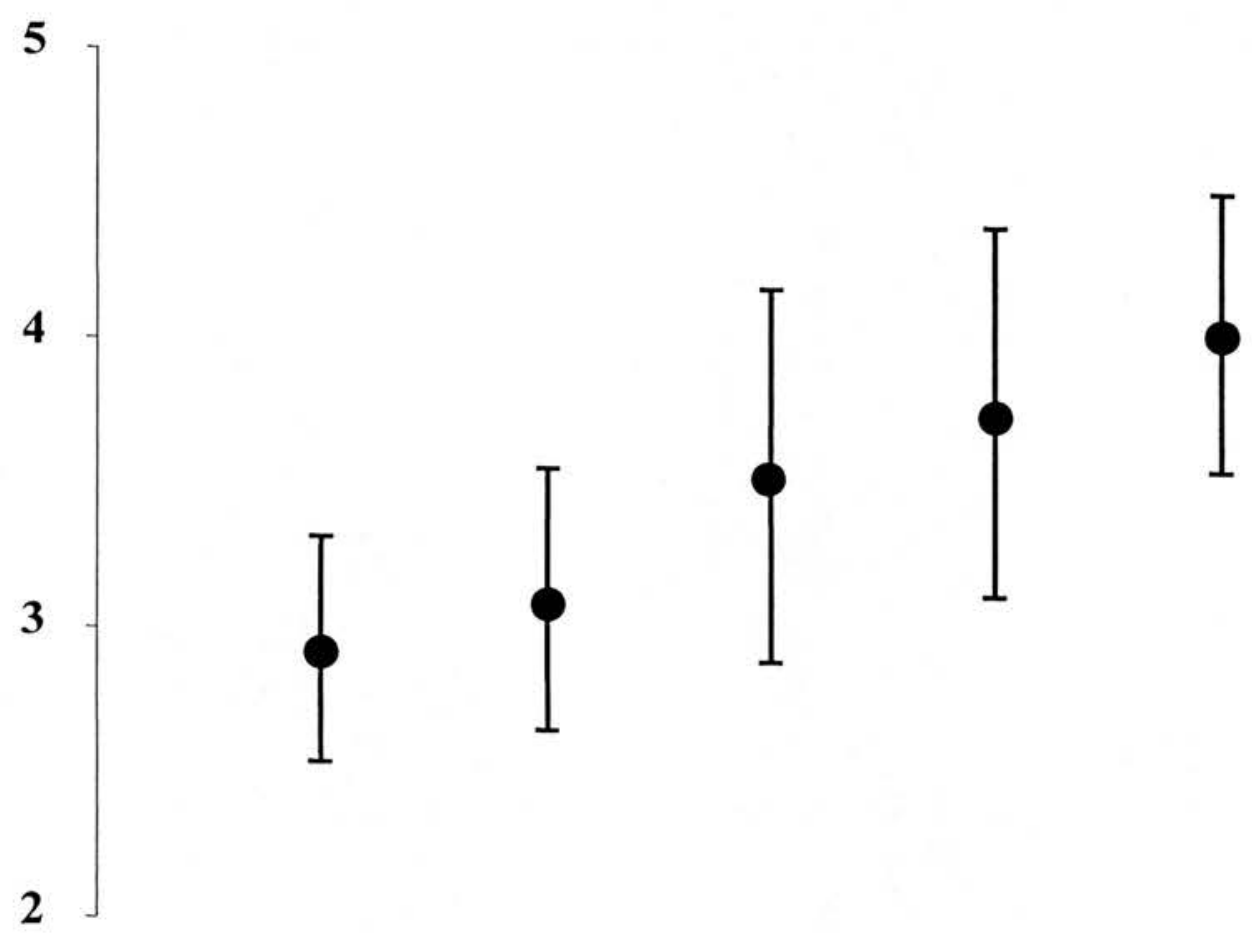

1

2

3

4

5

\section{Quintiles of Aortic Valve Calcium Score}

ANOVA, $p<0.001$. Fisher's PLSD test, $p \leq 0.03$ for all comparisons between the individual quintiles except quintile 1 versus quintile 2 , and quintile 3 versus quintile 4.

PLSD - protected least squares difference. 


\subsection{DISCUSSION}

In patients with aortic stenosis, we have demonstrated that helical CT is a reproducible means of quantifying AVC burden. We have also established a close association between the degree of aortic valve calcification and the haemodynamic severity of aortic stenosis. In particular, the presence of severe and potentially critical aortic stenosis is associated with heavy calcification. We suggest that patients found to have incidental aortic valve calcification on $\mathrm{CT}$ require further cardiological assessment for aortic stenosis, especially when there is heavy calcification.

Recent studies have evaluated electron beam CT in the quantification of AVC burden, revealing an interscan variability of less than 10\% [Pohle et al 2001; Budoff et al 2002]. Here we have confirmed that helical CT also provides accurate quantification of valvular calcification, with sufficient accuracy to evaluate progression of calcium accumulation over time. Further studies are required to determine whether different modes of CT image acquisition are comparable.

This is the first study to compare AVC scores with echocardiogram-derived measures of valvular gradients in a large number of patients with aortic stenosis. One previous small study of 19 patients also suggested that there might be an association between the severity of aortic stenosis and the valvular calcium score [Kizer et al 2001]. We have studied a much larger population with sufficient power to demonstrate a marked correlation between these parameters. However, because of the selected study 
population, these findings should only be cautiously extrapolated to aortic valve calcification identified during general population screening or as an incidental finding. A recent retrospective study suggested a $20 \%$ incidence of aortic valve calcification in over 2,000 patients attending for detection of coronary calcification [Kizer et al 2001]. One retrospective study of 109 such patients who had undergone both CT and echocardiography, reported a $30 \%$ prevalence of aortic valve calcification in which aortic stenosis was documented in 15\% [Lippert et al 1995]. In the absence of aortic valve calcification, none of the patients had significant aortic valve stenosis. In the current study, only 2 patients ( $1 \%$ ) had no detectable valvular calcification suggesting an excellent negative predictive value. Our study findings additionally suggest that the likelihood of significant valvular stenosis increases with the severity of calcification.

Echocardiography is the mainstay of clinical monitoring for aortic valve stenosis but provides only a subjective and semi-quantitative measure of aortic valve calcification. Computed tomography provides a more accurate method of quantifying calcium that more closely correlates with the aortic valve gradient than echocardiography-derived measures of calcification. It may be useful to quantify more accurately the degree of calcification given that it is the strongest independent risk factor for disease progression and an adverse clinical outcome [Rosenhek et al 2000]. Further prospective studies are now needed to assess whether the degree of calcification [Pohle et al 2001] provides useful additional clinical information that would help guide patient management. Indeed, it has been suggested that patients with severe aortic stenosis and marked calcification should undergo AVR even in the absence of symptoms [Rosenhek et al 2000]. 
Aortic valve calcification is associated with an increased cardiovascular mortality [Otto et al 1999; Rosenhek et al 2000]. The underlying pathogenetic process appears to share many of the features and risk factors for atherosclerosis [Otto et al 1994] including hypercholesterolaemia, [Wilmshurst et al 1997; Chui et al 2001] that is associated with a more rapid progression of aortic valve calcification [Pohle et al 2001]. There are several studies, including the SALTIRE trial, that are assessing the impact of lipid-lowering therapy on the rate of progression of aortic stenosis. Given that statin use is associated with halting the progression of coronary calcification [Callister et al 1998], the present study indicates that helical $\mathrm{CT}$ is a valuable method of assessing aortic valve calcification and disease progression in such intervention trials. Indeed, one preliminary observational study has suggested that statin use is associated with a lower rate of progression of aortic valve calcification [Shavelle et al 2002].

Using our methodology, we have found a close correlation between echocardiographic measures of aortic stenosis and AVC scores measured by multi-slice helical CT. The applicability of our findings to the latest CT scanners with differing specifications such as 64 slice multidetector array acquisition, or imaging parameters, such as slice thickness, pitch and ECG gating, is unknown. However, there is a high degree of agreement between different machines and coronary calcium scores [Carr et al 2000], and we believe that our findings will be applicable to other CT equipment. The reference ranges of the AVC scores are likely to be dependent on the imaging protocol and CT equipment used. However, broadly speaking, poorly defined or diffuse segments of 
calcium usually represent a minor aortic valve gradient. In contrast, coalescent calcium centred on the aortic valve is likely to represent moderate stenosis where as very heavy calcification almost invariably represents a significant degree of valvular stenosis (Figure 3.1).

In conclusion, quantification of aortic valve calcification by helical CT is a reproducible technique. Calcification of the aortic valve is closely associated with the severity of aortic stenosis and heavy calcification suggests the presence of severe aortic stenosis that requires prompt cardiological assessment. Patients with lesser degrees of aortic valve calcification should be screened for aortic stenosis and monitored for disease progression. 


\section{CHAPTER 4}

\section{INTENSIVE LIPID-LOWERING THERAPY DOES NOT HALT THE PROGRESSION OF CALCIFIC AORTIC STENOSIS}

\section{Based on}

Cowell SJ, Newby DE, Prescott RJ, Bloomfield P, Reid J, Northridge DB, Boon NA. Scottish Aortic Stenosis and Lipid-Lowering Trial, Impact on Regression (SALTIRE) Investigators. A randomised trial of intensive lipid-lowering therapy in calcific aortic stenosis. N Engl J Med 2005;352(23):2389-97. 


\subsection{SUMMARY}

Background Calcific aortic stenosis has many characteristics in common with atherosclerosis including hypercholesterolaemia. We hypothesised that intensive lipidlowering therapy would halt the progression or induce regression of calcific aortic stenosis.

Methods In this double-blind placebo-controlled trial, patients with calcific aortic stenosis were randomised to either atorvastatin $80 \mathrm{mg}$ daily or matched placebo. Aortic valve stenosis and calcification were assessed using Doppler echocardiography and helical CT respectively. The primary end-points were change in aortic jet velocity and AVC score.

Results Seventy-seven patients were assigned to atorvastatin and 78 to placebo with a median follow-up of 25 months (range 7-36). Serum low-density lipoprotein cholesterol concentration remained at $3.4 \pm 0.8 \mathrm{mmol} / \mathrm{L}$ in the placebo group, and fell to $1.7 \pm 0.6 \mathrm{mmol} / \mathrm{L}$ in the atorvastatin group $(\mathrm{p}<0.001)$. Increase in aortic jet velocity was $0.199 \pm 0.210 \mathrm{~m} / \mathrm{s}$ per year in the atorvastatin group, and $0.203 \pm 0.208 \mathrm{~m} / \mathrm{s}$ per year in the placebo group $(\mathrm{p}=0.95$ : difference $0.002 ; 95 \%$ confidence interval $(\mathrm{CI}),-0.066$ to $0.070 \mathrm{~m} / \mathrm{s} / \mathrm{yr}$ ). Progression in valvular calcification was $22.3 \pm 21.0 \%$ per year in the atorvastatin group and $21.7 \pm 19.8 \%$ per year in the placebo group $(\mathrm{p}=0.93$ : ratio of posttreatment AVC score: $0.998 ; 95 \%$ CI, 0.947 to 1.050$)$.

Conclusion This is the first double-blind randomised controlled trial of lipid-lowering therapy in patients with calcific aortic stenosis. It has clearly demonstrated that whilst 
high dose atorvastatin more than halves serum LDL cholesterol concentrations, it does not halt the progression or induce regression of the valvular disease process.

\subsection{INTRODUCTION}

In the western world, calcific aortic stenosis is the commonest form of valvular heart disease and its incidence increases with age such that $3 \%$ of adults over 75 years of age have aortic stenosis [Stewart et al 1997]. It is a gradually progressive disease, characterised by a long asymptomatic phase lasting several decades, followed by a shorter symptomatic phase associated with severe narrowing of the aortic valve orifice. Once symptoms occur, the prognosis is poor and usually mandates surgery. Calcific aortic stenosis is now the leading indication for valve replacement in North America and Europe. However, there are currently no effective disease modifying treatments and the possibility of halting, or even inducing regression of, the disease process would represent a major therapeutic advance.

Calcific aortic stenosis is mediated by a chronic active inflammatory disease process that has many similarities with atherosclerosis and includes infiltration of inflammatory cells, lipoproteins, lipids, extracellular bone matrix proteins and bone mineral [Olsson et al 1994; Otto et al 1994; O’Brien et al 1996; Olsson et al 1999]. Consistent with these observations, clinical studies have revealed a strong association with coronary artery disease [Mautner and Roberts 1992; Peltier et al 2003] and many of its risk factors including hypercholesterolaemia [Stewart et al 1997]. Disease progression in aortic 
stenosis is variable and is influenced by several factors including degree of stenosis [Otto et al 1997], valvular calcification [Davies et al 1991; Bahler et al 1999; Rosenhek et al 2000] and hypercholesterolaemia [Palta et al 2000; Nassimiha et al 2001]. Indeed, calcific aortic stenosis is a feature of severe homozygous familial hypercholesterolaemia [Rallidis et al 1998] and intensive lipid-lowering therapy with plasmapheresis has been reported to regress valvular stenosis in these patients [Keller et al 1986].

Hydroxymethylglutaryl coenzyme A reductase inhibitors, or statins, are now established treatments for the primary and secondary prevention of coronary artery disease [Shepherd et al 1995; The Heart Protection Study Collaborative Group 2002]. Several studies have shown that these drugs can halt the progression of coronary artery disease [Zhao et al 1993; Jukema et al 1995; Pitt et al 1995] as well as reduce the calcific volume of coronary plaques [Callister et al 1998; Budoff et al 2000; Achenbach et al 2002]. Given the clinical association with hypercholesterolaemia and coronary artery disease, and the striking histological similarities with atheroma, it has been suggested that statin therapy may halt the progression, or even induce regression, of calcific aortic stenosis. This hypothesis is supported by numerous retrospective observational studies [Arnow et al 2001; Novaro et al 2001; Pohle et al 2001; Bellamy et al 2002; Shavelle et al 2002; Rosenhek et al 2004] showing that concomitant statin therapy was associated with a delay in the progression of the aortic jet velocity of $0.30 \mathrm{~m} / \mathrm{s}$ per year and calcification by $30 \%$ per year. 
The aim of the SALTIRE trial was to establish whether intensive lipid-lowering therapy with atorvastatin $80 \mathrm{mg}$ daily would halt the progression, or induce regression, of the aortic jet velocity on Doppler echocardiography, and the AVC score on CT, in patients with calcific aortic stenosis.

\subsection{METHODS}

The SALTIRE trial was a randomised double-blind placebo-controlled trial. The investigation conformed to the Declaration of Helsinki, and all participating regional ethics committees approved the study protocol. All patients gave written informed consent.

\subsubsection{Patient POPUlation}

Patients from the south-east of Scotland attending seven district and two regional centres were approached for enrolment by the study co-ordinator between December 2001 and April 2002. Eligible individuals were identified from outpatient clinics and echocardiography databases. Those aged over 18, with calcific aortic stenosis, an aortic jet velocity of $\geq 2.5 \mathrm{~m} / \mathrm{s}$, and grade 1-3 calcification of the aortic valve on echocardiography [Rosenhek et al 2000] were included. Exclusion criteria are detailed in Chapter 2. Of the patients screened, 455 were eligible for inclusion, 173 agreed to participate and 155 were ultimately randomised to treatment. 


\subsubsection{STUDY PROTOCOL}

Between March 2001 and the end of April 2002, the blinded study co-ordinator randomised 155 eligible patients by the minimisation technique [Treasure and MacRae 1998] using a dedicated locked computer programme (Edinburgh University) which incorporated eight baseline variables: age, sex, smoking habit, hypertension, diabetes mellitus, serum cholesterol concentration, aortic jet velocity, and AVC score. Patients were assigned to either atorvastatin $80 \mathrm{mg}$ daily or matched placebo (Pfizer Ltd, UK) as a single daily dose using numbered containers.

Patients were assessed at baseline, 2 months, 6 months and every 6 months thereafter for between 2 and 3 years. Clinical evaluation included assessment of functional status and adverse events as well as blood analysis of renal function, liver function, creatine kinase and lipid profile. Echocardiography and CT were performed at baseline, at each annual visit and prior to withdrawal from the study. Randomised patients who were subsequently commenced on open label statin therapy by their attending physician were immediately scanned and then withdrawn from the study.

\subsubsection{Echocardiography}

Assessment of valvular stenosis was determined by a single dedicated research ultrasonographer. All scans were performed on one of two dedicated ultrasound machines Vingmed System 5 Performance (BMS (Scotland) Ltd, Belshill, UK), or ATL3000 cardiac ultrasound machine (Philips Medical Systems (UK) Ltd, Stevenage, UK) 
that were maintained constant for each patient throughout the study. Patients were studied using a 3-MHz transducer for $\mathrm{M}$-mode, and two-dimensional imaging with integral pulsed and continuous wave Doppler. All measurements were determined online, averaged from three cardiac cycles (five cycles if in atrial fibrillation), and recorded onto Super-VHS video and optical disk using a standardised proforma. Aortic jet velocity was recorded from the window generating the highest signal (apical, right parasternal or suprasternal). Left ventricular outflow tract velocity was measured from an apical approach just proximal to the aortic valve leaflets. Peak and mean aortic valve gradients were calculated using the Bernoulli equation, and AVA using the continuity equation. The left ventricular outflow tract diameter was measured at baseline and maintained constant throughout the study.

\subsubsection{Computed tomography}

Computed tomography was performed by a single operator using a double-helix scanner (Twin II Flash; Philips Medical Systems (UK) Ltd, Stevenage, UK) and calibrated against a standard phantom. The region of the aortic valve was imaged with a spiral acquisition using $2.7 \mathrm{~mm}$ slices, with a pitch of 0.7 and an increment of $1.4 \mathrm{~mm}$ during held inspiration. All images were analysed by a single operator using an automated computerised software program (Picker Cardiac Scoring). This employs a modified Agatston scoring method [Shemesh et al 1995] that uses a threshold of $90 \mathrm{HU}$ to compensate for non-gated imaging. 
Reproducibility of echocardiographic and CT assessments was determined in two subsets of 20 patients as described in Chapter 3 [Cowell et al 2003]. Coefficients of reproducibility for aortic jet velocity and AVC score were $0.32 \mathrm{~m} / \mathrm{s}$ and $0.07 \mathrm{pAU}$ respectively [Cowell et al 2003].

\subsubsection{DATA AND STATISTICAL ANALYSIS}

The study was designed to assess the two primary end-points: progression of stenosis determined by change in aortic jet velocity on Doppler echocardiography, and progression of valvular calcification measured by CT. Secondary end-points were a composite clinical end-point (cardiovascular mortality, AVR) or hospitalisation attributable to severe aortic stenosis), AVR, all cause hospitalisation and cardiovascular hospitalisation. The planned sample size of 75 patients per group gave $80 \%$ power at a $5 \%$ significance level to detect a difference in the primary end-points of $0.15 \mathrm{~m} / \mathrm{s}$ per year in aortic jet velocity [Faggiano et al 1992; Otto et al 1997] and 500 AU in the AVC score [Shemesh et al 1995].

The Data Monitoring Committee conducted two interim assessments of safety, as well as an interim assessment of efficacy one year after randomisation. The trial was to be terminated early in the event of a negative treatment effect $(\mathrm{p}<0.05)$, or strong treatment benefit $(\mathrm{p}<0.001)$. On the recommendation of the Data Monitoring Committee, the trial continued until study completion. 
Analyses were performed by RP using SPSS Version 12.0. All outcome variables were analysed by intention-to-treat. Disease progression was determined by calculating the rate of change between the baseline and final scans. Treatment comparisons for the continuous outcome variables were based on an analysis of covariance, with the prerandomisation level of that variable used as a covariate. In the subgroup analyses, interaction terms between treatment and subgroup have been added to a model incorporating pre-randomisation level, treatment and subgroup to assess whether there is any evidence of a differential treatment effect in subgroups. Categorical variables have been analysed using Fisher's exact test. Two-tailed tests are employed throughout. Statistical significance was taken as $\mathrm{p}<0.05$.

\subsection{RESULTS}

Seventy-seven patients were assigned to atorvastatin and 78 to placebo with a median follow-up of 25 months (range 7-36) (Figure 4.1). As a consequence of minimisation, the baseline characteristics were well matched (Table 4.1). All patients had good left ventricular function except one patient who had mild impairment of systolic function. Mean aortic jet velocity was $3.43 \pm 0.64$ (range $2.5-5.0$ ) $\mathrm{m} / \mathrm{s}$, and median AVC score was 5920 (interquartile range 2485-14231) AU. There were 119 patients with mild to moderate aortic stenosis (aortic jet velocity, 2.5 to $3.9 \mathrm{~m} / \mathrm{s}$ ), and 36 with severe stenosis (aortic jet velocity, $\geq 4.0 \mathrm{~m} / \mathrm{s}$ ). 


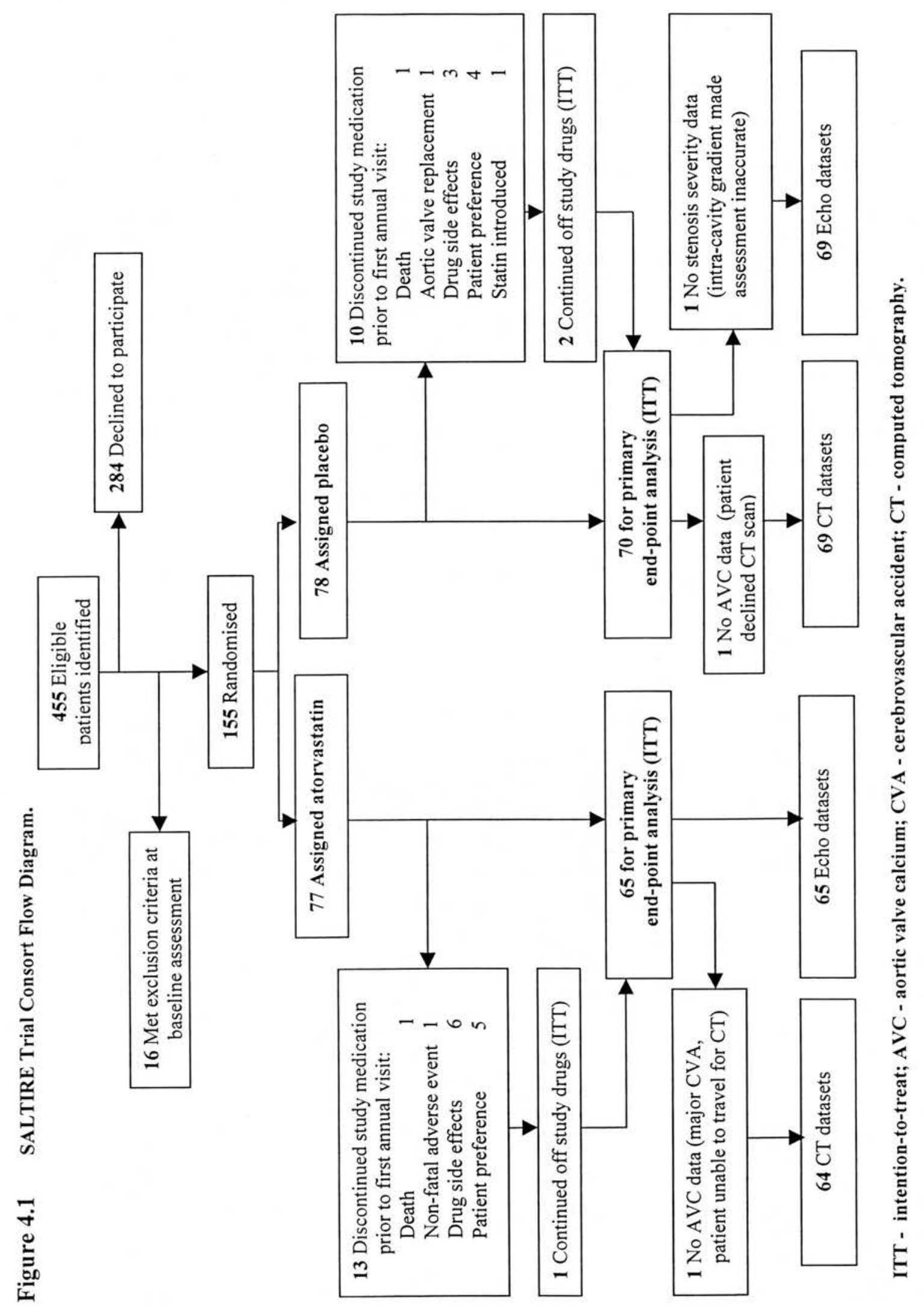




\section{TABLE 4.1 Baseline characteristics}

\begin{tabular}{|c|c|c|}
\hline & $\begin{array}{c}\text { Atorvastatin } \\
\mathrm{n}=77\end{array}$ & $\begin{array}{c}\text { Placebo } \\
\mathrm{n}=78\end{array}$ \\
\hline \multicolumn{3}{|l|}{ Demographics } \\
\hline Age (years) & $68(11)$ & $68(10)$ \\
\hline Sex (\% male) & 68 & 72 \\
\hline \multicolumn{3}{|l|}{ Cardiovascular Risk Factors } \\
\hline Hypertension & 48 & 54 \\
\hline Hyperlipidaemia & 8 & 5 \\
\hline Diabetes mellitus & 3 & 4 \\
\hline Current smoker & 21 & 22 \\
\hline \multicolumn{3}{|l|}{ Cardiovascular Disease } \\
\hline Coronary heart disease & 18 & 21 \\
\hline Cerebrovascular disease & 9 & 11 \\
\hline Peripheral vascular disease & 5 & 13 \\
\hline \multicolumn{3}{|l|}{ Drug History } \\
\hline Aspirin & 43 & 40 \\
\hline ACE inhibitor & 12 & 14 \\
\hline Beta-blocker & 21 & 27 \\
\hline Warfarin & 8 & 12 \\
\hline \multicolumn{3}{|l|}{ Physical Examination } \\
\hline Height $(\mathrm{cm})$ & $168(9)$ & $169(8)$ \\
\hline Weight $(\mathrm{Kg})$ & $79(15)$ & $80(15)$ \\
\hline Heart rate $(\mathrm{bpm})$ & $68(11)$ & $66(12)$ \\
\hline Systolic blood pressure (mmHg) & $144(18)$ & $144(21)$ \\
\hline Diastolic blood pressure (mmHg) & $82(10)$ & $81(12)$ \\
\hline \multicolumn{3}{|l|}{ Biochemistry } \\
\hline Total cholesterol $(\mathrm{mmol} / \mathrm{L})$ & $5.8(1.0)$ & $5.7(0.9)$ \\
\hline LDL cholesterol $(\mathrm{mmol} / \mathrm{L})$ & $3.6(0.9)$ & $3.5(0.8)$ \\
\hline Cholesterol:HDL ratio & $4.1(1.1)$ & $4.1(1.4)$ \\
\hline Urea $(\mathrm{mmol} / \mathrm{L})$ & $6.1(2.1)$ & $6.9(6.8)$ \\
\hline Creatinine $(\mu \mathrm{mol} / \mathrm{L})$ & $91(21)$ & $92(22)$ \\
\hline Glucose $(\mathrm{mmol} / \mathrm{L})$ & $5.2(1.1)$ & $5.4(1.2)$ \\
\hline \multicolumn{3}{|l|}{ Electrocardiogram } \\
\hline Sinus rhythm & 94 & 92 \\
\hline Atrial fibrillation & 6 & 8 \\
\hline Romhilt-Estes score $\dagger$ & $1(0-3)$ & $2(1-4)$ \\
\hline \multicolumn{3}{|l|}{ Echocardiography } \\
\hline Tricuspid aortic valve & 96 & 97 \\
\hline Bicuspid aortic valve & 4 & 3 \\
\hline Aortic jet velocity $(\mathrm{m} / \mathrm{s})$ & $3.39(0.62)$ & $3.45(0.67)$ \\
\hline Peak gradient $(\mathrm{mmHg})$ & $47.8(17.4)$ & $49.5(19.5)$ \\
\hline Aortic valve area $\left(\mathrm{cm}^{2}\right)$ & $1.03(0.4)$ & $1.02(0.41)$ \\
\hline \multicolumn{3}{|l|}{ Computed Tomography } \\
\hline Aortic valve calcium score $\dagger$ & $5424(2750-9689)$ & $6221(3037-9575)$ \\
\hline Log aortic valve calcium score ( $\log A U)$ & $3.7(0.5)$ & $3.7(0.6)$ \\
\hline
\end{tabular}




\subsubsection{SERUM CHOLESTEROL CONCENTRATIONS}

The mean serum low-density lipoprotein cholesterol concentration remained at $3.4 \pm 0.8 \mathrm{mmol} / \mathrm{L}$ in the placebo group, and fell to a mean on treatment of $1.7 \pm 0.6 \mathrm{mmol} / \mathrm{L}$ in the atorvastatin group $(\mathrm{p}<0.001)$. This equates to a $53 \%$ reduction in LDL cholesterol in the atorvastatin group (Figure 4.2c). Serum total cholesterol was $5.5 \pm 0.9 \mathrm{mmol} / \mathrm{L}$ and $3.5 \pm 0.7 \mathrm{mmol} / \mathrm{L}$ in the placebo and atorvastatin groups respectively $(\mathrm{p}<0.001)$ and is in keeping with pill count compliance that averaged $97 \%$ in both treatment groups.

\subsubsection{IMPACT OF ATORVASTATIN ON DISEASE PROGRESSION}

Intensive lipid-lowering therapy with atorvastatin $80 \mathrm{mg}$ daily had no effect on the rate of change in aortic jet velocity or valvular calcification (Table 4.2; Figure 4.2). Serum LDL cholesterol concentrations did not correlate with disease progression on echocardiography $(\mathrm{r}=0.021, \mathrm{p}=0.81)$ or $\mathrm{CT}(\mathrm{r}=-0.109, \mathrm{p}=0.21)$ (Figure 4.3). The proportion of patients reaching secondary clinical end-points appeared to be fewer in the atorvastatin group but none of the comparisons achieved statistical significance (Table 4.3).

\subsubsection{SUBGROUP ANALYSES}

Pre-specified subgroup analysis of the primary end-point data was conducted in patients with mild to moderate (aortic jet velocity of $<4.0 \mathrm{~m} / \mathrm{s}$ ) and severe (aortic jet velocity $\geq 4.0 \mathrm{~m} / \mathrm{s}$ ) aortic stenosis at baseline. As anticipated from earlier studies, patients with 
Figure 4.2 Progression in aortic valve stenosis and serum LDL cholesterol concentrations in patients treated with atorvastatin $80 \mathrm{mg}$ daily (solid circles) and matched placebo (open squares).
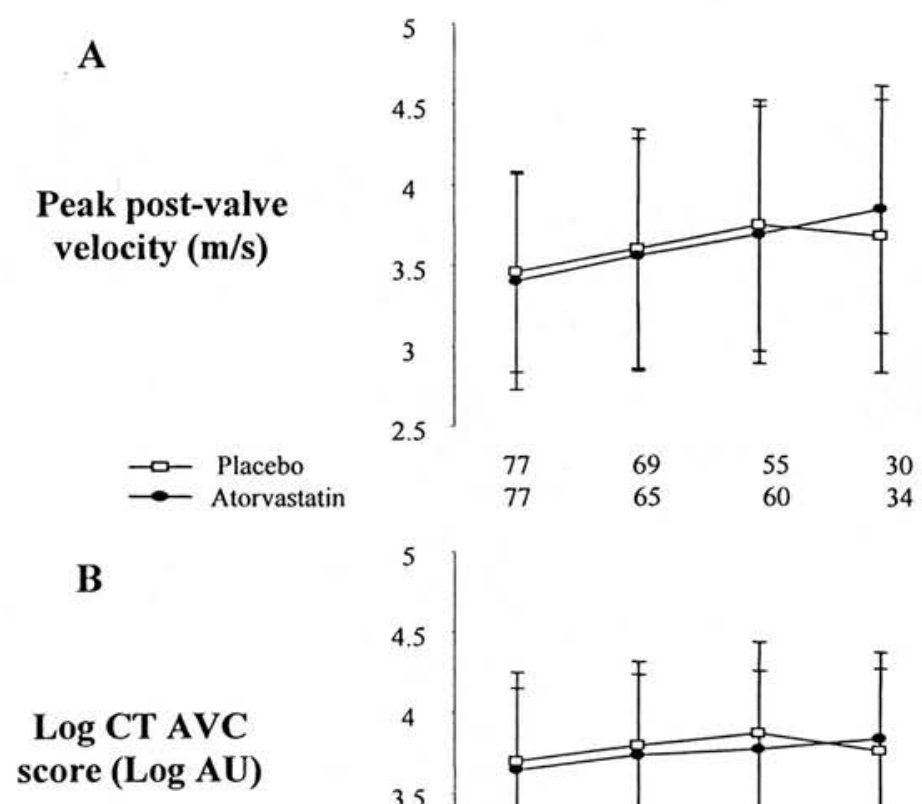

B

$$
\begin{gathered}
\log \text { CT AVC } \\
\text { score }(\log A U)
\end{gathered}
$$

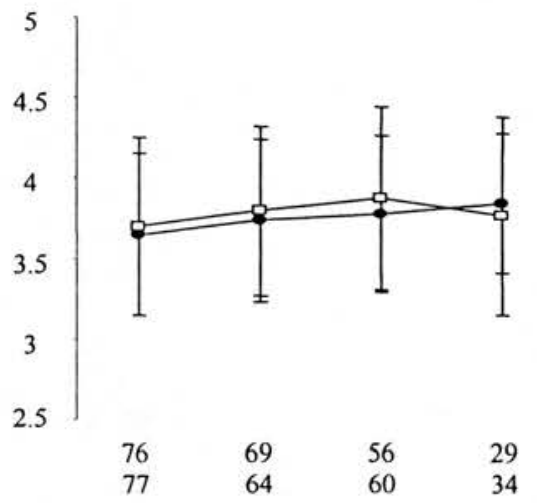

C

LDL cholesterol $(\mathrm{mmol} / \mathrm{L})$
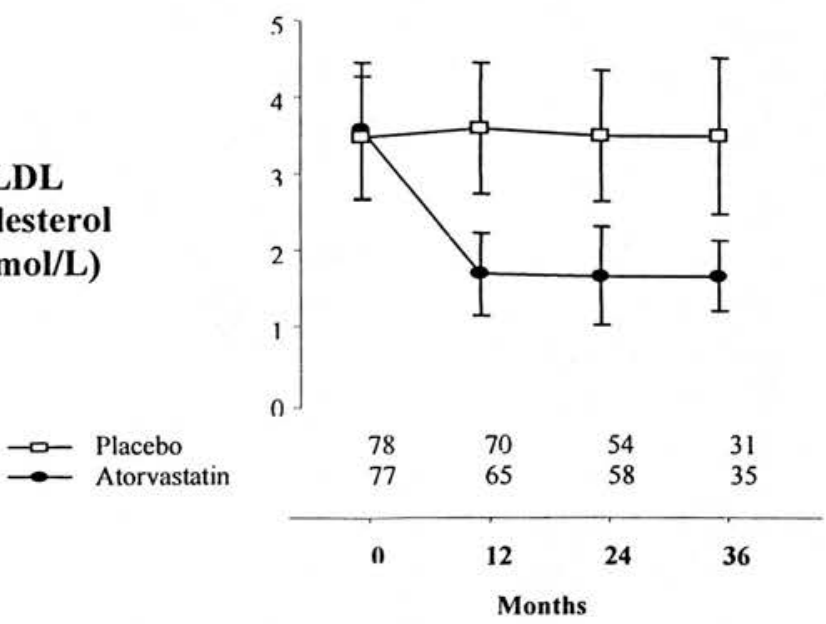

LDL - low-density lipoprotein; CT - computed tomography; AVC - aortic valve calcium; Log - logarithm; AU - arbitrary units. 


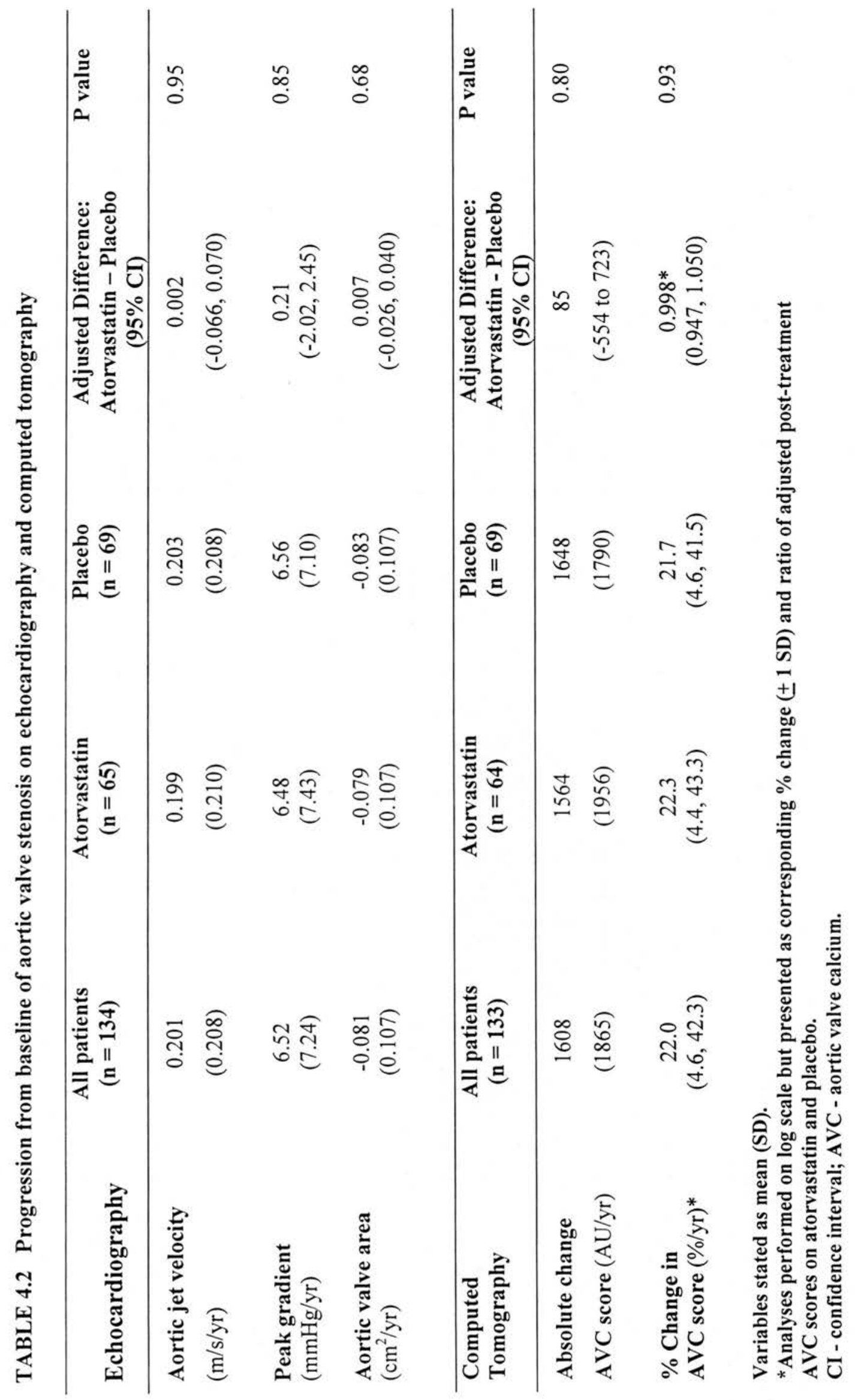


Figure 4.3 Relationship between LDL cholesterol concentration and disease progression.
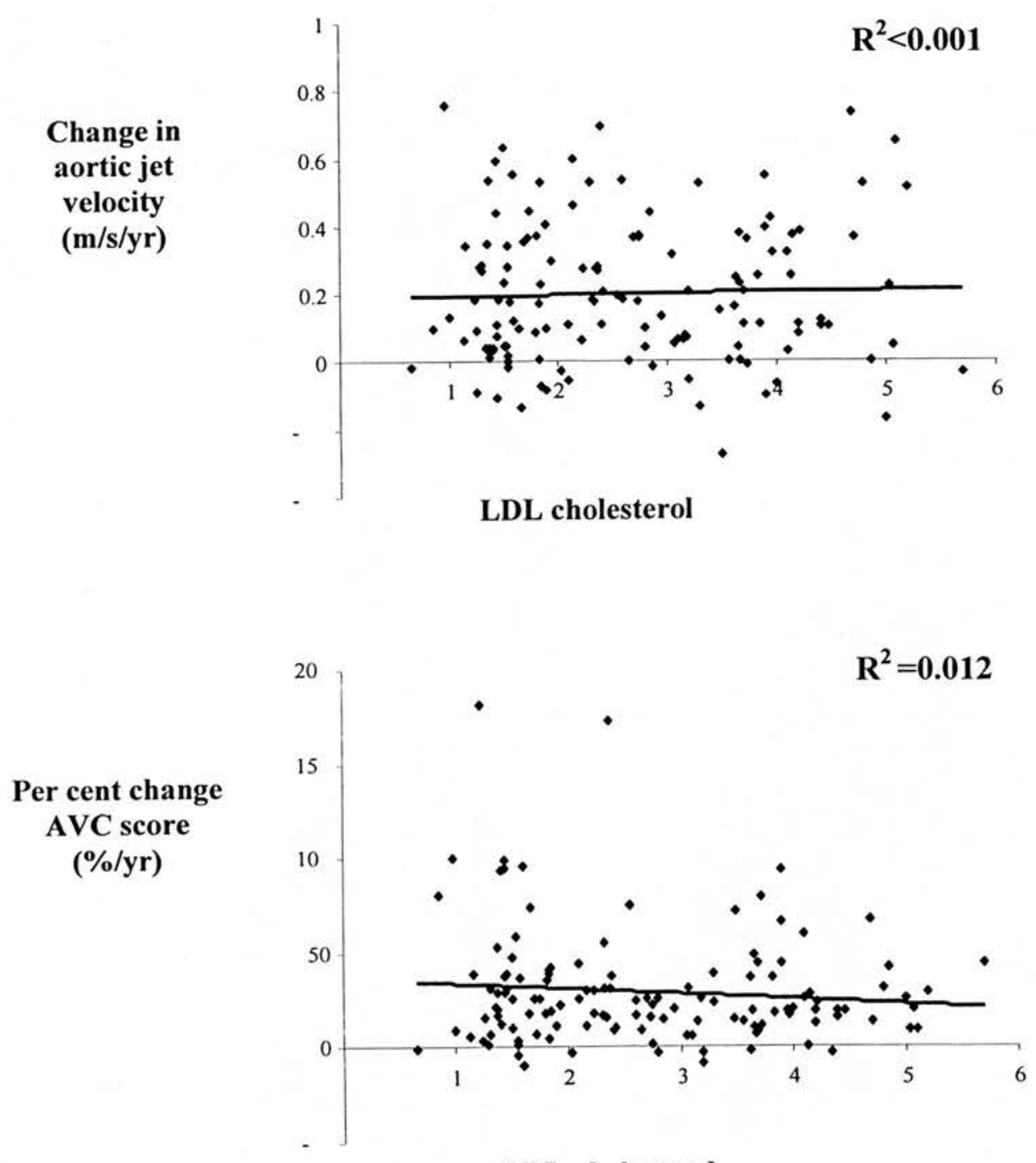

LDL cholesterol

LDL - low-density lipoprotein; AVC - aortic valve calcium. 
TABLE 4.3 Secondary end-points

\section{Atorvastatin Placebo P value \\ $(n=77) \quad(n=78) \quad$ (Fisher's Exact Test)}

Composite secondary end-point

13

21

0.19

Hospitalisation for severe aortic stenosis

3

5

0.73

Aortic valve replacement

11

19

0.17

Cardiovascular mortality

3

3

1.00

All cause mortality

3

5

0.73

All cardiac admissions

10

12

0.84 
severe stenosis at baseline progressed more rapidly $(\mathrm{p}=0.04)$, but the study findings were consistent irrespective of baseline stenosis severity (Table 4.4). Likewise, length of follow-up did not influence outcome. In those followed up for $>24$ months, increase in aortic jet velocity was $0.21 \pm 0.20 \mathrm{~m} / \mathrm{s}$ per year in the atorvastatin group and $0.17 \pm 0.14 \mathrm{~m} / \mathrm{s}$ per year in the placebo group (Table 4.4 ).

\subsubsection{ADVERSE EVENTS}

High dose atorvastatin was well tolerated: the frequency of adverse events was similar in the two treatment groups. Discontinuation of the study drug occurred in 4 patients $(5 \%)$ of the placebo group and 7 patients $(9 \%)$ of the atorvastatin group (Fisher's exact test, $\mathrm{p}=0.52$ ), predominantly as a result of gastrointestinal symptoms. Three patients in the atorvastatin group experienced an increase in creatine kinase to greater than five times the upper limit of normal without symptoms of myositis; one of whom was withdrawn at the request of the Data Monitoring Committee. There were no cases of rhabdomyolysis and no serious adverse events. 
TABLE 4.4 Subgroup analyses of disease progression

\begin{tabular}{|c|c|c|c|c|}
\hline \multicolumn{2}{|c|}{ Baseline severity of stenosis: } & Atorvastatin & Placebo & \\
\hline Mild to moderate & $\begin{array}{c}\text { Baseline } \\
(\mathrm{m} / \mathrm{s})\end{array}$ & $\begin{array}{c}3.12(0.43) \\
\mathrm{n}=58\end{array}$ & $\begin{array}{c}3.18(0.44) \\
n=61\end{array}$ & \multirow{4}{*}{$\begin{array}{c}\text { Interaction } \\
\quad \text { test: } \\
\mathbf{p}=\mathbf{0 . 5 7}\end{array}$} \\
\hline $\begin{array}{l}\text { (Baseline aortıc jet } \\
\text { velocity }<4 \mathrm{~m} / \mathrm{s} \text { ) }\end{array}$ & $\begin{array}{c}\text { Rate of change } \\
(\mathrm{m} / \mathrm{s} / \mathrm{yr})\end{array}$ & $\begin{array}{c}0.17(0.21) \\
n=49\end{array}$ & $\begin{array}{c}\mathbf{0 . 1 9}(\mathbf{0 . 2 0}) \\
\mathrm{n}=55\end{array}$ & \\
\hline \multirow{2}{*}{$\begin{array}{c}\text { Severe } \\
\text { (Baseline aortic jet } \\
\text { velocity } \geq 4 \mathrm{~m} / \mathrm{s} \text { ) }\end{array}$} & $\begin{array}{c}\text { Baseline } \\
(\mathrm{m} / \mathrm{s})\end{array}$ & $\begin{array}{c}4.24(0.21) \\
\mathrm{n}=19\end{array}$ & $\begin{array}{c}4.45(0.26) \\
\mathrm{n}=17\end{array}$ & \\
\hline & $\begin{array}{c}\text { Rate of change } \\
(\mathrm{m} / \mathrm{s} / \mathrm{yr})\end{array}$ & $\begin{array}{c}0.27(0.21) \\
\mathrm{n}=16\end{array}$ & $\begin{array}{c}0.27(0.23) \\
\mathrm{n}=14\end{array}$ & \\
\hline \multicolumn{2}{|c|}{ Follow-up duration: } & Atorvastatin & Placebo & \\
\hline \multirow{2}{*}{$\begin{array}{c}\text { Follow-up } \\
\leq \mathbf{2 4} \text { months } \\
\text { (Median } 23 \text { months) }\end{array}$} & $\begin{array}{c}\text { Baseline } \\
(\mathrm{m} / \mathrm{s})\end{array}$ & $\begin{array}{c}3.49(0.69) \\
\mathrm{n}=30\end{array}$ & $\begin{array}{c}3.64(0.67) \\
\mathrm{n}=37\end{array}$ & \multirow{4}{*}{$\begin{array}{c}\text { Interaction } \\
\quad \text { test: } \\
p=0.41\end{array}$} \\
\hline & $\begin{array}{c}\text { Rate of change } \\
(\mathrm{m} / \mathrm{s} / \mathrm{yr})\end{array}$ & $\begin{array}{c}0.19(0.22) \\
n=30\end{array}$ & $\begin{array}{c}0.23(0.25) \\
n=37\end{array}$ & \\
\hline \multirow{2}{*}{$\begin{array}{c}\text { Follow-up } \\
>\mathbf{2 4} \text { months } \\
\text { (Median } 33 \text { months) }\end{array}$} & $\begin{array}{c}\text { Baseline } \\
(\mathrm{m} / \mathrm{s})\end{array}$ & $\begin{array}{c}3.31(0.55) \\
\mathrm{n}=35\end{array}$ & $\begin{array}{c}3.28(0.61) \\
n=32\end{array}$ & \\
\hline & $\begin{array}{l}\text { Rate of change } \\
(\mathrm{m} / \mathrm{s} / \mathrm{yr})\end{array}$ & $\begin{array}{c}0.21(0.20) \\
n=35\end{array}$ & $\begin{array}{c}0.17(0.14) \\
n=32\end{array}$ & \\
\hline
\end{tabular}

Mean (SD) 


\subsection{DISCUSSION}

This is the first double-blind randomised controlled trial of lipid-lowering therapy in patients with calcific aortic stenosis. It has clearly demonstrated that whilst high dose atorvastatin more than halves serum LDL cholesterol concentrations, it does not halt the progression or induce regression of the valvular disease process. This has been demonstrated using two distinct measures of disease severity: aortic jet velocity determined by Doppler echocardiography and valvular calcification by helical CT. Moreover, there was no relationship between serum LDL cholesterol concentrations and the progression of aortic stenosis, nor was there a demonstrable effect of high dose atorvastatin on clinical end-points. Thus, irrespective of the method of assessing disease progression, we have consistently demonstrated the continued deterioration of aortic stenosis despite intensive reductions in serum cholesterol concentrations.

In this trial, there was a single co-ordinating centre with a consistent and reproducible approach to assessing the severity of aortic stenosis [Cowell et al 2003]. High dose atorvastatin therapy achieved the anticipated dramatic reduction in serum LDL cholesterol concentrations [Jones et al 1998], and disease progression was determined using two independent but complementary techniques. The trial employed a doubleblind randomisation incorporating the minimisation technique to ensure no baseline inequalities between the treatment groups but, despite this rigorous methodology, we were unable to demonstrate a major impact on disease progression in these patients. 
Several factors may have influenced our ability to detect an effect of statin therapy on the progression of aortic stenosis in this trial.

First, as a consequence of our inclusion criteria, we recruited some patients with advanced disease and an aortic jet velocity of $\geq 4 \mathrm{~m} / \mathrm{s}$ and it could be argued that lipidlowering therapy is unlikely to influence such an advanced stage of the disease. We therefore conducted a pre-specified subgroup analysis excluding patients with a baseline aortic jet velocity $\geq 4 \mathrm{~m} / \mathrm{s}$. Our findings were consistent irrespective of baseline stenosis severity and atorvastatin had no effect on disease progression even in the majority of individuals with mild to moderate stenosis. We excluded patients with aortic jet velocities below $2.5 \mathrm{~m} / \mathrm{s}$ and we acknowledge that intervening at this earlier stage of the disease process may have been more beneficial. However, such patients do not commonly present to routine clinical practice.

Second, the length of treatment may have been inadequate and 2 years may not have been sufficient to influence the natural history of the disease. We assessed this possibility by determining if patients with longer-term follow-up demonstrated a treatment benefit. In patients maintained on nearly 3 years of treatment with intensive statin therapy, no trend towards a beneficial effect of atorvastatin was apparent. We therefore do not believe that the absence of an effect was due to an inadequate treatment period. 
Finally, although this is the largest randomised controlled trial to date, our study was only designed to detect a substantial delay in disease progression and was not powered to assess meaningful effects on clinical end-points, such as valve replacement and cardiovascular death. Whilst we can exclude a treatment benefit of the magnitude previously reported in retrospective observational studies (aortic jet velocity, $0.30 \mathrm{~m} / \mathrm{s} / \mathrm{yr}$ [Rosenhek et al 2004] and valvular calcification, 30\% per year [Pohle et al 2001; Shavelle et al 2002]), the $95 \%$ confidence intervals indicate that we may have missed a modest treatment benefit (a delay in disease progression of $<0.07 \mathrm{~m} / \mathrm{s} / \mathrm{yr}$, and $<5 \% / \mathrm{yr}$ respectively). Although such modest reductions are unlikely to be meaningful in the majority of older patients, a small decrease in disease progression may be clinically important in younger patients with mild disease who may progress over many years.

Given the strength of the data linking aortic stenosis with atherosclerosis and hypercholesterolemia, why have we have failed to halt the progression of calcific aortic stenosis? One potential explanation is that, whilst these features may drive the initiation of aortic stenosis, disease progression may be dependent upon other factors. The aortic valve is subject to continuous dynamic mechanical stress, and leaflet plasticity and structure can have an overriding influence, such as with a bicuspid valve. Moreover, in contrast to atherosclerosis, aortic stenosis is associated with a virtual absence of smooth muscle cell proliferation and lipid-laden macrophages [Otto et al 1994], and dominated by earlier and more extensive mineralisation. Decreasing the lipid pool and increasing the fibrous cap may be less relevant to the progression of aortic stenosis than it is for the 
reduction in atherosclerotic plaque rupture with statin therapy in patients with coronary heart disease.

Statin therapy in patients with aortic stenosis may confer secondary preventative benefits that are independent of its effects on the valvular disease process because of the association between aortic stenosis and coronary artery disease. The current study was not powered to assess the benefits of lipid-lowering therapy on cardiovascular endpoints such as non-fatal and fatal myocardial infarction. It remains a possibility that aortic stenosis and sclerosis [Otto et al 1999] may be important markers of occult vascular disease and thereby identify patients who would gain from the preventative benefits of statin therapy.

We conclude that intensive lipid-lowering therapy with atorvastatin $80 \mathrm{mg}$ daily does not halt the progression, or induce the regression, of calcific aortic stenosis. Nevertheless, this trial does not exclude a modest reduction in the rate of disease progression or a significant reduction in major clinical end-points. Our study reinforces the need for a long-term large scale randomised controlled trial of intensive lipidlowering therapy in patients with calcific aortic stenosis. In the meantime we do not recommend statin therapy for patients with calcific aortic stenosis in the absence of coexisting vascular disease. 
CHAPTER 5

\title{
PROGRESSIVE CORONARY CALCIFICATION DESPITE INTENSIVE LIPID-LOWERING THERAPY: \\ A RANDOMISED CONTROLLED TRIAL
}

\begin{abstract}
Based on
Houslay ES, Cowell SJ, Prescott RJ, Reid J, Burton J, Northridge DB, Boon NA, Newby DE, Scottish Aortic Stenosis and Lipid-Lowering Therapy, Impact on Regression Trial Investigators. Progressive coronary calcification despite intensive lipid-lowering treatment: a randomised controlled trial. Heart 2006;92(9):1207-12.
\end{abstract}




\subsection{SUMMARY}

Background Observational studies have suggested that statin therapy may induce regression of coronary artery calcification. In a substudy of a trial recruiting patients with calcific aortic stenosis, we evaluated the effect of intensive lipid-lowering therapy on coronary artery calcification.

Methods In a double-blind randomised controlled trial, 102 patients with calcific aortic stenosis and coronary artery calcification were randomised using the minimisation technique to atorvastatin $80 \mathrm{mg}$ daily or matched placebo. Coronary artery calcification was assessed annually by helical CT.

Results Forty-eight patients were randomised to atorvastatin and 54 to placebo with a median follow-up of 24 months (interquartile range 24-30). Baseline characteristics and coronary artery calcium scores were similar in both groups. Atorvastatin therapy reduced serum low-density lipoprotein cholesterol $(-53 \% ; \mathrm{p}<0.001)$ and CRP $(-49 \%$; $\mathrm{p}<0.001)$ concentrations whilst there was no change with placebo $(-7 \%$ and $+17 \%$; $p>0.95$ for both). The rate of change in coronary artery calcification was $26 \%$ per year (0.234 (standard error (SE) 0.037) $\log \mathrm{AU} / \mathrm{yr} ; \mathrm{n}=39)$ in the atorvastatin group and $18 \%$ per year (0.167 (SE 0.034) log AU/yr; $n=49)$ in the placebo group: geometric mean difference of $+7 \%$ per year $(95 \%$ CI $-3 \%$ to $+18 \%$; $p=0.18)$. There was no correlation between serum low-density lipoprotein concentrations and the rate of progression of coronary calcification $(\mathrm{r}=0.05, \mathrm{p}=0.62)$.

Conclusion In contrast to previous observational studies, this randomised controlled trial has shown that, despite reducing systemic inflammation and halving serum 
low-density lipoprotein cholesterol concentrations, statin therapy does not have a major effect on the rate of progression of coronary artery calcification.

\subsection{INTRODUCTION}

Coronary artery calcification is an independent risk factor for coronary heart disease with even low coronary calcium scores doubling the risk of coronary events [Pletcher et al 2004]. The relative risk associated with coronary calcification is greater than that associated with established factors such as smoking, hypertension and diabetes mellitus. Progression of coronary artery calcification is associated with a higher incidence of coronary events even in those people who are asymptomatic at the time of initial scanning [Raggi et al 2003] Thus, the presence of coronary artery calcification is not only indicative of atheromatous plaque disease, but its progression may correspond with cardiovascular event rates.

Statin therapy has a proven role in the primary [Shepherd et al 1995; Downs et al 1998] and secondary prevention [The Scandinavian Simvastatin Survival Study (4S) 1994; Lewis et al 1998; The LIPID Study Group 1998; The Heart Protection Study Collaborative Group 2002] of cardiovascular disease with incremental benefits seen with more intensive reductions in serum cholesterol concentrations [The Heart Protection Study Collaborative Group 2002]. Previous studies [Callister et al 1998; Achenbach et al 2002] have reported that statins can halt the progression and may even induce regression of coronary artery calcification. Indeed, the rate of progression of coronary 
artery calcification correlates with the average serum LDL cholesterol concentration [Callister et al 1998]. This has led to the use of CT to monitor disease progression and response to treatment, particularly statin therapy. However, two recent trials have failed to demonstrate a benefit of statin therapy on the progression of coronary artery calcification in asymptomatic individuals [Arad et al 2005; Raggi et al 2005].

The Scottish Aortic Stenosis Lipid lowering Therapy, Impact on REgression trial was a prospective double-blind randomised controlled study of intensive lipid-lowering therapy in patients with calcific aortic stenosis [Cowell et al 2005]. As part of this trial, aortic valve and coronary artery calcium scores are measured using helical CT. The aim of this substudy was to assess the effect of atorvastatin $80 \mathrm{mg}$ daily on the rate of progression of coronary artery calcification in patients with calcific aortic stenosis.

\subsection{METHODS}

\subsubsection{Patient population}

Patients aged $>18$ years, with calcific aortic stenosis (grade 1-3 calcification on echocardiography [Rosenhek et al 2000]) and a peak post-valve velocity of $\geq 2.5 \mathrm{~m} / \mathrm{s}$ were recruited from eight hospital centres across the South East of Scotland. Exclusion criteria are outlined in Chapter 2. For the purposes of the substudy, we also excluded patients who had no coronary artery calcification on CT. The study was conducted with the approval of all regional research ethics committees and in accordance with the Declaration of Helsinki. Written informed consent was obtained from each subject. 


\subsubsection{STUDY PROTOCOL}

Between March 2001 and April 2002, the blinded study co-ordinator randomised eligible patients by the minimisation technique [Treasure and MacRae 1998] using a dedicated locked computer program (Edinburgh University) which incorporated eight baseline variables: age, sex, smoking habit, hypertension, diabetes mellitus, serum cholesterol concentration, peak aortic jet velocity and aortic calcium score. Patients were assigned either to atorvastatin $80 \mathrm{mg}$ daily or matched placebo (Pfizer Ltd., Tadworth, UK) as a single daily dose using numbered containers.

Patients were assessed at baseline, 2 months, 6 months and every 6 months thereafter for a minimum of 2 years. Clinical evaluation included assessment of functional status, adverse events and biochemical blood analysis. Serum highly sensitive C-reactive protein (hsCRP) concentrations were determined using a highly sensitive immunonephelometric method (Dade Behring Ltd, Milton Keynes, UK) as previously described [Carr et al 2000]. All patients underwent CT within the month before randomisation to study therapy and at each annual visit. Randomised patients who were subsequently commenced on open label statin therapy by their attending physician were immediately scanned and withdrawn from further observation.

\subsubsection{COMPUTED TOMOGRAPHY}

Computed tomography was performed by a single-blinded operator using a double-helix scanner (Twin II Flash; Philips Medical Systems (UK) Ltd, Stevenage, UK) and 
calibrated against a standard phantom. Images were acquired in $2.7 \mathrm{~mm}$ slices (with a $0.75 \mathrm{~s}$ full $360^{\circ} \mathrm{scan}$ mode) through the region of the coronary arteries with a pitch of 0.7 and an increment of $1.3 \mathrm{~mm}$ during held inspiration. Exposure factors were $120 \mathrm{kV}$ at $270 \mathrm{mAs}$ and the scan angle was $360^{\circ}$. Off-line analyses were conducted using an automated, computerised software program (Picker Cardiac Scoring). This employs an Agatston scoring method [Agatston et al 1990], producing sensitivity and specificity comparable to electron beam CT [Carr et al 2000]. Scans were scored using both the Agatston (130 HU threshold) and the modified Agatston (90 HU threshold) methods [Shemesh et al 1995]. The former has been shown to reduce interobserver and interscan variation compared to the threshold of $90 \mathrm{HU}$ [Goldin et al 2001]. To assess the reproducibility of the method, repeated baseline CT scans were performed within 4 weeks of each other in an unselected random sample of 16 patients.

\subsubsection{Data ANALYSIS AND STATISTICS}

Coronary artery calcium scores are expressed in AU using the $130 \mathrm{HU}$ threshold. The calcium scores and hsCRP concentrations were not normally distributed and data are presented as median with interquartile ranges or mean and standard deviation following logarithmic transformation. The primary end-point, the rate of change of coronary calcium scores, was analysed using random coefficient models [Bland and Altman 1986; Cowell et al 2005] after logarithmic transformation of the scores. In summarising the data, the change in coronary artery calcium scores was calculated by dividing the change between the baseline and final scores by the duration of follow-up. Rate of change in coronary calcium score is expressed as percentage change per year or as absolute change 
in the logarithm of the coronary artery calcium score. Reproducibility was assessed using the method of Bland and Altman [Bland and Altman 1986]. As well as tests of significance, $95 \%$ confidence intervals are reported as appropriate. Statistical significance was taken as a two-sided $\mathrm{p}$ value $<0.05$.

\subsection{RESULTS}

Of 155 patients recruited into the SALTIRE trial, 102 had coronary calcification at baseline (Figure 5.1) of whom 88 had at least two scans. Coronary calcification predominated in the left anterior descending coronary artery (100\% of patients) although it was also present in the circumflex (33\%) and right (27\%) coronary arteries. Baseline characteristics and coronary artery calcium scores were well matched in both treatment groups (Table 5.1) in the 88 evaluable subjects.

\subsubsection{REPRODUCIBILITY}

The reproducibility of the LAD coronary score and of the total coronary score was examined using the approach of Bland and Altman [Bland and Altman 1986]. Without transformation, the difference between replicate observations tended to increase with the magnitude of the measurement. After logarithmic transformation, higher values showed stable differences, but differences were higher at the lowest scores. Overall, the 


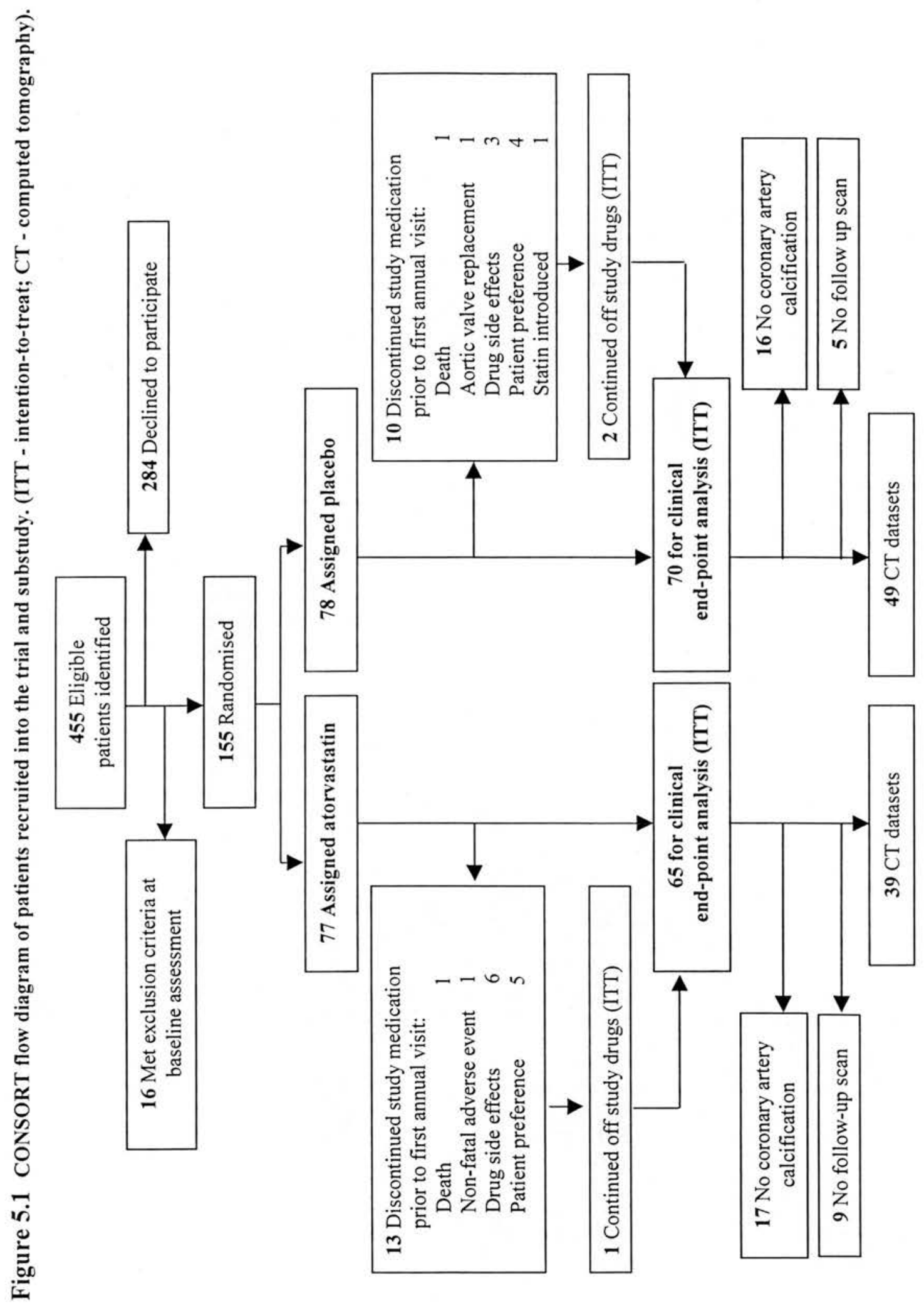




\begin{tabular}{|c|c|c|}
\hline & $\begin{array}{l}\text { Atorvastatin } \\
(\mathrm{n}=39)\end{array}$ & $\begin{array}{l}\text { Placebo } \\
(\mathrm{n}=49)\end{array}$ \\
\hline Age (years) & $70(8)$ & $70(9)$ \\
\hline Sex $(\%$ male $)$ & 74 & 78 \\
\hline Body mass index & $29(5)$ & $28(5)$ \\
\hline \multicolumn{3}{|l|}{ Cardiovascular Risk Factors } \\
\hline Hypertension & 22 & 28 \\
\hline Hyperlipidaemia & 3 & 2 \\
\hline Diabetes mellitus & 0 & 2 \\
\hline Current smoker & 5 & 10 \\
\hline \multicolumn{3}{|l|}{ Cardiovascular Disease } \\
\hline Coronary heart disease & 7 & 13 \\
\hline Cerebrovascular disease & 5 & 7 \\
\hline Peripheral vascular disease & 3 & 7 \\
\hline \multicolumn{3}{|l|}{ Drug History } \\
\hline Aspirin & 17 & 26 \\
\hline ACE inhibitor & 7 & 8 \\
\hline Beta-blocker & 11 & 15 \\
\hline Warfarin & 4 & 8 \\
\hline \multicolumn{3}{|l|}{ Blood Pressure (mmHg) } \\
\hline Systolic blood pressure & $143(18)$ & $140(19)$ \\
\hline Diastolic blood pressure & $82(11)$ & $78(11)$ \\
\hline \multicolumn{3}{|l|}{ Lipid Profile } \\
\hline Total cholesterol $(\mathrm{mmol} / \mathrm{L})$ & $5.7(0.9)$ & $5.5(0.9)$ \\
\hline LDL cholesterol (mmol/L) & $3.6(0.8)$ & $3.4(0.7)$ \\
\hline HDL cholesterol (mmol/L) & $1.5(0.4)$ & $1.5(0.4)$ \\
\hline Cholesterol: HDL ratio & $4.2(1.2)$ & $4.0(1.0)$ \\
\hline Triglycerides $(\mathrm{mmol} / \mathrm{L})$ & $1.5(0.8)$ & $1.4(0.7)$ \\
\hline \multicolumn{3}{|l|}{ Coronary calcification score (AU) } \\
\hline Left anterior descending & $112(40-285)$ & $207(76-461)$ \\
\hline Circumflex & $0(0-9)$ & $0(0-4)$ \\
\hline Right & $0(0-29)$ & $0(0-0)$ \\
\hline Total coronary score & $195(57-448)$ & $235(83-526)$ \\
\hline Log total coronary score ( $\log A U)$ & $2.16(0.68)$ & $2.30(0.65)$ \\
\hline
\end{tabular}

Continuous variables stated as mean (SD) or median (interquartile range).

Categorical variables stated as per cent.

ACE - angiotensin-converting enzyme; LDL - low-density lipoprotein; HDL - high-density lipoprotein; Log - logarithm. 
differences on the log scale correspond to a coefficient of variation of $28 \%$ for both variables, but when restricted to the ten pairs with a geometric mean score above 100 , the coefficient of variation was $10 \%$ for both variables.

\subsubsection{EFFECT OF ATORVASTATIN TREATMENT}

Patients were followed-up for a median of 24 months (interquartile range 24-30). Atorvastatin $80 \mathrm{mg}$ daily more than halved serum LDL cholesterol concentrations (53 (SD 19) \%; $\mathrm{p}<0.001$ ), whilst placebo had no effect (Figure 5.2). This reduction in serum LDL cholesterol concentrations was associated with a marked decrease in serum CRP concentrations from 1.95 (interquartile range $1.15-4.86)$ to $1.00(0.49-2.31) \mathrm{mg} / \mathrm{L}$ (Wilcoxon Signed Rank, $\mathrm{p}<0.001$; Figure 5.2). Atorvastatin was well tolerated with discontinuation of study medication in 2 patients on placebo and 5 patients on atorvastatin, predominantly as a result of gastrointestinal upset. One patient on atorvastatin had an increase in creatine kinase of $>5$ times the upper limit of normal without symptoms of myositis, and was withdrawn at the request of the Data Monitoring Committee. There were no cases of rhabdomyolysis.

\subsubsection{CORONARY ARTERY CALCIUM SCORE}

Atorvastatin did not affect the rate of progression of coronary artery calcium score (Figure 5.2). Similar results were obtained when employing the $90 \mathrm{HU}$ threshold (42 (SD 73) \%/yr in the atorvastatin group and 29 (SD 37) \%/yr in the placebo group; $\mathrm{p}=0.24$ ). Serum LDL cholesterol concentrations did not correlate with the rate of progression of coronary artery calcification $(r=0.05, \mathrm{p}=0.62)$. 
Figure 5.2 Progression of (A) coronary artery calcification, (B) serum C-reactive protein concentrations ( $\mathrm{p}<0.001$, atorvastatin versus placebo), and (C) serum low-density lipoprotein (LDL) cholesterol concentrations (p $s<0.001$, atorvastatin versus placebo) in patients treated with atorvastatin $80 \mathrm{mg}$ daily (solid circles) or matched placebo (open squares).

A

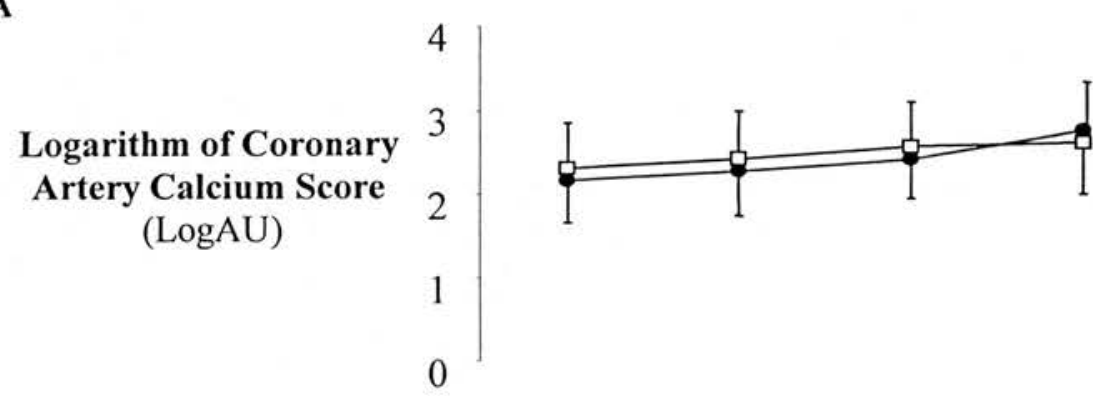

B

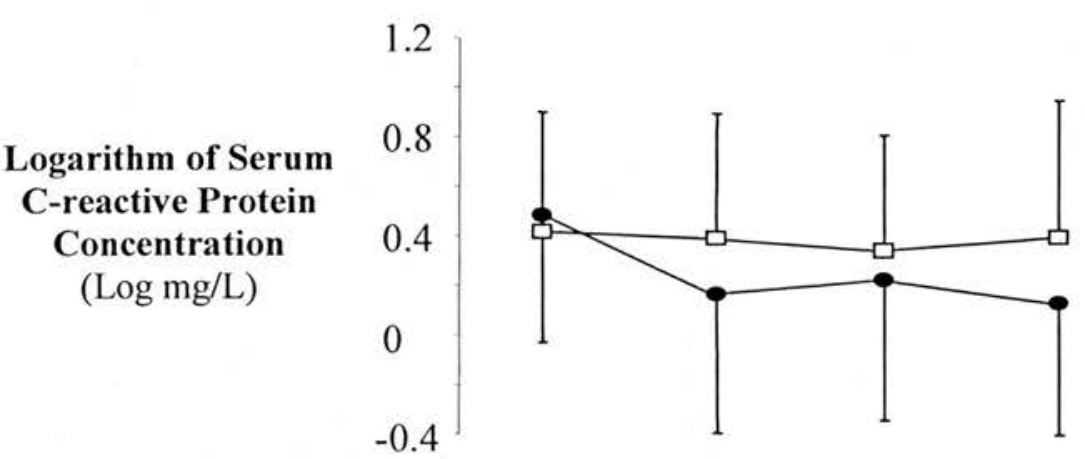

C

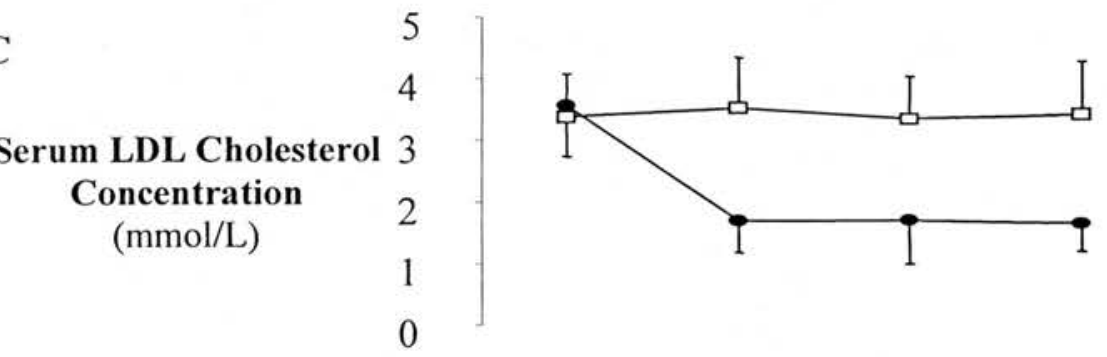

\begin{tabular}{|c|c|c|c|c|}
\hline$\rightarrow$ & Atorvastatin & 39 & 39 & 36 \\
\hline & Placebo & 49 & 49 & 40 \\
\hline & & $r$ & 1 & $T$ \\
\hline & & 0 & 12 & 24 \\
\hline
\end{tabular}

LDL - low-density lipoprotein; Log - logarithm; AU - arbitrary units. 
The primary analysis of the rates of change of coronary artery calcium scores was conducted on the logarithms of the scores using random coefficients models [Brown and Prescott 1999]. This showed no difference between the average rates of change in the two treatment arms $(\mathrm{p}=0.18)$. The mean coronary calcium score increased by 0.234 (SE 0.037) $\log \mathrm{AU}$ per year in the atorvastatin group and 0.167 (SE 0.034) log AU per year in the placebo group. These figures correspond to a $26 \%$ per year increase in the atorvastatin group and $18 \%$ per year in the placebo group. The geometric mean (adjusted for baseline) is $7 \%$ higher at one year on atorvastatin compared to placebo, with $95 \%$ confidence limits ranging from $3 \%$ lower to $18 \%$ higher. The observed annual changes in coronary calcium scores, calculated from the first to the last visit, are summarised in Figure 5.3.

As anticipated in such a small clinical trial, there were no significant differences in all cause mortality, cardiovascular mortality or cardiovascular hospitalisation between the two groups. 

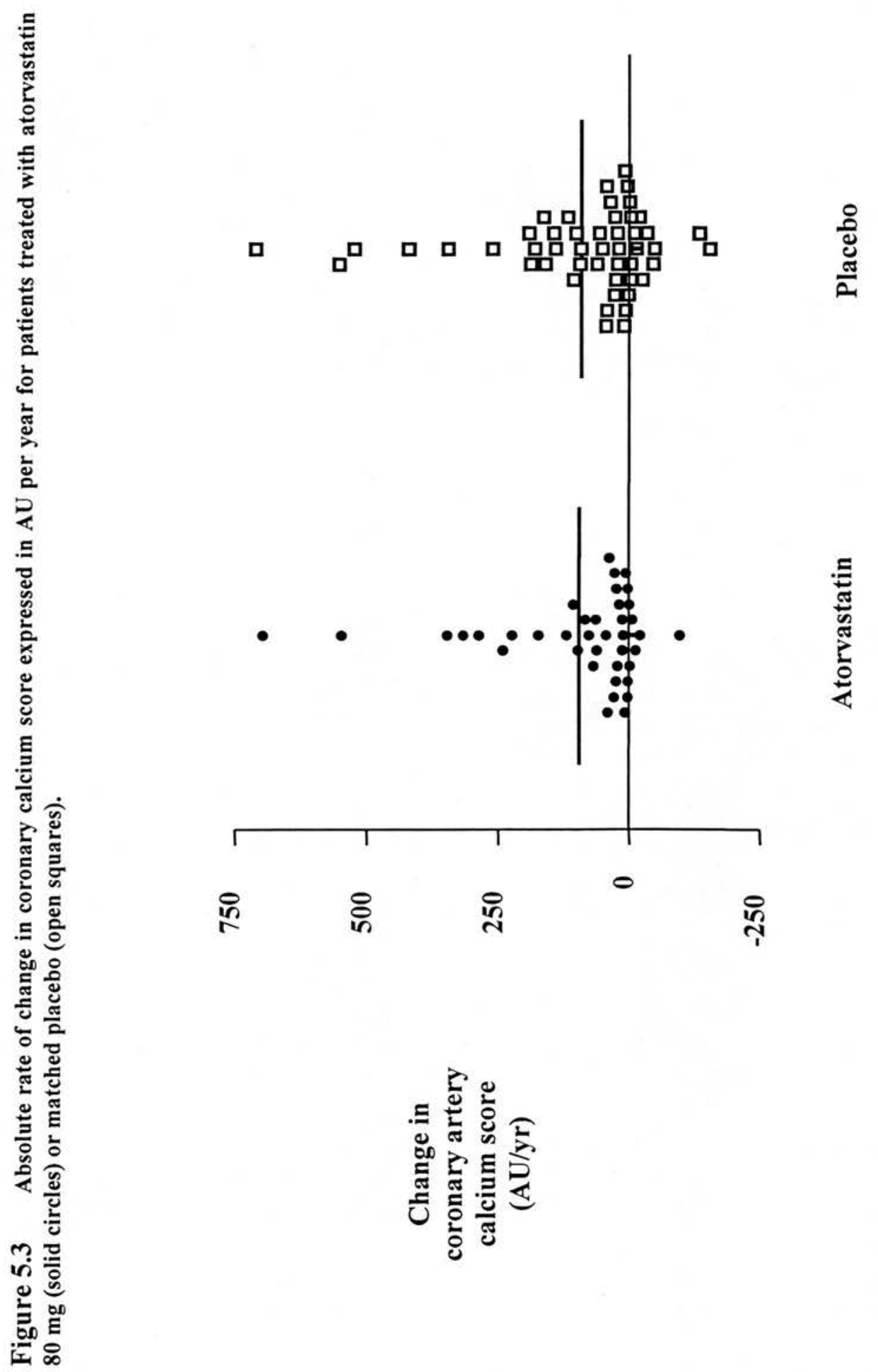

育 


\subsection{DISCUSSION}

We have confirmed that, despite marked reductions in serum LDL cholesterol and CRP concentrations, atorvastatin $80 \mathrm{mg}$ daily did not halt the progression, or induce regression, of coronary artery calcification in patients with calcific aortic stenosis. Consistent with recent trials of asymptomatic individuals [Arad et al 2005; Raggi et al 2005], our findings are in marked contrast to previous observational studies and suggest that the potential beneficial effects of statin therapy on coronary artery calcification have been over estimated.

Previous observational and non-randomised prospective studies [Callister et al 1998; Achenbach et al 2002] have suggested that reductions in serum LDL cholesterol concentrations decrease the progression of coronary calcification. However, not all observational studies have demonstrated consistent findings. In the largest observational study of 182 patients, Hecht and colleagues recently found no difference in the progression of coronary calcium scores in patients who were maintained on lipidlowering therapy and achieved significant reductions in serum LDL cholesterol concentrations [Hecht and Harman 2003]. Observational data may be misleading and prospective randomised controlled trials are necessary to confirm or to refute these interesting preliminary observations. The recent BELLES trial [Raggi et al 2005] found no differential effect of pravastatin (40 mg daily) and atorvstatin ( $80 \mathrm{mg}$ daily) on the progression of coronary artery calcification in 615 hyperlipidaemic post-menopausal women. However, study follow-up was brief (1 year) and there was no placebo control 
group. The St Francis Heart Study [Arad et al 2005] randomised 1,005 asymptomatic middle-aged men and women with high coronary artery calcium scores to combination atorvastatin $20 \mathrm{mg}$, vitamin C $1 \mathrm{~g}$ and vitamin $\mathrm{E}$ (alpha tocopherol) 1,000 units daily or matching placebos. After 4.3 years of follow-up, there were no differences in the rate of progression of coronary artery calcification.

We have conducted a double-blind randomised controlled trial using helical CT in patients with aortic stenosis. Minimisation technique ensured good matching of the baseline characteristics of the patient population and reproducibility studies confirmed the validity of our repeated assessments. Although documenting very similar rates of progression of coronary calcification to previous studies [Callister et al 1998; Achenbach et al 2002; Hecht and Harman 2003], we have not observed a reduction in coronary calcification with intensive lipid-lowering therapy despite more than halving serum LDL cholesterol concentrations.

Statin therapy has been extremely successful in the primary and secondary prevention of cardiovascular disease. Why then have we and others not observed a beneficial effect of statin therapy on coronary artery calcification? Unstable atherosclerotic plaques have a large lipid-rich core, a preponderance of macrophages and foam cells, and a thin fibrous cap containing few smooth muscle cells [Davies 1997]. It has been suggested that calcified lesions may be relatively more stable [Mintz et al 1995], indicating a possible protective role of calcification in coronary plaques. Statin therapy produces many of its beneficial effects through plaque stabilisation. In both primate [Stary 2001] and swine 
[Daoud et al 1981] models, anti-atherosclerotic interventions are associated with an increase in vascular fibrous tissue and calcification. This calcium deposition continues during the initial phase of plaque regression due to the death of foam cells and an increase in necrotic tissue. Thus vascular calcification may play a role in the initial stabilisation of atherosclerotic plaques. This is consistent with our findings and would account for the lack of effect on the progression of coronary artery calcification despite a reduction in serum CRP concentrations.

After the initial stabilisation of the atherosclerotic plaque, it would be anticipated that subsequent progression of coronary calcification would be inhibited. The present study was brief, and follow-up was only continued for a median of 2 years. It would be important to extend our observations to 5 or more years to assess properly the impact of statin therapy on the long-term progression of coronary artery calcification. However, it should be acknowledged that the clinical benefits of statin therapy are apparent within the first few years [Lewis et al 1998; The LIPID Study Group 1998; The Heart Protection Study Collaborative Group 2002], and in some cases the first few months [Schwartz et al 1998], of therapy. Moreover, the St Francis Heart Study demonstrated no beneficial effects despite 4.3 years of follow-up [Arad et al 2005].

On the basis of previous non-randomised studies [Achenbach et al 2002], the practice of performing serial CT scans to monitor disease progression and the response to treatment has become widespread, especially in North America. Our data, and that of the St Francis Heart Study [Arad et al 2005] and the BELLES study [Raggi et al 2005], 
indicate that repeated scanning to assess response to statin therapy is not justified. Indeed, the radiation dose incurred for such serial scans poses potential health risks, particularly when employing multidetector CT scanners.

\subsubsection{STUDY LIMITATIONS}

There are several factors that should be taken into account when considering the results of our study. This was a substudy of the SALTIRE trial [Cowell et al 2005] that recruited only patients with calcific aortic stenosis. However, our findings are consistent with two recent randomised controlled trials in asymptomatic younger individuals without valvular heart disease [Arad et al 2005; Raggi et al 2005]. Our study therefore suggests that failure of statins to restrict the progression of coronary artery calcification can be extended to include patients with valvular heart disease as well as more elderly populations. Moreover, our findings suggest that lack of benefit seen in the St Francis Heart Study is not attributable to the modifying effects of antioxidant vitamins [Arad et al 2005].

When compared with electron beam CT, the accuracy of helical CT in detecting coronary artery calcification has been questioned [Carr et al 2000; Qanadli et al 2001]. Technological advances have also meant that double-helical scanners have now been overtaken by 64 or higher slice scanners. At trial inception, the double-helix scanner was "state-of-the-art" and it would have been inappropriate to replace the scanner during the conduct of the trial. Moreover, our approach has been previously validated [Bland and Altman 1986] and we have demonstrated good reproducibility of coronary artery 
calcification scores in patients with scores of greater than $100 \mathrm{AU}$. We do not believe the absence of a major beneficial effect on coronary artery calcification is attributable to our methodology. We acknowledge the fact that our population size is modest; however, the $95 \%$ confidence intervals are able to exclude a relative reduction in progression of coronary artery calcification of $>3 \%$ per year. We therefore suggest that if lipid-lowering therapy does reduce the progression of coronary artery calcification then the effect is rather small.

Controversy exists over the method of quantification of coronary artery calcification. The Agatston method is traditionally employed but this may overestimate the coronary calcium score in newer generation scanners with reduced slice thickness due to partial voluming. More recent methods include the volume [Callister et al 1998] and the coronary calcium mass [Hong et al 2002] scores, although neither are superior to the Agatston score in terms of reproducibility from consecutive scans in an individual patient [Rumberger and Kaufman 2003].

\subsection{CONCLUSION}

We conclude that intensive lipid-lowering therapy does not halt the progression, or induce regression, of coronary artery calcification. Although coronary artery calcium scores correlate well with the presence of atherosclerosis and predict future coronary risk, our findings confirm that there is currently no role for monitoring progression of coronary artery calcification in order to assess the response to lipid-lowering therapy. 


\section{CHAPTER 6}

PREDICTORS OF DISEASE PROGRESSION AND

CLINICAL OUTCOME IN PATIENTS WITH

CALCIFIC AORTIC STENOSIS 


\subsection{SUMMARY}

Background Calcific aortic stenosis results from an active inflammatory process closely resembling atherosclerosis. We hypothesised that risk factors for atherosclerosis would predict disease progression and clinical outcome of patients with calcific aortic stenosis.

Methods Patients $(\mathrm{n}=155 ; 68 \pm 11 \mathrm{yrs})$ with calcific aortic stenosis participating in the SALTIRE trial (randomised comparison of atorvastatin $80 \mathrm{mg}$ daily or matched placebo) were followed-up for $26 \pm 7$ months. Severity of aortic valve stenosis and calcification was assessed longitudinally using echocardiography and helical CT respectively.

Results Aortic jet velocity progressed by $0.20 \pm 0.21 \mathrm{~m} / \mathrm{s}$ per year and calcification by $29 \pm 30 \%$ per year and was unaffected by atorvastatin therapy. Disease progression was predicted by age $(p<0.01)$, sex $(p=0.02)$, height $(p=0.03)$, hypertension $(\mathrm{p}=0.03)$, serum BNP concentration $(\mathrm{p}=0.002)$, and baseline valve disease severity as determined by both aortic jet velocity $(\mathrm{p}<0.001)$ and valvular calcification $(\mathrm{p}<0.001)$. Clinical outcome was predicted by baseline and rate of progression of aortic stenosis severity $(\mathrm{p}<0.002)$ and serum BNP concentrations $(\mathrm{p} \leq 0.02)$. Clinical and biochemical markers of atherosclerosis, including serum CRP and cholesterol concentrations, did not predict disease progression or clinical outcome.

Conclusions The major predictors of disease progression and clinical outcome in patients with aortic stenosis are measures of disease severity; namely aortic jet velocity, aortic valve calcification and serum BNP concentration. With the exception 
of hypertension the presence of clinical atherosclerotic risk factors do not influence the rate of progression.

\subsection{INTRODUCTION}

Aortic stenosis is the commonest adult heart valve condition seen in the western world affecting $2 \%$ of individuals over the age of 65 [Cowell et al 2004]. Thickening and calcification of the valve leaflets progresses slowly over many years. The outlook for patients with asymptomatic aortic stenosis is generally good, but although prognosis is similar to life table estimates for age and sex matched controls [Pellikka et al 1990], there is an increased risk of unrelated cardiovascular events [Iivanainen et al 1996; Otto et al 1999; Rosenhek et al 2004]. In patients with severe stenosis, the onset of symptoms dramatically changes the outlook with 2-year survival rates falling to $50 \%$ [Otto et al 1999; Rosenhek et al 2000]. At the present time, the only treatment option of prognostic benefit is aortic valve replacement.

Calcific aortic stenosis has for many decades been attributed to age-associated "wear and tear", but a third of individuals over the age of 80 [Lindroos et al 1993] have no evidence of aortic valve calcification or stenosis. Moreover, histological examination of stenotic aortic valves reveals features of chronic inflammation that resemble those seen in atherosclerosis, and are distinct from those changes occurring with ageing alone [Olsson et al 1994; Otto et al 1994]. In addition, calcific aortic stenosis is associated with coronary artery disease and its risk factors including age, male sex, 
smoking, hypertension, diabetes mellitus, coronary artery disease and hyperlipidaemia [Cowell et al 2004].

Disease progression in aortic stenosis is variable and unpredictable. Both disease progression and clinical outcome have been linked to many of the risk factors for aortic stenosis, but much of the evidence is conflicting and based on retrospective studies [Peter et al 1993; Bahler et al 1999; Palta et al 2000; Ngo et al 2001; Wongpraparut et al 2002]. The most consistent and strongest predictors of disease progression appear to be severity of stenosis [Otto et al 1997; Bahler et al 1999] and the degree of valvular calcification [Davies et al 1991; Bahler et al 1999; Rosenhek et al 2004a]. The more severe the stenosis and the more heavily calcified the valve, the faster the rate of disease progression. Clinical outcome is influenced by the rate of disease progression as well as the degree of valvular calcification, with nearly $80 \%$ of patients with moderate to severe calcification who progress rapidly $(>0.3$ $\mathrm{m} / \mathrm{s} / \mathrm{yr}$ ) either dying or undergoing AVR within 2 years [Rosenhek et al 2000].

Using the SALTIRE trial population [Cowell et al 2005], we wished to identify prospectively the clinical and biochemical variables associated with disease progression and clinical outcome in patients with calcific aortic stenosis. We assessed clinical risk factors as well as other novel predictors of cardiovascular risk including inflammatory, vascular and cardiac markers. In particular, we hoped to identify modifiable risk factors, with the potential of delaying or avoiding the need for AVR. Identification of markers capable of predicting risk might also facilitate the appropriate timing of aortic valve surgery. 


\subsection{METHODS}

\subsubsection{Patient population}

All 155 patients randomised to the SALTIRE trial (a randomised controlled trial assessing the effects of atorvastatin therapy on the progression of aortic valve stenosis and calcification) [Cowell et al 2005] were included in this substudy. Patients aged $>18$ years with calcific aortic stenosis, an aortic jet velocity of $\geq 2.5 \mathrm{~m} / \mathrm{s}$, and grade 1-3 calcification of the aortic valve on echocardiography were included. Exclusion criteria are detailed in Chapter 2. All patients had asymptomatic aortic stenosis at entry into the study, and were randomised to receive either atorvastatin $80 \mathrm{mg}$ daily or matched placebo. The investigation conformed to the Declaration of Helsinki, and was approved by all regional ethics committees. All patients gave written informed consent.

\subsubsection{STUDY PROTOCOL}

Patients were assessed at baseline, at 2 and 6 months, and 6 monthly thereafter for a minimum of 2 years. Baseline characteristics evaluated included demographics, cardiovascular risk factors, functional status (NYHA classification), blood pressure, arterial stiffness (pulse wave analysis), and biochemical variables including lipid profile, renal function, BNP, CRP and calcium concentrations. Subsequent evaluation comprised assessment of biochemical blood analysis, arterial stiffness and adverse clinical events. Echocardiography and CT were performed at baseline, at each annual visit or prior to withdrawal from the study. 


\subsubsection{ECHOCARDIOGRAPHY}

Assessment of valvular stenosis was determined by a single dedicated research ultrasonographer. Patients were studied using a 3-MHz transducer for M-mode, and two-dimensional imaging with integral pulsed, continuous wave and tissue Doppler. All measurements were determined on-line, averaged from three cardiac cycles (five cycles if in atrial fibrillation), and recorded onto Super-VHS video and optical disk using a standardised proforma. Peak and mean aortic valve gradients were calculated using the Bernoulli equation, and AVA using the continuity equation.

\subsubsection{COMPUTED TOMOGRAPHY}

Computed tomography was performed by a single operator using a double-helix scanner (Twin II Flash; Philips Medical Systems Ltd, Stevenage, UK) and calibrated against a standard phantom. The region of the aortic valve was imaged with a spiral acquisition using $2.7 \mathrm{~mm}$ slices, with a pitch of 0.7 and an increment of $1.4 \mathrm{~mm}$ during held inspiration. All images were analysed by a single operator using automated computerised software (Picker Cardiac Scoring). This employs a modified Agatston scoring method [Shemesh et al 1995] that uses a threshold of $90 \mathrm{HU}$ to compensate for non-gated imaging.

Reproducibility of echocardiography and CT assessments was determined in two subsets of 20 patients as described in Chapter 3. Coefficients of reproducibility for aortic jet velocity and AVC score were $0.32 \mathrm{~m} / \mathrm{s}$ and $0.07 \mathrm{pAU}$ respectively. 


\subsubsection{BIOCHEMICAL VARIABLES}

Fasting venous blood samples were taken annually. Samples for serum electrolytes, lipid profile and calcium concentrations were sent to the regional clinical laboratory for immediate analysis. Samples for estimation of serum BNP and CRP concentrations were centrifuged at $4^{\circ} \mathrm{C}$ and stored at $-80^{\circ} \mathrm{C}$ for later analysis. $\mathrm{N}$ terminal pro-BNP was measured using a chemiluminescent immunoassay (Roche Diagnostics Ltd, Lewes, U.K.) on an Elecsys 2010 analyser. Serum highly sensitive CRP concentrations were determined using a highly sensitive immunonephelometric method (Dade Behring Ltd, Milton Keynes, UK).

\subsubsection{Pulse wave analysis}

Pulse wave analysis was performed at baseline and 6 monthly intervals thereafter. Patients were rested supine for 15 minutes prior to study. Radial pulse wave analysis was performed using a high-fidelity applanation tonometer (Sphygmocor BPAS; PWV Medical, Sydney, Australia). After acquiring a series of consecutive waveforms, an averaged peripheral waveform was acquired and a generalised transfer function was used to generate a central aortic pressure waveform from which the pulse pressure, augmentation pressure and augmentation index were determined. Two measurements of augmentation within $5 \mathrm{mmHg}$ of each other were recorded on each occasion. Patients with atrial fibrillation were excluded, and recordings with poor quality waveforms were discarded: determined by visual inspection (SJC) with minimum requirements in pulse height of $>100 \mathrm{mmHg}$, diastolic variation of $<5 \%$ and pulse height variation of $<5 \%$. Data on 105 patients were available at baseline, of these 20 patients had poor quality recordings and 9 patients were in atrial fibrillation, leaving a total of 84 patients for analysis. 


\subsubsection{Clinical Follow-UP}

The following clinical end-points were recorded throughout the study; cardiovascular and all cause mortality, AVR (whether for severe symptomatic stenosis or not), and the development of symptoms attributable to severe aortic stenosis (confirmed by the patient's treating physician).

\subsubsection{DATA ANALYSIS AND STATISTICS}

Disease progression was determined by measuring the annual rate of change in aortic jet velocity on echocardiography, and the change in aortic valve calcification on CT by dividing the difference between baseline and final scans by the duration of followup. The CT, BNP and CRP data were log transformed prior to analysis as the data were not normally distributed. Predictors of progression were determined using regression analysis for continuous variables, and Chi-squared tests for categorical variables. Predictors of outcome were determined using Chi-squared tests for categorical variables, $t$-tests for continuous variables. Statistical significance was taken as two-sided $\mathrm{p}<0.05$.

\subsection{RESULTS}

One hundred and fifty-five patients (mean age 68 years, $70 \%$ male) with calcific aortic stenosis were randomised to the SALTIRE trial [Cowell et al 2005] and were followed-up for a median of 25 months (range 7-36). 
At baseline (Table 6.1) mean aortic jet velocity was $3.42 \pm 0.64 \mathrm{~m} / \mathrm{s}$ (range 2.5-5.0), and increased by $0.20 \pm 0.21 \mathrm{~m} / \mathrm{s}$ per year (Table 6.2). Median AVC score at baseline was $5920 \mathrm{AU}$ (interquartile range $2485-14231 \mathrm{AU}$ ), and increased by $29 \pm 30 \%$ per year. A number of patients had risk factors for cardiovascular disease (Table 6.1) and at baseline the mean total cholesterol was $5.7 \mathrm{mmol} / \mathrm{L}$. As previously reported (Chapter 4) [Cowell et al, 2005], there was no difference between the treatment groups despite a $53 \%$ reduction in serum LDL cholesterol concentrations $(\mathrm{p}<0.001)$ in the atorvastatin group. Given the absence of a treatment effect, all patients have been included in the subsequent analyses.

\subsubsection{DISEASE PROGRESSION}

The results of univariate analyses are presented in Tables 6.3 and 6.4. In keeping with previous observation studies [Bahler et al 1999], we have now demonstrated prospectively that progression of aortic jet velocity is strongly correlated with baseline aortic jet velocity $(\mathrm{p}<0.001$; Table 6.3 ), and furthermore is correlated with baseline and progressive increases in the absolute AVC score $(\mathrm{p}=0.004$ and $\mathrm{p}=0.002$ respectively). Annual progression in aortic jet velocity was also strongly associated with age $(p=0.006)$, baseline log $B N P(p=0.003)$ and the rate of change in log BNP per year $(p=0.002)$, but only weakly with height $(p=0.03)$, male sex $(p=0.02)$ and the presence of hypertension $(\mathrm{p}=0.03)$ (Tables 6.3 and 6.4). 
All patients

$\mathrm{n}=\mathbf{1 5 5}$

\section{Demographics}

Age (years)

$67.8(10.6)$

Male Sex

$108(69.7 \%)$

Cardiovascular Risk Factors

Hypertension

$79(50.6 \%)$

Hypercholesterolaemia

$10(6.5 \%)$

Diabetes Mellitus

$5(3.2 \%)$

Current smoker

$33(21.3 \%)$

Cardiovascular Disease

Coronary artery disease

$30(19.4 \%)$

Cerebrovascular disease

$16(10.3 \%)$

Peripheral vascular disease

$14(9.0 \%)$

Drug History

Aspirin

$64(41.0 \%)$

Warfarin

$15(9.6 \%)$

Beta-blocker

$37(23.7 \%)$

ACE inhibitor

$20(12.8 \%)$

NYHA Class

Class I

$12(8 \%)$

Class II

$125(80.6 \%)$

Class III

$18(11.6 \%)$

Class IV

0

\section{Physical Examination}

Height $(\mathrm{cm})$

$168.5(8.3)$

Weight $(\mathrm{Kg})$

$79.3(14.8)$

Heart rate (beats/min)

67 (11.6)

Systolic blood pressure $(\mathrm{mmHg})$

Diastolic blood pressure $(\mathrm{mmHg})$

\section{Electrocardiography}

Sinus rhythm

Atrial fibrillation

\section{Echocardiography}

Bicuspid valve

Tricuspid valve 
TABLE 6.1 Baseline Characteristics continued /

Echocardiography

Peak aortic jet velocity $(\mathrm{m} / \mathrm{s})$

Peak gradient $(\mathrm{mmHg})$

Aortic valve area $\left(\mathrm{cm}^{2}\right)$

LV mass (g)

$\mathrm{LV}$ mass index $\left(\mathrm{g} / \mathrm{m}^{2}\right)$

Fractional shortening (\%)

Computerised Tomography

AV calcium score (AU) $\dagger$

Mean Log AV calcium score (LogAU)

Pulse Wave Analysis

Pulse pressure, $\mathrm{PP}(\mathrm{mmHg})$

Augmentation pressure, $\mathrm{AG}(\mathrm{mmHg})$

Augmentation index AG/PP (\%)

Augmentation index P2/P1 (\%)

\section{Serum Biochemistry}

Total cholesterol $(\mathrm{mmol} / \mathrm{L})$

HDL cholesterol ( $\mathrm{mmol} / \mathrm{L})$

HDL:Total cholesterol ratio

Triglycerides $(\mathrm{mmol} / \mathrm{L})$

LDL cholesterol $(\mathrm{mmol} / \mathrm{L})$

Urea $(\mathrm{mmol} / \mathrm{L})$

Creatinine $(\mu \mathrm{mol} / \mathrm{L})$

Glucose $(\mathrm{mmol} / \mathrm{L})$

Calcium $(\mathrm{mmol} / \mathrm{L})$

Brain Natriuretic Peptide (pg/ml)

Mean (SD)

Median (Range)

Log BNP (Log pg/ml)

\section{C-Reactive Protein (mg/L)}

Mean (SD)

Median (Range)

Log CRP (Log mg/L) $\mathbf{n}=\mathbf{1 5 5}$

$3.42(0.64)$

48.6 (18.4)

$1.02(0.4)$

$354(110)$

$176(55)$

$40(8.4)$

$\mathrm{n}=\mathbf{1 5 5}$

$5920(2485-14231)$

$3.67(0.53)$

$\mathrm{n}=110$

61 (17)

$16(9)$

$31(12)$

$149(29)$

$\mathbf{n}=\mathbf{1 5 5}$

$5.7(1.0)$

$1.5(0.4)$

$4.1(1.2)$

$1.5(0.7)$

$3.5(0.8)$

$6.5(5.0)$

$92(21.4)$

$5.3(1.2)$

$2.32(0.10)$

$\mathrm{n}=\mathbf{1 3 4}$

465 (633)

200 (14-3640)

$2.37(0.52)$

$\mathbf{n}=\mathbf{1 3 5}$

$4.3(6.2)$

$2.35(0.16-38.0)$

$0.39(0.5)$

Categorical variables stated as number, $\mathrm{n}(\%)$. Continuous variables stated as mean (standard deviation). $\dagger$ Median (Range).

ACE - angiotensin-converting enzyme; NYHA - New York Heart Association; LV - left ventricular; AV - aortic valve; Log - logarithm; HDL - high-density lipoprotein; LDL - low density lipoprotein; BNP - brain natriuretic peptide. 
TABLE 6.2 Progression of aortic valve stenosis and calcification

$\begin{aligned} & \text { Progression of stenosis } \\ & \text { (Echocardiography) }\end{aligned} \quad \mathbf{n}=134$

Peak velocity $(\mathrm{m} / \mathrm{s} / \mathrm{yr})$

$0.20(0.21)$

Peak gradient $(\mathrm{mmHg} / \mathrm{yr})$

Aortic valve area $\left(\mathrm{cm}^{2} / \mathrm{yr}\right)$

$-0.08(0.11)$

Progression of calcification

$\mathbf{n}=\mathbf{1 3 3}$

(Computed Tomography)

$1608(1865)$

Absolute change AVC score (AU/yr)

$29(30)$

Per cent change absolute AVC score (\%/yr)

Log AVC score (LogAU)

$0.09(0.10)$

Variables stated as mean (standard deviation).

AVC - aortic valve calcium; Log - logarithm. 
TABLE 6.3 Predictors of Disease Progression

\begin{tabular}{|c|c|c|c|c|c|c|c|c|}
\hline & \multicolumn{2}{|c|}{$\begin{array}{c}\text { Change in } \\
\text { peak velocity } \\
(\mathrm{m} / \mathrm{s} / \mathrm{yr})\end{array}$} & \multicolumn{2}{|c|}{$\begin{array}{l}\text { Change in } \\
\text { absolute } \\
\text { AVC score } \\
\text { (AU/yr) }\end{array}$} & \multicolumn{2}{|c|}{$\begin{array}{c}\text { Change in } \\
\text { Log } \\
\text { AVC score } \\
\text { (LogAU/yr) }\end{array}$} & \multicolumn{2}{|c|}{$\begin{array}{c}\text { Per cent } \\
\text { change in AVC } \\
\text { score } \\
(\% / y r) \\
\end{array}$} \\
\hline & $\mathbf{R}$ & p & $\mathbf{R}$ & p & $\mathbf{R}$ & $\mathbf{p}$ & $\mathbf{R}$ & $\mathbf{P}$ \\
\hline \multicolumn{9}{|l|}{ Baseline demographics } \\
\hline Age (years) & +0.24 & 0.006 & +0.21 & 0.02 & -0.10 & 0.24 & -0.03 & 0.71 \\
\hline Height & -0.19 & 0.03 & +0.23 & 0.01 & -0.15 & 0.09 & -0.12 & 0.19 \\
\hline Weight & -0.13 & 0.15 & +0.12 & 0.18 & +0.05 & 0.56 & +0.17 & 0.06 \\
\hline \multicolumn{9}{|l|}{ Baseline disease severity } \\
\hline Peak velocity & +0.32 & $<0.001$ & +0.48 & $<0.001$ & -0.05 & 0.60 & -0.02 & 0.80 \\
\hline Absolute AVC score & +0.25 & 0.004 & +0.49 & $<0.001$ & -0.26 & 0.003 & -0.30 & $<0.001$ \\
\hline $\log$ AVC score & +0.26 & 0.003 & +0.47 & $<0.001$ & -0.32 & $<0.001$ & -0.40 & $<0.001$ \\
\hline \multicolumn{9}{|l|}{ Disease progression } \\
\hline Change peak velocity/yr & & & +0.27 & 0.002 & -0.02 & 0.83 & +0.05 & 0.58 \\
\hline Change absolute AVC/yr & +0.27 & 0.002 & & & +0.2 & 0.02 & +0.27 & 0.002 \\
\hline Change log AVC/yr & -0.01 & 0.88 & +0.20 & 0.02 & & & +0.94 & $<0.001$ \\
\hline Per cent change $\mathrm{AVC} / \mathrm{yr}$ & +0.05 & 0.54 & +0.27 & 0.002 & +0.94 & $<0.001$ & & \\
\hline \multicolumn{9}{|l|}{ Blood pressure $(\mathrm{mmHg})$} \\
\hline Baseline systolic BP & -0.04 & 0.68 & -0.01 & 0.94 & +0.14 & 0.11 & +0.19 & 0.03 \\
\hline Baseline diastolic BP & +0.04 & 0.67 & +0.05 & 0.59 & +0.13 & 0.15 & +0.13 & 0.15 \\
\hline Mean study systolic BP & $<-0.01$ & 0.98 & -0.01 & 0.92 & +0.06 & 0.48 & +0.18 & 0.04 \\
\hline Mean study diastolic BP & +0.11 & 0.22 & +0.03 & 0.71 & +0.06 & 0.46 & +0.11 & 0.20 \\
\hline \multicolumn{9}{|l|}{ Baseline serum biochemistry } \\
\hline Urea $(\mathrm{mmol} / \mathrm{L})$ & -0.12 & 0.15 & -0.07 & 0.41 & +0.08 & 0.33 & +0.20 & 0.02 \\
\hline Creatinine $(\mu \mathrm{mol} / \mathrm{L})$ & $<-0.01$ & 0.95 & -0.04 & 0.62 & -0.06 & 0.49 & -0.07 & 0.39 \\
\hline Calcium $(\mathrm{mmol} / \mathrm{L})$ & +0.02 & 0.86 & -0.12 & 0.17 & -0.03 & 0.77 & +0.21 & 0.02 \\
\hline Total cholesterol $(\mathrm{mmol} / \mathrm{L})$ & +0.14 & 0.10 & -0.11 & 0.22 & -0.03 & 0.75 & -0.07 & 0.40 \\
\hline HDL cholesterol (mmol/L) & +0.16 & 0.06 & +0.12 & 0.17 & +0.17 & 0.05 & +0.16 & 0.06 \\
\hline LDL cholesterol ( $\mathrm{mmol} / \mathrm{L})$ & +0.11 & 0.21 & -0.10 & 0.27 & -0.06 & 0.51 & -0.11 & 0.20 \\
\hline \multicolumn{9}{|l|}{$\begin{array}{l}\text { Mean on treatment serum } \\
\text { biochemistry }\end{array}$} \\
\hline Urea $(\mathrm{mmol} / \mathrm{L})$ & -0.05 & 0.59 & -0.02 & 0.79 & -0.09 & 0.33 & +0.06 & 0.48 \\
\hline Creatinine $(\mu \mathrm{mol} / \mathrm{L})$ & -0.14 & 0.10 & $<-0.01$ & 0.99 & -0.08 & 0.38 & -0.05 & 0.58 \\
\hline Calcium $(\mathrm{mmol} / \mathrm{L})$ & +0.11 & 0.22 & -0.10 & 0.25 & +0.20 & 0.02 & +0.24 & 0.007 \\
\hline Total cholesterol (mmol/L) & +0.05 & 0.58 & -0.02 & 0.79 & -0.09 & 0.33 & -0.10 & 0.24 \\
\hline HDL cholesterol (mmol/L) & +0.14 & 0.11 & +0.14 & 0.10 & +0.14 & 0.10 & +0.14 & 0.10 \\
\hline LDL cholesterol (mmol/L) & +0.03 & 0.70 & -0.03 & 0.71 & -0.11 & 0.23 & -0.12 & 0.17 \\
\hline \multicolumn{9}{|l|}{ C-reactive protein } \\
\hline Log baseline CRP & -0.14 & 0.11 & -0.13 & 0.12 & -0.17 & 0.05 & -0.15 & 0.09 \\
\hline Log mean study CRP & -0.10 & 0.25 & -0.04 & 0.62 & -0.15 & 0.08 & -0.13 & 0.15 \\
\hline Change log CRP/year & +0.07 & 0.42 & +0.01 & 0.91 & -0.04 & 0.68 & +0.03 & 0.75 \\
\hline \multicolumn{9}{|l|}{ Brain natriuretic peptide } \\
\hline Log baseline BNP & +0.26 & 0.003 & +0.11 & 0.21 & -0.17 & 0.06 & -0.08 & 0.36 \\
\hline Change log $\mathrm{BNP} /$ year & +0.26 & 0.002 & +0.32 & $<0.001$ & -0.05 & 0.61 & +0.05 & 0.57 \\
\hline
\end{tabular}

AVC - aortic valve calcium; Log - logarithm; BP - blood pressure; HDL - high-density lipoprotein; LDL - low-density lipoprotein; CRP - C-reactive protein; BNP - brain natriuretic peptide. 


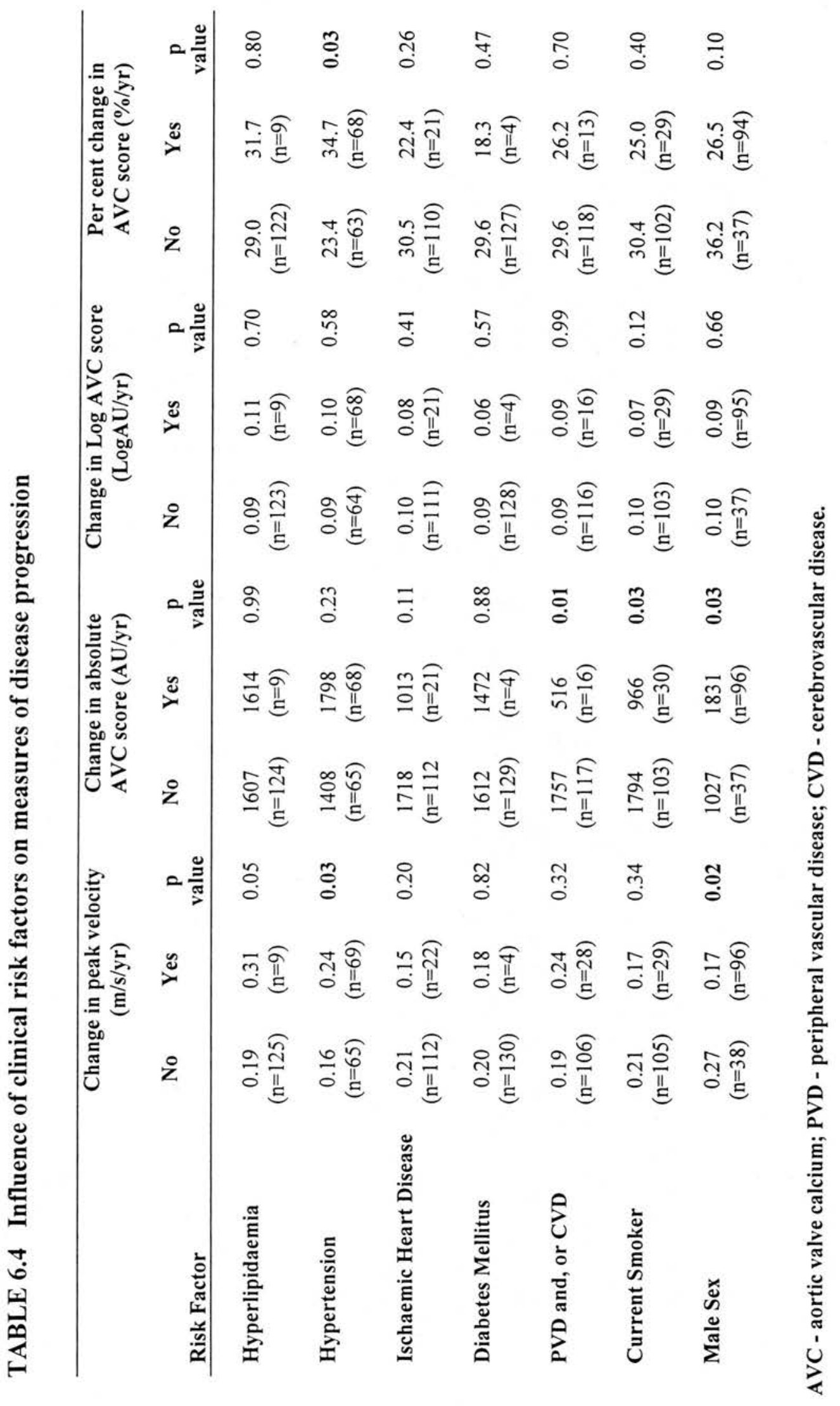


Progression of valvular calcification was predicted by the baseline AVC score $(p<0.001)$ and the rate of change in log BNP per year $(p<0.001)$. Age $(p=0.02)$, height $(p=0.009)$, mean study systolic blood pressure $(p=0.04)$ and serum calcium concentrations $(\mathrm{p}=0.02)$ and the presence of hypertension $(\mathrm{p}=0.03)$ also correlated with progression in aortic valve calcification (Tables 6.3 and 6.4).

There was a weak negative correlation with smoking $(\mathrm{p}=0.03)$, the presence of vascular disease $(\mathrm{p}=0.01)$ and male $\operatorname{sex}(\mathrm{p}=0.03)$. Progression in aortic jet velocity was not associated with the presence of hyperlipidaemia, ischaemic heart disease, smoking, or any of the biochemical markers of atherosclerosis (Tables 6.3 and 6.4). Furthermore, neither progression in aortic jet velocity $(\mathrm{r}=0.03, \mathrm{p}=0.70)$ nor AVC score $(\mathrm{r}=-0.11, \mathrm{p}=0.23)$ correlated with mean on treatment serum LDL cholesterol concentrations [Cowell et al 2005].

\subsubsection{Inflammation}

At baseline, serum CRP concentrations were the same in both treatment groups $(\mathrm{p}=0.76)$, but fell with $80 \mathrm{mg}$ of atorvastatin $(\mathrm{p}=0.001)$. Baseline CRP concentrations did not correlate with baseline severity of stenosis (aortic jet velocity, $\mathrm{p}=0.95$ ), or extent of valvular calcification ( $\log$ AVC score, $\mathrm{p}=0.82$ ). Furthermore neither baseline nor mean on treatment CRP concentrations correlated with disease progression or clinical outcome (Table 6.3). 


\subsubsection{Arterial stiffness}

Augmentation pressure and pulse pressure, but not augmentation index, correlated with age, height, systolic blood pressure, and mean arterial pressure. No measures of arterial stiffness correlated with baseline severity of aortic valve stenosis or valvular calcification, nor were there any associations between arterial stiffness and disease progression.

\subsubsection{Brain natriuretic peptide}

Serum BNP concentrations at baseline were the same in both treatment groups $(\mathrm{p}=0.37)$ and were not influenced by atorvastatin therapy $(\mathrm{p}=0.33)$. However, serum BNP concentrations at baseline did correlate with baseline severity of stenosis $(\mathrm{r}=0.27, \mathrm{p}=0.002)$ as well as with the extent of valvular calcification on $\mathrm{CT}(\mathrm{r}=0.18$, $\mathrm{p}=0.04)$. The progression in aortic jet velocity correlated with basal and rate of change in serum BNP concentrations $(r=0.26, p=0.003$ and $r=0.26, p=0.002$ respectively).

\subsubsection{Clinical OUTCOME}

During the study follow-up period, 43 patients (28\%) reached a pre-defined clinical end-point (Table 6.5). Six patients died, 2 secondary to malignant disease. The remaining 4 were cardiovascular deaths; one patient with severe asymptomatic aortic stenosis collapsed and died of an acute myocardial infarction (diagnosed at postmortem), one patient with severe symptomatic aortic stenosis who had declined surgery had a sudden cardiac death, and 2 patients died in hospital whilst awaiting urgent valve replacement for severe symptomatic stenosis associated with heart failure. 
TABLE 6.5 Clinical end-points

\begin{tabular}{lcc}
\hline \multicolumn{1}{c}{ Clinical end-points } & $\begin{array}{c}\text { Number } \\
\mathbf{n}=\mathbf{1 5 5}\end{array}$ & $\begin{array}{c}\text { Per cent } \\
\%\end{array}$ \\
\hline Deaths & $\mathbf{6}$ & $\mathbf{3 . 8}$ \\
Cardiovascular & 4 & $\mathbf{1 9 . 4}$ \\
Other & 2 & \\
Aortic valve replacement & $\mathbf{3 0}$ & \\
Severe symptomatic aortic stenosis & 28 & \\
Severe asymptomatic aortic stenosis prior to non-cardiac surgery & 1 & $\mathbf{4 . 5}$ \\
Aortic root dilatation and mild aortic stenosis & 1 & \\
Severe symptomatic aortic stenosis & $\mathbf{7}$ & \\
Referred for AVR & 5 & $\mathbf{2 7 . 7}$ \\
For medical management & $\mathbf{4 3}$ & \\
Total number end-points reached & 2 & \\
\hline
\end{tabular}

AVR - aortic valve replacement. 
Thirty patients underwent AVR, 28 of which were for severe symptomatic aortic stenosis. One patient underwent AVR for severe but asymptomatic aortic stenosis prior to lung resection, and another for mild aortic stenosis and a dilated aortic root.

Seven further patients developed symptoms secondary to severe aortic stenosis, of whom five were awaiting valve surgery at the time of study completion. The remaining 2 patients were deemed unfit for major cardiac surgery due to frailty and co-morbidity.

Overall fewer clinical end-points appeared to have been reached in the statin treated group, however numbers were small and the difference was not statistically significant (Chapter 4) [Cowell et al 2005]. Clinical outcome was only predicted by baseline and subsequent rate of progression of aortic stenosis severity and calcification $(p<0.001)$, as well as by baseline $(p=0.02)$ and rate of progression $(\mathrm{p}<0.001)$ of serum BNP concentrations. However, neither clinical nor biochemical markers of atherosclerosis and cardiovascular risk, including serum cholesterol and CRP concentrations were associated with clinical outcome (Table 6.6 and 6.7). 


\begin{tabular}{|c|c|c|c|}
\hline & No Event & Event & $\begin{array}{c}t \text {-test } \\
\text { P value }\end{array}$ \\
\hline \multicolumn{4}{|l|}{ Baseline demographics } \\
\hline Age (years) & $67(11)$ & $69(9)$ & 0.47 \\
\hline Height $(\mathrm{cm})$ & 168 & 169 & 0.46 \\
\hline Weight (Kg) & $79(15)$ & $81(15)$ & 0.41 \\
\hline \multicolumn{4}{|l|}{ Blood Pressure } \\
\hline Baseline systolic BP & $145(20)$ & $141(18)$ & 0.19 \\
\hline Baseline diastolic BP & $82(11)$ & $81(11)$ & 0.36 \\
\hline Mean study systolic BP & $143(18)$ & $138(16)$ & 0.14 \\
\hline Mean study diastolic BP & $81(8)$ & $80(11)$ & 0.77 \\
\hline \multicolumn{4}{|l|}{ Disease severity } \\
\hline Baseline Vmax & $3.20(0.53)$ & $4.0(0.54)$ & $<0.001$ \\
\hline Baseline CT AVC & $6686(7351)$ & $11401(9081)$ & 0.001 \\
\hline Baseline Log CT AVC & $3.57(0.55)$ & $3.93(0.35)$ & $<0.001$ \\
\hline \multicolumn{4}{|l|}{ Disease progression } \\
\hline Change peak velocity/yr & $0.13(0.16)$ & $0.37(0.21)$ & $<0.001$ \\
\hline Change absolute $\mathrm{AVC} / \mathrm{yr}$ & $1129(1328)$ & $2850(2429)$ & $<0.001$ \\
\hline Change log AVC/yr & $0.09(0.12)$ & $0.10(0.06)$ & 0.68 \\
\hline Per cent change $\mathrm{AVC} / \mathrm{yr}$ & $28.7(32.7)$ & $30.7(23.6)$ & 0.73 \\
\hline \multicolumn{4}{|l|}{ Baseline serum biochemistry } \\
\hline Urea $(\mathrm{mmol} / \mathrm{L})$ & $6.7(5.8)$ & $6.1(1.7)$ & 0.52 \\
\hline Creatinine $(\mu \mathrm{mol} / \mathrm{L})$ & $92(22)$ & $92(19)$ & 0.86 \\
\hline Calcium $(\mathrm{mmol} / \mathrm{L})$ & $2.29(0.11)$ & $2.29(0.10)$ & 0.97 \\
\hline Total cholesterol (mmol/L) & $5.7(0.9)$ & $5.7(1.1)$ & 0.98 \\
\hline HDL cholesterol (mmol/L) & $1.5(0.5)$ & $1.6(0.4)$ & 0.25 \\
\hline LDL cholesterol (mmol/L) & $3.5(0.8)$ & $3.5(1.0)$ & 0.96 \\
\hline \multicolumn{4}{|l|}{$\begin{array}{l}\text { Mean on treatment serum } \\
\text { biochemistry }\end{array}$} \\
\hline Urea $(\mathrm{mmol} / \mathrm{L})$ & $6.2(1.9)$ & $6.2(1.7)$ & 0.96 \\
\hline Creatinine $(\mu \mathrm{mol} / \mathrm{L})$ & $92(21)$ & $93(24)$ & 0.68 \\
\hline Calcium $(\mathrm{mmol} / \mathrm{L})$ & $2.29(0.09)$ & $2.29(0.10)$ & 0.86 \\
\hline Total Cholesterol $(\mathrm{mmol} / \mathrm{L})$ & $4.6(1.3)$ & $4.7(1.5)$ & 0.86 \\
\hline HDL cholesterol (mmol/L) & $1.4(0.4)$ & $1.5(0.4)$ & 0.26 \\
\hline LDL cholesterol (mmol/L) & $2.8(1.2)$ & $2.8(1.3)$ & 0.83 \\
\hline \multicolumn{4}{|l|}{ C-reactive protein } \\
\hline Log baseline CRP & $0.42(0.50)$ & $0.32(0.48)$ & 0.32 \\
\hline Log mean study CRP & $0.28(0.54)$ & $0.41(0.52)$ & 0.23 \\
\hline Change log CRP/year & $-0.08(0.18)$ & $0.03(0.33)$ & 0.016 \\
\hline \multicolumn{4}{|l|}{ Brain natriuretic peptide } \\
\hline Log baseline BNP & $2.30(0.56)$ & $2.54(0.38)$ & 0.02 \\
\hline Rate of change BNP/yr & $0.08(0.13)$ & $0.18(0.20)$ & $<0.001$ \\
\hline
\end{tabular}


TABLE 6.7 Clinical risk factors predicting clinical outcome

\begin{tabular}{lccc}
\hline Clinical Risk Factors & $\begin{array}{c}\text { No Event } \\
(\mathbf{n}=\mathbf{1 1 2})\end{array}$ & $\begin{array}{c}\text { Event } \\
(\mathbf{n}=\mathbf{4 3})\end{array}$ & $\begin{array}{c}\text { Chi'-test } \\
\text { p value }\end{array}$ \\
\hline Hyperlipidaemia & $7(6)$ & $3(7)$ & 0.89 \\
Hypertension & $56(50)$ & $23(53)$ & 0.83 \\
$\begin{array}{l}\text { Ischaemic heart disease } \\
\text { Diabetes mellitus }\end{array}$ & $23(20)$ & $7(16)$ & 0.71 \\
$\begin{array}{l}\text { Peripheral and, or } \\
\text { cerebrovascular disease }\end{array}$ & $3(3)$ & $2(5)$ & 0.91 \\
$\begin{array}{l}\text { Current smoker } \\
\text { Male sex }\end{array}$ & $21(19)$ & $9(21)$ & 0.94 \\
\hline
\end{tabular}

Categorical variables stated as, $\mathbf{n}(\%)$. 


\subsection{DISCUSSION}

Using a cohort of patients with aortic stenosis, we have prospectively demonstrated that the major predictors of disease progression and clinical outcome remain measures of disease severity; namely aortic jet velocity, aortic valve calcification and serum BNP concentration. With the exception of hypertension, the presence of atherosclerotic risk factors and vascular disease were not predictive.

It is clear that patients with symptomatic aortic stenosis require urgent aortic valve surgery. The timing of surgery in patients with asymptomatic severe aortic stenosis remains controversial and clinical practice varies. Given the morbidity and mortality associated with valve surgery, many argue that surgery should be delayed until the onset of symptoms. The concern is that patients do not always accurately report the onset of symptoms. This may arise because patients do not recognise the significance of symptoms, particularly mild dyspnoea or fatigue that may be wrongly attributed to age, or subconsciously reduce activity levels in order to avoid symptoms. It would therefore be useful to have biochemical or clinical markers that help identify those patients with severe disease who might benefit from prompt aortic valve surgery.

\subsubsection{C-REACTIVE PROTEIN}

Serum CRP concentration is a sensitive but non-specific marker of systemic inflammation that is elevated in patients with vascular disease [Van der Meer et al 2002]. It is an independent predictor of future cardiovascular events in healthy adults and in those with established disease [Ridker et al 1998]. Furthermore serum CRP concentrations are elevated in patients with calcific aortic stenosis, even in the 
absence of other overt vascular disease [Galante et al 2001]. In severe aortic stenosis, valvular CRP concentrations correlate with serum CRP concentrations [Skowash et al 2006], and serum CRP concentrations fall following AVR [Gerber et al 2003]. This is in keeping with histological studies suggesting that the aortic valve is a site of active inflammation. Could serum CRP concentrations provide a marker to predict disease progression or clinical outcome in patients with calcific aortic stenosis?

Serum CRP concentrations do not correlate with the severity of aortic stenosis [Gunduz et al 2003] and no study has to date evaluated its relationship with clinical outcome. One preliminary study has suggested that disease progression was more rapid in those with higher CRP concentrations [Sanchez et al 2006 ] but conclusions were limited by the small sample size ( $n=\sim 20$ per group), length of follow-up (6 months) and the single baseline CRP measurement. We have here looked at relationship between serum CRP concentrations and (a) the severity of valvular stenosis at baseline, (b) the rate of disease progression, and (c) clinical outcome. We were unable to demonstrate any association between serum CRP concentrations and any of these measures despite being able to reproduce the well-described reductions of serum CRP concentrations with atorvastatin therapy in our patient group [Houslay et al 2006].

\subsubsection{ARTERIAL STIFFNESS}

Decreased vascular distensibility is a marker of arterial disease and is associated with increased cardiovascular risk. Arterial stiffness increases with age [Mitchell et al 2004] and smoking [Mahmud and Feely 2003], and is associated with certain disease

states including atherosclerosis [van Popele et al 2001], hypertension [Laurent et al 
1994], coronary artery disease [Hayashi et al 2002] diabetes mellitus [Salomaa et al 1995], and hypercholesterolaemia [Wilkinson et al 2002]. Clinical studies demonstrate that increased arterial stiffness is an independent predictor of cardiovascular risk [Weber et al 2005], premature coronary artery disease [Weber $e t$ al 2004], and mortality in patients with hypertension [Laurent et al 2001] and endstage renal failure [Blacher et al 1999; London et al 2001]. Little is known about the associations between aortic stenosis and arterial stiffness but given that calcific aortic stenosis is a condition associated with progressive rigidity and thickening of the aortic valve cusps [Newby et al 2006], we wished to explore whether there was a relationship between arterial stiffness and aortic stenosis and whether this could be used to predict disease progression or clinical outcome.

One small study $(\mathrm{n}=30)$ has previously suggested that invasive measurements of aortic pulse wave velocity correlate with the severity of aortic stenosis (Liu et al 2004). However, we were unable to find any association between noninvasive measures of arterial stiffness, and basal severity, disease progression or clinical outcome. In a limited number of patients $(n=18)$, we did measure pulse wave velocity but again demonstrated no associations with disease severity, progression or outcome. This lack of association suggests that the pathophysiological processes underlying arterial stiffness and aortic stenosis are distinct and independent.

We do accept that we may have missed a weak association and that many of the principles and assumptions employed by pulse wave analysis to generate measures of arterial stiffness may not be valid in patients with aortic stenosis. Nevertheless, we 
were able to confirm previous associations between measures of arterial stiffness and age, height and blood pressure.

\subsubsection{BRAIN NATRIURETIC PEPTIDE}

Brain natriuretic peptide is elevated in both symptomatic and asymptomatic left ventricular dysfunction, and serum concentrations correlate with NYHA class and prognosis [Tsutamoto et al 1997; McDonagh et al 2001; Berger et al 2002; Lubien et al 2002]. Serum BNP concentrations are also elevated in other structural heart disease [Nakamura et al 2002] including left ventricular hypertrophy and aortic stenosis. Recent studies have demonstrated that serum BNP concentrations correlate with the severity of aortic stenosis and NYHA class [Gerber et al 2003; Lim et al 2004]. In patients with severe aortic stenosis, BNP is an independent predictor of symptom free survival [Bergler-Klein et al 2004] and clinical outcome [Lim et al 2004].

We have established, for the first time, that basal and subsequent increases in serum BNP concentrations predict both disease progression and clinical outcome in a large cohort of patients. This predictive power is perhaps not unsurprising given that serum BNP concentrations correlate with the baseline severity of aortic stenosis, which in

turn is a major predictor of disease progression and clinical outcome. It is likely therefore that serum BNP concentrations are acting as a marker of disease severity through the association with left ventricular hypertrophy and systolic dysfunction. 


\subsubsection{STUDY LIMITATIONS}

In the SALTIRE trial, we excluded patients with coronary artery disease who were receiving or had an indication for statin therapy. Although atherosclerotic risk factors may have a more important role in the progression of aortic stenosis in these patients, they will require risk factor management irrespective of their valvular heart disease. Moreover, our patient population was representative of the population of patients with aortic stenosis seen in clinical practice with a broad age range and significant cardiovascular co-morbidity.

We have to date conducted only univariate analyses. Given the potential interdependency of some of the variables, a step-wise multiple logistic regression analysis is underway. This may help identify independent predictors of disease progression and clinical outcome, but is unlikely to alter our main conclusions.

\subsection{CONCLUSIONS}

The major predictors of disease progression and clinical outcome in patients with aortic stenosis remain measures of disease severity. Estimation of serum BNP concentration may be helpful in the monitoring of patients with severe aortic stenosis

particularly where there is clinical uncertainty or where incomplete clinical information is available such as poor quality echocardiographic imaging. 


\section{CHAPTER 7}

\section{CONCLUSIONS AND FUTURE DIRECTIONS}




\subsection{CONCLUSIONS}

\subsubsection{Helical COMPUTED TOMOGRAPHY IN THE QUANTIFICATION OF AORTIC}

VALVE CALCIUM

Severity of valvular calcification has been reported to be a strong predictor of clinical outcome in patients with aortic stenosis. Echocardiography is the gold standard for assessing patients with valvular heart disease, but only provides a semiquantitative measure of calcification. Computed tomography has recently been identified as a potential tool for more accurate quantification of AVC content. Electron beam CT derived calcium scores demonstrate good reproducibility with interscan variation of 7-9\% [Pohle et al 2001; Budoff et al 2002], and correlate closely with calcium deposition in excised valvular tissue [Messika-Zeitoun et al 2004].

At inception of the SALTIRE trial, reproducibility of AVC scores quantified by helical CT had not been determined, nor had there been a direct comparison with electron beam CT. A number of studies quantifying coronary artery calcium content have subsequently demonstrated a good correlation between calcium scores determined using multi-slice and electron beam CT [Carr et al 2000; Becker et al 2001].

We have demonstrated that the measurement of AVC content using helical CT reveals very good interscan reproducibility when assessed in a cohort of 20 patients undergoing repeated assessment. Our figures indicate that helical CT is a useful tool 
for determining the severity of valvular calcification and in monitoring the progression of calcium accumulation. This suggests that it may be valuable in assessing the influence of pharmacological therapy on disease progression.

\subsubsection{RELATIONSHIP BETWEEN AORTIC VALVE CALCIUM CONTENT AND SEVERITY OF AORTIC STENOSIS}

In patients with aortic stenosis, disease progression and clinical outcome are influenced by baseline severity of stenosis, as well as the extent of valvular calcification [Davies and Gershlick 1991; Bahler et al 1999; Rosenhek et al 2000]. Until recently the relationship between the Doppler-derived severity of stenosis and extent of valve calcification was not clear. Calcification of the aortic valve reduces leaflet flexibility, with a resultant acceleration of blood flow across the narrowed orifice, and hence an increase in aortic jet velocity. It is important to remember that jet velocity will be determined by other factors influencing the haemodynamic load on the left ventricle including the presence of hypertension or coronary artery disease. Although an association between valve calcification and severity of stenosis seems likely, this had not been clearly demonstrated in a prospective trial.

We have prospectively demonstrated in a large cohort of patients that severity of stenosis determined by Doppler echocardiography is strongly correlated with the degree of aortic valve calcification on helical CT. This suggests that the CT derived calcium score provides complementary information to the jet velocity, and that AVC accumulation over time may provide additional prognostic information independent of the aortic jet velocity. 


\subsubsection{INFLUENCE OF STATIN THERAPY ON THE PROGRESSION OF CALCIFIC AORTIC}

STENOSIS

Hydroxymethylglutaryl coenzyme A reductase inhibitors or statins are now well established in the primary and secondary prevention of coronary artery disease. Several studies have suggested that these drugs can cause regression of coronary artery disease as well as reduce the calcific volume of coronary plaques [Callister $e t$ al 1998]. Given the clinical association of calcific aortic stenosis with hyperlipidaemia and coronary artery disease, and the striking histological similarities with atheroma, the speculation that statins may have the potential to influence disease progression in aortic stenosis is an intriguing hypothesis [Mohler 2000].

Recent retrospective studies [Aronow et a 2001; Novaro et al 2001; Bellamy et al 2002; Rosenhek et al 2004] have suggested that statins may delay disease progression in aortic stenosis (Table 7.1) through their lipid-lowering and antiinflammatory actions. These observational data should be interpreted with caution since none of these studies were prospective randomised trials, serum LDL cholesterol concentrations did not correlate with disease progression, and the statin doses were small. There may also be some publication bias with studies reporting negative findings under-represented in the literature [Samal et al 2002; AntoniniCanterin et al 2005]. 


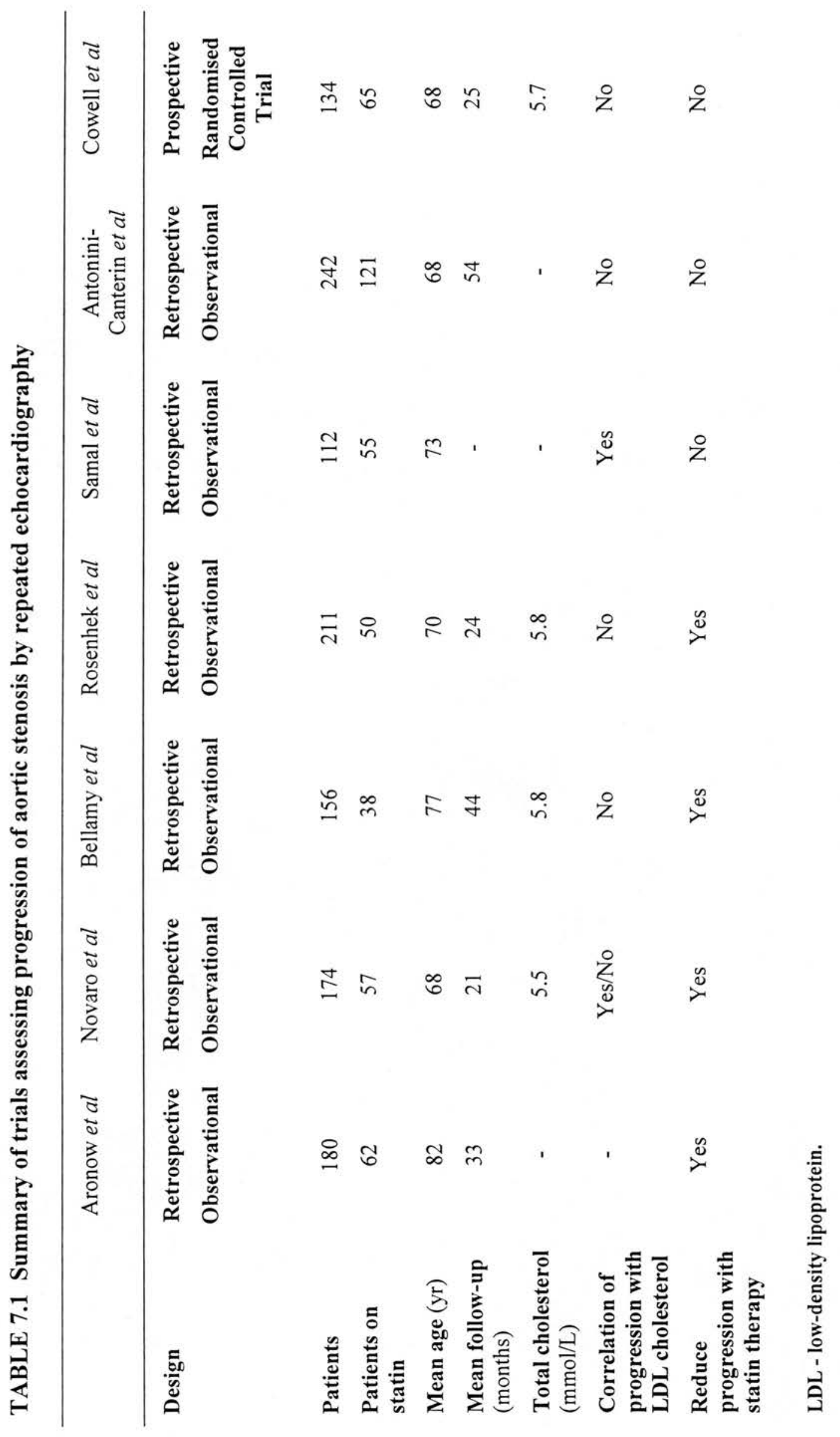


The SALTIRE trial [Cowell et al 2005] is the first double-blind randomised controlled trial of lipid-lowering therapy to be completed in patients with calcific aortic stenosis. This trial of 155 patients demonstrated that, whilst atorvastatin $80 \mathrm{mg}$ daily more than halved serum LDL cholesterol concentrations, it did not halt the progression or induce regression of the valvular disease process as measured by both Doppler echocardiography and helical CT. Indeed no relationship between serum LDL cholesterol concentrations and the progression of aortic stenosis was apparent, nor was there a demonstrable effect of high dose atorvastatin on clinical end-points. Thus, irrespective of the method of assessing disease progression, we have consistently demonstrated the continued deterioration of aortic stenosis despite intensive reductions in serum cholesterol concentrations.

\subsubsection{INFLUENCE OF STATIN THERAPY ON THE PROGRESSION OF CORONARY}

\section{ARTERY CALCIFICATION}

Coronary artery calcification is an independent risk factor for coronary heart disease, and progresses over time at a rate of 20-30\% per year [Callister et al 1998; Budoff et al 2000; Schmermund et al 2001]. Calcium burden may be determined using CT, and quantity of coronary artery calcium correlates with overall coronary plaque burden [Mautner et al 1994; Rumberger et al 1995; Sangiorgi et al 1998]. Several nonrandomised observational studies have reported a reduction in coronary calcium accumulation in patients taking statin therapy [Callister et al 1998; Achenbach et al 2002], but no placebo-controlled trials have been published substantiating these results. 
In using helical CT to determine AVC content in the SALTIRE trial, we had the opportunity to assess prospectively the effect of intensive lipid-lowering therapy on coronary calcification. We demonstrated good reproducibility of coronary scores and progression of coronary artery calcification by $20 \%$ per year. However, intensive lipid-lowering therapy did not delay the progression or induce regression of coronary artery calcium burden. This is despite a $50 \%$ reduction in serum LDL cholesterol and CRP concentrations.

Our findings indicate that earlier observational studies have overestimated the potential beneficial effects of statins on coronary artery calcification, and indeed more recent prospective randomised controlled trials [Arad et al 2005; Raggi et al 2005] have also failed to find an effect. Although coronary artery calcium scores correlate well with the presence of atherosclerosis and predict future coronary risk, our findings indicate that there is currently no role for monitoring progression of coronary artery calcification to assess the effects of lipid-lowering therapy out with a clinical trial setting.

\subsubsection{FACTORS INFLUENCING THE PROGRESSION OF AORTIC STENOSIS AND}

\section{CLINICAL OUTCOME}

Calcific aortic stenosis is common, and gradually progresses over time. Rates of disease progression vary widely between individuals, and as soon as patients with severe stenosis develop symptoms, AVR is indicated. In general, patients with milder forms of disease have a better outlook, but there is an increased risk of unrelated cardiovascular events. 
Mechanical injury is thought to initiate the disease process, inducing endothelial disruption that is followed by an inflammatory process closely resembling atherosclerosis. In keeping with this observation, the clinical risk factors for aortic stenosis include those for cardiovascular disease, as well as conditions affecting calcium metabolism. However, the clinical factors associated with disease progression are less well defined, with many mainly retrospective studies reporting conflicting outcomes. Clinical outcome, however, is strongly predicted by baseline aortic jet velocity, rate of change in jet velocity, the extent of valvular calcification and functional status [Otto et al 1997; Rosenhek et al 2000]. We wished to define predictors of disease progression and clinical outcome more clearly, and to identify new modifiable markers.

In patients with calcific aortic stenosis we have prospectively demonstrated that the major predictors of disease progression and clinical outcome remain measures of disease severity; namely aortic jet velocity, aortic valve calcification and serum BNP concentration. With the exception of hypertension, the presence of atherosclerotic risk factors and vascular disease were not predictive.

We have established, for the first time, that basal and subsequent increases in serum BNP concentrations predict both disease progression and clinical outcome in a large cohort of patients. This predictive power is perhaps not unsurprising given that serum BNP concentrations correlate with the baseline severity of aortic stenosis, which in turn is a major predictor of disease progression and clinical outcome. It is likely therefore that serum BNP concentrations are acting as a marker of disease 
severity through the association with left ventricular hypertrophy and systolic dysfunction.

\subsection{FUTURE DIRECTIONS AND CLINICAL IMPLICATIONS}

\subsubsection{COMPUTED TOMOGRAPHY IN THE ASSESSMENT OF AORTIC VALVE}

\section{CALCIFICATION AND STENOSIS}

Although we have clearly demonstrated that helical (two detector array, or doublehelix) $\mathrm{CT}$ is a reproducible means of measuring valvular calcium content, it is unlikely that such scanners will be utilised in the future given rapid technological advances that have taken place in recent years. Factors influencing the reproducibility of calcium scores include cardiac and respiratory motion artefact, method of image acquisition and reconstruction, the use of phantom calibration, and the method of calcium score calculation. The latest multi-slice scanners with up to 64 detector arrays acquire multiple images at different levels simultaneously in subsecond time, and reconstruct images using ECG gating. Movement artefact and partial-volume effects are reduced [Willmann et al 2002; Morgan-Hughes et al 2003], and it scems logical to assume that more accurate quantification of valvular calcification and improved specificity will result.

Alternative methods of quantifying calcification have been evaluated, including volume and mass scores that have been shown to improve the reproducibility of coronary calcium scores [Callister et al 1998]. These scoring methods have also been utilised in recent aortic valve studies [Morgan-Hughes et al 2003; Koos et al 2005], 
but further evaluation is required to determine which is the most reproducible using the very latest scanning equipment.

Computed tomography imaging has improved to the extent that direct measurement of aortic stenosis severity is now also possible. Valve area determined by planimetry, is comparable to that derived by transthoracic and transoesophageal echocardiography [Alkadhi et al 2006; Feuchtner et al 2006]. Given the radiation doses involved, it is unlikely that CT will supersede echocardiography in the assessment of stenosis severity, it may however, prove a useful non-invasive technique in patients with poor ultrasound images.

\subsubsection{POTENTIAL THERAPIES OR AORTIC VALVE STENOSIS}

The current management of patients with aortic stenosis includes monitoring disease progression, and ensuring patient awareness of the need for antibiotic prophylaxis against the relatively low risk of infective endocarditis. For those patients with severe symptomatic disease, AVR is a priority with conventional medical therapy reserved for symptom control in inoperable cases. However, the majority of patients with aortic stenosis do not have symptoms or an indication for surgery. Are there any interventions that can halt or slow the progression of the disease process? Theoretically, anti-inflammatory and anti-proliferative agents would be anticipated to alter the natural history of aortic stenosis. Statin and ACE inhibitor therapies are two commonly used treatments that have proven secondary preventative benefits in cardiovascular disease and exhibit some of these desirable anti-inflammatory and anti-proliferative properties. 


\subsubsection{Hydroxymethylglutaryl coenzyme A reductase inhibitors (statins)}

In the SALTIRE trial, intensive lipid-lowering therapy with $80 \mathrm{mg}$ atorvastatin daily did not halt the progression or induce regression of calcific aortic stenosis. Given the data linking aortic stenosis with atherosclerosis and hypercholesterolaemia, this result is surprising. One potential explanation is that, whilst these features may drive the initiation of aortic stenosis, disease progression may be dependent upon other factors. The aortic valve is subject to continuous dynamic mechanical stress, and leaflet plasticity and structure can have an overriding influence, such as with a bicuspid valve. Moreover, in contrast to atherosclerosis (Figure 7.1), aortic stenosis is associated with a virtual absence of smooth muscle cell proliferation and lipidladen macrophages, and dominated by earlier and more extensive mineralisation. Decreasing the lipid pool and increasing the fibrous cap may be less relevant to the progression of aortic stenosis than it is for the reduction in atherosclerotic plaque rupture with statin therapy in patients with coronary heart disease. Whilst stabilisation and calcification of plaques is beneficial and reduces cardiovascular events in patients with coronary artery disease, reduced inflammation, promotion of healing and increased calcification may lead to progression of aortic stenosis.

It could be argued that lipid-lowering therapy is unlikely to influence disease progression in the presence of significant aortic stenosis. Patients with aortic velocities below $2.5 \mathrm{~m} / \mathrm{s}$ were excluded from the SALTIRE trial and intervening at 


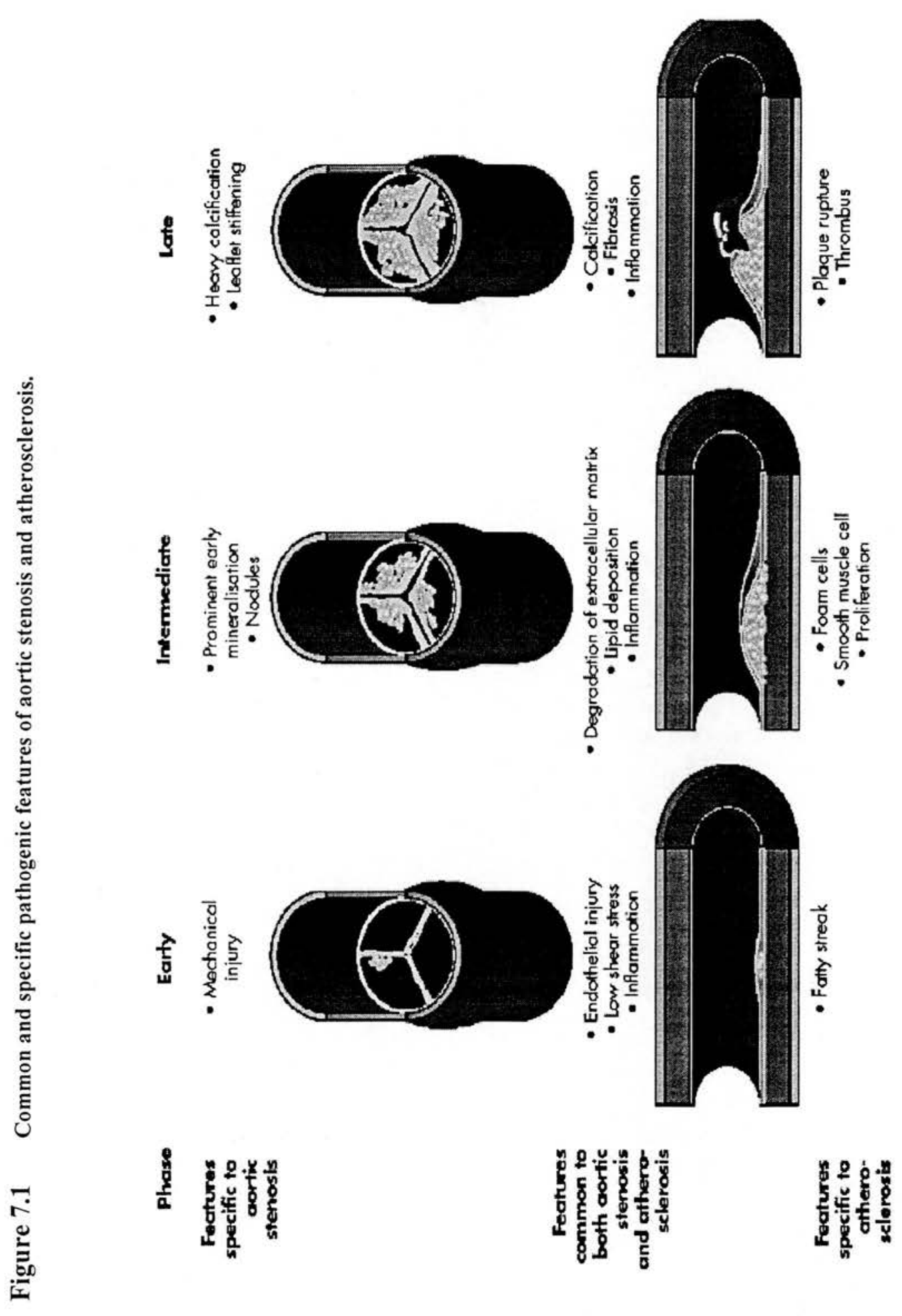


this earlier stage of the disease process may have been more beneficial. However, such patients do not commonly present to routine clinical practice and their identification would potentially require population screening. Moreover, the SALTIRE trial was unable to exclude a modest treatment benefit (a delay in disease progression of $<0.07 \mathrm{~m} / \mathrm{s} / \mathrm{yr}$ or $<5 \%$ aortic valve calcification/yr). Although such modest reductions are unlikely to be meaningful in the majority of older patients, a small decrease in disease progression may be clinically important in younger patients with mild disease who may progress over many years. Indeed, a small preliminary observational study suggests that statin therapy may reduce disease progression in patients with aortic sclerosis [Antonini-Canterin et al 2005] and this finding warrants prospective evaluation.

Statin therapy in patients with aortic stenosis may confer secondary preventative benefits that are independent of its effects on the valvular disease process because of the association between aortic stenosis and coronary artery disease. The SALTIRE trial was not powered to assess the benefits of lipid-lowering therapy on cardiovascular end-points, such as non-fatal and fatal myocardial infarction, but there was a trend in favour of reduced clinical events. It is likely that aortic stenosis and sclerosis may be important markers of occult vascular disease and thereby identify patients who would gain from the preventative benefits of statin therapy. Several larger clinical end-point trials (including the SEAS and ASTRONOMER trials [Rajamannan 2006]) are currently underway that will be able to address this issue. 
Finally, for many patients with aortic stenosis, the first symptom to develop is chest pain and this precipitates the decision to undertake AVR. However, this may be driven by concomitant coronary artery disease rather than progression of valvular stenosis. Previous secondary prevention trials in coronary heart disease have reported a reduction in coronary artery bypass graft surgery rates [The Heart Protection Study Collaborative Group 2002]. Thus, the larger clinical end-point trials of statin therapy in aortic stenosis may suggest a reduction in the rate of valve surgery but this may be driven by patients with aortic stenosis who undergo combined aortic valve and bypass surgery for symptoms of angina pectoris. If statin therapy truly reduces disease progression then a reduction in isolated AVR would be anticipated.

At present we cannot recommend the use of statin therapy in patients with isolated aortic stenosis in the absence of other cardiovascular risk factors. Indeed recently published American Heart Association and American College of Cardiology guidelines suggest awaiting the outcome of further prospective trials in patients with

mild disease that are followed up for a longer period [Bonow et al 2006]. Furthermore, evaluation of the effects of statins in younger patients with aortic valve sclerosis, prior to the development of significant haemodynamic change and progression to valve stenosis, will be required.

\subsubsection{Angiotensin-converting enzyme inhibition}

There are several reasons to believe that ACE inhibitor therapy may have a role in the management of patients with aortic stenosis. First, in contrast to normal valves, sclerotic aortic valves have demonstrable tissue expression of angiotensin II and 
ACE [Helske et al 2004; O'Brien et al 2005] and these may contribute to valvular inflammation, calcification and disease progression. Second, the pressure overload induced by aortic stenosis has several effects on the myocardium including left ventricular hypertrophy, apoptosis and fibrosis. This may accelerate the left ventricular systolic and diastolic dysfunction associated with aortic stenosis. Animal models of outflow obstruction using aortic banding have demonstrated improvement in diastolic function [Litwin et al 1995] and survival [Weinberg et al 1994; Turcani and Rupp 2000] with ACE inhibitor treatment, and in patients with aortic stenosis intra-coronary infusion of ACE inhibitor resulted in immediate improvement in diastolic function [Friedrich et al 1994]. This suggests that ACE inhibitors have the potential to influence clinical outcome through their effect on left ventricular diastolic function and remodelling. Finally, blood pressure lowering will indirectly reduce the pressure overload of the left ventricle as well as potentially reduce the mechanical stress and strain on the aortic valve.

Two preliminary observational studies with ACE inhibitor therapy in patients with aortic stenosis have been conflicting. In a retrospective analysis of 211 patients, Rosenhek and colleagues [Rosenhek et al 2004] failed to demonstrate a delay in the progression of aortic stenosis in patients maintained on ACE inhibitor therapy. Furthermore, the presence of hypertension did not appear to influence the outcome. In contrast, O'Brien and colleagues [O'Brien et al 2005] found that ACE inhibitor therapy was associated with a $71 \%$ reduction in the progression of aortic valve calcification in 123 patients with aortic stenosis undergoing electron beam CT. 
However, such retrospective observational data are difficult to interpret and the study findings have wide confidence intervals.

Historically, ACE inhibition was said to be contraindicated in patients with aortic stenosis. This has primarily been due to the concern of invoking profound peripheral vasodilatation that would result in haemodynamic compromise, collapse and potentially death. However, ACE inhibitors are very well tolerated when initiated in patients with aortic stenosis [Chockalingham et al 2004; O'Brien et al 2004] and many patients $(\sim 30 \%)$ with aortic stenosis are unknowingly established on such therapy without compromise. Indeed, the use of ACE inhibitors appears to confer long-term survival benefit in patients considered to have a contraindication including those with aortic stenosis [Ahmed et al 2005]. The potential beneficial haemodynamic and cardiac effects of ACE inhibition are increasingly being recognised [Routledge and Townsend 2001] and warrant further study in patients with aortic stenosis.

\subsubsection{STATIN THERAPY AND CORONARY ARTERY CALCIFICATION}

Despite the evidence that statin therapy is extremely successful in the primary and secondary prevention of cardiovascular disease, we have not demonstrated a beneficial effect of statin therapy on coronary artery calcification. It has been suggested that calcified atherosclerotic plaques may be relatively more stable [Mintz et al 1995], indicating a possible protective role of calcification in coronary plaques. Statin therapy is thought to produce many of its beneficial effects through plaque stabilisation. Thus vascular calcification may play a role in the initial stabilisation of 
atherosclerotic plaques. This is consistent with our findings and would account for the lack of effect on the progression of coronary artery calcification despite a reduction in serum CRP concentrations.

After the initial stabilisation of the atherosclerotic plaque, it would be anticipated that subsequent progression of coronary calcification would be inhibited. The present study was brief, and follow-up was only continued for a median of 2 years. It would be important to extend our observations to 5 or more years to assess properly the impact of statin therapy on the long-term progression of coronary artery calcification. However, it should be acknowledged that the clinical benefits of statin therapy are apparent within the first few years [Lewis et al 1998; The LIPID Study Group 1998; The Heart Protection Study Collaborative Group 2002] and in some cases the first few months [Schwartz et al 1998] of therapy. Moreover, the St Francis Heart Study demonstrated no beneficial effects despite 4.3 years of follow-up [Arad et al 2005].

\subsubsection{Disease progression aNd Clinical OUTCOME IN CALCIFIC AORTIC} STENOSIS

Aortic stenosis is the commonest adult heart valve condition seen in the western world. It is a gradually progressive disease, characterised by a long asymptomatic phase, followed by a shorter symptomatic phase usually associated with severe narrowing of the aortic valve orifice. Despite the favourable outlook in those patients with mild asymptomatic disease, there is an increased risk of cardiovascular events unrelated to the aortic valve disease. 
Historically, calcific aortic stenosis has been attributed to prolonged "wear and tear" and age-associated valvular degeneration. However, recent evidence suggests that calcific aortic stenosis may result from an active inflammatory process resembling atherosclerosis (Figure 7.1) but also involving biochemical, humoral and genetic factors. Endothelial injury or disruption is thought to allow lipids to penetrate the valvular interstitial tissue [O'Brien et al 1996] and accumulate in areas of inflammation [O'Brien et al 1996; Olsson et al 1999]. Mineralisation is a characteristic of both atherosclerotic and aortic valve lesions, and arises in close proximity to areas of inflammation. Histological studies have highlighted the common features but also confirmed differences in the cellular and mineral components of the two lesions.

These differences may, in part, explain why only $40 \%$ of patients with severe aortic stenosis have significant coronary artery disease [Peltier et al Cardiol 2003] and why the majority of patients with coronary artery disease do not have aortic stenosis. As the underlying pathology for the two conditions appears to be similar, it is likely that other unknown factors, including genetic factors, influence the development of valvular as opposed to vascular lesions [Otto and O'Brien 2001].

Predictors of disease progression have not previously been clearly defined. We have now prospectively demonstrated that the strongest predictors of both disease progression and clinical outcome are baseline severity of stenosis, BNP and extent of valve calcification. The more severe the stenosis and the more heavily calcified the valve, the higher the BNP and the faster the rate of disease progression. However, 
although severity of stenosis correlates with the extent of valvular calcification, the spectrum of calcification for a given aortic jet velocity varies widely, and this diversity is likely to be important in evaluating patients approaching the need for AVR.

\subsubsection{FUTURE EVALUATION OF PROGRESSION AND CLINICAL OUTCOME}

Our findings highlight the importance of evaluating stenosis severity, calcium burden and BNP in monitoring patients with aortic stenosis. Larger clinical trials are needed to evaluate their influence on clinical outcome. Identification of patients at highest risk will facilitate appropriate disease monitoring, with the potential to determine optimal timing of AVR and influence surgical outcome.

\subsection{SUMMARY}

Calcific aortic stenosis is no longer regarded as an age-related degeneration, but an active disease process. The need for an alternative to aortic valve replacement is highlighted by the rising prevalence of aortic stenosis. New therapeutic strategies to limit disease progression are needed in order to delay, and potentially avoid, the need for valve surgery. Statin and ACE inhibitor therapies are two potential and promising therapies that may have beneficial effects in patients with aortic stenosis. They may reduce cardiovascular events rather than disease progression per se, but may prove to be valuable preventative therapy. We must, however, await the results of ongoing large randomised controlled trials to define the role of statin therapy, and consider prospective trials of drugs influencing the renin-angiotensin system, and other antiinflammatory agents including aspirin. 


\section{REFERENCES}




\section{REFERENCES}

Abdul-Hamid AR, Mulley GP. Why do so few older people with aortic stenosis have valve replacement surgery? Age Ageing 1999;28:261-4.

Achenbach S, Giesler T, Ropers D, et al. Detection of coronary artery stenoses by contrast-enhanced, retrospectively electrocardiographically-gated, multislice spiral computed tomography. Circulation 2001;103(21):2535-8.

Achenbach S, Ropers D, Pohle K, et al. Influence of lipid-lowering therapy on the progression of coronary artery calcification: a prospective evaluation. Circulation 2002;106(9):1077-82.

Agatston AS, Janowitz WR, Hildner FJ, Zusmer NR, Viamonte M, Jr., Detrano R. Quantification of coronary artery calcium using ultrafast computed tomography. $J$ Am Coll Cardiol 1990;15(4):827-32.

Ahmed A, Centor RM, Weaver MT, Perry GJ. A propensity score analysis of the impact of angiotensin-converting enzyme inhibitors on long-term survival of older adults with heart failure and perceived contraindications. Am Heart $J$ 2005;149(4):737-43.

Alkadhi H, Wildermuth S, Plass A, et al. Aortic stenosis: comparative evaluation of 16-detector row CT and echocardiography. Radiology 2006;240(1):47-55.

Amato MCM, Moffa PJ, Werner KE, Ramires JAF. Treatment decision in asymptomatic aortic valve stenosis: role of exercise testing. Heart 2001;86:381-6.

Antonini-Canterin F, Popescu BA, Huang G, et al. Progression of aortic valve sclerosis and aortic valve stenosis: what is the role of statin treatment? Ital Heart $J$ 2005;6:119-24.

Arad Y, Spadaro LA, Roth M, Newstein D, Guerci AD. Treatment of asymptomatic adults with elevated coronary calcium scores with atorvastatin, vitamin $\mathrm{C}$, and vitamin E: the St. Francis Heart Study randomized clinical trial. J Am Coll Cardiol 2005;46(1):166-72.

Aronow WS, Ahn C, Kronzon I, Goldman ME. Association of coronary risk factors and the use of statins with progression of mild valvular aortic stenosis in older persons. Am J Cardiol 2001;88:693-5.

Aronow WS, Ahn C, Kronzon I, Nanna M. Prognosis of congestive heart failure in patients aged $>62$ years with unoperated severe valvular aortic stenosis. Am $J$ Cardiol 1993;72:846-8. 
Aronow WS, Schwartz KS, Koenigsberg M. Correlation of serum lipids, calcium and phosphorus, diabetes mellitus and history of systemic hypertension with presence or absence of calcified or thickened aortic cusps or root in elderly patients. Am J Cardiol 1987;59:998-9.

Bahler RC, Desser DR, Finkelhor RS, Brener SJ, Youssefi M. Factors leading to progression of valvular aortic stenosis. Am J Cardiol 1999;84(9):1044-8.

Becker CR, Kleffel T, Crispin A, et al. Coronary artery calcium measurement: agreement of multirow detector and electron beam CT. Am J Roentgenol 2001;176(5):1295-8.

Bellamy MF, Pellikka PA, Klarich KW, TajikAJ, Enriquez-Sarano M. Association of cholesterol levels, Hydroxymethylglutaryl coenzyme-A reductase inhibitor treatment, a progression of aortic stenosis in the community. $\mathrm{J} \mathrm{Am} \mathrm{Coll} \mathrm{Cardiol}$ 2002; 40:1723-30.

Beppu S, Suzuki S, Matsuda H, Ohmori F, Nagata S, Miyatake K. Rapidity of progression of aortic stenosis in patients with congenital bicuspid aortic valves. Am J Cardiol 1993;71:322-7.

Berger R, Huelsman M, Strecker K, et al. B-type natriuretic peptide predicts sudden death in patients with chronic heart failure. Circulation 2002;105(20):2392-7.

Bergler-Klein J, Klaar U, Heger M, et al. Natriuretic peptides predict symptom-free survival and postoperative outcome in severe aortic stenosis. Circulation 2004;109(19):2302-8.

Berliner JA, Territo MC, Sevanian A, et al. Minimally oxidised low density lipoprotein stimulates monocyte endothelial interactions. J Clin Invest 1990;85:12606.

Blacher J, Guerin AP, Pannier B, Marchais SJ, Safar ME, London GM. Impact of aortic stiffness on survival in end-stage renal disease. Circulation 1999;99(18):24349.

Bland JM, Altman DG. Statistical methods for assessing agreement between two methods of clinical measurement. Lancet 1986;1:307-10.

Bonow RO, Carabello B, de Leon AC, et al. ACC/AHA guidelines for the management of patients with valvular heart disease. $J$ Am Coll Cardiol 1998;32:1486-1588.

Bonow RO, Carabello BA, Kanu C, et al. ACC/AHA 2006 guidelines for the management of patients with valvular heart disease. Circulation 2006;114(5):e84231.

Boomsma F, van den Meiracker AH. Plasma A- and B-type natriuretic peptides: physiology, methodology and clinical use. Cardiovasc Res 2001;51(3):442-9. 
Boström K, Watson KE, Stanford WP, Demer LL. Atherosclerotic calcification: Relation to developmental osteogenesis. Am J Cardiol 1995;75:88B-91B.

Boudjemline Y, Bonhoeffer P. Steps toward percutaneous aortic valve replacement. Circulation 2002;105:775-8.

Bouma BJ, van den Brink RBA, van der Meulen JHP, et al. To operate or not on elderly patients with aortic stenosis: the decision and its consequences. Heart 1999;82:143-8.

Breen JF, Sheedy PF, Schwartz RS, et al. Coronary artery calcification detected with ultrafast CT as an indication of coronary artery disease. Radiology 1992;185:435-9.

Brener SJ, Duffy CI, Thomas JD, Stewart WJ. Progression of aortic stenosis in 394 patients: Relation to changes in myocardial and mitral valve dysfunction. J Am Coll Cardiol 1995;25:305-10.

Brown H, Prescott RJ. Applied mixed models in medicine. Chichester, England: John Wiley, 1999:239-41.

Budoff MJ, Lane KL, Bakhsheshi $\mathrm{H}$, et al. Rates of progression of coronary calcium by electron beam tomography. Am J Cardiol 2000;86(1):8-11.

Budoff MJ, Mao S, Takasu J, Shavelle DM, Zhao XQ, O'Brien KD. Reproducibility of electron-beam CT measures of aortic valve calcification. Acad Radiol 2002;9(10):1122-7.

Callister TQ, Cooil B, Raya SP, Lippolis NJ, Russo DJ, Raggi P. Coronary artery disease: improved reproducibility of calcium scoring with an electron-beam CT volumetric method. Radiology 1998a;208(3):807-14.

Callister TQ, Raggi P, Cooil B, Lippolis NJ, Russo DJ. Effect of HMG-CoA reductase inhibitors on coronary artery disease as assessed by electron-beam, computed tomography. N Engl J Med 1998b;339:1972-8.

Carabello BA. Evaluation and management of patients with aortic stenosis. Circulation 2002;105:1746-50.

Carr JJ, Crouse JR, 3rd, Goff DC, Jr., D'Agostino RB, Jr., Peterson NP, Burke GL. Evaluation of subsecond gated helical CT for quantification of coronary artery calcium and comparison with electron beam CT. Am J Roentgenol 2000;174(4):91521.

Chambers J. Exercise testing to guide surgery in aortic stenosis. Heart 1999;82:7-8.

Chenillot O, Henny J, Steinmetz J, Herbeth B, Wagner C, Siest G. High sensitivity C-reactive protein: biological variations and reference limits. Clin Chem Lab Med 2000;38(10):1003-11.

Chisholm G. Cytotoxicity of oxidised lipoproteins. Curr Opin Lipidol 1991;2:311-6. 
Chockalingam A, Venkatesan S, Subramaniam T, et al. Safety and efficacy of angiotensin-converting enzyme inhibitors in symptomatic severe aortic stenosis: Symptomatic Cardiac Obstruction-Pilot Study of Enalapril in Aortic Stenosis (SCOPE-AS). Am Heart J 2004;147(4):E19.

Chui MC, Newby DE, Panarelli M, Bloomfield P, Boon NA. Calcific aortic stenosis and hypercholesterolaemia: a causal association? Heart 1999;81 (Abstract):171.

Chui MCK, Newby DE, Panarelli M, Bloomfield P, Boon NA. Association between calcific aortic stenosis and hypercholesterolemia: is there a need for a randomised controlled trial of cholesterol lowering therapy? Clin Cardiol 2001;24:52-5.

Cosmi JE, Kort S, Tunick PA, et al. The risk of the development of aortic stenosis in patients with "benign" aortic valve thickening. Arch Intern Med 2002;162:2345-7.

Cowell SJ, Newby DE, Burton J, et al. Aortic valve calcification on computed tomography predicts the severity of aortic stenosis. Clin Radiol 2003;58:712-6.

Cowell SJ, Newby DE, Elder AT. Calcific aortic stenosis: same old story? Age Ageing 2004;33(6):538-44.

Cowell SJ, Newby DE, Prescott RJ, et al. Scottish Aortic Stenosis and Lipid Lowering Trial, Impact on Regression (SALTIRE) Investigators: A randomized trial of intensive lipid-lowering therapy in calcific aortic stenosis. $N$ Engl $\mathrm{J}$ Med 2005;352(23):2389-97.

Cribier A, Eltchaninoff $\mathrm{H}$, Bash A, et al. Percutaneous transcatheter implantation of an aortic valve prosthesis for calcific aortic stenosis. First human case description. Circulation 2002;106:3006-8.

Cujec B, Pollick C. Isolated thickening of one aortic cusp: preferential thickening of the noncoronary cusp. J Am Soc Echocardiogr 1988;1:430-2.

Daoud AS, Jarmolych J, Augustyn JM, Fritz KE. Sequential morphologic studies of regression of advanced atherosclerosis. Arch Pathol Lab Med 1981;105(5):233-9.

Davies MJ. The Composition of Coronary-Artery Plaques. $N$ Engl $J$ Med 1997;336(18):1312-4.

Davies SW, Gershlick AH, Balcon R. Progression of valvar aortic stenosis: a longterm retrospective study. Eur Heart J 1991;12:10-14.

Demer LL. A skeleton in the atherosclerosis closet. Circulation 1995;92:2029-32.

Demer LL. Cholesterol in vascular and valvular calcification. Circulation 2001;104:1881-3.

Didier D, Ratib O, Lerch R, Friedli B. Detection and quantification of valvular heart disease with dynamic cardiac MR imaging. Radiographics 2000;20(5):1279-99. 
Downs JR, Clearfield M, Weis S, et al. Primary prevention of acute coronary events with lovastatin in men and women with average cholesterol levels: results of AFCAPS/TexCAPS. Air Force/Texas Coronary Atherosclerosis Prevention Study. Jama 1998;279(20):1615-22.

Edep ME, Shirani J, Wolf P, Brown DL. Matrix metalloproteinase expression nonrheumatic aortic stenosis. Cardiovasc Pathol 2000;9:281-6.

Faggiano P, Ghizzoni G, Sorgato A, et al. Rate of progression of valvular aortic stenosis in adults. Am J Cardiol 1992;70:229-33.

Faggiano P, Antonini-Canterin F, Erlicher A, et al. Progression of aortic valve sclerosis to aortic stenosis. Am J Cardiol 2003;91:99-101.

Feuchtner GM, Dichtl W, Friedrich GJ, et al. Multislice computed tomography for detection of patients with aortic valve stenosis and quantification of severity. $\mathrm{J} \mathrm{Am}$ Coll Cardiol 2006;47(7):1410-7.

Friedrich SP, Lorell $\mathrm{BH}$, Rousseau MF, et al. Intracardiac angiotensin-converting enzyme inhibition improves diastolic function in patients with left ventricular hypertrophy due to aortic stenosis. Circulation 1994;90(6):2761-71.

Galante A, Pietroiusti A, Vellini M, et al. C-reactive protein is increased in patients with degenerative aortic valvular stenosis. J Am Coll Cardiol 2001;38(4):1078-82.

Galloway AC, Colvin SB, Grossi EA, et al. Ten-year experience with aortic valve replacement in 482 patients 70 years of age or older: operative risk and long-term results. Ann Thorac Surg 1990;49:84-93.

Gerber IL, Stewart RA, Hammett CJ, et al. Effect of aortic valve replacement on Creactive protein in nonrheumatic aortic stenosis. Am J Cardiol 2003a;92(9):1129-32.

Gerber IL, Stewart RA, Legget ME, et al. Increased plasma natriuretic peptide levels reflect symptom onset in aortic stenosis. Circulation 2003b;107(14):1884-90.

Ghaisas NK, Foley JB, O'Briain DS, Crean P, Kelleher D, Walsh M. Adhesion molecules in nonrheumatic aortic valve disease: endothelial expression, serum level and effects of valve replacement. J Am Coll Cardiol 2000;36:2257-62.

Gilbert T, Orr W, Banning AP. Surgery for aortic stenosis in severely symptomatic patients older than 80 years: experience in a single UK centre. Heart 1999;82:13842.

Goldberg RJ, Larson M, Levy D. Factors associated with survival to 75 years of age in middle-aged men and women. The Framingham Study. Arch Intern Med 1996;156(5):505-9.

Goldin JG, Yoon HC, Greaser LE, 3rd, et al. Spiral versus electron-beam CT for coronary artery calcium scoring. Radiology 2001;221(1):213-21. 
Grace AA, Brooks NH, Schofield PM. Beneficial effects of angiotensin converting enzyme inhibitors in severe symptomatic aortic stenosis. Eur Heart $J$ 1991;12(Suppl):129 (Abstract).

Gunduz H, Akdemir R, Binak E, Tamer A, Keser N, Uyan C. Can serum lipid and CRP levels predict the "severity" of aortic valve stenosis? Acta Cardiol 2003;58(4):321-6.

Haberl R, Becker A, Leber A, et al. Correlation of coronary calcification and angiographically documented stenoses in patients with suspected coronary artery disease: results of 1,764 patients. J Am Coll Cardiol 2001;37:451-7.

Hartmann F, Packer M, Coats AJ, et al. Prognostic impact of plasma N-terminal probrain natriuretic peptide in severe chronic congestive heart failure: a substudy of the Carvedilol Prospective Randomized Cumulative Survival (COPERNICUS) trial. Circulation 2004;110(13):1780-6.

Hayashi T, Nakayama Y, Tsumura K, et al. Reflection in the arterial system and the risk of coronary heart disease. Am J Hypertens 2002;15:405-9.

Hecht HS, Harman SM. Relation of aggressiveness of lipid-lowering treatment to changes in calcified plaque burden by electron beam tomography. Am J Cardiol 2003;92(3):334-6.

Helske S, Lindstedt KA, Laine M, et al. Induction of local angiotensin II-producing systems in stenotic aortic valves. J Am Coll Cardiol 2004;44:1859-66

Hirsch D, Azoury R, Sarig S, Kruth HS. Colocalization of cholesterol and hydroxyapatite in human atherosclerotic lesions. Calcif Tissue Int 1993;52:94-8.

Hong C, Becker CR, Schoepf UJ, Ohnesorge B, Bruening R, Reiser MF. Coronary artery calcium: absolute quantification in nonenhanced and contrast-enhanced multidetector row CT studies. Radiology 2002;223(2):474-80

Houslay ES, Cowell SJ, Prescott RJ, et al. Scottish Aortic Stenosis and Lipid Lowering Therapy, Impact on Regression Trial Investigators. Progressive coronary calcification despite intensive lipid-lowering treatment: a randomised controlled trial. Heart 2006;92(9):1207-12.

Hultgren HN. Osteitis Deformans (Paget's Disease) and calcific disease of the heart valves. Am J Cardiol 1998;81:1461-4.

Iivanainen AM, Lindroos M, Tilvis R, Heikkila, Kupari M. Natural history of aortic valve stenosis of varying severity in the elderly. Am J Cardiol 1996;78:97-101.

Jialal I, Stein D, Balis D, Grundy SM, Adams-Huet B, Devaraj S. Effect of hydroxymethyl glutaryl coenzyme a reductase inhibitor therapy on high sensitive Creactive protein levels. Circulation 2001;103(15):1933-5. 
Jones P, Kafonek S, Laurora I, Hunninghake D. Comparative dose efficacy study of atorvastatin versus simvastatin, pravastatin, lovastatin, and fluvastatin in patients with hypercholesterolemia (the CURVES study). Am J Cardiol 1998;81:582-7.

Jukema JW, Bruschke AV, van Boven AJ, et al. Effects of lipid lowering by pravastatin on progression and regression of coronary artery disease in symptomatic men with normal to moderately elevated serum cholesterol levels. The Regression Growth Evaluation Statin Study (REGRESS). Circulation 1995;91:2528-40.

Keller C, Schmitz H, Theisen K, Zollner N. Regression of valvular aortic stenosis due to homozygous familial hypercholesterolaemia following plasmapheresis. Klin Wochenschrift 1986;64:338-41.

Kizer JR, Gefter WB, deLemos AS, Scoll BJ, Wolfe ML, Mohler ER 3rd. Electron beam computed tomography for the quantification of aortic valvular calcification. $J$ Heart Valve Dis 2001;10(3):361-6.

Kockx MM, Herman AG. Apoptosis in atherogenesis: implications for plaque destabilization. Eur Heart J 1998;19(Suppl G):G23-G28.

Kohl P, Kerzmann A, Lahaye L, Gerard P, Limet R. Cardiac surgery in octogenarians. Peri-operative outcome and long-term results. Eur Heart $J$ 2001;22:1235-43.

Koos R, Mahnken AH, Sinha AM, Wildberger JE, Hoffmann R, Kuhl HP. Preliminary experience in the assessment of aortic valve calcification by ECG-gated multislice spiral computed tomography. Int J Cardiol 2005;102(2):195-200.

Kvidal P, Bergström R, Hörte L, Ståhle E. Observed and relative survival after aortic valve replacement. J Am Coll Cardiol 2000;35:747-56.

Laurent S, Caviezel B, Beck L, et al. Carotid artery distensibility and distending pressure in hypertensive humans. Hypertension 1994;23(6 Pt 2):878-83.

Laurent S, Boutouyrie P, Asmar R, et al. Aortic stiffness is an independent predictor of all-cause and cardiovascular mortality in hypertensive patients. Hypertension 2001;37(5):1236-41.

Lewis NP, Henderson AH. Calcific aortic stenosis in twins: a clue to its pathogenesis? Eur Heart $J$ 1990;11:90-1.

Lewis SJ, Moye LA, Sacks FM, et al. Effect of pravastatin on cardiovascular events in older patients with myocardial infarction and cholesterol levels in the average range. Results of the Cholesterol and Recurrent Events (CARE) trial. Ann Intern Med 1998;129(9):681-9.

Libman E. Some general considerations concerning the affectations of the valves of the heart. M Clin North America 1917;1:573. 
Lim P, Monin JL, Monchi M, et al. Predictors of outcome in patients with severe aortic stenosis and normal left ventricular function: role of B-type natriuretic peptide. Eur Heart J 2004;25(22):2048-53.

Linblom D, Lindblom U, Qvist J, Lundström H.. Long-term survival rates after heart valve replacement. J Am Coll Cardiol 1990;15:566-73.

Lindroos M, Kupari M, Heikkila J, Tilvis R. Prevalence of aortic valve abnormalities in the elderly: an echocardiographic study of a random population sample. J Am Coll Cardiol 1993;21:1220-5.

Lippert JA, White CS, Mason AC, Plotnick GD. Calcification of aortic valve detected incidentally on CT scans: prevalence and clinical significance. Am J Roentgenol 1995;164:73-7.

Litwin SE, Katz SE, Weinberg EO, Lorell BH, Aurigemma GP, Douglas PS. Serial echocardiographic-Doppler assessment of left ventricular geometry and function in rats with pressure-overload hypertrophy. Chronic angiotensin-converting enzyme inhibition attenuates the transition to heart failure. Circulation 1995;91(10):2642-54.

Liu PY, Tsai WC, Lin CC, Hsu CH, Haung YY, Chen JH. Invasive measurements of pulse wave velocity correlate with the degree of aortic valve calcification and severity associated with matrix metalloproteinases in elderly patients with aortic valve stenosis. Clin Sci (Lond) 2004;107(4):415-22.

Lombard JT, Selzer A. Valvular aortic stenosis. A clinical and haemodynamic profile of patients. Ann Intern Med 1987;106:292-8.

London GM, Blacher J, Pannier B, Guerin AP, Marchais SJ, Safar ME. Arterial wave reflections and survival in end-stage renal failure. Hypertension 2001;38(3):434-8.

Lubien E, DeMaria A, Krishnaswamy P, et al. Utility of B-natriuretic peptide in detecting diastolic dysfunction: comparison with Doppler velocity recordings. Circulation 2002;105(5):595-601.

MacMillan RM, Rees MR, Lumia FJ, Maranhao V. Preliminary experience in the use of ultrafast computed tomography to diagnose aortic valve stenosis. Am Heart $J$ 1988;115(3):665-71.

Maher ER, Young G, Smyth-Walsh B, Pugh S, Curtis JR. Aortic and mitral valve calcification in patients with end-stage renal disease. Lancet 1987;2(8564):875-7.

Mahmud A, Feely J. Effect of smoking on arterial stiffness and pulse pressure amplification. Hypertension 2003;41(1):183-7.

Martinez-Sanchez C, Henne O, Arceo A, et al. Hemodynamic effects of oral captopril in patients with severe aortic stenosis. Arch Inst Cardiol Mex 1996;66:32230 . 
Mautner GC, Roberts WC. Reported frequency of coronary arterial narrowing by angiogram in patients with valvular aortic stenosis. Am J Cardiol 1992;70:539-40.

Mautner GC, Mautner SL, Froehlich J, et al. Coronary artery calcification: assessment with electron beam CT and histomorphometric correlation. Radiology 1994;192:619-23.

McDonagh TA, Cunningham AD, Morrison CE, et al. Left ventricular dysfunction, natriuretic peptides, and mortality in an urban population. Heart 2001;86(1):21-6.

Mensah GA, Friesinger GC. Calcific aortic stenosis and the congenitally bicuspid aortic valve: Did Osler miss the link? Am J Cardiol 1996;77:417-9.

Messika-Zeitoun D, Aubry MC, Detaint D, et al. Evaluation and clinical implications of aortic valve calcification measured by electron-beam computed tomography. Circulation 2004;110(3):356-62.

Mintz GS, Popma JJ, Pichard AD, et al. Patterns of calcification in coronary artery disease. A statistical analysis of intravascular ultrasound and coronary angiography in 1155 lesions. Circulation 1995;91(7):1959-65.

Mitchell GF, Parise H, Benjamin EJ, et al. Changes in arterial stiffness and wave reflection with advancing age in healthy men and women: the Framingham Heart Study. Hypertension 2004;43(6):1239-45.

Mohler ER, Adam LP, McClelland P, Graham L, Hathaway DR. Detection of osteopontin in calcified human aortic valves. Arterioscler Thromb Vasc Biol 1997; 17:547-52.

Mohler ER, Chawla MK, Chang AW, et al. Identification and characterization of calcifying valve cells from human and canine aortic valves. J Heart Valve Dis 1999;8:254-60.

Mohler ER $3^{\text {rd }}$, Gannon F, Reynolds C, Zimmerman R, Keane MG, Kaplan FS. Bone formation and inflammation in cardiac valves. Circulation 2001;103:1522-8.

Mohler ER. Are atherosclerotic processes involved in aortic valve calcification? Lancet 2000;356:524-5.

Monckeburg JG. Der normale histologische Bau and die Sklerose der Aortenklappen. Virchows Arch Pathol Anat Physiol 1904;176:472-514.

Morgan-Hughes GJ, Owens PE, Roobottom CA, Marshall AJ. Three dimensional volume quantification of aortic valve calcification using multislice computed tomography. Heart 2003;89(10):1191-4.

Müller AM, Cronen C, Kupferwasser LI, Oelert H, Müller K, Kirkpatrick CJ. Expression of endothelial cell adhesion molecules on heart valves: up-regulation in degeneration as well as acute endocarditis. J Pathol 2000;191:54-60. 
Myreng Y, Molstad P, Endresen K, Ihlen H. Reproducibility of echocardiographic estimates of the area of stenosed aortic valves using the continuity equation. Int $J$ Cardiol 1990;26(3):349-54.

Nakamura M, Endo H, Nasu M, Arakawa N, Segawa T, Hiramori K. Value of plasma B type natriuretic peptide measurement for heart disease screening in a Japanese population. Heart 2002;87(2):131-5.

Nassimiha D, Aronow WS, Ahn C, Goldman ME. Association of coronary risk factors with progression of valvular aortic stenosis in older persons. Am J Cardiol 2001;87:1313-4.

Newby DE, Cowell SJ, Boon NA. Emerging medical treatments for aortic stenosis: statins, angiotensin converting enzyme inhibitors, or both? Heart 2006;92(6):729-34.

Ngo MV, Gottdiener JS, Fletcher RD, Fernicola DJ, Gersh BJ. Smoking and obesity are associated with the progression of aortic stenosis. Am J Geriatr Cardiol 2001;10:86-90.

Nieman K, Oudkerk M, Rensing BJ, et al. Coronary angiography with multi-slice computed tomography. Lancet 2001;357(9256):599-603.

Nijhuis RL, Hofman A, Witteman JC. C-reactive protein predicts progression of atherosclerosis measured at various sites in the arterial tree: the Rotterdam Study. Stroke 2002;33(12):2750-5.

Novaro GM, Tiong IY, Pearce GL, Lauer MS, Sprecher DL, Griffin BP. Effect of hydroxymethylglutaryl Coenzyme A reductase inhibitors on the progression of calcific aortic stenosis. Circulation 2001;104:2205-9.

O'Brien KD, Kuusisto J, Reichenbach DD, et al. Osteopontin is expressed in human aortic valvular lesions. Circulation 1995;92:2163-8.

O'Brien KD, Reichenbach DD, Marcovina SM, Kuusisto J, Alpers CE, Otto CM. Apolipoprotein B, (a), and E accumulate in the morphologically early lesion of 'degenerative' valvular aortic stenosis. Arterioscler Thromb Vasc Biol 1996;16:52332 .

O'Brien KD, Zhao XQ, Shavelle DM, et al. Hemodynamic effects of the angiotensinconverting enzyme inhibitor, ramipril, in patients with mild to moderate aortic stenosis and preserved left ventricular function. J Investig Med 2004;52(3):185-91.

O'Brien KD, Probstfield JL, Caulfield MT, et al. Angiotensin-converting enzyme inhibitors and change in aortic valve calcium. Arch Intern Med. 2005;165:858-62.

O'Keefe JH Jr, Vlietstra RE, Bailey KR, Holmes DR Jr. Natural history of candidates for balloon aortic valvuloplasty. Mayo Clin Proc 1987;62:986-91. 
Olsson M, Granström L, Lindblom D, Rosenquist M, Rydén L. Aortic valve replacement in octogenarians with aortic stenosis: a case control study. $\mathrm{J} \mathrm{Am} \mathrm{Coll}$ Cardiol 1992;20:1512-6.

Olsson M, Dalsgaard C, Haegerstrand A, Rosenqvist M, Rydén L, Nilsson J. Accumulation of $\mathrm{T}$ lymphocytes and expression of interleukin-2 receptors in nonrheumatic stenotic aortic valves. J Am Coll Cardiol 1994;23:1162-70.

Olsson M, Janfjäll H, Orth-Gomért K, Undén A, Rosenqvist M. Quality of life in octogenarians after valve replacement due to aortic stenosis. Eur Heart $J$ 1996;17:583-9.

Olsson M, Thyberg J, Nilsson J. Presence of oxidised low density lipoprotein in nonrheumatic stenotic aortic valves. Arterioscler Thromb Vasc Biol 1999;19:121822.

Ortlepp JR, Hoffmann R, Ohme F, Lauscher J, Bleckmann F, Hanrath P. The vitamin $\mathrm{D}$ receptor genotype predisposes to the development of calcific aortic valve stenosis. Heart 2001;85:635-8.

Osler W. The bicuspid condition of the aortic valves. Trans Assoc Am Phys 1886;1:185-92.

Otto CM, Kuusisto J, Reichenbach DD, Gown AM, O'Brien KD. Characterization of the early lesion of 'degenerative' valvular aortic stenosis: histologic and immunohistochemical studies. Circulation 1994;90:844-53.

Otto CM, Burwash IG, Legget ME, et al. Prospective study of asymptomatic valvular aortic stenosis. Clinical, echocardiographic, and exercise predictors of outcome. Circulation 1997;95:2262-70.

Otto CM, Lind BK, Kitzman DW, Gersch BJ, Siscovick DS. Association of aorticvalve sclerosis with cardiovascular mortality and morbidity in the elderly. $N$ Engl J Med 1999;341:142-7.

Otto CM, O' Brien KD. Why is there discordance between calcific aortic stenosis and coronary artery disease? Heart 2001;85:601-2.

Otto CM. Calcification of bicuspid aortic valves. Heart 2002;88:321-2.

Otto CM. Valvular aortic stenosis: disease severity and timing of intervention. $J \mathrm{Am}$ Coll Cardiol 2006;47(11):2141-51.

Palta S, Pai AM, Gill KS, Pai RG. New insights into the progression of aortic stenosis. Implications for secondary prevention. Circulation 2000;101:2497-502.

Parhami F, Morrow AD, Balucan J, et al. Lipid oxidation products have opposite effects on calcifiying vascular cell and bone cell differentiation. A possible explanation for the paradox of arterial calcification in osteoporotic patients. Arterioscler Thromb Vasc Biol 1997;17:680-7. 
Pearlman AS. Medical treatment of aortic stenosis. Promising, or wishful thinking? $J$ Am Coll Cardiol 2002;40:1731-4.

Pellikka PA, Nishimura RA, Bailey KR, Tajik AJ. The natural history of adults with asymptomatic, hemodynamically significant aortic stenosis. $J$ Am Coll Cardiol 1990;15:1012-7.

Peltier M, Trojette F, Sarano ME, Grigioni F, Slama MA, Tribouilloy CM. Relation between cardiovascular risk factors and nonrheumatic severe calcific aortic stenosis among patients with a three-cuspid aortic valve. Am J Cardiol 2003;91:97-9.

Peter M, Hoffman A, Parker C, Luescher T, Burckhardt D. Progression of aortic stenosis. Role of age and concomitant coronary artery disease. Chest 1993;103:17159.

Pitt B, Mancini GB, Ellis SG, Rosman HS, Park JS, McGovern ME. Pravastatin limitation of atherosclerosis in the coronary arteries (PLAC I): reduction in atherosclerosis progression and clinical events. PLAC I investigation. J Am Coll Cardiol 1995;26:1133-9.

Pohle K, Maeffert R, Ropers D, et al. Progression of aortic valve calcification. Association with coronary atherosclerosis and cardiovascular risk factors. Circulation 2001;104(16):1927-32.

Pomerance A. Pathogenesis of aortic stenosis and its relation to age. Br Heart $J$ 1972;34:569-74.

Prockop DJ. Marrow stromal cells as stem cells for nonhematopoietic tissues. Science 1997;276:71-4.

Qanadli SD, Mesurolle B, Aegerter P, et al. Volumetric quantification of coronary artery calcifications using dual-slice spiral CT scanner: improved reproducibility of measurements with 180 degrees linear interpolation algorithm. J Comput Assist Tomogr 2001;25(2):278-86.

Raggi P, Cooil B, Shaw LJ, et al. Progression of coronary calcium on serial electron beam tomographic scanning is greater in patients with future myocardial infarction. Am J Cardiol 2003;92(7):827-9.

Raggi P, Davidson M, Callister TQ, et al. Aggressive versus moderate lipid-lowering therapy in hypercholesterolemic postmenopausal women: Beyond Endorsed Lipid Lowering with EBT Scanning (BELLES). Circulation 2005;112(4):563-71.

Rajamannan NM. Calcific aortic stenosis: a disease ready for prime time. Circulation 2006;114(19):2007-9.

Rajavashisth TB, Andalibi A, Territo MC, et al. Induction of endothelial cell expression of granulocyte and macrophage colony-stimulating factors by modified low density lipoproteins. Nature 1990;344:254-7. 
Rallidis L, Naoumova RP, Thompson GR, Nihoyannopoulos P. Extent and severity of atherosclerotic involvement of the aortic valve and root in familial hypercholesterolaemia. Heart 1998;80:583-90.

Rapp AH, Hillis LD, Lange RA, Cigarroa JE. Prevalence of coronary artery disease in patients with aortic stenosis with and without angina pectoris. Am $J$ Cardiol 2001;87:1216-7.

Ridker PM, Glynn RJ, Hennekens CH. C-reactive protein adds to the predictive value of total and HDL cholesterol in determining risk of first myocardial infarction. Circulation 1998;97(20):2007-11.

Roberts WC. The senile cardiac calcification syndrome. Am J Cardiol 1986;58:5724.

Robicsek F, Harbold NBJ, Daugherty HK, et al. Ballon valvuloplasty in calcified aortic stenosis: a cause for caution and alarm. Ann Thorac Surg 1988;45:515-25.

Roger VL, Tajik AJ, Bailey KR, Oh JK, Taylor CL, Seward JB. Progression of aortic stenosis in adults: New appraisal using Doppler echocardiography. Am Heart $J$ 1990;119:331-8.

Rosenhek R, Binder T, Porenta G, et al. Predictors of outcome in severe, asymptomatic aortic stenosis. New Engl J Med 2000;343:611-7.

Rosenhek R, Klaar U, Schemper M, et al. Mild and moderate aortic stenosis. Natural history and risk stratification by echocardiography. Eur Heart $J$ 2004a;25(3):199205.

Rosenhek R, Rader F, Loho N, et al. Statins but not angiotensin-converting enzyme inhibitors delay progression of aortic stenosis. Circulation 2004b;110:1291-5.

Ross J, Braunwald E. Aortic stenosis. Circulation 1968;38(1 Suppl):61-7.

Routledge HC, Townend JN. ACE inhibition in aortic stenosis: dangerous medicine or golden opportunity? J Hum Hyperten 2001;15(10):659-67.

Rumberger JA, Simons DB, Fitzpatrick LA, Sheedy PF, Schwartz RS. Coronary artery calcium area by electron-beam computed tomography and coronary atherosclerotic plaque area. A histopathologic correlative study. Circulation 1995;92(8):2157-62.

Rumberger JA, Kaufman L. A rosetta stone for coronary calcium risk stratification: agatston volume, and mass scores in 11,490 individuals. Am J Roentgenol 2003:181(3):743-8.

Salomaa V, Riley W, Kark JD, Nardo C, Folsom AR. Non-insulin-dependent diabetes mellitus and fasting glucose and insulin concentrations are associated with arterial stiffness indexes. The ARIC Study. Atherosclerosis Risk in Communities Study. Circulation 1995;91(5):1432-43. 
Samal AK, Berman AE, Kuruvanka TS, Nasca PF, Ventura HO, Gilliand YE. Effect of statin therapy in the progression of moderate to severe calcific aortic stenosis. Circulation 2002;106 (Suppl II):II-640.

Sanchez PL, Santos JL, Kaski JC, et al. Relation of circulating C-reactive protein to progression of aortic valve stenosis. Am J Cardiol 2006;97(1):90-3.

Sangiorgi G, Rumberger JA, Severson A, et al. Arterial calcification and not lumen stenosis is highly correlated with atherosclerotic plaque burden in humans: a histologic study of 723 coronary artery segments using nondecalcifying methodology. J Am Coll Cardiol 1998;31(1):126-33.

Sarig S, Weiss TA, Katz I, et al. Detection of cholesterol associated with calcium mineral using confocal fluorescence microscopy. Lab Invest 1994;71:782-7.

Schmermund A, Baumgart D, Mohlenkamp S, et al. Natural history and topographic pattern of progression of coronary calcification in symptomatic patients: An electronbeam CT study. Arterioscler Thromb Vasc Bio 2001;21(3):421-6.

Schwartz GG, Oliver MF, Ezekowitz MD, et al. Rationale and design of the Myocardial Ischemia Reduction with Aggressive Cholesterol Lowering (MIRACL) study that evaluates atorvastatin in unstable angina pectoris and in non-Q-wave acute myocardial infarction. Am J Cardiol 1998;81(5):578-81.

Sharony R, Grossi EA, Saunders PC, et al. Aortic valve replacement in patients with impaired ventricular function. Ann Thorac Surg 2003;75:1808-14.

Shavelle DM, Takasu J, Budoff MJ, Mao S, Zhao X, O'Brien K. HMG CoA reductase inhibitor (statin) and aortic valve calcium. Lancet 2002;359:1125-6.

Shemesh J, Apter S, Rozenman J, et al. Calcification of coronary arteries: detection and quantification with double-helix CT. Radiology 1995;197: 779-83.

Shepherd J, Cobbe SM, Ford I, et al. Prevention of coronary heart disease with pravastatin in men with hypercholesterolaemia. N Engl J Med 1995;333:1301-7.

Skowasch D, Schrempf S, Preusse CJ, et al. Tissue resident C reactive protein in degenerative aortic valves: correlation with serum $\mathrm{C}$ reactive protein concentrations and modification by statins. Heart 2006;92:495-8.

Sprigings DC, Forfar JC. How should we manage symptomatic aortic stenosis in the patient who is 80 or older? Br Heart J 1995;74:481-4.

Stary HC. The development of calcium deposits in atherosclerotic lesions and their persistence after lipid regression. Am J Cardiol 2001;88(2A):16E-19E.

Stewart BF, Siscovick D, Lind BK, et al for the Cardiovascular Health Study. Clinical factors associated with calcific aortic valve disease. J Am Coll Cardiol 1997;29:630-4. 
Straumann, Meyer B, Misteli M, Blumberg A, Jenzer HR. Aortic and mitral valve disease in patients with end stage renal failure on long-term haemodialysis. Br Heart $J$ 1992;67:236-9.

Sundt TM, Bailey MS, Moon MR, et al. Quality of life after aortic valve replacement at the age of $>80$ years. Circulation 2000;102 (Suppl III):III70-74.

Tentolouris C, Kontozoglou T, Toutouzas P. Familial calcification of aorta and calcific aortic valve disease associated with immunologic abnormalities. Am Heart $J$ 1993;126:904-9.

The Heart Protection Study Collaborative Group. MRC/BHF Heart Protection Study of cholesterol lowering with simvastatin in 20,536 high-risk individuals: a randomised placebo controlled trial. Lancet 2002;360:7-22.

The Long-term Intervention with Pravastatin in Ischaemic Disease (LIPID) Study Group. Prevention of cardiovascular events and death with pravastatin in patients with coronary heart disease and a broad range of initial cholesterol levels. $N$ Engl $J$ Med 1998;339(19):1349-57.

The Scandinavian Simvastatin Survival Study (4S). Randomised trial of cholesterol lowering in 4444 patients with coronary heart disease: Lancet 1994;344(8934):13839 .

Thubrikar MJ, Aouad J, Jolan SP. Patterns of calcific deposits in operatively excised stenotic or purely regurgitant aortic valves and their relation to mechanical stress. $\mathrm{Am}$ J Cardiol 1986;58:304-8.

Treasure T, MacRae KD. Minimisation: the platinum standard for trials? Br Med J 1998;317:362-3.

Tsutamoto T, Wada A, Maeda K, et al. Attenuation of compensation of endogenous cardiac natriuretic peptide system in chronic heart failure: prognostic role of plasma brain natriuretic peptide concentration in patients with chronic symptomatic left ventricular dysfunction. Circulation 1997;96(2):509-16.

Turcani M, Rupp H. Heart failure development in rats with ascending aortic constriction and angiotensin-converting enzyme inhibition. $\mathrm{Br} J$ Pharmacol 2000;130(7):1671-7.

Umana E, Ahmed W, Alpert MA. Valvular and perivalvular abnormalities in endstage renal disease. Am J Med Sci 2003;325:237-42.

Van Der Meer IM, De Maat MP, et al. C-reactive protein predicts progression of atherosclerosis measured at various sites in the arterial tree: the Rotterdam Study. Stroke 2002;33(12):2750-5.

van Popele NM, Grobbee DE, Bots ML, et al. Association between arterial stiffness and atherosclerosis: the Rotterdam Study. Stroke 2001;32(2):454-60. 
Vandeplas A, Willems JL, Piessens J, De Geest H. Frequency of angina pectoris and coronary artery disease in severe isolated valvular aortic stenosis. Am J Cardiol 1988;62:117-20.

Wagner S, Selzer A. Patterns of progression of aortic stenosis: a longitudinal hemodynamic study. Circulation 1982;65: 709-12.

Wallby L, Janerot-Sjöberg B, Steffensen T, Broqvist M. T lymphocyte infiltration in non-rheumatic aortic stenosis: a comparative descriptive study between tricuspid and bicuspid aortic valves. Heart 2002;88:348-51.

Ward C. Clinical significance of the bicuspid aortic valve. Heart 2000;83:81-5.

Weber T, Auer J, O'Rourke MF, et al. Arterial stiffness, wave reflections, and the risk of coronary artery disease. Circulation 2004;109(2):184-9.

Weber T, Auer J, O'rourke MF, et al. Increased arterial wave reflections predict severe cardiovascular events in patients undergoing percutaneous coronary interventions. Eur Heart $J$ 2005;26(24):2657-63.

Weinberg EO, Schoen FJ, George D, et al. Angiotensin-converting enzyme inhibition prolongs survival and modifies the transition to heart failure in rats with pressure overload hypertrophy due to ascending aortic stenosis. Circulation 1994;90(3):1410-22.

Wilkinson IB, Prasad K, Hall IR, et al. Increased central pulse pressure and augmentation index in subjects with hypercholesterolemia. J Am Coll Cardiol 2002;39(6):1005-11.

Willmann JK, Weishaupt D, Lachat M, et al. Electrocardiographically gated multidetector row CT for assessment of valvular morphology and calcification in aortic stenosis. Radiology 2002;225(1):120-8.

Wilmshurst PT, Stevenson RN, Griffiths H, Lord JR. A case control investigation of the relation between hyperlipidaemia and calcific aortic valve stenosis. Heart 1997;78:475-9.

Wongpraparut N, Apiyasawat S, Crespo G, Yazdani K, Jacobs LE, Kotler MN. Determinants of progression of aortic stenosis in patients aged $\geq 40$ years. Am J Cardiol 2002;89:350-2.

Woodring JH, West JW. CT of aortic and mitral valve calcification. J Ky Med Assoc 1989;87:177-80.

Yearwood TL, Misbach GA, Chandran KB. Experimental fluid dynamics of aortic stenosis in a model of the human aorta. Clin Phys Physiol Meas 1989;10:11-24.

Zhao XQ, Brown BG, Hillger L, et al. Effects of intensive lipid-lowering therapy on the coronary arteries of asymptomatic subjects with elevated apolipoprotein B. Circulation 1993;88:2744-53. 
Zoghbi WA, Enriquez-Sarano M, Foster E, et al for the American Society of Echocardiography. Recommendations for evaluation of the severity of native valvular regurgitation with two-dimensional and Doppler echocardiography. $\mathrm{J} \mathrm{Am}$ Soc Echocardiogr 2003;16:777-802. 


\section{PUBLICATIONS}

Cowell SJ, Newby DE, Burton J, White A, Northridge DB, Boon NA, Reid J. Aortic Valve Calcification On Computed Tomography Predicts The Severity Of Aortic Stenosis. Clin Radiol 2003;58:712-6.

Cowell SJ, Newby DE, Boon NA, Elder AT. Calcific aortic stenosis: Same old story? Age and Ageing 2004;33:538-44.

Cowell SJ, Newby DE, Prescott RJ, Bloomfield P, Reid J, Northridge DB, Boon NA. Scottish Aortic stenosis and Lipid-lowering Trial, Impact on REgression (SALTIRE) Investigators. A randomised trial of intensive lipid-lowering therapy in calcific aortic stenosis. N Engl J Med 2005;352:2389-97.

Houslay E, Cowell SJ, Prescott R, Reid J, Burton J, Northridge DB, Boon NA, Newby DE. Progressive coronary calcification despite intensive lipid-lowering therapy: a randomised controlled trial. Heart 2006;92:1207-12.

Newby DE, Cowell SJ, Boon NA. Emerging medical treatments for aortic stenosis: statins, angiotensin- converting enzyme inhibitors, or both? Heart 2006;92:729-34. 


\title{
Aortic Valve Calcification on Computed Tomography Predicts the Severity of Aortic Stenosis
}

\author{
S. J. COWELL*, D. E. NEWBY*, J. BURTON†, A. WHITE $\ddagger$, D. B. NORTHRIDGE $\ddagger$, \\ N. A. BOON ${ }^{*}$, J. REIDt \\ Departments of *Cardiology, Royal Infirmary, Edinburgh, $\dagger$ Radiology, Borders General Hospital, Roxburghshire, \\ and $\ddagger$ Cardiology, Western General Hospital, Edinburgh, Scotland, UK
}

Received: 18 September 2002 Revised: 26 February 2003 Accepted: 4 March 2003

\begin{abstract}
AIM: Incidental aortic valve calcification is often detected during computed tomography. The aim was to compare the severity of valvular stenosis and calcification in patients with aortic stenosis. MATERIALS AND METHODS: One hundred and fifty-seven patients aged $68 \pm 11$ years (range: 34-85) with aortic valve stenosis underwent multislice helical computed tomography and Doppler echocardiography performed by independent, blinded observers. The aortic valve calcium score was determined using automated computer software calibrated with a phantom.

RESULTS: Doppler echocardiography demonstrated a post-valve velocity of $3.45 \pm 0.66 \mathrm{~m} / \mathrm{s}$ and a peak gradient of $49 \pm 11 \mathrm{mmHg}$. Computed tomography showed excellent reproducibility and the median aortic valve calcium score was $5858 \mathrm{AU}$ (interquartile range, 1555-14,596). The computed tomography aortic valve calcium score positively correlated with the Doppler post-valve velocity and peak gradient $(r=0.54, p<0.0001$ for both) of the aortic valve. All patients with severe aortic stenosis had a calcium score of $>3700 \mathrm{AU}$.

CONCLUSION: Calcification of the aortic valve is closely associated with the severity of aortic stenosis, and heavy calcification suggests the presence of severe aortic stenosis that requires urgent cardiological assessment. Patients with lesser degrees of aortic valve calcification should be screened for aortic stenosis and monitored for disease progression. Cowell, S. J. et al. (2003). Clinical Radiology 58: 712-716.
\end{abstract}

(C) 2003 Published by Elsevier Science Ltd on behalf of The Royal College of Radiologists.

Key words: calcification, aortic valve, computed tomography, echocardiography.

\section{INTRODUCTION}

Calcific aortic stenosis is the commonest reason for valve replacement in the developed world. The condition may be due to progressive calcification of a congenitally bicuspid valve or "degenerative" calcification of a morphologically normal valve [1]. Irrespective of the aortic valve morphology, the histological features are surprisingly similar to those of coronary atheroma and include lipid deposition, fibrosis and calcification [2].

The rate of progression of aortic stenosis appears to be most rapid in those patients with severe calcific disease $[3,4]$. The annual increase in aortic valve gradient is more than twice that seen in patients with non-calcific disease $(9.7$ versus

Guarantor and correspondent: Dr S. J. Cowell, Department of Cardiology, Royal Infirmary, Little France Crescent, Old Dalkeith Rd, Edinburgh, EH16 4SU, Scotland, UK. Tel: +44-131-242-1852; Fax: +44131-242-6422; E-mail: jo.cowell@ed.ac.uk
$4.4 \mathrm{mmHg} /$ year) and the grade of calcification correlates with the rate of disease progression [5]. Indeed, moderate or severe aortic valve calcification is the strongest independent risk factor for an adverse clinical outcome with a five-fold increase in the rate of death or aortic valve replacement [4].

Computed tomography is being increasingly used as a noninvasive method of screening for atherosclerotic coronary artery disease [6,7] with $80-100 \%$ sensitivity and $80 \%$ specificity [8]. There is an association between coronary artery disease and calcific aortic stenosis, with approximately a third of patients with aortic stenosis having significant coronary stenoses on angiography [9]. As a consequence, there have been several reports of incidental aortic valve calcification detected during computed tomography examinations with a prevalence of $10-30 \%[10,11]$. However, there have been few reports [11] assessing the relationship between the degree of valvular calcification and the severity of aortic stenosis. We hypothesized that valvular calcification would correlate with the aortic post-valve velocity in patients with aortic stenosis. 


\section{MATERIALS AND METHODS}

\section{Subjects}

One hundred and fifty-seven patients participated in the study, which was undertaken with the approval of the local research ethics committee, in accordance with the Declaration of Helsinki, and with the written informed consent of each participant. This is a sub-study of the Scottish Aortic stenosis and Lipid-lowering Therapy, Impact on REgression (SALTIRE) trial that is evaluating the effect of lipid lowering therapy on the rate of progression of aortic stenosis. Patients with aortic stenosis were approached for inclusion in the trial if they were older than 18 years and had a peak aortic valve velocity of $\geq 2.5 \mathrm{~m} / \mathrm{s}$ on Doppler echocardiography. Patients were excluded if they were women of child-bearing potential, had active liver disease, were planned to receive or were receiving lipid-lowering therapy, had severe mitral valve disease, aortic regurgitation or planned valve replacement. One patient, with a previous aortic root abscess, was excluded because of extensive aortic root calcification that obscured and prevented assessment of the aortic valve.

All patients underwent both echocardiography and computed tomography within the month before randomization to study therapy (atorvastatin $80 \mathrm{mg}$ daily or placebo). Only pre-intervention baseline data are presented here.

\section{Echocardiography}

The echocardiograms were all performed on an ATL-3000 cardiac ultrasound machine [Philips Medical Systems (UK) Limited, Stevenage, UK] using a $3 \mathrm{MHz}$ transducer for $\mathrm{M}$-mode, and two-dimensional imaging with integral pulsed and continuous wave Doppler. The peak instantaneous aortic valve gradient was determined using the modified Bernoulli equation, and the aortic valve area by the continuity equation. Aortic valve calcification was graded using the Rosenhek classification [4]. A single operator blinded to the results of the computed tomogram performed all echocardiographic examinations and analyses.

\section{Computed Tomography}

The computed tomography was performed using a multislice helical scanner [Twin II Flash; Philips Medical Systems (UK) Limited, Stevenage, UK]. The region of the aortic valve and coronary arteries was assessed using $2.7 \mathrm{~mm}$ slices, with a pitch of 0.7 and an increment of $1.4 \mathrm{~mm}$ during held inspiration. Operators blinded to the results of the echocardiogram performed all examinations and analyses. CT scanner quality assurance was performed before each examination with calibration against a standard phantom. Off-line analysis of the cardiac images was conducted using an automated, computerized software program (Picker Cardiac Scoring). This employs a modified Agatston scoring method [12] that uses a threshold of $90 \mathrm{HU}$ to compensate for non-gated imaging. This modification produces comparable sensitivity and specificity to electron beam CT. Calcium scores were individually calculated for the aortic valve, and all three coronary arteries by summing the lesion scores for all sections containing calcium.

\section{Reproducibility}

Two unselected random samples of 20 patients each were taken from the study population. Subjects underwent repeated computed tomography or echocardiography within 4 weeks of the first examination and before administration of the study medication.

\section{Data Analysis and Statistics}

Data are expressed as mean \pm standard deviation of the mean (SD). The calcium scores were not normally distributed and are expressed as median with interquartile ranges. After calibration with the study phantom, the aortic valve and coronary artery calcium volume scores are expressed as arbitrary units (AU). Reproducibility was assessed by the method of Bland and Altman [13], and expressed as the mean of the differences and the coefficient of repeatability (twice the standard deviation of the differences). As the difference of the two measures was proportional to their mean, the data for the aortic valve calcium score underwent logarithmic transformation [13]. Data were compared using regression analysis and analysis of variance (ANOVA) using StatView version 5.0.1 (SAS Institute Inc., Cary, NC, USA). Where ANOVA demonstrated significant differences in responses, post-hoc comparisons were made using the Fisher's protected least squares difference (PLSD) test (StatView version 5.0.1). Statistical significance was taken at the $5 \%$ level.

\section{RESULTS}

Patient characteristics are listed in Table 1. In keeping with the study population, patients were predominantly male, elderly and had haemodynamically significant aortic stenosis. Both echocardiography and computed tomography showed excellent reproducibility (Table 2 ).

Table 1 - Baseline subject characteristics

\begin{tabular}{lc}
\hline Number & 157 \\
Age (years) & $68 \pm 11$ \\
Sex (male) & $71 \%$ \\
Bicuspid aortic valve & 5 \\
Atrial fibrillation & 11 \\
& \\
Echocardiogram & $1.08 \pm 0.22$ \\
Pre-valve velocity $(\mathrm{m} / \mathrm{s})$ & $3.45 \pm 0.66$ \\
Post-valve velocity $(\mathrm{m} / \mathrm{s})$ & $49 \pm 19$ \\
Peak gradient $(\mathrm{mmHg})$ & $27 \pm 11$ \\
Mean gradient $(\mathrm{mmHg})$ & $1.02 \pm 0.40$ \\
Valve area $\left(\mathrm{cm}^{2}\right)$ & \\
& \\
Computed tomogram & \\
Aortic valve $(\mathrm{AU})$ & $5858(1555-14596)$ \\
Coronary artery $(\mathrm{AU})$ & $97(0-603)$ \\
LAD & $0(0-36)$ \\
Circumflex & $0(0-0)$ \\
Right & $121(0-731)$ \\
Total &
\end{tabular}

Mean $\pm S D ; L A D$, left anterior descending. AU, arbitrary units.

- Median (interquartile range). 
All but two patients had significant aortic valve calcification on computed tomography (Fig. 1). The median aortic valve calcium score was $5858 \mathrm{AU}$. The majority of patients (107/157) had detectable coronary artery calcification that predominantly affected the left anterior descending coronary artery. There was no correlation between the magnitude of the aortic valve and total coronary calcium scores $(r=0.04, p=0.61)$.

\section{Comparison Between Echocardiography and Computed Tomography}

Echocardiographic grade of calcification correlated weakly with the computed tomography aortic valve calcium score $(r=0.29, p<0.001)$ and the peak post-aortic valve velocity $(r=0.40, p<0.001)$. The computed tomography aortic valve calcium score correlated strongly with the post-valve velocity $(r=0.54, p<0.0001: y=0.00004 x+3.09$; Fig. 2) and the mean $(r=0.54, p<0.0001 ; y=0.0008 x+20.7)$ and peak gradient $(r=0.54, p<0.0001: y=0.0013 x+39.1)$ of the aortic valve, but only weakly correlated with the aortic valve area $(r=0.20, p=0.01)$.

Stratifying the patients according to the quintiles of calcification demonstrated a progressive increase in the mean peak post-aortic valve velocity (Fig. 3). All patients with severe aortic valve stenosis (post-valve velocity $>4 \mathrm{~m} / \mathrm{s}$ ) had an aortic valve calcium score of $>3700 \mathrm{AU}$. This threshold gives a sensitivity of $100 \%$ and specificity of $50 \%$ that translates into a negative predictive value of $100 \%$ and a positive predictive value of $39 \%$ for the detection of severe aortic stenosis. A threshold of $6000 \mathrm{AU}$ gives a sensitivity of $90 \%$ and specificity of $66 \%$ giving a negative predictive value of $95 \%$ and a positive predictive value of $45 \%$.

\section{DISCUSSION}

In patients with aortic stenosis, we have demonstrated a close association between the degree of aortic valve calcification and the haemodynamic severity of aortic stenosis. In particular, the presence of severe and potentially critical aortic stenosis is associated with heavy calcification. We suggest that patients found to have incidental aortic valve calcification on computed tomography require further cardiological assessment for aortic stenosis, especially when there is heavy calcification.

This is the first study to compare aortic valve calcium scores with echocardiogram-derived measures of valvular gradients in a large number of patients with aortic stenosis. One previous study of 19 patients also suggested that there may be an association between the severity of aortic stenosis and the valvular calcium score [14]. We have studied a larger population with sufficient power to demonstrate a marked correlation between these parameters. However, because of the selected study population, these findings should only be cautiously extrapolated to aortic valve calcification identified during general population screening or as an incidental finding. A recent retrospective study suggested a $20 \%$ incidence of aortic valve calcification in over 2000 patients attending for detection of coronary calcification [14]. One retrospective study of 109 such patients who had undergone both computed tomography and echocardiography, reported a $30 \%$ prevalence of aortic valve calcification in which aortic stenosis was documented in $15 \%$ [11]. In the absence of aortic valve calcification, none of the patients had significant aortic valve stenosis. In the current study, only two patients $(1 \%)$ had no detectable valvular calcification suggesting an excellent negative predictive value. Our study findings additionally suggest that the likelihood of significant valvular stenosis increases with the severity of calcification.

Echocardiography is the mainstay of clinical monitoring for aortic valve stenosis but provides only a subjective and semiquantitative measure of aortic valve calcification. Computed tomography provides a more accurate method of quantifying calcium that more closely correlates with the aortic valve gradient than echocardiography-derived measures of calcification. It may be useful to quantify more accurately the degree of calcification given that it is the strongest independent risk factor for disease progression and an adverse clinical outcome [4]. Further prospective studies are now needed to assess whether the degree of calcification [15] provides useful additional clinical information that would help guide patient management. Indeed, it has been suggested that patients with severe aortic stenosis and marked calcification should undergo aortic valve replacement even in the absence of symptoms [4].

Aortic valve calcification is associated with an increased cardiovascular mortality [16]. The underlying pathogenetic process appears to share many of the features and risk factors for atherosclerosis [2] including hypercholesterolaemia [17,18] that is associated with a more rapid progression of aortic valve calcification [15]. There are several ongoing studies, including the SALTIRE trial, that are assessing the impact of lipidlowering therapy on the rate of progression of aortic stenosis. Given that statin use is associated with halting the progression of coronary calcification [19], the present study indicates that multislice helical computed tomography is a valuable method

Table 2 - Reproducibility of echocardiographic and computed tomographic assessments of the aortic valve $(n=20)$

\begin{tabular}{|c|c|c|c|c|}
\hline & Mean & Mean of differences & Standard deviation of differences & Cocfficient of repeatability \\
\hline \multicolumn{5}{|l|}{ Echocardiogram } \\
\hline Post-valve velocity $(\mathrm{m} / \mathrm{s})$ & $3.20 \pm 0.11$ & 0.0 & 0.16 & 0.32 \\
\hline Peak gradient $(\mathrm{mmHg})$ & $41.7 \pm 3.0$ & 0.0 & 4.1 & 8.2 \\
\hline Mean gradient $(\mathrm{mmHg})$ & $21.6 \pm 1.8$ & 0.2 & 3.8 & 7.6 \\
\hline Valve Area $\left(\mathrm{cm}^{2}\right)$ & $1.17 \pm 0.10$ & 0.06 & 0.39 & 0.78 \\
\hline \multicolumn{5}{|l|}{ Computed tomography ${ }^{*}$} \\
\hline Valve calcium score (pAU) & $3.86 \pm 0.49$ & 0.01 & 0.04 & 0.07 \\
\hline
\end{tabular}

- Aortic valve calcium score has undergone logarithmic transformation. 


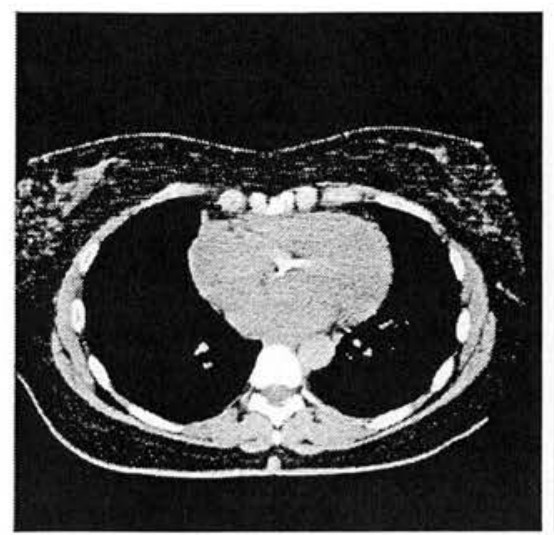

Mild

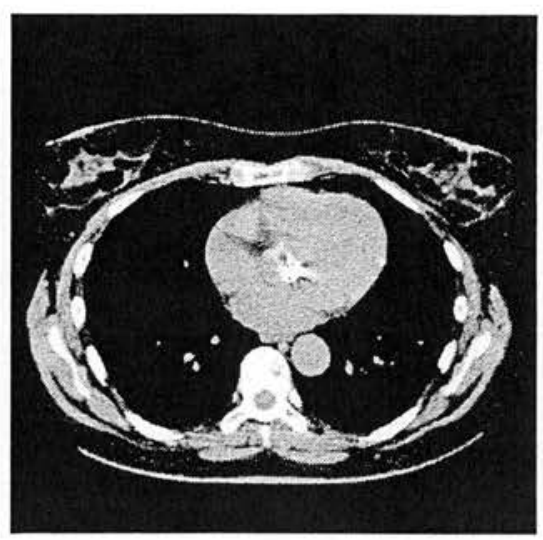

Moderate

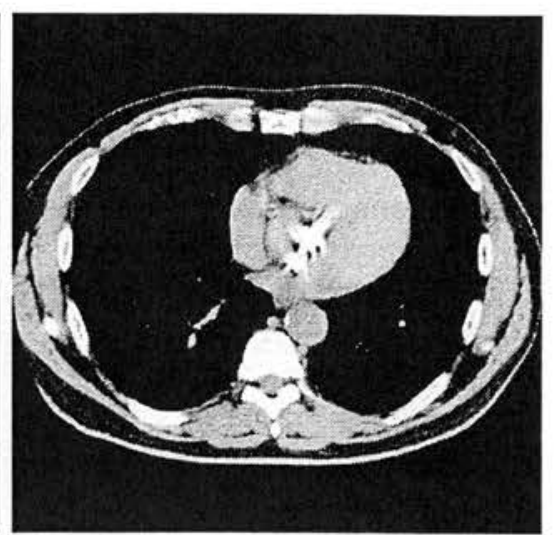

Severe

Fig. 1 - Representative computed tomograms showing mild, moderate and severe calcification of the aortic valve.

of assessing aortic valve calcification and disease progression in such intervention trials. Indeed, one preliminary observational study has suggested that statin use is associated with a lower rate of progression of aortic valve calcification [20].

Using our methodology, we have found a close correlation between echocardiographic measures of aortic stenosis and aortic valve calcium scores measured by multislice helical computed tomography. The applicability of our findings to other CT machines with differing specifications, such as 2,4 or 16 slice acquisitions, or imaging parameters, such as slice thickness and pitch, is unknown. However, there is a high degree of agreement between different machines and coronary calcium scores [21], and we believe that our findings will be

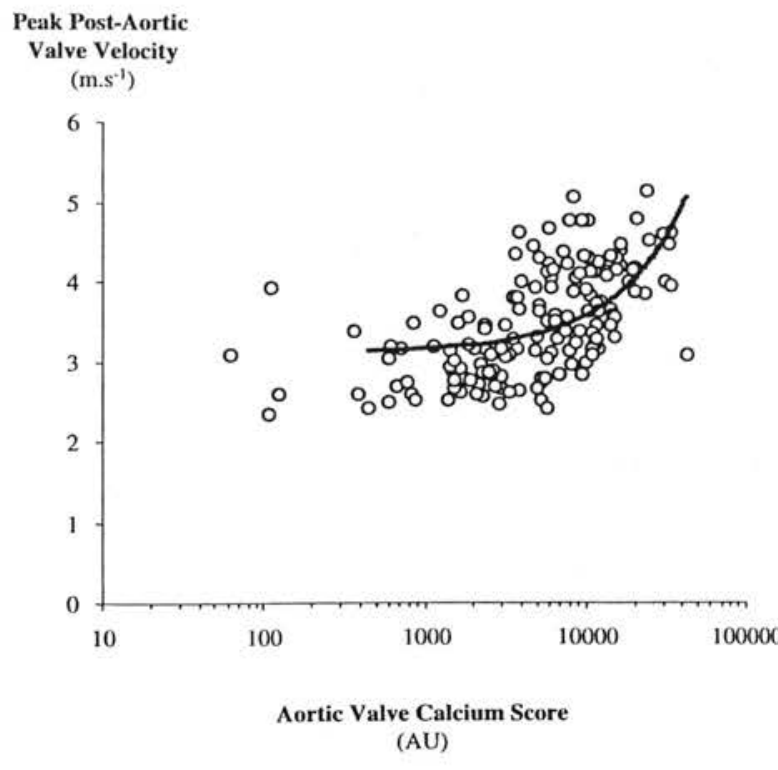

Fig. 2 - Correlation of aortic valve calcification and peak post-aortic valve velocity $(r=0.54, p<0.001)$. applicable to other computed tomography equipment. The reference ranges of the aortic valve calcium scores are likely to be dependent on the imaging protocol and CT equipment used. However, broadly speaking, poorly defined or diffuse segments of calcium usually represent a minor aortic valve gradient. In contrast, coalescent calcium centred on the aortic valve is likely to represent moderate stenosis whereas very heavy calcification almost invariably represents a significant degree of valvular stenosis (Fig. 1).

In conclusion, calcification of the aortic valve is closely associated with the severity of aortic stenosis and heavy

\section{Peak Post-Aortic \\ ValveVelocity}

$$
\left(\mathrm{m} . \mathrm{s}^{-1}\right)
$$
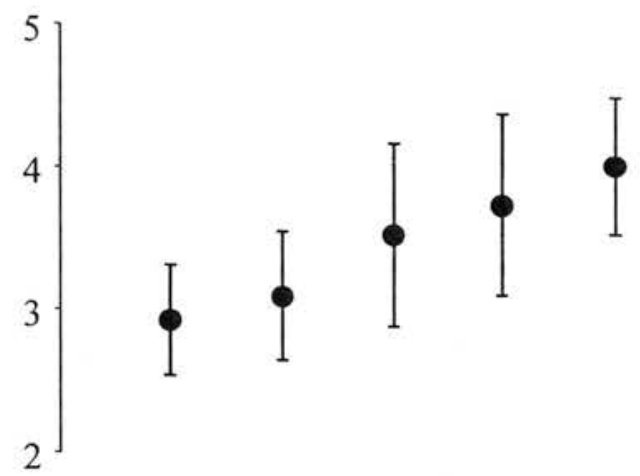

12345

Quintiles of Aortic Valve Calcium Score

Fig. 3 - Mean ( \pm SD) post-aortic valve velocity in the quintiles of aortic valve calcification. ANOVA $p<0.001$. Fisher's PLSD test $p \leq 0.03$ for all comparisons between the individual quintiles except quintile 1 vs quintile 2 . and quintile 3 versus quintile 4 . 
calcification suggests the presence of severe aortic stenosis that requires urgent cardiological assessment. Patients with lesser degrees of aortic valve calcification should be screened for aortic stenosis and monitored for disease progression.

Acknowledgements. This study was supported by a grant from the British Heart Foundation (PG/2000/044) and the additional support of the Wellcome Trust Clinical Research Facility in Edinburgh.

\section{REFERENCES}

1 Pomerance A. Pathogenesis of aortic stenosis and its relation to age. $\mathrm{Br}$ Heart J, 1972:34:569-574.

2 Otto CM, Kuusisto J, Reichenbach DD, et al. Characterization of the early lesion of "degenerative" valvular aortic stenosis: histologic and immunohistochemical studies. Circulation, 1994;90:844-853.

3 Wagner S. Selzer A. Patterns of progression of aortic stenosis: a longitudinal hemodynamic study. Circulation, 1982;65:709-712.

4 Rosenhek R, Binder T, Porenta G, et al. Predictors of outcome in severe, asymptomatic aortic stenosis. N Engl J Med, 2000;343:611-617.

5 Davies SW, Gershlick AH, Balcon R. Progression of valvar aortic stenosis: a long-term retrospective study. Eur Heart J, 1991;12:10-14.

6 Breen JF, Sheedy PF, Schwartz RS, et al. Coronary artery calcification detected with ultrafast $\mathrm{CT}$ as an indication of coronary artery disease. Radiology, 1992;185:435-439.

7 Mautner GC, Mautner SL. Froehlich J, et al. Coronary artery calcification: assessment with electron beam CT and histomorphometric correlation. Radiology, 1994;192:619-623.

8 Haberl R. Becker A, Leber A, et al. Correlation of coronary calcification and angiographically documented stenoses in patients with suspected coronary artery disease: results of 1764 patients. J Am Coll Cardiol, 2001;37:451-457.

9 Mautner GC, Roberts WC. Reported frequency of coronary arterial narrowing by angiogram in patients with valvular aortic stenosis. Am J Cardiol, 1992;70:539-540.

10 Woodring JH, West JW. CT of aortic and mitral valve calcification. $J K y$ Med Assoc, 1989;87:177-180.

11 Lippert JA, White CS, Mason AC, Plotnick GD. Calcification of aortic valve detected incidentally on CT scans: prevalence and clinical significance. AJR Am J Roentgenol, 1995;164:73-77.

12 Shemesh J, Apter S, Rozenman J, et al. Calcification of coronary arteries: detection and quantification with double-helix CT. Radiology, 1995; 197:779-783.

13 Bland JM, Altman DG. Statistical methods for assessing agreement between two methods of clinical measurement. Lancet, 1986; ; 307-310.

14 Kizer JR, Gefter WB, deLemos AS, et al. Electron beam computed tomography for the quantification of aortic valvular calcification. $J$ Heart Valve Dis, 2001;10:361-366.

15 Pohle K, Maffert R, Ropers D, et al. Progression of aortic valve calcification: association with coronary atherosclerosis and cardiovascular risk factors. Circulation, 2001;104:1927-1932.

16 Otto CM, Lind BK, Kitzman DW, et al. Association of aortic valve sclerosis with cardiovascular mortality and morbidity in the elderly. $N$ Engl J Med, 1999;341:142-147.

17 Wilmshurst PT, Stevenson RN, Griffiths H, Lord JR. A case control investigation of the relation between hyperlipidaemia and calcific aortic valve stenosis. Heart, 1997;78:475-479.

18 Chui MCK, Newby DE, Panarelli M, et al. Association between calcific aortic stenosis and hypercholesterolemia: is there a need for a randomised controlled trial of cholesterol lowering therapy? Clin Cardiol, 2001;24:52-55.

19 Callister TQ, Raggi P, Cooil B, et al. Effect of HMG-CoA reductase inhibitors on coronary artery disease as assessed by electron-beam computed tomography. N Engl J Med, 1998;339:1972-1978.

20 Shavelle DM, Takasu J, Budoff MJ, et al. HMG CoA reductase inhibitor (statin) and aortic valve calcium. Lancet, 2002;359:1125-1126.

21 Carr JJ, Crouse JR, Goff DC, et al. Evaluation of subsecond gated helical CT for quantification of coronary artery calcium and comparison with electron beam CT. AJR Am J Roentgenol, 2000;174:915-921. 


\title{
REVIEW
}

\section{Calcific aortic stenosis: same old story?}

\author{
S. Joanna Cowell', David E. Newby ${ }^{2}$, Nicholas A. Boon', Andrew T. Elder ${ }^{3}$ \\ 'Department of Cardiology, Royal Infirmary, 5 I Little France Crescent, Edinburgh EHI6 4SA, UK \\ ${ }^{2}$ Chancellor's Building, Royal Infirmary, 49 Little France Crescent, Edinburgh EHI6 4SB, UK \\ ${ }^{3}$ Department of Medicine for the Elderly, Western General Hospital, Crewe Road, Edinburgh EH4 2XU, UK
}

Address correspondence to: S. J. Cowell. Fax: (+44) 131242 6422. Email: jo.cowell@ed.ac.uk

\begin{abstract}
Calcific aortic stenosis is the commonest adult valvular heart condition seen in the western world. Its prevalence is continuing to rise, with predominance in older patients who are frequently undergoing successful aortic valve replacement. This review discusses the natural history of calcific aortic stenosis, highlights recent insights into its pathogenesis, and outlines current medical and surgical management. The potential role of novel therapeutic interventional strategies is discussed.
\end{abstract}

Keywords: aortic stenosis, aetiology, pathogenesis, management, medical therapy, elderly

\section{Introduction}

Aortic stenosis is the commonest adult heart valve condition seen in the western world. Over the last $30-50$ years, its diagnosis and management have been revolutionised by the development of invasive (cardiac catheterisation) and noninvasive (echocardiography) haemodynamic assessments as well as potentially curative cardiac surgery. Recent insights have been made into the pathogenesis of calcific aortic stenosis, resulting in speculation that the disease mimics atherosclerosis and progression could be delayed or prevented by the use of lipid lowering therapy. This exciting concept is currently under investigation in a number of centres and, if successful, may potentially reduce the need for aortic valve surgery.

\section{Epidemiology}

Calcific aortic stenosis was first documented in 1904 [1] and at that time was regarded as uncommon. In the 19 th century, calcific aortic stenosis was not recognised as a clinical entity since pathological studies revealed only cusp thickening and sclerosis [2]. As a result, aortic valve sclerosis (thickening without stenosis) and aortic valve stenosis were regarded as different pathological conditions for many decades. Recent evidence, however, suggests that they represent different stages of the same disease process [3-5]: sclerosis arising from the development of valvular calcific lesions that progress slowly over several decades before ultimately causing aortic stenosis [6]. The current prominence of calcific aortic valve disease is likely to represent increased human longevity associated with the declining prevalence of rheumatic fever.
Aortic valve sclerosis is present in $20-30 \%$ of individuals over 65 years and $48 \%$ over 85 years [7], and aortic stenosis in $2 \%$ and $4 \%$, respectively $[3,7,8]$. Calcific sclerosis and valvular stenosis occur in patients with both a normal tricuspid aortic valve as well as in those with a bicuspid valve. The prevalence of bicuspid aortic valves is difficult to determine but is estimated to affect $1-2 \%$ of the general population [9]. Up to $70 \%$ of patients with a bicuspid aortic valve develop valvular stenosis [9] and will require aortic valve replacement $1-2$ decades earlier in life (5th-6th decade) than in those with a tricuspid aortic valve.

\section{Natural history}

Prior to the introduction of haemodynamic assessment and cardiac surgery, the natural history of aortic stenosis was described by its clinical presentation. Calcific aortic stenosis is a gradually progressive disease, characterised by a long asymptomatic phase lasting several decades, followed by a shorter symptomatic phase usually associated with severe narrowing of the aortic valve orifice.

The outlook for patients with asymptomatic aortic stenosis is generally good and closely matches that of life table estimates for age- and sex-matched controls [10]. A striking feature of aortic stenosis is that the prognosis changes dramatically with the onset of symptoms in association with severe outflow obstruction: a 2 -year survival rate of $50 \%$. Although few studies specifically assessed the influence of age, patients over the age of 70 have a worse prognosis with 2 - and 3-year survival rates of $37 \%$ and $25 \%$, respectively [11]. The prognosis also depends upon the clinical presentation with a mean survival of 3 years for those presenting with angina 
and syncope, 2 years with the onset of breathlessness, and as little as 1 year in those who develop overt left ventricular failure $[12,13]$

\section{Other cardiovascular events}

Despite the favourable outlook in those patients with mild asymptomatic disease, there is an increased risk of cardiovascular events unrelated to the aortic valve disease. Otto and colleagues demonstrated that, in patients with aortic sclerosis, there is a $50 \%$ increased risk of myocardial infarction and cardiovascular death even in the absence of significant outflow tract obstruction [7]. The Helsinki Aging Study also suggested that patients with moderate to severe aortic stenosis had higher all cause and cardiovascular mortality irrespective of associated symptoms. In particular, a higher rate of stroke related death was noted although the majority of these patients had atrial fibrillation [14].

\section{Pathology of calcific aortic stenosis}

For many decades, calcific aortic stenosis has been attributed to prolonged 'wear and tear' and age-associated valvular degeneration. Contrary to this supposition, however, is the absence of aortic valve calcification or stenosis on echocardiography in a third of individuals over the age of 80 [8]. Recent evidence suggests that calcific aortic stenosis may result from an active inflammatory process involving biochemical, humoral and genetic factors.

\section{Histology}

Normal aortic valve leaflets are macroscopically smooth, thin and opalescent, with clearly defined tissue layers at a microscopic level and very few cells [15]. Increasing age gives rise to non-specific thickening of the tips of the valve leaflets, with an increase in the number of adipose cells and thinning of tissue layers [16]. In calcific aortic stenosis, there is characteristic leaflet thickening, with irregular nodular masses on the aortic aspect of the valve. Microscopic assessment of both mild and severely affected valves reveals endothelial and basement membrane disruption, with underlying subendothelial thickening. The lesion itself contains disorganised collagen fibres, chronic inflammatory cells, lipoproteins, lipid, extracellular bone matrix proteins and bone mineral $[15,16]$.

\section{Pathogenesis}

The histological features described closely resemble those seen in atherosclerosis and are strongly suggestive of chronic inflammation. In calcific aortic stenosis, the factors initiating the inflammatory process have not been identified but mechanical injury to the endothelium is thought to pave the way for subsequent inflammation. This concept is supported by the pattern of aortic valve cusp involvement that corresponds to areas of low shear and high tensile stress: namely the aortic surface of the leaflets and predilection for the non-coronary cusp $[17-20]$. Congenitally bicuspid aortic valves are less efficient than tricuspid valves at distributing mechanical stress and this may account for the more rapid development of stenosis [21].

\section{Role of lipids}

Endothelial injury or disruption may allow circulating lipids to enter the valvular interstitial tissue [22] and accumulate in areas of calcification and inflammation $[22,23]$. The lipoproteins implicated in atherogenesis, including low-density lipoprotein (LDL) and lipoprotein (a), are present in early aortic valve lesions [22] and undergo oxidative modification [23]. These oxidised lipoproteins are highly cytotoxic [24] and capable of stimulating inflammatory activity $[25,26]$ and mineralisation [27-29].

\section{Inflammation}

Both macrophages and activated $\mathrm{T}$ lymphocytes are present in the early and advanced lesions of congenitally bicuspid $[30]$ and tricuspid aortic valves [15, 16]. Migration of these effector inflammatory cells appears to be mediated through increased endothelial expression of cellular adhesion molecules such as E-selectin, intercellular adhesion molecule-1 (ICAM-1) and vascular cell adhesion molecule-1 (VCAM-1) $[31,32]$. Once recruited into the subendothelium, the inflammatory cells release enzymes, such as matrix metalloproteinases, that cause degradation of collagen, elastin and proteoglycans within the aortic valve cusps [33].

\section{Calcification}

Mineralisation is a characteristic of both atherosclerotic and aortic valve lesions, and arises in close proximity to areas of inflammation. It is a prominent feature in calcific aortic stenosis and has been demonstrated in early [16] as well as advanced lesions [34]. Surgically excised valves have even revealed areas of mature lamellar bone, haemopoietic marrow and bone remodelling [34]. Several features suggest the presence of an active highly regulated process closely resembling developmental bone formation $[35,36]$.

The initiation of mineralisation (nucleation) may be stimulated by the presence of cellular degradation products following apoptosis [37] or by the presence of oxidised lipids $[23,34]$. In vitro studies of cultured explants of stenotic valves have identified cells with osteoblastic characteristics capable of phenotypic differentiation and spontaneous calcification [38]. Their origin is unknown but they may be derived from a pool of circulating immature pluripotent mesenchymal cells [39]. These osteogenic cells or 'calcifying valvular cells' express and produce a variety of regulatory bone matrix proteins including osteopontin [40, 41] and bone morphogenetic protein [34].

\section{Similarities and differences with atherosclerosis}

Although the similarities with atherosclerosis were recognised as long ago as 1917 [42], they were largely disregarded until recently [43-45]. The histological studies described above have highlighted the common features but also confirmed differences in the cellular and mineral components of the two lesions.

Smooth muscle proliferation and lipid-laden macrophages (or foam cells) are prominent features of vascular atheroma but are virtually absent in stenotic aortic valves. In

\section{Page 2 of 7}


addition, mineralisation is an earlier and more extensive feature of aortic valve lesions compared with atherosclerosis [16. These differences may, in part, explain why only $40 \%$ of patients with severe aortic stenosis have significant coronary artery disease $[46-50]$ and why the majority of patients with coronary artery disease do not have aortic stenosis. As the underlying pathology for the two conditions appears to be similar, it is likely that other unknown factors influence the development of valvular as opposed to vascular lesions [51].

\section{Clinical presentation}

Patients present with either an incidentally noted asymptomatic systolic murmur or with symptoms of severe disease including angina, exertional syncope, breathlessness, and reduced exercise tolerance or lethargy. In simple terms, progressive obstruction to outflow results in a gradual rise in left ventricular pressures, left ventricular hypertrophy, and diastolic dysfunction. Once the degree of stenosis is severe, further small decreases in aortic valve area result in large changes in the pressure gradient across the valve. Symptoms and decompensation arise due to the development of inadequate cardiac reserve, myocardial oxygen demand mismatch or pressure overload of the left ventricle. Symptoms rarely occur unless the degree of stenosis is of at least moderate severity (with an aortic valve area of less than $1.0 \mathrm{~cm}^{2}$ ) but patients may remain asymptomatic for long periods with even very severe stenosis [46].

\section{Clinical risk factors}

In keeping with the apparent parallels with atherosclerosis, calcific aortic stenosis is associated with coronary artery disease $[48,50]$ and many of its risk factors (Table 1) [3]. Calcific aortic stenosis is also seen in association with severe homozygous familial hypercholesterolaemia, and its development appears to be influenced by the length of exposure to elevated serum cholesterol concentrations [52]. Interest-

Table I. Risk factors for calcific aortic stenosis

\begin{tabular}{ll}
\hline Clinical & Biochemical \\
Age & Hyperlipidacmia (LDL and LP (a)) \\
Male sex & $\begin{array}{l}\text { Hypercalcaemia } \\
\text { Smoking }\end{array}$ \\
$\begin{array}{l}\text { Hypertension } \\
\text { Diabetes mellitus } \\
\text { Coronary artery discase } \\
\text { Chronic renal failure } \\
\text { Paget's disease }\end{array}$ \\
Hyperparathyroidism
\end{tabular}

LDL = Low-density lipoprotein; LP (a) = Lipoprotein a. ingly, aggressive lipid lowering therapy with plasmapheresis has been reported to regress aortic stenosis in such patients [53]. Milder forms of hypercholesterolaemia have also been associated with calcific aortic stenosis $[54,55,56]$, particularly in patients with non-rheumatic tricuspid valves [54].

Conditions affecting calcium metabolism, such as chronic renal impairment with secondary hyperparathyroidsim [5759] and advanced Paget's disease [60], predispose individuals to aortic valve calcification and accelerated stenosis. Such patients also tend to have diffuse cardiac calcification affecting the mitral valve, myocardium and conducting system.

A number of twin studies and case reports suggest that hereditary factors may influence the development of calcific aortic valve stenosis $[61,62]$. There has been a single report of a genetic association between aortic stenosis and a vitamin D receptor polymorphism [63] but this finding has yet to be confirmed.

\section{Investigations}

The assessment of valvular stenosis and monitoring of disease progression has only been possible over the last five decades using cardiac catheterisation, echocardiography and more recently magnetic resonance (MR) imaging and computed tomography (CT). Magnetic resonance may have some advantages over echocardiography in assessment of stenosis severity [64], but its availability is limited and measurements are time consuming to perform. Although currently limited to clinical research, CT has recently been validated as an accurate means of quantifying aortic valve calcification, a measure that correlates well with the severity of stenosis estimated by echocardiography [65]. Echocardiography remains the current gold standard for monitoring of disease progression and left ventricular function in patients with aortic stenosis.

The severity of aortic valve stenosis is assessed using both two-dimensional and Doppler echocardiography (Table 2). Narrowing of the aortic valve orifice results in acceleration of blood flow across the valve. Using spectral Doppler, the velocity of blood passing through the left ventricular outflow tract (pre-valve) and aortic valve orifice (post-valve) can be measured and is usually expressed in metres per second. The peak instantaneous pressure gradient across the aortic valve has a simple relationship with the peak post-valve velocity and is described as four times the square of the velocity (modified Bernoulli equation). For example, a peak post-valve velocity of $4 \mathrm{~m} / \mathrm{s}$ gives an instantaneous pressure gradient of $4 \times 4^{2}=64 \mathrm{mmHg}$. Where there are concerns that impaired left ventricular function limits the ability to generate an adequate pressure gradient across the valve, measurement of the aortic valve area may

Table 2. Echocardiographic measures of severity of aortic stenosis ( $A S)$

\begin{tabular}{lllll}
\hline & Normal & Mild AS & Moderate AS & Severe AS \\
Pcak post-valve velocity $(\mathrm{m} / \mathrm{s})$ & $0.9-1.8$ & $2.5-3.0$ & $3.0-4.0$ & $>4.0$ \\
Peak gradient $(\mathrm{mmHg})$ & $<25$ & $25-36$ & $36-64$ & $>64$ \\
Aortic valve area $\left(\mathrm{cm}^{2}\right)$ & $2.0-3.5$ & $1.0-2.0$ & $0.5-1.0$ & $<0.5$ \\
\hline
\end{tabular}




\section{S. J. Cowell et al.}

need to be made using direct planimetry or indirectly using the continuity equation. On occasions, dobutamine stress echocardiography may be used as method of distinguishing true aortic stenosis causing left ventricular dysfunction from aortic pseudostenosis where the impairment of the left ventricle causes poor excursion of the aortic valve cusps giving the impression of stenotic valvular restriction.

\section{Disease progression}

Echocardiography provides the most accurate evaluation of disease progression, which can be unpredictable and extremely variable. Some individuals show little or no evidence of deterioration over time, yet others progress rapidly from mild to severe stenosis within a few years.

In patients with aortic valve sclerosis, progression to stenosis (arbitrarily defined as a peak post-valve velocity $\geq 2.5 \mathrm{~m} / \mathrm{s}$, or peak gradient $\geq 25 \mathrm{mmHg}$ ) is a relatively slow process with mean increases in peak post valve velocity and peak gradient of $0.07 \mathrm{~m} / \mathrm{s}$ and $1.4 \mathrm{mmHg}$ per year, respectively [66] However, once the valve is classified as stenotic, disease progression is more rapid with average increases of $0.3 \mathrm{~m} / \mathrm{s}$ and $7-8 \mathrm{mmHg}$ per year, corresponding to a decrease in aortic valve area of $0.1 \mathrm{~cm}^{2}$ per year [67-71].

\section{Predictors of progression and clinical outcome}

Disease progression and clinical outcome have been linked to many of the risk factors for calcific aortic stenosis, including age, male sex, hyperlipidaemia, hypertension, diabetes mellitus, smoking, hypercalcaemia and chronic renal impairment $[69,72,73-75]$. However, much of the evidence is conflicting and limited by the retrospective nature of the studies. The most consistent and strongest predictors of disease progression are severity of stenosis at baseline [71] and degree of valvular calcification $[72,76,77]$. The more severe the stenosis at baseline and the more heavily calcified the valve, the faster the rate of progression. Clinical outcome is also influenced by the degree of valvular calcification, with nearly $80 \%$ of patients with moderate to severe calcification who progress rapidly $(>0.3 \mathrm{~m} / \mathrm{s} / \mathrm{yr})$ either dying or undergoing aortic valve replacement within 2 years [77].

\section{Management of calcific aortic stenosis}

$\Lambda t$ the present time, there is no known therapy that can slow or reverse disease progression in patients with calcific aortic stenosis. Current management includes monitoring disease progression, and ensuring patient awareness of the need for antibiotic prophylaxis against infective endocarditis. For those patients with severe symptomatic disease, the therapeutic options include conventional medical therapy for symptom control and aortic valve replacement.

\section{General advice}

All patients should be advised of the need for antibiotic prophylaxis against endocarditis for dental and other invasive procedures. Patients with moderate or severe disease should be advised to avoid strenuous physical exercise and competitive sport, and to report promptly the onset of symptoms.

\section{Monitoring of disease progression}

Since disease progression is so unpredictable, the majority of patients should be reviewed regularly to monitor changes in stenosis severity and watch for the onset of symptoms. As a rule of thumb, asymptomatic patients with mild to moderate stenosis require review and echocardiography every 1-2 years, and those with moderate to severe stenosis every 612 months. Patients developing symptoms between appointments should be reviewed immediately.

\section{Asymptomatic severe aortic stenosis}

One contentious area of management is determining the optimal timing for aortic valve replacement. It is universally accepted that surgery is indicated as soon as symptoms appear in patients with severe stenosis. Although many cardiologists are loath to refer patients without symptoms for valve surgery, there are some who feel uncomfortable managing patients with severe asymptomatic valvular stenosis because of the potential risk for sudden cardiac death. However, this is rare and occurs in less than $1 \%$ of asymptomatic patients per year [78]. The combined risk of aortic valve replacement $(2-10 \%$ mortality) and prosthesis-related complications $(2-3 \%$ /year) is thus greater than the risk of sudden cardiac death. 'Watchful waiting' is therefore recommended.

The onset of symptoms in patients with severe stenosis may be subtle and insidious, particularly in the elderly where co-morbidity may mislead or obscure the presentation. For this reason careful history taking for changes in exercise tolerance as well as the classical symptoms of breathlessness, chest pain and syncope is required. In cases where patients may be underplaying symptoms, attributing them to 'old age', or unknowingly avoiding activity that induces symptoms, physician supervised exercise testing may be helpful in both revealing symptoms as well as determining the haemodynamic response to exercise. Patients who develop symptoms during exercise, become hypotensive, manifest marked ST segment changes or develop ventricular arrhythmias are at high risk and should be referred for valve replacement [79-81].

\section{Symptomatic severe aortic stenosis}

As soon as patients with severe aortic stenosis develop symptoms the treatment of choice is aortic valve replacement because this substantially improves quality of life and prognosis. In those patients declining valve surgery, or the frail elderly in whom major cardiac surgery would be inappropriate, palliation with conventional medical therapy, or in exceptional circumstances, balloon valvotomy are the only alternatives. Percutaneous aortic valve replacement is a promising new technique that is currently under development in highly selected patient populations $[82,83]$.

\section{Medical therapy}

\section{Breathlessness}

Patients with evidence of pulmonary congestion may benefit from the judicious use of diuretics, vasodilators and positive inotropic agents such as digoxin. Excessive use of diuretics should be avoided since patients with severe aortic 


\section{Calcific aortic stenosis}

stenosis often have diastolic dysfunction and depend on an adequate pre-load in order to maintain their cardiac output.

Despite the widespread belief that ACE inhibitors can cause dangerous hypotension in severe aortic stenosis, and are therefore contraindicated, there are little data to support this. From the limited literature available, two small studies demonstrated that first dose hypotension did not occur in patients with severe aortic stenosis, and that cardiac output and symptoms improved substantially $[84,85]$. Although further study is required, some patients with heart failure and severe aortic stenosis could benefit from ACE inhibitors provided that they are carefully introduced in a hospital setting. Certainly those patients already established on therapy need not have it withdrawn since this may precipitate the onset of heart failure.

Digoxin can be helpful in the management of heart failure but should only be used in the presence of atrial fibrillation or where there is documented evidence of left ventricular systo lic dysfunction. Atrial fibrillation is not well tolerated in the presence of severe stenosis and restoration to sinus rhythm (through DC cardioversion or pharmacological cardioversion using amiodarone) should be attempted wherever possible.

\section{Angina}

In those individuals where angina is the predominant symptom, cautious use of beta blockers and nitrates may be of benefit.

\section{Syncope}

Patients with syncope or pre-syncope should be further evaluated with a 24 -hour cardiac monitor since aortic stenosis is commonly associated with atrioventricular block. There is no specific therapy for syncope unless it is caused by a bradyarrhythmia or tachyarrhythmia, where pacemaker insertion or antiarrhythmic therapy, respectively, should be considered.

\section{Balloon valvotomy}

Although balloon valvotomy plays an important role in the management of adolescents and young adults with aortic stenosis, it has largely been abandoned in older patients. The functional improvement obtained is limited, the restenosis and complication rates are high, and the long term outlook poor ( $<80 \%$ survival at 1 year) $[78,86]$. On rare occasions, balloon valvotomy may play a role in patients with a limited life expectancy for other reasons, or as a bilige to aurtic valve replacement in critically ill patients with cardiogenic shock.

\section{Aortic valve replacement}

Aortic valve replacement incurs the virtual abolition of symptoms associated with improvements in physical functioning and quality of life, and a dramatic improvement in survival. Operative mortality in middle-aged adults is in the region of $5-8 \%[87,88,89]$ with 5 - and 10 -year survival rates of approximately $80 \%[87,90]$ and $65 \%$, respectively [87], which approaches actuarial survival rates for the general population [87].
Factors associated with a higher operative mortality include increasing age [91], the presence of renal impairment, cerebrovascular and peripheral vascular disease [92], the presence of impaired left ventricular function [91], and the need for simultaneous coronary artery bypass grafting [88]. Despite the increased operative risk associated with the presence of left ventricular failure, this is not an absolute contra-indication to surgery. Indeed these patients may have the most to gain from valve surgery in terms of improvements in prognosis.

\section{Aortic valve replacement in octogenarians}

Successful aortic valve replacement is becoming increasingly common in patients over the age of 80 . Despite evidence suggesting that it should be offered to all suitable patients regardless of age, several studies have demonstrated a reluctance to refer older patients for valve surgery $[8,93,94]$. This probably reflects both patient and physician miscon ceptions of the risks and benefits of operative intervention.

Although operative mortality is higher in octogenarians (nearer $5-15 \%$ ), these individuals have almost as much to gain as their younger counterparts in terms of improved prognosis (5-year survival being 55-70\%). Of perhaps greater importance is that the majority of survivors achieve a significant reduction in symptoms $[92,95,96,97,98]$ associated with a marked improvement in physical functioning and quality of life $[88,95,96,98]$. Although intensive care $[92,98]$ and overall hospital stay $[95,97,98]$ may be longer, the majority return to their own homes and retain their independence on discharge $[92,95]$. However, post-operative complications are more common with a higher incidence particularly of stroke (4\%) and acute renal failure (7-10\%) [98]. In contrast to younger patients, octogenarians are usually offered a bioprosthetic (as opposed to a mechanical) valve, thus reducing the risk of valve thrombosis and anticoagulant associated haemorrhage.

\section{Potential role for HMG CoA reductase inhibitors}

Hydroxymethylglutaryl coenzyme A (HMG CoA) reductase inhibitors or statins are now well established in the primary and secondary prevention of coronary artery dis ease $[99,100]$. Several studies have also shown that these drugs can cause regression of coronary artery disease [101] as well as reduce the calcific volume of coronary plaques, [102]. Given the clinical association of calcific aortic stenosis with hyperlipidaemia and coronary artery disease, and the striking histological similarities with atheroma, the speculation that statins may have the potential to influence disease progression in aortic stenosis is an intriguing hypothesis $[103,104]$.

Recent retrospective studies [105-109] have demonstrated that statins may delay disease progression in aortic stenosis through their lipid-lowering and anti-inflammatory actions [109]. These observational data should be interpreted with caution since none of these studies was randomised, and the statin doses were small. This preliminary evidence has been the rationale for establishing several ongoing randomised controlled trials of statin therapy in patients with aortic stenosis, such as the Scottish Aortic stenosis and 


\section{S. J. Cowell et al.}

Lipid lowering Therapy, Impact on REgression (SALTIRE) and Simvastatin and Ezetimibe in Aortic Stenosis (SEAS) trials.

\section{Conclusion}

The need for an alternative to aortic valve surgery is highlighted by the increasing longevity of the population and rising prevalence of aortic stenosis. New therapeutic strategies to limit disease progression are needed in order to delay, and potentially avoid, the need for valve surgery. The outcomes of several ongoing randomised controlled trials investigating the role of lipid-lowering therapy in aortic stenosis are awaited with interest.

\section{Key points}

- Aortic stenosis is increasingly common.

- Severe aortic stenosis in the presence of symptoms carries a very poor prognosis.

- Aortic valve replacement dramatically improves survival and quality of life, even in octogenarians.

- Too few older patients are offered aortic valve replacement.

- Lipid-lowering therapy may have a potential role in the prevention of disease progression.

\section{Conflicts of interest declaration}

The authors are currently involved in the SALTIRE trial funded by the British Heart Foundation with an additional educational grant award from Pfizer (JK) Limited, as well as the SEAS study which is sponsored by Merck Sharp and Dohme Limited.

\section{Please note}

The very long list of references supporting this review has meant that only the most important are listed here and are represented by bold type throughout the text. The full list of references is available on the journal website (http://www. ageing.oupjournals.org).

\section{References}

3. Stewart BF, Siscovick D, Lind BK $c$ al. for the Cardiovascular Health Study. Clinical factors associated with calcific aortic valve disease. J Am Coll Cardiol 1997; 29: 630-4.

7. Otto CM, Lind BK, Kitzman DW, Gersch BJ, Siscovick DS. Association of aortic-valve sclerosis with cardiovascular mortality and morbidity in the elderly. N Engl J Med 1999; 341: $142-7$.

8. Lindroos M, Kupari M, Heikkila J, Tilvis R. Prevalence of aortic valve abnormalities in the elderly: an echocardiographic study of a random population sample. J Am Coll Cardiol 1993; 21: $1220-5$.

14. livanainen AM, Lindroos M, Tilvis R, Heikkila, Kupari M. Natural history of aortic valve stenosis of varying severity in the elderly. Am J Cardiol 1996; 78: 97-101.
16. Otto CM, Kuusisto J, Reichenbach DD, Gown AM, O'Brien $\mathrm{KD}$. Characterization of the early lesion of 'degenerative' valvular aortic stenosis: histologic and immunohistochemical studies. Circulation 1994; 90: 844-53.

22. O’Brien KD, Reichenbach DD, Marcovina SM, Kuusisto J, Alpers CE, Otto CM. Apolipoprotein B, (a), and E accumulate in the morphologically early lesion of 'degenerative' valvular aortic stenosis. Arterioscler Thromb Vasc Biol 1996; 16: 523-32.

23. Olsson M, Thyberg J, Nilsson J. Presence of oxidised low density lipoprotein in nonrheumatic stenotic aortic valves. Arterioscler Thromb Vasc Biol 1999; 19: 1218-22.

29. Parhami F, Morrow AD, Balucan J et al. Lipid oxidation products have opposite effects on calcifying vascular cell and bone cell differentiation. A possible explanation for the paradox of arterial calcification in osteoporotic patients. Arterioscler Thromb Vasc Biol 1997; 17: 680-7.

34. Mohler ER 3rd, Gannon F, Reynolds C, Zimmerman R, Keane MG, Kaplan FS. Bone formation and inflammation in cardiac valves. Circulation 2001; 103: 1522-8.

36. Demer LL. A skeleton in the atherosclerosis closet. Circulation 1995; 92: 2029-32.

45. Demer LL. Cholesterol in vascular and valvular calcification. Circulation 2001; 104: 1881-3.

50. Peltier M, Trojette F, Sarano ME, Grigioni F, Slama MA, Tribouilloy CM. Relation between cardiovascular risk factors and nonrheumatic severe calcific aortic stenosis among patients with a three-cuspid aortic valve. $\mathrm{Am}$ J Cardiol 2003; 91: 97-9.

51. Otto CM, O' Brien KD. Why is there discordance between calcific aortic stenosis and coronary artery disease? Heart 2001; 85: 601-2.

54. Chui MC, Newby DE, Panarelli M, Bloomfield P, Boon NA. Calcific aortic stenosis and hypercholesterolaemia: a causal association? Heart 1999; 81: 171.

65. Cowell SJ, Newby DE, Burton J et al. Aortic valve calcification on computed tomography predicts the severity of aortic stenosis. Clin Radiol 2003; 58: 712-6.

66. Faggiano P, Antonini-Canterin F, Erlicher A et al. Progression of aortic valve sclerosis to aortic stenosis. Am J Cardiol 2003; 91: 99-101.

71. Otto CM, Burwash IG, Legget ME et al. Prospective study of asymptomatic valvular aortic stenosis. Clinical, echocardiographic, and exercise predictors of outcome. Circulation 1997; 95: 2262-70.

73. Palta S, Pai AM, Gill KS, Pai RG. New insights into the progression of aortic stenosis. Implications for secondary prevention. Circulation 2000; 101: 2497-502.

77. Rosenhek R, Binder T, Porenta G et al. Predictors of outcome in severe, asymptomatic aortic stenosis. N Engl J Med 2000; 343: 611-17.

78. Bonow RO, Carabello B, de Leon AC et al. ACC/AHA guide lines for the management of patients with valvular heart disease. J Am Coll Cardiol 1998; 32: 1486-588.

81. Carabello BA. Evaluation and management of patients with aortic stenosis. Circulation 2002; 105: 1746-50.

87. Linblom D, Lindblom U, Qvist J, Lundström. Long-term survival rates after heart valve replacement. J Am Coll Cardiol 1990; 15: 566-73.

88. Sprigings DC, Forfar JC. How should we manage symptomatic aortic stenosis in the patient who is 80 or older? $\mathrm{Br}$ Heart J 1995; 74: 481-4.

93. Bouma BJ, van den Brink RBA, van der Meulen JHP et al. To operate or not on elderly patients with aortic stenosis: the decision and its consequences. Heart 1999; 82: 143-8.

\section{Page 6 of 7}




\section{Calcific aortic stenosis}

94. Abdul-Hamid AR, Mulley GP. Why do so few older people with aortic stenosis have valve replacement surgery? Age Ageing 1999; 28: 261-4.

97. Kohl P, Kerzmann A, Lahaye L, Gerard P, Limet R. Cardiac surgery in octogenarians. Peri-operative outcome and longterm results. Eur Heart J 2001; 22: 1235-43.

98. Sundt TM, Bailey MS, Moon MR et al. Quality of life after aortic valve replacement at the age of $>80$ years. Circulation 2000; 102 (Suppl III): III70-74.

103. Pearlman AS. Medical treatment of aortic stenosis. Promising, or wishful thinking? J Am Coll Cardiol 2002; 40: 1731-4.
104. Mohler ER. Are atherosclerotic processes involved in aortic valve calcification? Lancet 2000; 356: 524-5.

109. Bellamy MF, Pellikka PA, Klarich KW, TajikAJ, EnriquezSarano M. Association of cholesterol levels, Hydroxymethylglutaryl coenzyme- $A$ reductase inhibitor treatment, a progression of aortic stenosis in the community. J Am Coll Cardiol 2002; 40: $1723-30$.

Received 17 September 2003; accepted 8 April 2004 


\title{
A Randomized Trial of Intensive Lipid-Lowering Therapy in Calcific Aortic Stenosis
}

\author{
S. Joanna Cowell, B.M., David E. Newby, M.D., Robin J. Prescott, Ph.D., \\ Peter Bloomfield, M.D., John Reid, M.B., Ch.B., David B. Northridge, M.D., \\ and Nicholas A. Boon, M.D., for the Scottish Aortic Stenosis \\ and Lipid Lowering Trial, Impact on Regression (SALTIRE) Investigators
}

ABSTRACT

\section{BACKGROUND}

Calcific aortic stenosis has many characteristics in common with atherosclerosis, including hypercholesterolemia. We hypothesized that intensive lipid-lowering therapy would halt the progression of calcific aortic stenosis or induce its regression.

\section{METHODS}

In this double-blind, placebo-controlled trial, patients with calcific aortic stenosis were randomly assigned to receive either $80 \mathrm{mg}$ of atorvastatin daily or a matched placebo. Aortic-valve stenosis and calcification were assessed with the use of Doppler echocardiography and helical computed tomography, respectively. The primary end points were change in aortic-jet velocity and aortic-valve calcium score.

\section{RESULTS}

Seventy-seven patients were assigned to atorvastatin and 78 to placebo, with a median follow-up of 25 months (range, 7 to 36 ). Serum low-density lipoprotein cholesterol concentrations remained at $130 \pm 30 \mathrm{mg}$ per deciliter in the placebo group and fell to $63 \pm 23 \mathrm{mg}$ per deciliter in the atorvastatin group $(\mathrm{P}<0.001)$. Increases in aortic-jet velocity were $0.199 \pm 0.210 \mathrm{~m}$ per second per year in the atorvastatin group and $0.203 \pm 0.208 \mathrm{~m}$ per second per year in the placebo group $(\mathrm{P}=0.95$; adjusted mean difference, $0.002 ; 95$ percent confidence interval, -0.066 to $0.070 \mathrm{~m}$ per second per year). Progression in valvular calcification was $22.3 \pm 21.0$ percent per year in the atorvastatin group, and $21.7 \pm 19.8$ percent per year in the placebo group $(\mathrm{P}=0.93$; ratio of posttreatment aortic-valve calcium score, $0.998 ; 95$ percent confidence interval, 0.947 to 1.050).

\section{CONCLUSIONS}

Intensive lipid-lowering therapy does not halt the progression of calcific aortic stenosis or induce its regression. This study cannot exclude a small reduction in the rate of disease progression or a significant reduction in major clinical end points. Long-term, large-scale, randomized, controlled trials are needed to establish the role of statin therapy in patients with calcific aortic stenosis.

From the Department of Cardiology, Royal Infirmary, Edinburgh (S.J.C., D.E.N., P.B., N.A.B.); Public Health Sciences, University of Edinburgh Medical School, Edinburgh (R.J.P.); the Department of Radiol. ogy, Borders General Hospital, Melrose, Roxburghshire, United Kingdom (J.R.); and the Department of Cardiology, Western General Hospital, Edinburgh (D.B.N.). Address reprint requests to $\mathrm{Dr}$. Newby at the Department of Cardiology, Royal Infirmary, Old Dalkeith Rd., Little France, Edinburgh EHI6 4SU, United Kingdom, or at d.e.newby@ed.ac.uk.

N EnglJ Med 2005;352:2389-97. Copyright (1) 2005 Massachusetts Medical Society 
N THE WESTERN WORLD, CALCIFIC AORTIC stenosis is the most common form of valvular heart disease, and its incidence increases with age such that 3 percent of adults over 75 years of age have aortic stenosis. ${ }^{1}$ It is a gradually progressive disease, characterized by a long asymptomatic phase, lasting several decades, followed by a shorter symptomatic phase associated with severe narrowing of the orifice of the aortic valve. Once symptoms occur, the prognosis is poor and surgery is usually mandated. Calcific aortic stenosis is now the leading indication for valve replacement in North America and Europe. However, there are currently no effective disease-modifying treatments, and the possibility of halting the disease process would represent a therapeutic advance.

Calcific aortic stenosis is mediated by a chronic inflammatory disease process that has many similarities with atherosclerosis and includes inflammatory-cell infiltrates, lipoproteins, lipids, extracellular-bone-matrix proteins, and bone mineral. ${ }^{2-5}$ Consistent with these observations, clinical studies have revealed a strong association with coronary artery disease ${ }^{6,7}$ and many of its risk factors, including hypercholesterolemia. ${ }^{1}$ Disease progression in aortic stenosis is variable and influenced by several factors, including the degree of stenosis, ${ }^{8}$ valvular calcification, ${ }^{9-11}$ and hypercholesterolemia. ${ }^{12,13}$ Indeed, calcific aortic stenosis is a feature of severe homozygous familial hypercholesterolemia, ${ }^{14}$ and intensive lipid-lowering therapy with plasmapheresis can reverse valvular stenosis in patients with this disease. ${ }^{15}$

The use of hydroxymethylglutaryl-coenzyme A reductase inhibitors, or statins, is an established treatment for the primary and secondary prevention of coronary artery disease. ${ }^{16,17}$ Several studies have shown that these drugs can halt the progression of coronary artery disease $\mathrm{e}^{18-20}$ and reduce coronary calcification. ${ }^{21-23}$ Given the clinical association with hypercholesterolemia and coronary artery disease, and the histologic similarities with atheroma, it has been suggested that statin therapy may halt the progression, or even induce regression, of calcific aortic stenosis. This hypothesis is supported by numerous retrospective observational studies ${ }^{24-29}$ showing that concomitant statin therapy was associated with a delay in disease progression, demonstrated by a reduction of $0.30 \mathrm{~m}$ per second peryear in the rate of change in aortic-jet velocity, and of 30 percent per year in valvular calcification.

The aim of the Scottish Aortic Stenosis and Lipid
Lowering Trial, Impact on Regression (SALTIRE) was to establish whether intensive lipid-lowering therapy with $80 \mathrm{mg}$ of atorvastatin daily would halt the progression or induce regression of the aorticjet velocity on Doppler echocardiography, and of the aortic-valve calcium score on computed tomography $(\mathrm{CT})$, in patients with calcific aortic stenosis.

\section{METHODS}

\section{PATIENTS}

Patients older than 18 years of age with calcific aortic stenosis, an aortic-jet velocity of at least $2.5 \mathrm{~m}$ per second, and aortic-valve calcification on echocardiography ${ }^{11}$ were eligible for inclusion. Exclusion criteria were child-bearing potential without contraception, active or chronic liver disease, a history of alcohol or drug abuse, severe mitral-valve stenosis (mitral-valve area, $<1 \mathrm{~cm}^{2}$ ), severe mitral or aortic regurgitation, ${ }^{30}$ left ventricular dysfunction (ejection fraction, $<35$ percent), a planned aortic-valve replacement, intolerance of statins, statin therapy or a potential benefit from statin therapy (according to the treating physician), a baseline serum total cholesterol concentration of less than $150 \mathrm{mg}$ per deciliter ( $4.0 \mathrm{mmol}$ per liter), and presence of a permanent pacemaker or cardiodefibrillator. Of the patients screened, 455 were eligible for inclusion, 173 agreed to participate, and 155 ultimately underwent randomization.

\section{STUDY PROTOCOL}

Between March 2001 and April 2002, the blinded study coordinator randomly assigned eligible patients by the minimization technique ${ }^{31}$ with the use of a dedicated, locked computer program (Edinburgh University) incorporating the following eight variables: age, sex, smoking habit, hypertension, diabetes mellitus, serum cholesterol concentration, aortic-jet velocity, and aortic-valve calcium score. Patients were assigned to either $80 \mathrm{mg}$ of atorvastatin (Lipitor, Pfizer) or matched placebo as a single daily dose. Numbered containers were used.

Patients were assessed at baseline, two months, and six months and every six months thereafter for a minimum of two years. Clinical evaluation included assessment of functional status and adverse events, and the biochemical analysis of blood. Echocardiography and CT were performed at baseline, at each annual visit, and before withdrawal from the study. Patients who underwent randomization and who were subsequently started on open- 
label statin therapy by their attending physician were immediately scanned and withdrawn from the study.

Drs. Cowell, Reid, Northridge, and Bloomfield collected the data; Drs. Newby, Northridge, and Boon designed the study and vouch for the data and the analysis; Dr. Prescott analyzed the data; and all investigators participated in writing the article. The drug and the placebo were provided by Pfizer, who had no other input into the study. The investigation conformed to the Declaration of Helsink and was approved by all regional ethics committees. All patients gave written informed consent.

\section{ECHOCARDIOGRAPHY}

Assessment of valvular stenosis was determined by a single dedicated research ultrasonographer. $\mathrm{Pa}$ tients were studied with the use of a $3-\mathrm{MHz}$ transducer for $\mathrm{M}$-mode (single-dimensional) and pulsed and continuous-wave Doppler scanning. All measurements were determined online, averaged from three cardiac cycles (five cycles if the patient was in atrial fibrillation), and recorded onto super-VHS videotape and optical disk according to a standard protocol.

Peak and mean aortic-valve pressure gradients were calculated with the Bernoulli equation, and aortic-valve area was calculated with the continuity equation. The severity of aortic stenosis was determined with echocardiography according to the following standard guidelines: normal is defined by a peak velocity of 1.0 to $2.0 \mathrm{~m}$ per second, peak and mean gradients of $0 \mathrm{~mm} \mathrm{Hg}$, and a valve area of greater than $2.0 \mathrm{~cm}^{2}$; mild by a peak velocity of 2.1 to $3.0 \mathrm{~m}$ per second, a peak gradient of 16 to $35 \mathrm{~mm} \mathrm{Hg}$, a mean gradient of less than $15 \mathrm{~mm} \mathrm{Hg}$, and a valve area of 2.0 to $1.3 \mathrm{~cm}^{2}$; moderate by a peak velocity of 3.1 to $4.0 \mathrm{~m}$ per second, a peak gradient of 36 to $64 \mathrm{~mm} \mathrm{Hg}$, a mean gradient of 15 to $50 \mathrm{~mm} \mathrm{Hg}$, and a valve area of 1.2 to $0.8 \mathrm{~cm}^{2}$; and severe by a peak velocity of greater than $4.0 \mathrm{~m}$ per second, a peak gradient of greater than $64 \mathrm{~mm} \mathrm{Hg}$, a mean gradient of greater than $50 \mathrm{~mm} \mathrm{Hg}$, and a valve area of less than $0.8 \mathrm{~cm}^{2}$.

\section{COMPUTED TOMOGRAPHY}

CT was performed by a single operator with the use of a double-helix scanner (Twin II Flash, Philips Medical Systems) calibrated against a standard phantom. The region of the aortic valve was scanned with a spiral CT with the use of $2.7-\mathrm{mm}$ slices, a pitch of 0.7 , and an increment of $1.4 \mathrm{~mm}$ during inspiratory breath-holding sessions. All images were analyzed by a single operator with the use of automated computerized software (Picker Cardiac Scoring), involving a modified Agatston scoring method $^{32}$ with a threshold of 90 Hounsfield units to compensate for nongated imaging.

Reproducibility of echocardiography and CT assessments was determined in two subsets of 20 patients, as described elsewhere. ${ }^{33}$ Coefficients of reproducibility ${ }^{34}$ for aortic-jet velocity and aorticvalve calcium score were $0.32 \mathrm{~m}$ per second and $0.07 \log$ arbitrary units (AU), respectively. ${ }^{33}$

\section{STATISTICAL ANALYSIS}

The two primary end points were progression of stenosis, determined according to changes in aorticjet velocity on Doppler echocardiography, and progression of valvular calcification, as measured by CT. Secondary end points were a composite of clinical end points (death from cardiovascular causes, aortic-valve replacement, or hospitalization attributable to severe aortic stenosis), aortic-valve replacement, death from any cause, hospitalization for any cause, and hospitalization for cardiovascular causes. On the basis of standard deviations of $0.32 \mathrm{~m}$ per second per year ${ }^{8,29,35}$ and $1100 \mathrm{AU}$ per year, ${ }^{32}$ we calculated that the planned sample size of 75 patients per group would give the study a power of 80 percent at a 5 percent significance level to detect a difference in the primary end points of $0.15 \mathrm{~m}$ per second per year in aortic-jet velocity and $500 \mathrm{AU}$ per year in aortic-valve calcium score. These differences are equivalent to a reduction of more than 30 percent in the rate of progression of aortic stenosis. This would exclude a clinically significant effect in the majority of older patients with established disease, although a smaller effect may be clinically relevant in younger patients with mild aortic stenosis.

The data-monitoring committee conducted two interim assessments of safety and an interim assessment of efficacy one year after enrollment began. The trial was to be terminated early in the event of a negative effect of treatment (i.e., $\mathrm{P}<0.05$ ) or a strong benefit of treatment (i.e., $\mathrm{P}<0.001$ ). On the recommendation of the data-monitoring committee, the trial continued until the study was completed.

Analyses were performed using SPSS software, version 12.0, and SAS software, version 8e. Intention-to-treat analyses were used for all clinical outcome variables. Disease progression was deter- 


\begin{tabular}{|c|c|c|}
\hline Characteristic & Atorvastatin ( $N=77$ ) & Placebo $(\mathrm{N}=78$ ) \\
\hline Age $-y r$ & $68 \pm 11$ & $68 \pm 10$ \\
\hline Male sex - \% & 68 & 72 \\
\hline Hypertension - no. & 48 & 54 \\
\hline Hyperlipidemia - no. & 8 & 5 \\
\hline Diabetes mellitus - no. & 3 & 4 \\
\hline Current smoker — no. & 21 & 22 \\
\hline Coronary heart disease - no. & 18 & 21 \\
\hline Cerebrovascular disease - no. & 9 & 11 \\
\hline Peripheral vascular disease - no. & 5 & 13 \\
\hline \multicolumn{3}{|l|}{ Drug history - no. } \\
\hline Aspirin & 43 & 40 \\
\hline ACE inhibitor & 12 & 14 \\
\hline Beta-blocker & 21 & 27 \\
\hline Warfarin & 8 & 12 \\
\hline Height $-\mathrm{cm}$ & $168 \pm 9$ & $169 \pm 8$ \\
\hline Weight - kg & $79 \pm 15$ & $80 \pm 15$ \\
\hline Heart rate $-b p m$ & $68 \pm 11$ & $66 \pm 12$ \\
\hline Systolic blood pressure $-\mathrm{mm} \mathrm{Hg}$ & $144 \pm 18$ & $144 \pm 21$ \\
\hline Diastolic blood pressure $-\mathrm{mm} \mathrm{Hg}$ & $82 \pm 10$ & $81 \pm 12$ \\
\hline \multicolumn{3}{|l|}{ Biochemistry } \\
\hline Total cholesterol - mg/dl & $220 \pm 38$ & $217 \pm 34$ \\
\hline LDL cholesterol - mg/dl & $137 \pm 34$ & $133 \pm 30$ \\
\hline Cholesterol:HDL ratio & $4.1 \pm 1.1$ & $4.1 \pm 1.4$ \\
\hline Urea - mg/dl & $38 \pm 13$ & $43 \pm 13$ \\
\hline Creatinine $-\mathrm{mg} / \mathrm{dl}$ & $1.07 \pm 0.25$ & $1.08 \pm 0.26$ \\
\hline Glucose $-\mathrm{mg} / \mathrm{dl}$ & $91 \pm 19$ & $95 \pm 21$ \\
\hline Sinus rhythm - \% & 94 & 92 \\
\hline Atrial fibrillation - \% & 6 & 8 \\
\hline $\begin{array}{l}\text { Romhilt-Estes score - } \\
\text { median (interquartile range) }\end{array}$ & $1(0-3)$ & $2(1-4)$ \\
\hline Tricuspid aortic valve — \% & 96 & 97 \\
\hline Bicuspid aortic valve — \% & 4 & 3 \\
\hline Aortic.jet velocity $-\mathrm{m} / \mathrm{sec}$ & $3.39 \pm 0.62$ & $3.45 \pm 0.67$ \\
\hline Peak gradient $-\mathrm{mm} \mathrm{Hg}$ & $47.8 \pm 17.4$ & $49.5 \pm 19.5$ \\
\hline Aortic-valve area $-\mathrm{cm}^{2}$ & $1.03 \pm 0.4$ & $1.02 \pm 0.41$ \\
\hline $\begin{array}{l}\text { Aortic-valve calcium score - } \\
\text { median AU (interquartile range) }\end{array}$ & $5424(2750-9689)$ & $6221(3037-9575)$ \\
\hline $\begin{array}{l}\text { Log aortic-valve calcium score - } \\
\qquad \log \mathrm{AU}\end{array}$ & $3.7 \pm 0.5$ & $3.7 \pm 0.6$ \\
\hline
\end{tabular}

* Plus-minus values are means $\pm S D$. ACE denotes angiotensin-converting enzyme, LDL low-density lipoprotein, HDL high-density lipoprotein, and AU arbitrary units.

$\uparrow$ To convert values for cholesterol to millimoles per liter, multiply by 0.02586 . To convert values for urea to millimoles per liter, multiply by 0.357 . To convert va ues for creatinine to micromoles per liter, multiply by 88.4 . To convert values for glucose to millimoles per liter, multiply by 0.05551 . mined primarily by dividing the change between the baseline and final scans by the duration of follow-up. Treatment comparisons for the continuous outcome variables were based on an analysis of covariance, with the prerandomization level of a variable used as a covariate. In a confirmatory analysis of the primary end points, random-coefficient models were fitted to incorporate all observations. ${ }^{36}$ In the subgroup analyses, interaction terms between treatment and subgroup have been added to a model incorporating prerandomization level, treatment, and subgroup to identify factors that were associated with a differential treatment effect within subgroups. Categorical variables have been analyzed using Fisher's exact test. Two-tailed tests were used throughout. Two-sided P values of less than 0.05 were considered to indicate statistical significance.

\section{RESULTS}

Seventy-seven patients were assigned to atorvastatin and 78 to placebo, with a median follow-up of 25 months (range, 7 to 36). As a consequence of minimization, baseline characteristics were well matched (Table 1). Mean aortic-jet velocity was $3.43 \pm 0.64 \mathrm{~m}$ per second (range, 2.5 to 5.0 ), and the median aortic-valve calcium score was $5920 \mathrm{AU}$ (interquartile range, 2485 to 14,231 ). Of the 155 patients, 119 had mild-to-moderate aortic stenosis (aortic-jet velocity, 2.5 to $3.9 \mathrm{~m}$ per second), and 36 had severe stenosis (aortic-jet velocity, $24.0 \mathrm{~m}$ per second).

\section{SERUM CHOLESTEROL CONCENTRATIONS}

The mean serum low-density lipoprotein (LDL) cholesterol concentration remained at $130 \pm 30 \mathrm{mg}$ per deciliter $(3.4 \pm 0.8 \mathrm{mmol}$ per liter) in the placebo group and decreased by 53 percent to $63 \pm 23 \mathrm{mg}$ per deciliter $(1.7 \pm 0.6 \mathrm{mmol}$ per liter $)$ in the atorvastatin group $(\mathrm{P}<0.001)$ (Fig. $1 \mathrm{C})$. Serum total cholesterol was $209 \pm 35 \mathrm{mg}$ per deciliter $(5.5 \pm 0.9 \mathrm{mmol}$ per liter) and $132 \pm 27 \mathrm{mg}$ per deciliter $(3.5 \pm 0.7$ $\mathrm{mmol}$ per liter) in the placebo and atorvastatin groups, respectively $(P<0.001)$, and is in keeping with 97 percent adherence to the study treatment in both groups, which was confirmed by a pill count.

EFFECT OF ATORVASTATIN

ON DISEASE PROGRESSION

Intensive lipid-lowering therapy with $80 \mathrm{mg}$ of atorvastatin daily had no effect on the rate of change in 
Figure 1. Progression in Aortic-Valve Stenosis and Serum LDL Cholesterol Concentrations in Patients Treated with Intensive Atorvastatin Therapy or Matched Placebo.

Patients received $80 \mathrm{mg}$ of atorvastatin daily or matched placebo. LDL denotes low-density lipoprotein, CT computed tomography, and AU arbitrary units. I bars indicate SDs.

aortic-jet velocity or valvular calcification (Table 2). Progression in valvular calcification was $22.3 \pm 21.0$ percent per year in the atorvastatin group, and $21.7 \pm 19.8$ percent per year in the placebo group $(\mathrm{P}=0.93$; ratio of post-treatment aortic-valve calcium score, $0.998 ; 95$ percent confidence interval, 0.947 to 1.050 ). We also performed a longitudinal analysis of the rate of change over time for the two treatment groups with the use of a mixed-effects linear model. ${ }^{36}$ This showed no difference in the rate of disease progression, with point estimates and 95 percent confidence intervals for the treatment difference that were similar to those shown in Table 2 (mean difference in the rate of change of aortic-jet velocity [the change in the atorvastatin group minus that in the placebo group], $0.008 \mathrm{~m}$ per second per year [ -0.058 to 0.075$]$; mean difference in rate of change of aortic-valve calcium score, 71 $\mathrm{AU}$ per year [ -524 to 666 ]). Serum $\mathrm{LDL}$ cholesterol concentrations did not correlate with disease progression demonstrated on echocardiography $(\mathrm{r}=0.021, \mathrm{P}=0.81)$ or $\mathrm{CT}(\mathrm{r}=-0.109, \mathrm{P}=0.21)$. The proportion of patients reaching secondary clinical end points seemed to be less in the atorvastatin group, but none of the comparisons achieved statistical significance (Table 3).

\section{SUBGROUP ANALYSES}

Prespecified subgroup analysis of the primary endpoint data was conducted in patients with mild-tomoderate aortic stenosis (aortic-jet velocity, $<4.0 \mathrm{~m}$ per second) and severe aortic stenosis (aortic-jet velocity, $24.0 \mathrm{~m}$ per second) at baseline. As anticipated from earlier studies, patients with severe stenosis at baseline progressed more rapidly $(\mathrm{P}=0.04)$, but the study findings were consistent regardless of the severity of stenosis at baseline (Table 4).

Likewise, the length of follow-up did not influence outcome. In those followed for more than 24

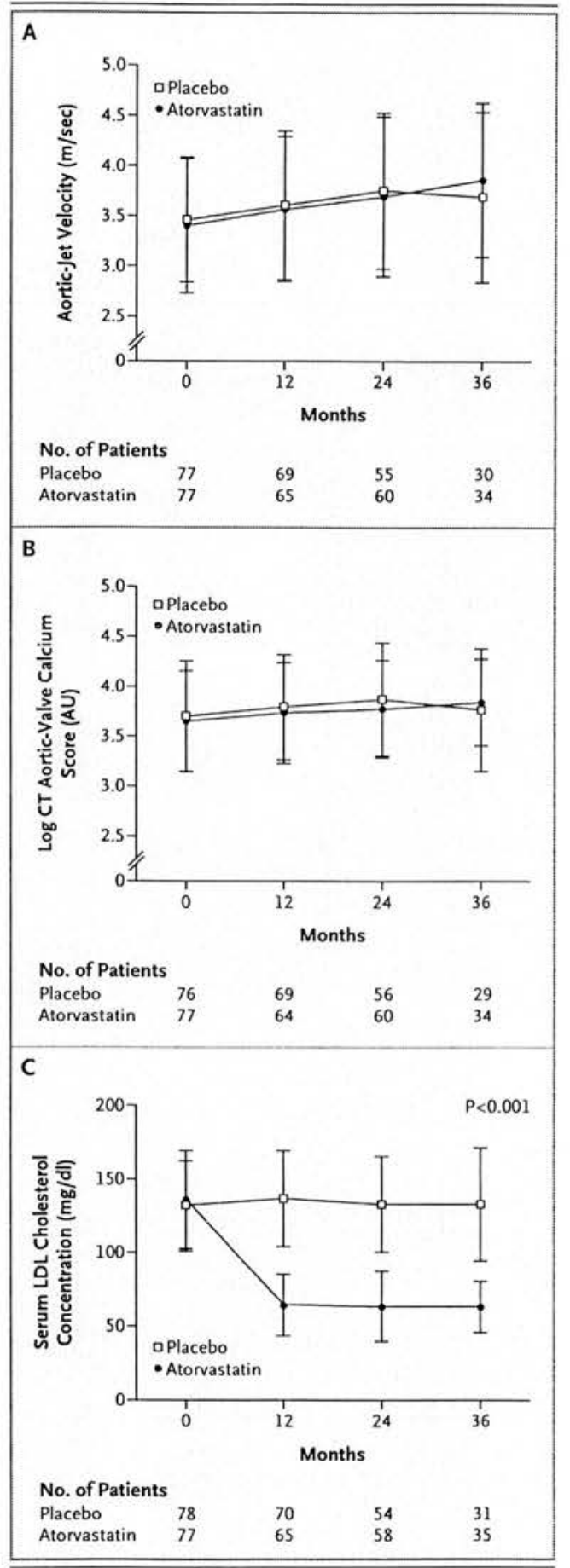




\begin{tabular}{|c|c|c|c|c|c|}
\hline Variable & All Patients & Atorvastatin & Placebo & $\begin{array}{c}\text { Adjusted Difference: } \\
\text { Atorvastatin-Placebo } \\
(95 \% \mathrm{CI})\end{array}$ & P Value \\
\hline \multicolumn{6}{|l|}{ Echocardiography } \\
\hline No. of patients & 134 & 65 & 69 & & \\
\hline Change in aortic-jet velocity $(\mathrm{m} / \mathrm{sec} / \mathrm{yr})$ & $0.201 \pm 0.208$ & $0.199 \pm 0.210$ & $0.203 \pm 0.208$ & $0.002(-0.066$ to 0.070$)$ & 0.95 \\
\hline Increase in peak gradient $(\mathrm{mm} \mathrm{Hg} / \mathrm{yr})$ & $6.52 \pm 7.24$ & $6.48 \pm 7.43$ & $6.56 \pm 7.10$ & $0.21(-2.02$ to 2.45$)$ & 0.85 \\
\hline Change in aortic-valve area $\left(\mathrm{cm}^{2} / \mathrm{yr}\right)$ & $-0.081 \pm 0.107$ & $-0.079 \pm 0.107$ & $-0.083 \pm 0.107$ & $0.007(-0.026$ to 0.040$)$ & 0.68 \\
\hline \multicolumn{6}{|l|}{ Computed tomography } \\
\hline No. of patients & 133 & 64 & 69 & & \\
\hline $\begin{array}{l}\text { Absolute change in aortic-valve calcium score } \\
(\mathrm{AU} / \mathrm{yr})\end{array}$ & $1608 \pm 1865$ & $1564 \pm 1956$ & $1648 \pm 1790$ & $85(-554$ to 723$)$ & 0.80 \\
\hline Change in log aortic-valve calcium score (per yr) & $0.20 \pm 0.16$ & $0.20 \pm 0.16$ & $0.20 \pm 0.15$ & $0.00(-0.05$ to 0.05$)$ & 0.93 \\
\hline
\end{tabular}

* Plus-minus values are means $\pm \mathrm{SD} . \mathrm{Cl}$ denotes confidence interval, and $\mathrm{AU}$ arbitrary units.

\begin{tabular}{|c|c|c|c|}
\hline Secondary End Point & $\begin{array}{l}\text { Atorvastatin } \\
\qquad(\mathrm{N}=77)\end{array}$ & $\begin{array}{l}\text { Placebo } \\
(\mathrm{N}=78)\end{array}$ & $\begin{array}{c}\text { P Value } \\
\text { (Fisher's } \\
\text { Exact Test) }\end{array}$ \\
\hline Composite* & 13 & 21 & 0.19 \\
\hline Death from cardiovascular causes & 3 & 3 & 1.00 \\
\hline Aortic-valve replacement & 11 & 19 & 0.17 \\
\hline Hospitalization for severe aortic stenosis & 3 & 5 & 0.73 \\
\hline Death from any cause & 3 & 5 & 0.73 \\
\hline Hospitalization for any cause & 10 & 12 & 0.84 \\
\hline
\end{tabular}

* The composite end point was death from cardiovascular causes, aortic-valve replacement, or hospitalization for severe aortic stenosis. the creatine kinase level to more than five times the upper limit of the normal range, without symptoms of myositis; one of these patients was withdrawn at the request of the data-monitoring committee. There were no cases of rhabdomyolysis and no serious adverse events.

\section{DISCUSSION}

In this randomized, double-blind, placebo-controlled, parallel-group trial of lipid-lowering therapy in patients with calcific aortic stenosis, a single coordinating center used a consistent and reproducible approach to assess the severity of aortic stenosis. ${ }^{33}$ We have clearly shown that high-dose atorvastatin reduces serum LDL cholesterol concentrations by more than a factor of two, as anticipated, ${ }^{37}$ but it does not halt the progression or induce regression of the valvular disease process. This was shown with the use of two distinct measures of disease severity - aortic-jet velocity assessed with Doppler echocardiography and valvular calcification assessed with helical CT. Moreover, there was no relationship between serum LDL cholesterol concentrations and the progression of aortic stenosis, nor did high-dose atorvastatin have a demonstrable effect on clinical end points. Thus, regardless of the method of assessing disease progression, we have consistently shown that aortic stenosis progresses despite intensive reductions in serum cholesterol concentrations.

The minimization technique helped ensure that there were no baseline inequalities between the 


\begin{tabular}{|c|c|c|c|c|c|c|c|c|}
\hline \multirow[t]{2}{*}{ Characteristic } & \multicolumn{4}{|c|}{ Atorvastatin } & \multicolumn{4}{|c|}{ Placebo } \\
\hline & No. & $\begin{array}{c}\text { Baseline } \\
\text { Value } \\
\mathrm{m} / \mathrm{sec}\end{array}$ & No. & $\begin{array}{c}\text { Rate } \\
\text { of Change } \\
\mathrm{m} / \mathrm{sec} / \mathrm{\gamma r}\end{array}$ & No. & $\begin{array}{c}\text { Baseline } \\
\text { Value } \\
\mathrm{m} / \mathrm{sec}\end{array}$ & No. & $\begin{array}{c}\text { Rate } \\
\text { of Change } \\
\mathrm{m} / \mathrm{sec} / \mathrm{yr}\end{array}$ \\
\hline \multicolumn{9}{|c|}{ Baseline severity of stenosis $\uparrow$} \\
\hline Mild to moderate & 58 & $3.12 \pm 0.43$ & 49 & $0.17 \pm 0.21$ & 61 & $3.18 \pm 0.44$ & 55 & $0.19 \pm 0.20$ \\
\hline Severe & 19 & $4.24 \pm 0.21$ & 16 & $0.27 \pm 0.21$ & 17 & $4.45 \pm 0.26$ & 14 & $0.27 \pm 0.23$ \\
\hline \multicolumn{9}{|l|}{ Duration of follow-up } \\
\hline$\leq 24 \mathrm{Mot}$ & 30 & $3.49 \pm 0.69$ & 30 & $0.19 \pm 0.22$ & 37 & $3.64 \pm 0.67$ & 37 & $0.23 \pm 0.25$ \\
\hline$>24 \mathrm{Mol}$ & 35 & $3.31 \pm 0.55$ & 35 & $0.21 \pm 0.20$ & 32 & $3.28 \pm 0.61$ & 32 & $0.17 \pm 0.14$ \\
\hline
\end{tabular}

* Plus-minus values are means \pm SD. $P=0.57$ for the interaction of treatment and the baseline severity of stenosis, and $P=0.41$ for the interaction of treatment and the duration of follow-up.

$\uparrow$ Patients with mild-to-moderate aortic stenosis had an aortic-jet velocity of less than $4.0 \mathrm{~m}$ per second, and those with severe stenosis an aortic-jet velocity of at least $4.0 \mathrm{~m}$ per second.

The median follow-up was 23 months.

The median follow-up was 33 months.

treatment groups. Several factors may have influenced our ability to detect an effect of statin therapy on the progression of aortic stenosis in this trial. First, as a consequence of our inclusion criteria, we recruited some patients with severe disease and an aortic-jet velocity of at least $4 \mathrm{~m}$ per second, and it could be argued that lipid-lowering therapy is unlikely to influence disease progression at such an advanced stage. We therefore conducted a prespecified subgroup analysis excluding patients with a baseline aortic-jet velocity of $4 \mathrm{~m}$ per second or more. Our findings were consistent regardless of the severity of stenosis at baseline - atorvastatin had no effect on disease progression, even in the majority of patients with mild-to-moderate stenosis. We excluded patients with an aortic-jet velocity of less than $2.5 \mathrm{~m}$ per second, and we acknowledge that intervening at this earlier stage of the disease process may have been more beneficial. However, such patients do not commonly present to routine clinical practice, and their identification would potentially require population screening.

Second, two years of treatment may not have been sufficient to influence the natural history of the disease. We assessed this possibility by determining if patients with a longer follow-up showed a treatment benefit. In patients who underwent nearly three years of treatment with intensive statin therapy, no trend toward a beneficial effect of atorvastatin was apparent. Therefore, we do not believe that the lack of an effect was due to an inadequate treatment period.
Finally, our study was designed to detect a substantial delay in disease progression and was not powered to assess meaningful effects on clinical end points, such as valve replacement and cardiovascular death. Although we can exclude a treatment benefit of the magnitude previously reported in retrospective observational studies (a reduction in the aortic-jet velocity of $0.30 \mathrm{~m}$ per second per year ${ }^{29}$ and valvular calcification of 30 percent per year $\left.{ }^{24,26}\right)$, the 95 percent confidence intervals indicate that we may have missed a modest treatment benefit (a delay in disease progression of $<0.07 \mathrm{~m}$ per second per year for aortic-jet velocity and $<5$ percent per year for valvular calcification). Although such modest reductions are unlikely to be meaningful in the majority of older patients, a small decrease in disease progression may be clinically important in younger patients with mild disease that may progress over many years.

Given the strength of the data linking aortic stenosis with atherosclerosis and hypercholesterolemia, why have we failed to halt the progression of calcific aortic stenosis? One potential explanation is that, although these features may drive the initiation of aortic stenosis, disease progression may depend on other factors. The aortic valve is subject to continuous dynamic mechanical stress, and the plasticity and structure of the leaflets can have an overriding influence, as is the case with a bicuspid valve. Moreover, in contrast to atherosclerosis, aortic stenosis is associated with a virtual absence of smooth-muscle-cell proliferation and lipid-laden 
macrophages ${ }^{2}$ and is dominated by earlier and more extensive mineralization. Decreasing the lipid pool and strengthening the fibrous cap may be less relevant to the progression of aortic stenosis than they are for the reduction in atherosclerotic-plaque rupture with statin therapy in patients with coronary heart disease.

Because of the association between aortic stenosis and coronary artery disease, statin therapy in patients with aortic stenosis may confer secondary preventive benefits that are independent of its effects on the valvular disease process. The current study was not powered to assess the benefits of lipidlowering therapy on cardiovascular end points such as nonfatal and fatal myocardial infarction. It remains a possibility that aortic stenosis and sclerosis $^{38}$ may be important markers of occult vascular disease and may identify patients who would gain from the preventive benefits of statin therapy.
We conclude that intensive lipid-lowering therapy with $80 \mathrm{mg}$ of atorvastatin daily does not halt the progression of calcific aortic stenosis or induce its regression. Nevertheless, this trial does not rule out a small but potentially relevant reduction in the rate of disease progression or a significant reduction in major clinical end points. Our study reinforces the need for a long-term, large-scale, randomized, controlled trial of intensive lipid-lowering therapy in patients with calcific aortic stenosis, particularly in those with early, mild disease. In the meantime, we do not recommend statin therapy for patients with calcific aortic stenosis in the absence of coexisting vascular disease.

Supported by a grant from the British Heart Foundation (PG/ $2000 / 044$ ), by an educational award from Pfizer, and by the Wellcome Trust Clinical Research Facility, Edinburgh.

Drs. Newby, Bloomfield, and Boon report having received unrestricted educational grant support from Pfizer, and Drs. Newby, Northridge, and Boon report having received consulting fees from and having served on advisory boards for Pfizer.

\section{APPENDIX}

The following participated in the Scottish Aortic Stenosis and Lipid Lowering Trial, Impact on Regression (SALTIRE): Research team: L. Anderson, C. Bell, M. Bland, J. Burton, S. Cameron, N. Cruden, J. Cunningham, H. Cuthbertson, L. Flint, M. Henderson, D. Lyle, M. O'Donnell, F. Paterson, K. Paterson, S. Robinson, H. Spence, J. Tickner, A. White. Collaborating centers (all in the United Kingdom): Borders General Hospital, Melrose - P. Broadhurst, C. Norris, P. Leslie, J. Gaddie; Eastem General Hospital, Edinburgh - A. Elder; Royal Infirmary, Edinburgh - K. Fox, N. Grubb, A. Flapan, H. Miller, N. Uren; Falkirk and District Royal Infirmary, Falkirk - A. Hargreaves, P. McSorely; Queen Margare Hospital, Dunfermline - D. MacLeod; Roodland's Hospital, Haddington - A. Flapan; St. Johns' Hospital, Livingston - J. Irving, A. Jacob; Royal Infirmary, Stirling - A. Bridges, S. Glen; Wellcome Trust Clinical Research Facility, Edinburgh; Western General Hospital, Edinburgh - M. Denvir, T. Shaw, I. Starkey. Pharmacy: Royal Infirmany, Edinburgh - B. Booth; Freeman Hospital, Newcastle-upon-Tyne, United Kingdom - A. Heed. Medical Statistics: University of Edinburgh, Edinburgh - T. Forster.

\section{REFERENCES}

1. Stewart BF, Siscovick D, Lind BK, et al. Clinical factors associated with calcific aortic valve disease: Cardiovascular Health Study. J Am Coll Cardiol 1997;29:630-4.

2. Otto CM, Kuusisto J, Reichenbach DD, Gown AM, O'Brien KD. Characterization of the early lesion of 'degenerative' valvular aortic stenosis: histological and immunohistochemical studies. Circulation 1994;90: 844-53.

3. Olsson $M$, Dalsgaard $C$, Haegerstrand A, Rosenquist M, Rydén L, Nilsson J. Accumulation of $\mathrm{T}$ lymphocytes and expression of interleukin-2 receptors in nonrheumatic stenotic aortic valves. J Am Coll Cardiol 1994;23:1162-70.

4. O'Brien KD, Reichenbach DD, Marcovina SM, Kuusisto J, Alpers CE, Otto CM. Apolipoproteins B, (a), and E accumulate in the morphologically early lesion of 'degenerative' valvular aortic stenosis. Arterioscler Thromb Vasc Biol 1996;16:523-32.

5. Olsson $M$, Thyberg J, Nilsson J. Presence of oxidized low density lipoprotein in nonrheumatic stenotic aortic valves. Arterioscler Thromb Vasc Biol 1999;19:121822.

6. Mautner GC, Roberts WC. Reported fre- quency of coronary arterial narrowing by angiogram in patients with valvular aortic stenosis. Am J Cardiol 1992;70:539-40. 7. Peltier M, Trojette F, Sarano ME, Grigioni F, Slama MA, Tribouilloy CM. Relation between cardiovascular risk factors and nonrheumatic severe calcific aortic stenosis among patients with a three-cuspid aortic valve. Am J Cardiol 2003;91:97-9.

8. Otto CM, Burwash IG, Legget ME, et al. Prospective study of asymptomatic valvula aortic stenosis: clinical, echocardiographic, and exercise predictors of outcome. Circula tion 1997; 95:2262-70.

9. Davies SW, Gershlick AH, Balcon R. Progression of valvar aortic stenosis: a long term retrospective study. Eur Heart J 1991 12:10-4.

10. Bahler RC, Desser DR, Finkelhor RS, Brener SJ, Youssefi M. Factors leading to progression of valvular aortic stenosis. Am J Cardiol 1999;84:1044-8.

11. Rosenhek R, Binder T, Porenta G, et al. Predictors of outcome in severe, asymptomatic aortic stenosis. N Engl J Med 2000;343 611-7.

12. Palta S, Pai AM, Gill KS, Pai RG. New insights into the progression of aortic stenosis implications for secondary prevention. Circulation 2000;101:2497-502.

13. Nassimiha D, Aronow WS, Ahn C, Goldman ME. Association of coronary risk factors with progression of valvular aortic stenosis in older persons. Am J Cardiol 2001;87:1313-4

14. Rallidis L, Naoumova RP, Thompson GR, Nihoyannopoulos P. Extent and severity of atherosclerotic involvement of the aortic valve and root in familial hypercholesterolaemia. Heart 1998;80:583-90.

15. Keller C, Schmitz H, Theisen K, Zollner $\mathrm{N}$. Regression of valvular aortic stenosis due to homozygous familial hypercholesterolemia following plasmapheresis. Klin Wochenschr 1986;64:338-41.

16. Shepherd J, Cobbe SM, Ford I, et al. Prevention of coronary heart disease with pravastatin in men with hypercholesterolemia. N Engl J Med 1995;333:1301-7.

17. Heart Protection Study Collaborative Group. MRC/BHF Heart Protection Study of cholesterol lowering with simvastatin in 20,536 high-risk individuals: a randomised placebo-controlled trial. Lancet 2002;360:7 22.

18. Zhao XQ, Brown BG, Hillger L, et al. Ef- 
fects of intensive lipid-lowering therapy on the coronary arteries of asymptomatic subjects with elevated apolipoprotein B. Circulation 1993;88:2744-53.

19. Pitt B, Mancini GB, Ellis SG, Rosman HS, Park JS, McGovern ME. Pravastatin limitation of atherosclerosis in the coronary arteries (PLAC I): reduction in atherosclerosis progression and clinical events. J Am Coll Cardiol 1995;26:1133-9.

20. Jukema JW, Bruschke AV, van Boven AJ, et al. Effects of lipid lowering by pravastatin on progression and regression of coronary artery disease in symptomatic men with normal to moderately elevated serum cholesterol levels: the Regression Growth Evaluation Statin Study (REGRESS). Circulation 1995; 91:2528-40.

21. Callister TQ Raggi P, Cooil B, Lippolis NJ, Russo DJ. Effect of HMG-CoA reductase inhibitors on coronary artery disease as assessed by electron-beam computed tomography. N Engl J Med 1998;339:1972-8.

22. Budoff MJ, Lane KL, Bakhsheshi H, et al. Rates of progression of coronary calcium by electron beam tomography. Am J Cardiol 2000;86:8-11.

23. Achenbach S, Ropers D, Pohle K, et al. Influence of lipid-lowering therapy on the progression of coronary artery calcification: a prospective evaluation. Circulation 2002; 106:1077-82.

24. Pohle K, Maffert R, Ropers D, et al. Progression of aortic valve calcification: association with coronary atherosclerosis and car- diovascular risk factors. Circulation 2001; 104:1927-32.

25. Novaro GM, Tiong IY, Pearce GL, Lauer MS, Sprecher DL, Griffin BP. Effect of hydroxymethylglutaryl coenzyme $A$ reductase inhibitors on the progression of calcific aortic stenosis. Circulation 2001;104:2205-9.

26. Shavelle DM, Takasu J, Budoff MJ, Mao $S$, Zhao XQ, O'Brien KD. HMG CoA reductase inhibitor (statin) and aortic valve calcium. Lancet 2002-359:1125-6.

27. Aronow WS, Ahn C, Kronzon I, Goldman ME. Association of coronary risk factors and the use of statins with progression of mild valvular aortic stenosis in older persons. Am J Cardiol 2001;88:693-5.

28. Bellamy MF, Pellikka PA, Klarich KW, Tajik AJ, Enriquez-Sarano M. Association of cholesterol levels, hydroxymethylglutaryl coenzyme-A reductase inhibitor treatment, and progression of aortic stenosis in the community. J Am Coll Cardiol 2002;40: 1723-30.

29. Rosenhek R, Rader F, Loho N, et al. Statins but not angiotensin-converting enzyme inhibitors delay progression of aortic stenosis. Circulation 2004;110:1291-5.

30. Zoghbi WA, Enriquez-Sarano M, Foster $\mathrm{E}$, et al. Recommendations for evaluation of the severity of native valvular regurgitation with two-dimensional and Doppler echocardiography. J Am Soc Echocardiogr 2003; 16:777-802.

31. Treasure T, MacRae KD. Minimisation: the platinum standard for trials? Random- isation doesn't guarantee similarity of groups: minimisation does. BMJ 1998;317: 362-3.

32. Shemesh J, Apter S, Rozenman J, et al. Calcification of coronary arteries: detection and quantification with double-helix CT. Radiology 1995; 197:779-83.

33. Cowell SJ, Newby DE, Burton J, et al. Aortic valve calcification on computed tomography predicts the severity of aortic stenosis. Clin Radiol 2003:58:712-6.

34. Bland JM, Altman DG. Statistical methods for assessing agreement between two methods of clinical measurement. Lancet 1986;1:307-10.

35. Faggiano P, Ghizzoni G, Sorgato A, et al. Rate of progression of valvular aortic stenosis in adults. Am J Cardiol 1992;70:22933.

36. Brown H, Prescott RJ. Applied mixed models in medicine. Chichester, England: John Wiley, 1999:239-41.

37. Jones P, Kafonek S, Laurora I, Hunninghake D. Comparative dose efficacy study of atorvastatin versus simvastatin, pravastatin, lovastatin, and fluvastatin in patients with hypercholesterolemia (the CURVES study). Am J Cardiol 1998;81:582-7. [Erratum, Am ] Cardiol 1998;82:128.]

38. Otto CM, Lind BK, Kitzman DW, Gersh BJ, Siscovick DS. Association of aortic-valve sclerosis with cardiovascular mortality and morbidity in the elderly. N Engl J Med 1999; 341:142-7.

Copyright 12005 Massachusetts Medical Society

\section{CLINICAL TRIAL REGISTRATION}

The Journal encourages investigators to register their clinical trials in a public trials registry. The members of the International Committee of Medical Journal Editors plan to consider clinical trials for publication only if they have been registered

(see N Engl J Med 2004;351:1250-1). The National Library of Medicine's www.clinicaltrials.gov is a free registry, open to all investigators, that meets the committee's requirements. 


\title{
CARDIOVASCULAR MEDICINE
}

\section{Progressive coronary calcification despite intensive lipid-lowering treatment: a randomised controlled trial}

\author{
E S Houslay, S J Cowell, R J Prescott, J Reid, J Burton, D B Northridge, N A Boon, \\ D E Newby, on behalf of the Scottish Aortic Stenosis and Lipid Lowering Therapy, \\ Impact on Regression (SALTIRE) trial Investigators
}

See end of article for authors' affiliations

Correspondence to: Professor David E Newby SU.314 Chancellor's Building, University of Edinburgh, Edinburgh EHI6 4SA, UK; d.e. newby@ed.ac.uk

Accepted 17 January 2006 Published Online First 31 January 2006
Heart 2006:92:1207-1212. doi: 10.1136/hrt.2005.080929

Objectives: To evaluate the effect of intensive lipid-lowering treatment on coronary artery calcification in a substudy of a trial recruiting patients with calcific aortic stenosis.

Methods: In a double blind randomised controlled trial, 102 patients with calcific aortic stenosis and coronary artery calcification were randomly assigned by the minimisation technique to atorvastatin $80 \mathrm{mg}$ daily or matched placebo. Coronary artery calcification was assessed annually by helical computed tomography.

Results: 48 patients were randomly assigned to atorvastatin and 54 to placebo with a median follow up of 24 months (interquartile range 24-30). Baseline characteristics and coronary artery calcium scores were similar in both groups. Atorvastatin reduced serum low density lipoprotein cholesterol $(-53 \%, \mathrm{p}<0.001)$ and $C$ reactive protein $(-49 \%, p<0.001)$ concentrations whereas there was no change with placebo $(-7 \%$ and $17 \%, p>0.95$ for both). The rate of change in coronary artery calcification was $26 \% /$ year (0.234 (SE 0.037) log arbitrary units $(\mathrm{AU}) /$ year; $n=39$ ) in the atorvastatin group and $18 \% /$ year $(0.167$ (SE 0.034 ) $\log \mathrm{AU} /$ year; $n=49$ ) in the placebo group, with a geometric mean difference of $7 \% /$ year $195 \%$ confidence interval $-3 \%$ to $18 \%, p=0.18$ ). Serum low density lipoprotein concentrations were not correlated with the rate of progression of coronary calcification $(r=0.05, p=0.62)$.

Conclusion: In contrast to previous observational studies, this randomised controlled trial has shown that, despite reducing systemic inflammation and halving serum low density lipoprotein cholesterol concentrations, statin treatment does not have a major effect on the rate of progression of coronary artery calcification.

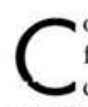
oronary artery calcification is an independent risk factor for coronary heart disease, with even low coronday calcium scuics duubling the risk of coronary events.' The relative tisk associatcd with coronary calcifica tion is greater than that associated with established factors such as smoking, hypertension and diabetes mellitus. Progression of coronary artery calcification is associated with a higher incidence of coronary events even among people who are asymptomatic at the time of initial scanning. ${ }^{2}$ Thus, not only is the presence of coronary artery calcification indicative of atheromatous plaque disease but its progression may correspond with cardiovascular event rates.

Statin treatment has a proven role in the primary" " and secondary prevention ${ }^{5-1}$ of cardiovascular disease, with incremental benefits seen with more intensive reductions in serum cholesterol concentrations. ${ }^{8}$ Previous studies ${ }^{10}$ have reported that statins can halt the progression and may even induce regression of coronary artery calcification. Indeed, the rate of progression of coronary artery calcification correlates with the average serum low density lipoprotein (LDL) cholesterol concentration. " This has led to the use of computed tomography to monitor disease progression and response to treatment, particularly with statins. Two recent trials, however, did not show a benefit of statin on the progression of coronary artery calcification in asymptomatic people." 12

The SALTIRE (Scottish Aortic Stenosis Lipid lowering Therapy, Impact on Regression) trial was a prospective double blind, randomised controlled study of intensive lipid-lowering treatment of patients with calcific aortic stenosis." As part of this trial, aortic valve and coronary artery calcium scores are measured by helical computed tomography. The objective of this substudy was to assess the effect of atorvastatin $80 \mathrm{mg}$ daily on the rate of progression of coronary artery calcification in patients with calcific aortic stenosis.

\section{METHODS}

\section{Patient population}

Patients aged $>18$ years with calcific aortic stenosis (grade 1-3 calcification on echocardiography ${ }^{14}$ ) and a peak post valve velocity of $\geqslant 2.5 \mathrm{~m} / \mathrm{s}$ were recruited from eight hospital centres across the southeast of Scotland. Exclusion criteria were women of childbearing potential without contraception, active or chronic liver disease, history of alcohol or drug misuse, severe mitral stenosis (valve area $<1 \mathrm{~cm}^{2}$ ), severe mitral or aortic regurgitation, ${ }^{15}$ major left ventricular dysfunction (ejection fraction $<35 \%$ ), planned aortic valve replacement, intolerance to statins, patients who were taking or would in the opinion of the treating physician benefit from statins, baseline serum total cholesterol of $<4.0 \mathrm{mmol} / \mathrm{l}$, and permanent pacemaker or cardiodefibrillator. For the substudy, we also excluded patients who had no coronary artery calcification on computed tomography. The study was conducted with the approval of all the regional research ethics committees and in accordance with the Declaration of

Abbreviations: AU, arbitrary units; BELLES, Beyond Endorsed Lipid lowering with EBT Scanning: CRP, C reactive protein; LDL, low density lipoprotein; SALTIRE, Scottish Aortic Stenosis Lipid Lowering Therapy, Impact on Regression 
Helsinki. Written informed consent was obtained from each participant.

\section{Study protocol}

Between March 2001 and April 2002, the blinded study coordinator randomly assigned eligible patients by the minimisation technique ${ }^{16}$ with a dedicated locked computer program (Edinburgh University), which incorporated eight baseline variables: age, sex, smoking habit, hypertension, diabetes mellitus, serum cholesterol concentration, peak aortic jet velocity and aortic calcium score. Patients were assigned either to atorvastatin $80 \mathrm{mg}$ daily or matched placebo (Pfizer, Tadworth, UK) as a single daily dose in numbered containers.

Patients were assessed at baseline, two months, six months and every six months thereafter for a minimum of two years. Clinical evaluation included assessment of functional status, adverse events and biochemical blood analysis. Serum high sensitivity C reactive protein (CRP) concentrations were determined by a highly sensitive immunonephelometric method (Dade Behring, Milton Keynes, UK) as previously described..$^{17}$ All patients underwent computed tomography within the month before randomisation to study treatment and at each annual visit. Randomly assigned patients who were later treated with an open label statin by their attending physician were immediately scanned and withdrawn from further observation.

\section{Computed tomography}

A single blinded operator performed computed tomography with a double helix scanner (Twin II Flash; Philips Medical Systems (UK), Stevenage, UK) calibrated against a standard phantom. Images were acquired in $2.7 \mathrm{~mm}$ slices (with a $0.75 \mathrm{~s}$ full $360^{\circ}$ scan mode) through the region of the coronary arteries with a pitch of 0.7 and an increment of $1.3 \mathrm{~mm}$ during held inspiration. Exposure factors were $120 \mathrm{kV}$ at $270 \mathrm{~mA}$ and the scan angle was $360^{\circ}$. Images were analysed off line with an automated, computerised software program (Picker cardiac scoring). This uses an Agatston scoring method, ${ }^{18}$ producing sensitivity and specificity comparable with electron beam computed tomography. ${ }^{17}$ Scans were scored by both the Agatston (130 HU threshold) and the modified Agatston (90 HU threshold) methods. ${ }^{19}$ The Agatston method has been shown to reduce interobserver and interscan variation compared with the threshold of $90 \mathrm{HU} .{ }^{20} \mathrm{To}$ assess the reproducibility of the method, repeated baseline computed tomography scans were recorded within four weeks of each other in an unselected random sample of 16 patients.

\section{Data analysis and statistics}

Coronary artery calcium scores are expressed in arbitrary units (AU) based on the $130 \mathrm{HU}$ threshold. The calcium scores and high sensitivity CRP concentrations were not normally distributed and data are presented as median

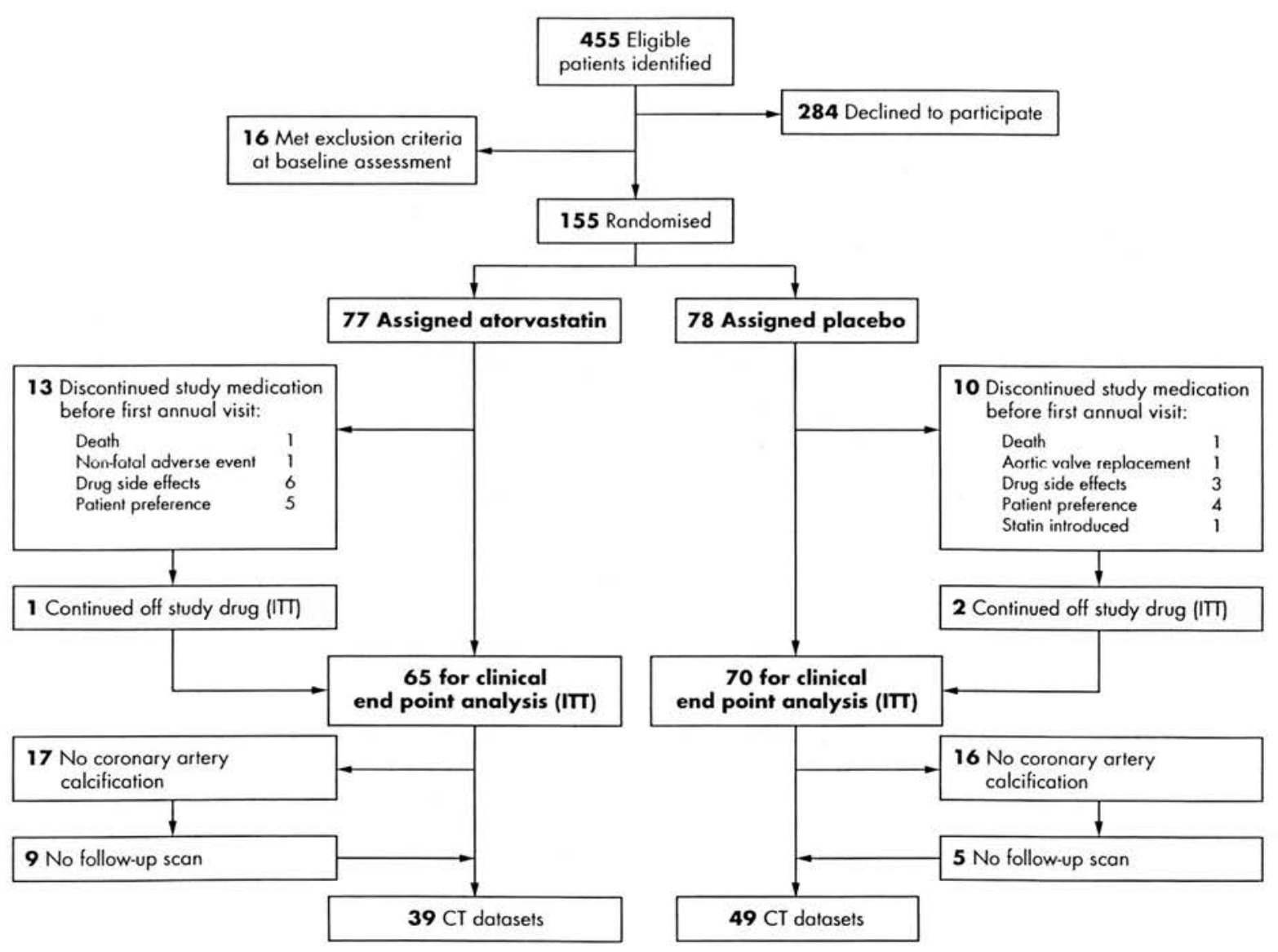

Figure 1 Consolidated Standards of Reporting Trials (CONSORT) flow diagram of patients recruited into the trial and substudy. CT, computed tomography; ITT, intention to treat. 


\begin{tabular}{|c|c|c|}
\hline Characteristic & $\begin{array}{l}\text { Atorvastatin } \\
(n=39)\end{array}$ & $\begin{array}{l}\text { Placebo } \\
(n=49)\end{array}$ \\
\hline Age (years) & $70(8)$ & $70(9)$ \\
\hline Men & $74 \%$ & $78 \%$ \\
\hline \multirow{2}{*}{\multicolumn{3}{|c|}{$\begin{array}{l}\text { Body mass index }\left(\mathrm{kg} / \mathrm{m}^{2}\right) \\
\text { Cardiovascular risk factors }\end{array}$}} \\
\hline & & \\
\hline Hypertension & 22 & 28 \\
\hline Hyperlipidaemia & 3 & 2 \\
\hline Diabetes mellitus & 0 & 2 \\
\hline Current smoker & 5 & 10 \\
\hline \multicolumn{3}{|l|}{ Cardiovascular disease } \\
\hline Coronary heart disease & 7 & 13 \\
\hline Cerebrovascular disease & 5 & 7 \\
\hline Peripheral vascular disease & 3 & 7 \\
\hline \multicolumn{3}{|l|}{ Drug history } \\
\hline Aspirin & 17 & 26 \\
\hline ACE inhibitor & 7 & 8 \\
\hline$\beta$ blocker & 11 & 15 \\
\hline Worfarin & 4 & 8 \\
\hline \multicolumn{3}{|l|}{ Blood pressure $(\mathrm{mm} \mathrm{Hg})$} \\
\hline Systolic & $143(18)$ & $140(19)$ \\
\hline Diastolic & 82 (11) & 78 (11) \\
\hline \multicolumn{3}{|l|}{ Lipid profile } \\
\hline Total cholesterol $(\mathrm{mmol} / \mathrm{l})$ & $5.7(0.9)$ & $5.5(0.9)$ \\
\hline LDL cholesterol (mmol/I) & $3.6(0.8)$ & $3.4(0.7)$ \\
\hline HDL cholesterol (mmol/i) & $1.5(0.4)$ & $1.5(0.4)$ \\
\hline Total cholesterol:HDL & $4.2(1.2)$ & $4.0(1.0)$ \\
\hline Triglycerides (mmol/l) & $1.5(0.8)$ & $1.4(0.7)$ \\
\hline \multicolumn{3}{|l|}{ Coronary calcification score (AU) } \\
\hline Left anterior descending artery & $112(40-285)$ & $207(76-461)$ \\
\hline Gircumflex ortery & $0(0-9)$ & $0(0-4)$ \\
\hline Right coronary artery & $0(0-29)$ & $0(0-0)$ \\
\hline Total coronary score & $195(57-448)$ & $235(83-526)$ \\
\hline $\log$ total coronary score $(\log A U)$ & $2.16(0.68)$ & $2.30(0.65)$ \\
\hline
\end{tabular}

(interquartile range) or mean (SD) after logarithmic transformation $(\log \mathrm{AU})$. The primary end point, the rate of change of coronary calcium scores, was analysed with random coefficient models ${ }^{1321}$ after logarithmic transformation of the scores. In summarising the data, we calculated the change in coronary artery calcium scores by dividing the change between the baseline and final scores by the duration of follow up. Rate of change in coronary calcium score is expressed as percentage change per year or as absolute change in the logarithm of the coronary artery calcium score. Reproducibility was assessed by the method of Bland and Altman." As well as tests of significance, 95\% confidence intervals are reported as appropriate. Significance was taken as a two-sided $\mathrm{p}<0.05$.

\section{RESULTS}

Of 155 patients recruited into the SALTIRE trial, 102 had coronary calcification at baseline (fig 1), of whom 88 had at least two scans. Coronary calcification predominated in the left anterior descending artery ( $100 \%$ of patients) although it was also present in the circumflex $(33 \%)$ and right $(27 \%)$ coronary arteries. Baseline characteristics and coronary artery calcium scores were well matched in both treatment groups (table 1 ) in the 88 evaluable participants.

\section{Reproducibility}

The reproducibility of the left anterior descending coronary score and of the total coronary score was examined with the approach of Bland and Altman." Without transformation, the difference between replicate observations tended to increase with the magnitude of the measurement. After logarithmic transformation, higher values showed stable
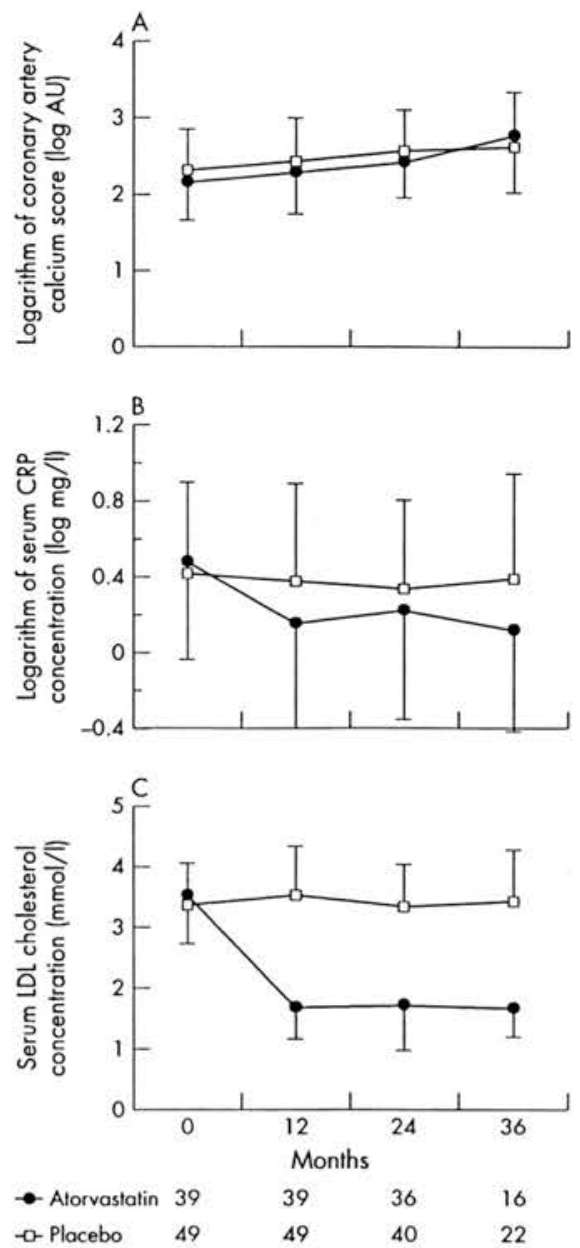

Figure 2 Progression of (A) coronary artery calcification, (B) serum C reactive protein $(C R P)$ concentrations $(p<0.001$, atorvastatin $v$ placebo) and (C) serum low density lipoprotein (LD) cholesterol concentrations ( $p<0.001$, atorvastatin $v$ placebo) in patients treated with atorvastatin $80 \mathrm{mg}$ daily or matched placebo. AU, arbitrary units.

differences, but differences were higher at the lowest scores. Overall, the differences on the log scale correspond to a coefficient of variation of $28 \%$ for both variables, but when the analysis was restricted to the 10 pairs with a geometric mean score above 100 , the coefficient of variation was $10 \%$ for both variables.

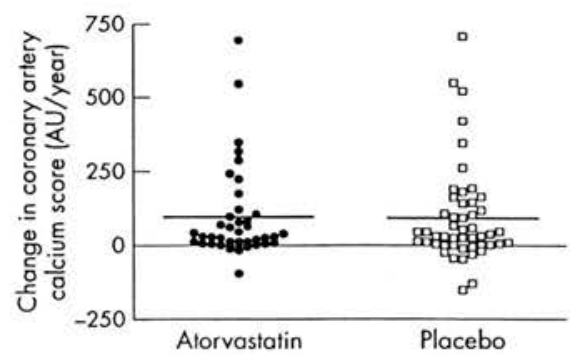

Figure 3 Absolute rate of change in coronary calcium score expressed in arbitrary units (AU) per year for patients treated with atorvastatin 80 mg or matched placebo. 


\section{Effect of atorvastatin treatment}

Patients were followed up for a median of 24 months (interquartile range $24-30$ ). Atorvastatin $80 \mathrm{mg}$ daily more than halved serum LDL cholesterol concentrations (53 (SD $19) \%, p<0.001$ ), whereas placebo had no effect (fig 2 ). This reduction in serum LDL cholesterol concentrations was associated with a major decrease in serum CRP concentrations from 1.95 (interquartile range $1.15-4.86$ ) to $1.00 \mathrm{mg} / \mathrm{l}$ (0.49-2.31) (Wilcoxon signed rank test $\mathrm{p}<0.001$ ) (fig 2). Atorvastatin was well tolerated: two patients in the placebo group and five patients in the atorvastatin group discontinued the treatment, predominantly as a result of gastrointestinal upset. One patient taking atorvastatin had an increase in creatine kinase of $>5$ times the upper limit of normal without symptoms of myositis and was withdrawn at the request of the Data Monitoring Committee. There were no cases of rhabdomyolysis.

\section{Coronary artery calcium score}

Atorvastatin did not affect the rate of progression of the coronary artery calcium score (fig 2). Similar results were obtained when the $90 \mathrm{HU}$ threshold was used (42 (SD 73)\%/ year in the atorvastatin group and 29 (SD 37)\%/year in the placebo group, $p=0.24)$. Serum LDL cholesterol concentrations did not correlate with the rate of progression of coronary artery calcification $(\mathrm{r}=0.05, \mathrm{p}=0.62)$.

The rates of change of coronary artery calcium scores were primarily analysed on the logarithms of the scores by random coefficients models. ${ }^{22}$ This showed no difference between the average rates of change in the two treatment arms $(\mathrm{p}=0.18)$. The mean coronary calcium score increased by 0.234 (SE 0.037) log AU/year in the atorvastatin group and 0.167 (SE 0.034) $\log$ AU/year in the placebo group. These figures correspond to a $26 \% /$ year increase in the atorvastatin group and $18 \%$ /year in the placebo group. The geometric mean (adjusted for baseline) is $7 \%$ higher at one year with atorvastatin than with placebo, with $95 \%$ confidence limits ranging from $3 \%$ lower to $18 \%$ higher. Figure 3 summarises the observed annual clanges in cononary calcium scores, calculated from the first to the last visit.

As anticipated in such a modest clinical trial, all cause mortality, cardiovascular mortality or cardiovascular hospitalisation did not differ significantly between the two groups.

\section{DISCUSSION}

We have confirmed that, despite major reductions in serum LDL cholesterol and CRP concentrations, atorvastatin $80 \mathrm{mg}$ daily did not halt the progression, or induce regression, of coronary artery calcification in patients with calcific aortic stenosis. Consistent with recent trials of asymptomatic people," 12 our findings contrast notably with previous observational studies and suggest that the potential beneficial effects on coronary artery calcification have been overestimated.

Previous observational and non-randomised prospective studies" 10 have suggested that reductions in serum LDL cholesterol concentrations decrease the progression of coronary calcification. Not all observational studies, however, have had consistent findings. In the largest observational study of 182 patients, Hecht and colleagues ${ }^{23}$ recently found no difference in the progression of coronary calcium scores in patients who were maintained on lipid-lowering treatment and achieved significant reductions in serum LDL cholesterol concentrations. Observational data may be misleading and prospective randomised controlled trials are necessary to confirm or to refute these interesting preliminary observations. The recent BELLES (Beyond Endorsed Lipid Lowering with EBT Scanning) trial ${ }^{12}$ found no differential effect between pravastatin ( $40 \mathrm{mg}$ daily) and atorvastatin $(80 \mathrm{mg}$ daily) on the progression of coronary artery calcification in 615 hyperlipidaemic postmenopausal women. Study follow up was brief (one year), however, and there was no placebo control group. The St Francis Heart Study" randomly assigned 1005 asymptomatic middle-aged men and women with high coronary artery calcium scores to combination atorvastatin $20 \mathrm{mg}$, vitamin C $1 \mathrm{~g}$, and vitamin E $(\alpha$ tocopherol) $1000 \mathrm{U}$ daily or to matching placebos. After 4.3 years of follow up, the rate of progression of coronary artery calcification did not differ.

We have conducted a double blind randomised controlled trial with helical computed tomography in patients with aortic stenosis. Minimisation technique ensured good matching of the baseline characteristics of the patient population and reproducibility studies confirmed the validity of our repeated assessments. Although documenting very similar rates of progression of coronary calcification to previous studies, 1023 we have not observed a reduction in coronary calcification with intensive lipid-lowering treatment despite more than halving serum LDL cholesterol concentrations.

Statins have been extremely successful in the primary and secondary prevention of cardiovascular disease. Why then have we and others not observed a beneficial effect of statin on coronary artery calcification? Unstable atherosclerotic plaques have a large lipid-rich core, a preponderance of macrophages and foam cells, and a thin fibrous cap containing few smooth muscle cells. ${ }^{24}$ It has been suggested that calcified lesions may be relatively more stable, ${ }^{25}$ indicating a possible protective role of calcification in coronary plaques. Statins produce many of their beneficial effects through plaque stabilisation. In both primate ${ }^{26}$ and swine $e^{27}$ models, antiatherosclerotic interventions are associated with an increase in vascular fibrous tissue and calcification. This calcium deposition continues during the initial phase of plaque regression due to the death of foam cells and an increase in necrotic tissue. Thus, vascular calcification may have a role in the initial stabilisation of atherosclerotic plaques. This is consistent with our findings and would account for the lack of effect on the progression of coronary artery calcification despite a reduction in serum CRP concentrations.

After the initial stabilisation of the atherosclerotic plaque, subsequent progression of coronary calcification would be anticipated to be inhibited. The present study was brief, and follow up was only continued for a median of two years. It would be important to extend our observations to five or more years to assess properly the impact of statin on the long-term progression of coronary artery calcification. It should be acknowledged, however, that the clinical benefits of statin are apparent within the first few years, ${ }^{t-\infty}$ and in some cases the first few months, ${ }^{2 s}$ of treatment. Moreover, the St Francis Heart Study showed no beneficial effects despite 4.3 years of follow up."

On the basis of previous non-randomised studies, ${ }^{10}$ the practice of performing serial computed tomography to monitor disease progression and the response to treatment has become widespread, especially in North America. Our data, and those of the St Francis Heart Study" and the BELLES study, ${ }^{12}$ indicate that repeated scanning to assess response to statin is not justified. Indeed, the radiation dose incurred for such serial scans poses potential health risks, particularly when multidetector computed tomography scanners are used.

\section{Study limitations}

Several factors should be taken into account when considering the results of our study. This was a substudy of the SALTIRE trial' that recruited only patients with calcific aortic stenosis. Our findings are consistent, however, with two 
recent randomised controlled trials in asymptomatic younger people without valvular heart disease." 12 Our study therefore suggests that failure of statins to restrict the progression of coronary artery calcification can be extended to include patients with valvular heart disease as well as older populations. Moreover, our findings suggest that the lack of benefit seen in the St Francis Heart Study is not attributable to the modifying effects of antioxidant vitamins.

When compared with electron beam computed tomography, the accuracy of helical computed tomography in detecting coronary artery calcification has been questioned. ${ }^{1 * 29}$ Technological advances have also meant tha double helical scanners have now been overtaken by 64-slice scanners. At trial inception, the double helix scanner was the latest technology, and it would have been inappropriate to replace the scanner during the conduct of the trial. Moreover, our approach has been previously validated ${ }^{21}$ and we have shown good reproducibility of coronary artery calcification scores in patients with scores of $>100 \mathrm{AU}$. We do not believe the absence of a major beneficial effect on coronary artery calcification is attributable to our methods. We acknowledge that our population size is modest; however, the $95 \%$ confidence intervals can exclude a relative reduction in progression of coronary artery calcification of $>3 \%$ /year. We therefore suggest that if lipid-lowering treatment does reduce the progression of coronary artery calcification then the effect is rather small.

The method of quantification of coronary artery calcification is controversial. The Agatston method is traditionally used but this may overestimate the coronary calcium score in newer generation scanners with reduced slice thickness due to partial voluming. More recent methods include the volume $^{30}$ and the coronary calcium mass ${ }^{31}$ scores, although neither is superior to the Agatston score in terms of reproducibility from consecutive scans in an individual patient."

\section{Conclusion}

We conclude that intensive lipid-lowering treatment does not halt the progression, or induce regression, of coronary artery calcification. Although coronary artery calcium scores corre late well with the presence of atherosclerosis and predict future coronary risk, our findings confirm that monitoring progression of coronary artery calcification to assess the response to lipid-lowering treatment has no role.

\section{Authors' affiliations \\ E S Houslay, S J Cowell, N A Boon, D E Newby, Department of}

Cardiology, Royal Infirmary, Edinburgh, UK

R J Prescott, Public Health Sciences, University of Edinburgh, Medical School, Edinburgh, UK

J Reid, J Burton, Department of Radiology, Borders General Hospital, Melrose, Roxburghshire, UK

D B Northridge, Department of Cardiology, Western General Hospital, Edinburgh, UK

The SALTIRE trial was supported by a project grant from the British Hear Foundation (PG/2000/044) and an unrestricted educational grant from Pfizer (UK). Additional support was provided by the Wellcome Trust Clinical Research Facility, Edinburgh.

Competing interests: DEN and NAB hold unrestricted educational gran awards from Pfizer (UK) Ltd. DEN, DBN and NAB have undertaken paid consultancy and served on advisory boards for Pfizer (UK) Ltd.

Author contributions: ESH, SJC, JB and JR acquired the data. ESH and $\mathrm{RP}$ analysed the data. DEN, DBN and NAB conceived and designed the study. All authors contributed to the writing, revision and approval of the paper.

SALTIRE research team: Lorraine Anderson, Corrine Bell, Margaret Bland, Peter Bloomfield, Sharon Cameron, Nicholas Cruden, Jean Cunningham, Hayley Cuthbertson, Laura Flint, Margaret Henderson,
Dawn Lyle, Maureen O'Donnell, Finney Paterson, Karen Paterson, Robin Prescott, Simon Robinson, Heather Spence, Julie Ticknal and Audrey White

\section{REFERENCES}

1 Pletcher MJ, Tice JA, Pignone M, ef al. Using the coronary artery calcium score to predict coronary heart disease events: a systematic review and meto analysis. Arch Intern Med 2004;164:1285-92

2 Raggi P, Cooil B, Shaw U, ef al. Progression of coronary calcium on serial electron beam tomogrophic scanning is greater in patients with future myocardial infarction. Am J Cardiol 2003:92:827-9.

3 Shepherd J, Cobbe SM, Ford I, et al. Prevention of coronary heart disease with pravastatin in men with hypercholesterolemia. West of Scotland Coronary Prevention Study Group. N Engl J Med 1995;333:1301-7.

4 Downs JR, Clearfield $M$, Weis S, et al. Primary prevention of acute coronary events with lovastatin in men and women with average cholesterol levels: results of AFCAPS/TexCAPS. Air Force/Texas Coronary Atherosclerosis. Prevention Study. JAMA 1998;279:1615-22.

5 Scandinavian Simvastatin Survival Study Investigators, Randomised trial of cholesterol lowering in 4444 patients with coronary heart disease: the cholesterol lowering in 4444 patients with coronary heart disease: the
Scandinavian Simvastatin Survival Study (4S). Lancet 1994:344:1383-9.

6 Scandinavian Simvastatin Survival Study (4S). Lancet 1994;344:1383-9. events in older patients with myocardial infarction and cholesterol levels in the average range. Results of the Cholesterol and Recurrent Events (CARE) trial. Ann Intern Med 1998;129:681-9.

7 LIPID Study Group. Prevention of cardiovascular events and death with pravastatin in patients with coronary heart disease and a broad range of initial cholesterol levels. The Long-Term Intervention with Pravastatin in

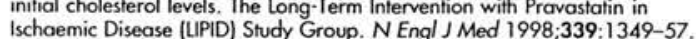

8 Medical Research Council, British Heart Foundation. MRC/BHF Heart Protection Study of cholesterol lowering with simvastatin in 20,536 high-risk individuals: a randomised placebo-controlled trial. Lance 2002;360:7-22.

9 Callister TQ, Raggi P, Cooil B, et al. Effect of HMG.CoA reductase inhibitors on coronary artery disease as assessed by electron-beam computed tomography. N Engl J Med 1998;339:1972-8.

10 Achenbach S, Ropers D, Pohle K, et al. Influence of lipid-lowering therapy on the progression of coronary artery calcification: a prospective evaluation. Circulation 2002;106:1077-82

11 Arad $Y$, Spadaro LA, Roth M, et al. Treatment of asymptomatic adults with elevated coronary calcium scores with atorvastatin, vitamin $C$, and vitamin $E$ the St. Francis Heart Study randomized dinical trial. J Am Coll Cardiol 2005; $46: 166-72$

12 Raggi P, Davidson M, Callister TQ, et al. Aggressive versus moderate lipidlowering therapy in hypercholesterolemic postmenopausal women: Beyond Endorsed Lipid Lowering with EBT Scanning (BELLES). Circulation 2005;112:563-71.

13 Cowell SJ, Newby DE, Prescott RI, et al. A randomized trial of intensive lipid-lowering therapy in calcific aortic stenosis. NEngl J Med 2005;352:2389-97.

14 Rosenhek R, Binder T, Porenta G, ef ol. Predictors of outcome in severe, asymptomatic aortic stenosis. N Engl J Med 2000;343:611-7.

15 Zoghbi WA, Enriquez-Sarano M, Foster E, et al. Recommendations for evaluation of the severity of native valvular regurgitation with two-dimensional and Doppler echocardiography. J Am Soc Echocardiogr 2003;16:777-802.

16 Treasure T, MacRae KD. Minimisation: the platinum standard for trials? Randomisation doesn't guarantee similarity of groups; minimisation does. BM 1998;317:362-3.

17 Carr JJ, Crouse JR 3rd, Goff DC Jr, et al. E. aluation of subsecond gated helical $\mathrm{CT}$ for quantification of coronary artery calcium and comparison with electron beam CT. AUR Am J Roentgenol 2000;174:915-21.

18 Agatston AS, Janowitz WR, Hildner FJ, et al. Quantification of coronary artery calcium using ultrafast computed tomography. I Am Coll Cardiol 1990;15:827-32.

19 Shemesh J, Apter S, Rozenman J, et al. Calcification of coronary arteries: detection and quantification with double-helix CT. Radiology 1995; 197:779-83.

20 Goldin JG, Yoon HC, Greaser LE 3rd, ef al. piral versus electron-beam CT for coronary artery calcium scoring. Radiology 2001;221:213-21.

21 Bland JM, Altman DG. Statistical methods for assessing agreement between two methods of clinical measurement. Lancet 1986;i:307-10.

22 Brown H, Prescolt R. Applied mixed models in medicine. Chichester: John Wiley and Sons, 1999.

23 Hecht HS, Harman SM. Relation of aggressiveness of lipid-lowering treatment to changes in calcified plaque burden by electron beam tomography. Am J Cardiol 2003;92:334-6.

24 Davies M. The composition of coronary-artery plaques. N Engl J Med 1997;336:1312-4.

25 Mintz GS, Popma JJ, Pichard AD, et al. Patterns of calcification in coronary artery disease: a statistical analysis of intravascular ultrasound and coronary angiography in 1155 lesions. Circulation 1995;91:1959-65.

26 Stary HC. The development of calcium deposits in atherosclerotic lesions and their persistence after lipid regression. Am J Cardiol 2001:88(2A):16E-QE.

27 Dooud AS, Jarmolych J, Augustyn JM, ef al. Sequential morphologic studies of regression of advanced atherosclerosis. Arch Pathol lab Med 1981;105:233-9. 
28 Schwartz GG Oliver MF, Ezekowitz MD, et al. Rationale and design of the Myocardial Ischemia Reduction with Aggressive Cholesterol Lowering (MIRACL) study that evaluates atorvastatin in unstable angina pectoris and in non-Q-wave acute myocardial infarction. Am J Cardio 1998;81:578-81.

29 Qanadli SD, Mesurolle B, Aegerter P, et al. Volumetric quantification of coronary artery calcifications using dual-slice spiral CT scanner: improved reproducibility of measurements with 180 degrees linear interpolation algorithm. J Comput Assist Tomogr 2001;25:278-86.
30 Callister TQ, Cooil B, Raya SP, et al. Coronary artery disease: improved reproducibility of calcium scoring with an electron-beam $\mathrm{CT}$ volumetric method. Radiology 1998:208:807-14

31 Hong C, Becker CR, Schoepf UJ, et al. Coronary artery calcium: absolute quantification in nonenhanced and contrast-enhanced multi-detector row $\mathrm{CT}$ studies. Radiology 2002;223:474-80

32 Rumberger JA, Kaufman L. A Roselta stone for coronary calcium risk stratification: Agatston, volume, and mass scores in 11,490 individuals. AUR Am J Roentgenol 2003;181:743-8.

\section{IMAGES IN CARDIOLOGY}

doi: $10.1136 /$ hrt 2005.078733 Anomalous origin of right coronary artery from the mid left anterior descending coronary artery

A 59-year-old women underwent diagnostic coronary angiography with a history of atypical chest pain and an inconclusive treadmill exercise tolerance test. Cine-angiogram revealed an unusual origin of the right coronary artery (RCA) arising from the mid left anterior descending coronary (LAD) artery and coursing to the right, anterior to the right ventricular outflow tract. Such an anomaly is unusual and has not been listed in the classification of such anomalies.

Coronary anomalies are seen in about $1 \%$ of cineangiograms. While some anomalies have been associated with adverse clinical outcomes, most are benign. The RCA has been documented to have an anomalous origin from the left anterior coronary sinus and

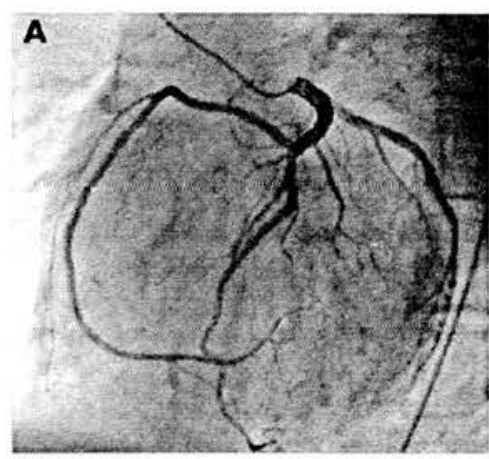

pulmonary trunk, but the origin of the RCA from the LAD has not been reported before.

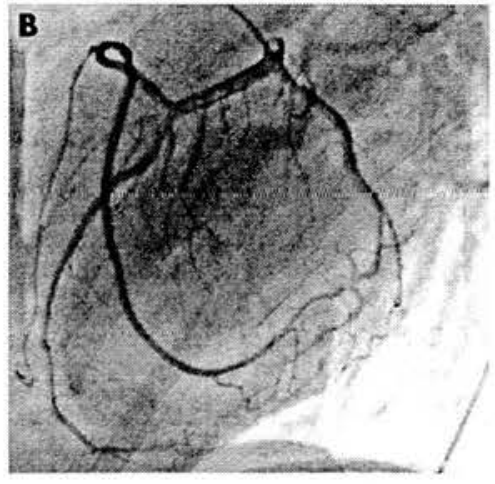

P Saravanan, P Mennim, J E Hancock saravananpl@aol.com

Huge left atrial thrombus in a patient with mitral bioprosthesis

A

77-year-old woman had suffered from atrial fibrillation and rheumatic mitral stenosis for more than 20 years. She underwent mitral valve replacement with bioprosthesis six months before her admission. Inadequate anticoagulation treatment was noted during the follow-up period. She presented with unsteady gait and dizziness to our emergency room. Brain magnetic resonance images confirmed cerebellar infarction. Echocardiography was arranged to search for the possible embolus source, and revealed a huge left atrial thrombus. Because of the thrombus burden and recent stroke, redo cardiac surgery was proposed three weeks after the cerebrovascular event. The preoperative computed tomography (CT) for redo surgery found a large left atrial mass (left panel). During the less invasive cardiac surgery via right small thoracotomy, transoesophageal echocardiography

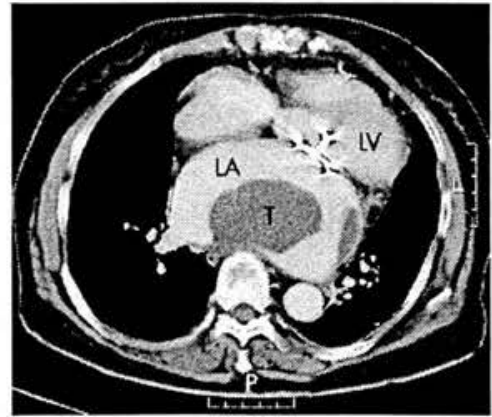

Computed tomography demonstrating a huge mass in an enlarged left atrium. LA, left atrium, LV, left ventricle; T, thrombus.

revealed the significant thrombus burden again (right panel). The bioprosthesis was found to be functioning well and thrombus-free. Additional left atrial appendage closure and endocardial

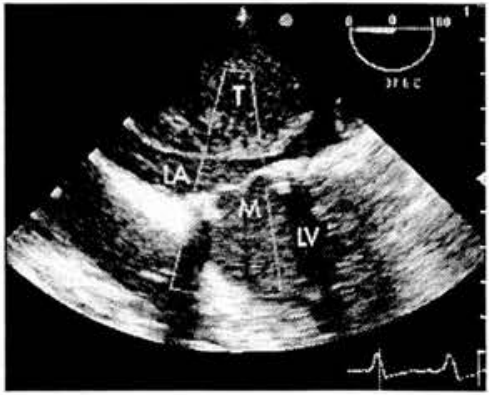

Transoesophageal echocardiography revealing a heavy thrombus burden in the posterior wall of left atrium with the bioprosthesis in the mitral position (M).

ablation were performed to reduce the risk of future thromboembolism

K-M Chiv, T-Y Lin, S-H Chu kmchius@yahoo.com.tw 


\title{
HEART REVIEW
}

\section{Emerging medical treatments for aortic stenosis: statins, angiotensin converting enzyme inhibitors, or both?}

\author{
D E Newby, S J Cowell, N A Boon
}

Aortic stenosis is the most common adult heart valve condition seen in the Western world and its incidence continues to rise. No established disease modifying treatments retard progression of the stenotic process. Recent insights into the pathogenesis of calcific aortic stenosis suggest that the disease mimics atherosclerosis. The natural history and progression of calcific aortic stenosis are described with particular emphasis on new and emerging medical treatments that may modify the disease process. In particular, statins and angiotensin converting enzyme inhibitors appear to hold promise but definitive evidence from large clinical trials is awaited.

See end of article for authors' affiliations

\section{Correspondence to:} Professor David E Newby, Room SU314, Chancellor's Building, Royal Infirmary, 49 Little France Crescent, 49 Little France Crescent,
Edinburgh EHI6 4 SB, UK; d.e.newby@ed.ac.uk

Accepted 13 August 2005
A ortic stenosis is the most common adul heart valve condition seen in the Western world. It is predominantly due to "degenerative" calcific disease, although it can be a consequence of congenital disease such as a bicuspid aortic valve and rheumatic heart disease or of a rare metabolic disease such as ochronosis Watchful waiting and the judicious use of aortic valve replacement surgery remains the mainstay of its management and treatment. We describe here the aetiology and natural history of calcific aortic stenosis and discuss the prospect of developing medical treatments that can modify the disease process.

\section{NATURAL HISTORY OF CALCIFIC AORTIC STENOSIS}

Calcific aortic stenosis has been recognised for over a century. Recently it has been suggested that aortic sclerosis may be the earliest manifestation of this disease process: sclerosis arising from the development of valvar calcific lesions that progress slowly over many years before ultimately causing aortic stenosis.' The current prominence of calcific aortic valve disease probably results from increased human longevity associated with the declining prevalence of rheumatic fever.

Calcific aortic stenosis is a progressive condition, characterised by a long asymptomatic phase lasting several decades, followed by a shorter symptomatic phase usually associated with severe narrowing of the aortic valve orifice. The outlook for patients with asymptomatic disease is generally good but the prognosis changes dramatically with the onset of symptoms in association with severe outflow obstruction-a two year survival rate of about $50 \%$. $^{2}$
Heart 2006;92:729-734. doi: 10.1136/hrt.2005.066852

Despite the favourable outlook for patients with mild asymptomatic disease, the risk of cardiovascular events unrelated to the aortic valve disease is increased. Otto $e t a l^{2}$ reported that patients with aortic sclerosis have a $50 \%$ increased risk of myocardial infarction and cardiovascular death even in the absence of significant outflow tract obstruction. The Helsinki aging study also indicated that patients with moderate to severe aortic stenosis were at an increased risk of all cause and cardiovascular death irrespective of associated symptoms.'

\section{PATHOLOGY OF CALCIFIC AORTIC STENOSIS}

Historically, calcific aortic stenosis has been attributed to prolonged "wear and tear" and age associated valve degeneration. However, recent evidence suggests that it may be the result of an active inflammatory process involving biochemical, humoral, and genetic factors.

Normal aortic valve leaflets appear smooth, thin, and opalescent, with clearly defined tissue layers and very few cells. Increasing age gives rise to thickening of the tips of the valve leaflets, with an increase in the number of adipose cells and thinning of tissue layers. ${ }^{4}$ Calcific aortic stenosis is characterised by leaflet thickening, with irregular nodular masses on the aortic aspect of the valve. Microscopic assessment of both mild and severely affected valves shows endothelial and basement membrane disruption, with underlying subendothelial thickening. The lesion itself contains disorganised collagen fibres, chronic inflammatory cells, lipids, extracellular bone matrix proteins, and bone mineral. ${ }^{4}$

The histological features described closely resemble those seen in atherosclerosis and are strongly suggestive of chronic inflammation (fig 1). The factors initiating the inflammatory process have not been identified but mechanical injury to the endothelium is thought to pave the way for subsequent inflammation. Indeed, the disease tends to affect the aortic surface of the leaflets and the non-coronary cusp that correspond to areas of low shear and high tensile stress. Congenitally bicuspid aortic valves are less efficient than tricuspid valves at distributing mechanical stress leading to the more rapid development of stenosis.

Abbreviations: $A C E$, angiotensin converting enzyme; ASTRONOMER, aortic stenosis progression observation: measuring effect of rosuvastatin; LDL, low density lipoprotein; SALTIRE, Scoltish aortic stenosis and lipid lowering therapy, impact on regression; SEAS, simvastatin and ezetimibe in aortic stenosis 


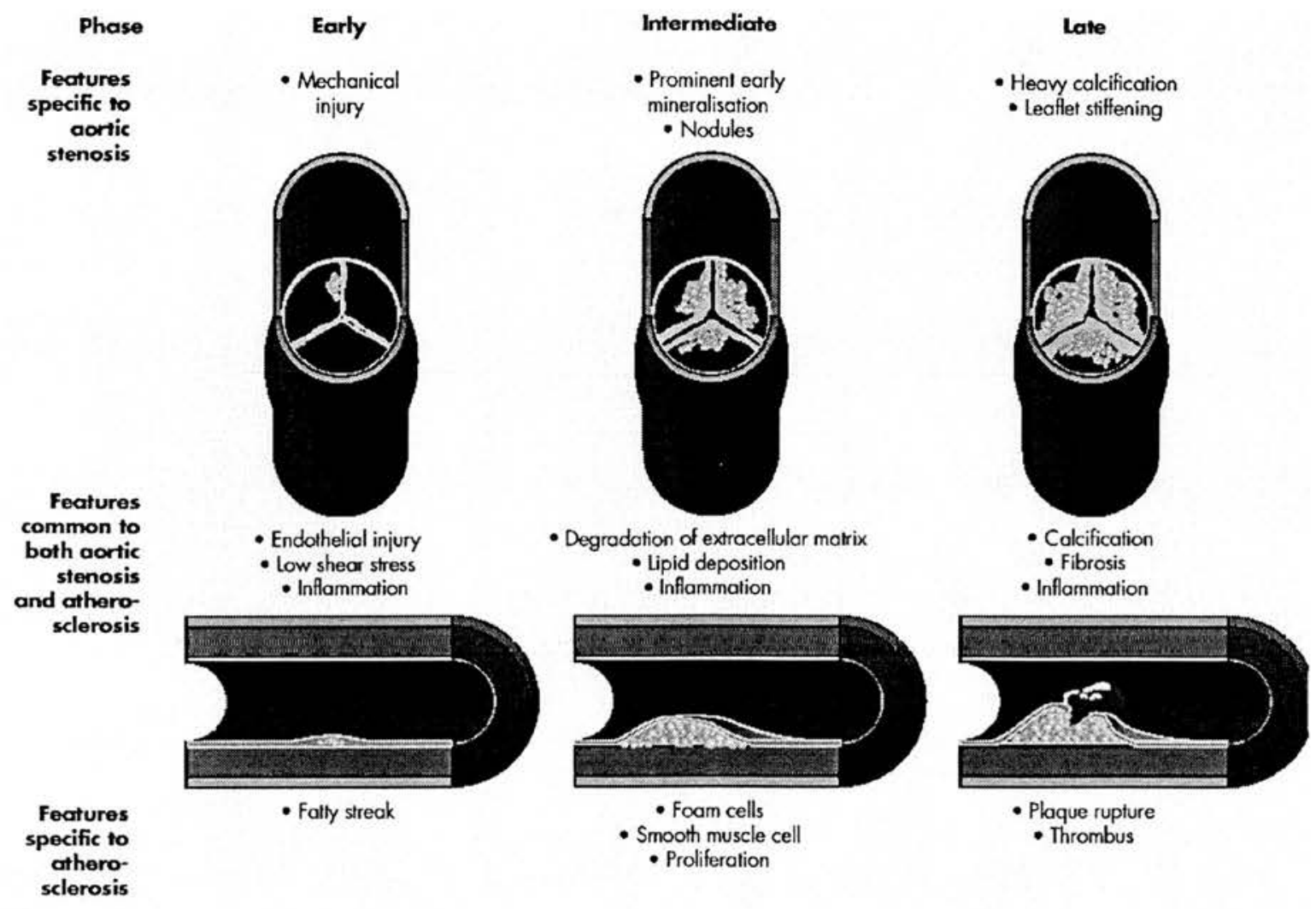

Figure 1 Common and specific pathogenetic features of aortic stenosis and atherosclerosis.

\section{Lipids}

Endothelial injury or disruption may allow lipids to penetrate the valvar interstitial tissue and accumulate in areas of inflammation. ${ }^{56}$ The lipoproteins implicated in atherogenesis, including low density lipoprotein (LDL) and $\mathrm{Lp}(\mathrm{a})$ lipoprotein, are present in early aortic valve lesions and undergo oxidative modification. ${ }^{56}$ These oxidised lipoproteins are highly cytotoxic and capable of stimulating inflammatory activity and mineralisation.?

\section{Inflammation and calcification}

Both macrophages and activated $\mathrm{T}$ lymphocytes are present in the early and advanced lesions of congenitally bicuspid and tricuspid aortic valves. ${ }^{4}$ Migration of these effector inflammatory cells appears to be mediated through increased endothelial expression of cellular adhesion molecules such as E selectin, intercellular adhesion molecule 1 , and vascular cell adhesion molecule 1 . Once recruited into the subendothelium, the inflammatory cells release enzymes, such as matrix metalloproteinases, that degrade collagen, elastin, and proteoglycans within the aortic valve cusps.

Mineralisation is a characteristic of both atherosclerotic and aortic valve lesions and arises close to areas of inflammation. It is a prominent feature in calcific aortic stenosis and has been observed in early as well as advanced lesions. ${ }^{*}$ Surgically excised valves have even shown areas of mature lamellar bone, haemopoietic marrow, and bone remodelling. . Some features suggest the presence of an active highly regulated process closely resembling developmental bone formation.'

The initiation of mineralisation (nucleation) may be stimulated by the presence of cellular degradation products following apoptosis or by the presence of oxidised lipids. " " In vitro studies of cultured explants of stenotic valves have identified cells with osteoblastic characteristics capable of phenotypic differentiation and spontaneous calcification. Their origin is unknown but they may be derived from a pool of circulating immature pluripotent mesenchymal cells. These osteogenic cells or "calcifying valvar cells" express and produce a variety of regulatory bone matrix proteins including osteopontin and bone morphogenetic protein. ${ }^{*}$

\section{CALCIFIC AORTIC STENOSIS AND \\ ATHEROSCLEROSIS}

Although the similarities with atherosclerosis were recog nised as long ago as 1917, they were largely disregarded until recently. Histological studies have highlighted the common features but also confirmed differences in the cellular and mineral components of the two lesions.

Smooth muscle proliferation and lipid laden macrophages (or foam cells) are prominent features of vascular atheroma but are virtually absent from stenotic aortic valves (fig 1). Furthermore, mineralisation occurs earlier and is a more extensive feature of aortic valve lesions than in atherosclerosis. ${ }^{4}$ These differences may, in part, explain why only $40 \%$ of patients with severe aortic stenosis have significant coronary artery disease and why the majority of patients with coronary artery disease do not have aortic stenosis. ${ }^{10}$ As the underlying pathological processes of the two conditions appear to be similar, other unknown factors are likely to influence the development of valvar as opposed to vascular lesions." 
A

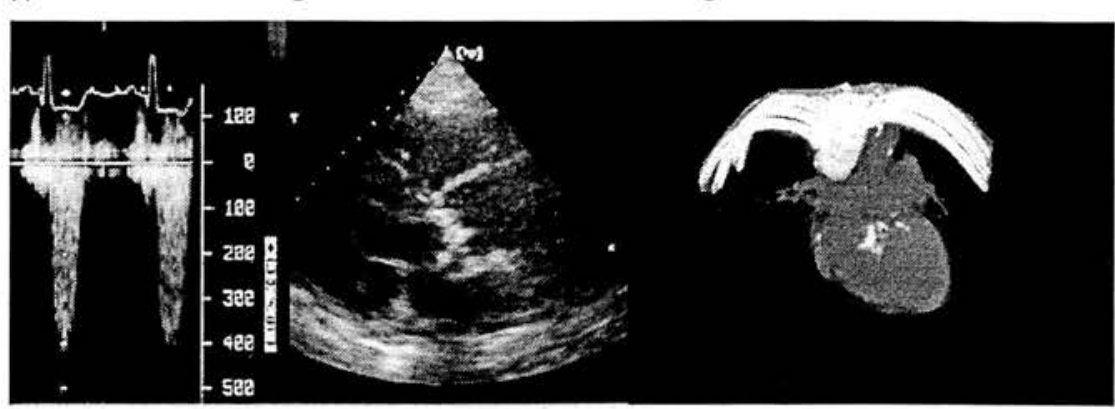

Figure 2 Clinical assessment by (A) Doppler echocardiography; (B) two dimensional echocardiography (parasternal short axis view); and (C) three dimensional computed tomography of the severity (lower panel) of aortic stenosis.

\begin{tabular}{lcccc}
\hline Aortic valve & Normal & Mild & Moderate & Severe \\
\hline Peak velocity $(\mathrm{m} / \mathrm{s})$ & $1-2$ & $2-3$ & $3-4$ & $>4$ \\
Peak gradient $(\mathrm{mm} \mathrm{Hg})$ & 0 & $16-36$ & $36-64$ & $>64$ \\
Mean gradient $(\mathrm{mm} \mathrm{Hg})$ & 0 & $<15$ & $15-50$ & $>50$ \\
Valve area $\left(\mathrm{cm}^{2}\right)$ & $>2.0$ & $2.0-1.2$ & $0.8-1.2$ & $<0.8$ \\
\hline
\end{tabular}

\section{PREDICTORS OF DISEASE PROGRESSION AND CLINICAL OUTCOME}

Patients with calcific aortic stenosis should be monitored regularly in the clinic for the development of symptoms: chest pain, breathlessness, and syncope. Progression of the valvar stenosis is principally monitored with Doppler echocardiography, although complementary clinical information can also be obtained from the ECG (left ventricular hypertrophy, heart block), two dimensional echocardiography, and computed tomography. This permits grading of the severity of the aortic stenosis (fig 2).

The natural history of aortic stenosis is for the valve gradient to rise inexorably with time. Disease progression and clinical outcome have been linked to many of the risk factors for calcific aortic stenosis (table 1). However, much of the evidence is conflicting and limited by the retrospective nature of the studies. The most consistent and strongest predictors of disease progression are severity of stenosis at baseline and degree of valvar calcification: the more severe the stenosis and the more heavily calcified the valve, the faster the rate of progression. ${ }^{12}$ is Clinical outcome is also influenced by the degree of valvar calcification, with nearly $80 \%$ of patients with moderate to severe calcification progressing rapidly ( $>0.3 \mathrm{~m} / \mathrm{s} /$ year) either to death or to aortic valve replacement within two years."

\begin{tabular}{l} 
Table 1 Risk factors for calcific aortic stenosis \\
\hline Clinical \\
Age \\
Male sex \\
Smoking \\
Hypertension \\
Diabetes mellitus \\
Coronary artery disease \\
Chronic renal failure \\
Paget's disease \\
Hyperparathyroidism \\
Biochemical \\
Hyperlipidaemia* \\
Hypercalcaemia \\
Raised serum creatinine \\
\hline "Low density lipoprotein, Lp(a) lipoprotein. \\
\hline
\end{tabular}

\section{NOVEL TREATMENTS FOR CALCIFIC AORTIC STENOSIS}

Current management of patients with aortic stenosis comprises monitoring disease progression and ensuring patient awareness of the need for antibiotic prophylaxis against the relatively low risk of infective endocarditis. For those patients with severe symptomatic disease, aortic valve replacement is a priority with conventional medical treatment reserved for symptom control in inoperable cases. However, the majority of patients with aortic stenosis do not have symptoms or an indication for surgery. Are there any interventions that can halt or slow the progression of the disease process? Theoretically, anti-inflammatory and antiproliferative agents would be anticipated to alter the natural history of aortic stenosis. Statins and angiotensin converting enzyme (ACE) inhibitors are two commonly used treatments that have proven secondary preventative benefits in cardiovascular disease and exhibit some of these desirable antiinflammatory and antiproliferative properties.

\section{Statins}

Hydroxymethylglutaryl coenzyme A reductase inhibitors, or statins, are now well established in the primary and secondary prevention of coronary artery disease. Several studies have suggested that these drugs can cause regression of coronary artery disease and reduce the calcific volume of coronary plaques. ${ }^{14}$ Given the clinical association of calcific aortic stenosis with hyperlipidaemia and coronary artery disease, and the striking histological similarities with atheroma, the hypothesis that statins may have the potential to influence disease progression in aortic stenosis is intriguing. ${ }^{15}$

Recent retrospective studies have suggested that statins may delay disease progression in aortic stenosis (table $2^{16-22}$ ) through their lipid lowering and anti-inflammatory actions. ${ }^{16-19}$ These observational data should be interpreted with caution, since none of these studies were prospective randomised trials, serum LDL cholesterol concentrations did not correlate with disease progression, and the statin doses were small. There also appears to be some publication bias with studies reporting negative findings underrepresented in the literature..$^{21}$ 
Table 2 Summary of trials assessing progression of aortic stenosis by repeated echocardiography

\begin{tabular}{|c|c|c|c|c|c|c|c|}
\hline & \multicolumn{7}{|c|}{ Study and reference } \\
\hline & $\begin{array}{l}\text { Aronow } \\
\text { ef all }\end{array}$ & $\begin{array}{l}\text { Novoro } \\
\text { et al }\end{array}$ & $\begin{array}{l}\text { Bellamy } \\
\text { et al }{ }^{18}\end{array}$ & $\begin{array}{l}\text { Rosenhek } \\
\text { ef af }{ }^{19}\end{array}$ & $\begin{array}{l}\text { Samal } \\
\text { ef of }\end{array}$ & $\begin{array}{l}\text { Antonini-Conterin } \\
\text { ef } \text { of }^{\prime}\end{array}$ & Cowell et a $P^{2}$ \\
\hline Design & RO & $\mathrm{RO}$ & $\mathrm{RO}$ & RO & RO & RO & Prospective $\mathrm{RCT}$ \\
\hline Patients & 180 & 174 & 156 & 211 & 112 & 242 & 134 \\
\hline Patients taking statin & 62 & 57 & 38 & 50 & 55 & 121 & 65 \\
\hline Mean age (years) & 82 & 68 & 77 & 70 & 73 & 68 & 68 \\
\hline Mean follow up (months) & 33 & 21 & 44 & 24 & NA & 54 & 25 \\
\hline Total cholesterol (mmol/l) & NA & 5.5 & 5.8 & 5.8 & NA & NA & 5.7 \\
\hline $\begin{array}{l}\text { Correlation of progression with } \\
\text { WL cholesterol }\end{array}$ & NA & Yes/no & No & No & Yes & NA & No \\
\hline Reduced progression with statin & Yes & Yes & Yes & Yes & No & No & No \\
\hline
\end{tabular}

Results of the SALTIRE (Scottish aortic stenosis and lipid lowering therapy, impact on regression) trial were recently reported. It was the first double blind randomised controlled trial of lipid lowering treatment in patients with calcific aortic stenosis. ${ }^{22}$ This trial of 155 patients showed that, although atorvastatin $80 \mathrm{mg}$ daily more than halved serum LDL cholesterol concentrations, it did not halt the progression or induce regression of the valve disease process as measured by Doppler echocardiography or helical computed tomography (fig 3). This occurred despite the association of atorvastatin with major reductions in serum $\mathrm{C}$ reactive protein concentrations (unpublished observations).

Given the data linking aortic stenosis with atherosclerosis and hypercholesterolaemia, why did intensive lipid lowering treatment not halt the progression of calcific aortic stenosis? One potential explanation is that, while these features may drive the initiation of aortic stenosis, disease progression may depend on other factors. The aortic valve is subjected to continuous dynamic mechanical stress, and leaflet plasticity and structure can have an overriding influence, such as with a bicuspid valve. Moreover, in contrast to atherosclerosis, aortic stenosis is associated with a virtual absence of smooth muscle cell proliferation and lipid laden macrophages, and is dominated by earlier and more extensive mineralisation. Decreasing the lipid pool and increasing the fibrous cap may be less relevant to the progression of aortic stenosis than it is for the reduction of atherosclerotic plaque rupture with statins in patients with coronary heart disease.

It may be argued that lipid lowering treatment is unlikely to influence disease progression in the presence of significant aortic stenosis. Patients with aortic velocities below $2.5 \mathrm{~m} / \mathrm{s}$ were excluded from the SALTIRE trial, and intervening at this earlier stage of the disease process may have been more beneficial. However, such patients do not commonly present to routine clinical practice and their identification would potentially require population screening. Moreover, the SALTIRE trial was unable to exclude a modest treatment benefit (a delay in disease progression of $<0.07 \mathrm{~m} / \mathrm{s} /$ year or $<5 \%$ aortic valve calcification/year). Although such modest reductions are unlikely to be meaningful in the majority of older patients, a small decrease in disease progression may be clinically important in younger patients with mild disease who may progress over many years. Indeed, a small preliminary observational study suggests that statins may reduce disease progression in patients with aortic sclerosis. ${ }^{21}$

Statin treatment of patients with aortic stenosis may confer secondary preventative benefits that are independent of its effects on the valve disease process because of the association between aortic stenosis and coronary artery disease. The SALTIRE trial was not powered to assess the benefits of lipid lowering treatment on cardiovascular end points, such as non-fatal and fatal myocardial infarction, but there was a trend in favour of reduced clinical events. Aortic stenosis and sclerosis may be important markers of occult vascular disease and thereby identify patients who would gain from the preventative benefits of statins. Larger clinical end point trials, such as the SEAS (simvastatin and ezetimibe in aortic stenosis) and ASTRONOMER (aortic stenosis progression observation: measuring effect of rosuvastatin) trials, will be able to address this issue.

Lastly, for many patients with aortic stenosis, the first symptom to develop is chest pain, and this precipitates the decision to replace the aortic valve. However, this may be driven by concomitant coronary artery disease rather than progression of valvar stenosis. Previous secondary prevention trials in coronary heart disease have reported a reduction in coronary artery bypass graft surgery rates. ${ }^{2}$ Thus, the larger clinical end point trials of statins in aortic stenosis may suggest a reduction in the rate of valve surgery, but this may be driven by patients with aortic stenosis who undergo combined aortic valve and bypass surgery for symptoms of angina pectoris. If statins truly reduce disease progression then a reduction in isolated aortic valve replacement would be anticipated.

\section{ACE inhibition}

There are several reasons to believe that ACE inhibitors may have a role in the management of patients with aortic stenosis. Firstly, in contrast to normal valves, sclerotic aortic valve tissues demonstrably express angiotensin II and ACE, which may contribute to valve inflammation, calcification, and disease progression. ${ }^{24} 25$ Secondly, the pressure overload induced by aortic stenosis has several effects on the myocardium including left ventricular hypertrophy, apoptosis, and fibrosis. This may accelerate the left ventricular systolic and diastolic dysfunction associated with aortic stenosis. Lastly, blood pressure lowering indirectly reduces the pressure overload of the left ventricle and potentially reduces the mechanical stress and strain on the aortic valve.

Two preliminary observational studies with ACE inhibitors have produced conflicting results. In a retrospective analysis of 211 patients, Rosenhek et al ${ }^{19}$ found that progression of aortic stenosis was not delayed in patients maintained on ACE inhibitors. Furthermore, the presence of hypertension did not appear to influence the outcome. In contrast, O'Brien et $\mathrm{al}^{24}$ found that ACE inhibitor treatment was associated with a $71 \%$ reduction in the progression of aortic valve calcification in 123 patients with aortic stenosis undergoing electron beam computed tomography. However, such retrospective observational data are difficult to interpret and the study findings have wide confidence intervals.

Historically, ACE inhibition was said to be contraindicated in patients with aortic stenosis. This has primarily been due to the concern of invoking profound peripheral vasodilatation that would result in haemodynamic compromise, collapse, and potentially death. However, patients with aortic stenosis 


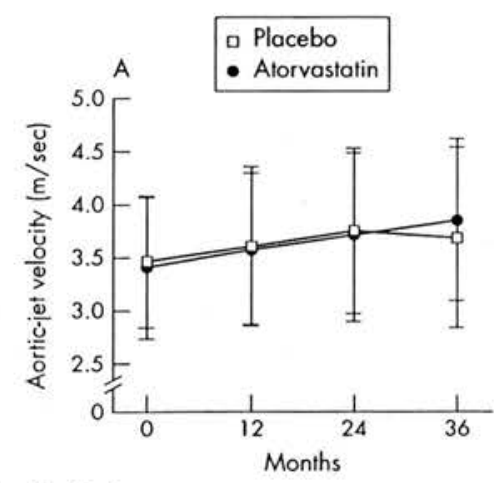

$\begin{array}{lllll}\begin{array}{llll}\text { Number of patients } \\ \text { Placebo }\end{array} & 77 & 69 & 55 & 30 \\ \text { Alorvastatin } & 77 & 65 & 60 & 34\end{array}$
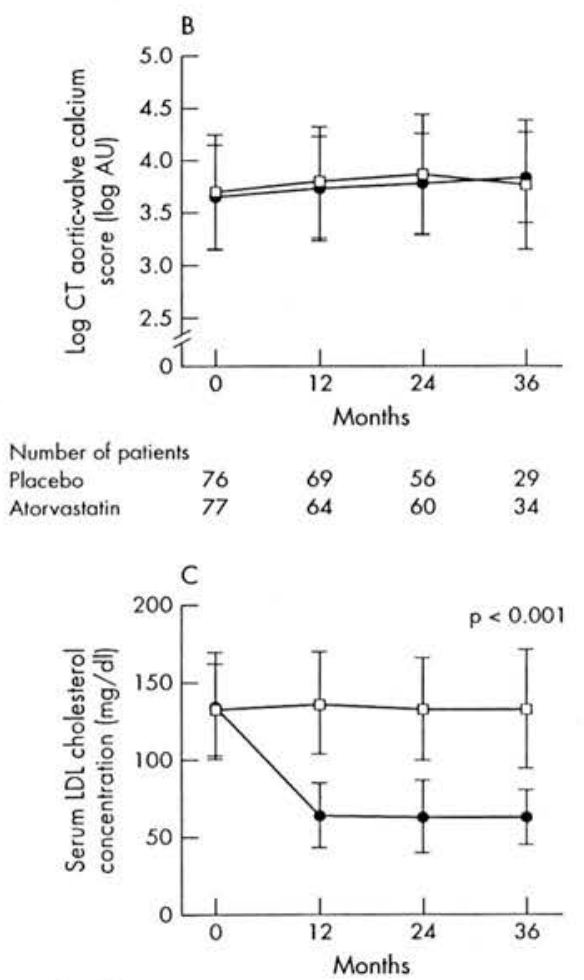

Number of patients

$\begin{array}{lllll}\text { Placebo } & 78 & 70 & 54 & 31\end{array}$

Figure 3 Effect of atorvastatin ( $80 \mathrm{mg}$ daily) on the progression of aortic stenosis and serum low density lipoprotein (LDL) concentrations. ${ }^{22}$ $\mathrm{AU}$, arbitrary units; CT, computed tomography. Copyright ${ }^{\circ} 2005$ Massachusetts Medical Society. All rights reserved. Reproduced with permission of the publisher.

tolerate ACE inhibitors very well on initiation ${ }^{26} 27$ and many patients (about $30 \%$ ) with aortic stenosis are unknowingly established on such treatment without compromise. Indeed, the use of ACE inhibitors appears to confer long term survival benefit on patients considered to have a contraindication including those with aortic stenosis. ${ }^{2 \pi}$ The potential beneficial haemodynamic and cardiac effects of ACE inhibition are increasingly being recognised and warrant further study in patients with aortic stenosis. ${ }^{29}$

\section{CONCLUSIONS}

The need for an alternative to aortic valve surgery is highlighted by the increasing longevity of the population and rising prevalence of aortic stenosis. New therapeutic strategies to limit disease progression are needed to delay, and potentially avoid, the need for valve surgery.

Statins and ACE inhibitors are two potential and promising treatments that may have beneficial effects in patients with aortic stenosis. Statins are likely to reduce cardiovascular events rather than disease progression per se but may potentially be a valuable preventative treatment in these patients. However, we must await the results of ongoing large randomised controlled trials to define the role of statins.

The prejudice against the use of ACE inhibitors by patients with aortic stenosis is changing. ${ }^{23-26}$ We would argue that there is sufficient theoretical evidence to support the conduct of a randomised controlled trial to explore further its potential benefits. In the meantime, the cautious use of ACE inhibition by patients with concomitant hypertension, coronary heart disease, or heart failure seems appropriate.

\section{Authors' affiliations}

D E Newby, S J Cowell, N A Boon, Department of Cardiology, Royal Infirmary, Edinburgh, UK

Conflicts of interest declaration: The authors were involved in the SALTIRE trial funded by the British Heart Foundation with an additional educational grant award from Pfizer (UK) Limited and are participating in the SEAS study, which is sponsored by Merck Sharp and Dohme Limited.

\section{REFERENCES} I Stewart BF, Siscovick D, Lind BK, for the cardiovascular health study, et al.
Clinical factors associated with calcific aortic valve disease. I Am Coll Cardiol 1997:29:630-4

2 Otto CM, Lind BK, Kitzman DW, et al. Association of aortic-valve sclerosis with cardiovascular mortality and morbidity in the elderly. N Engl J Med 1999;341:142-7.

3 livanainen $\mathbf{A M}$, Lindroos $M$, Tilvis $R$, et al. Natural history of aortic valve stenosis of varying severity in the elderly. Am J Cardiol 1996:78.97-101.

4 Otto CM, Kuusisto J, Reichenbach DD, et al. Characterization of the early lesion of 'degenerative' valvular aortic stenosis: histologic and immunohistochemical studies. Circulation 1994:90:844-53.

5 O'Brien KD, Reichenbach DD, Marcovina SM, et al. Apolipoprotein B, (a), and E accumulate in the morphologically early lesion of 'degenerative' valvular aortic stenosis. Arterioscler Thromb Vasc Biol 1996;16:523-32.

6 Olsson M, Thyberg J, Nilsson J. Presence of oxidised low density lipoprotein in nonrheumatic stenotic aortic valves. Arterioscler Thromb Vasc Biol 1999;19:1218-22.

7 Parhami F, Morrow AD, Balucan J, et al. Lipid oxidation products have opposite effects on calcifying vascular cell and bone cell differentiation; a possible explanation for the paradox of arterial calcification in osteoporotic possible explanation for the paradox of arterial calcification
patients. Arterioscler Thromb Vasc Biol 1997;17:680-7.

8 Mohler ER, Gannon F, Reynolds C, et al. Bone formation and inflammation in cardiac valves. Circulation 2001;103:1522-8.

9 Demer $\mathrm{L}$. A skeleton in the atherosclerosis closet. Circulation 1995:92:2029-32.

10 Peltier M, Trojette F, Sarano ME, et al. Relation between cardiovascular risk factors and nonrheumatic severe calcific cortic stenosis among patients with a three-cuspid oortic valve. Am J Cardiol 2003:91:97-9.

11 Otto CM, O'Brien KD. Why is there discordance between calcific aortic stenosis and coronary artery disease? Heart 2001;85:601-2.

12 Otto CM, Burwash IG, Legget ME, et al. Prospective study of asymptomatic valvular aortic stenosis: clinical, echocardiographic, and exercise predictors of outcome. Circulation 1997; 95:2262-70.

13 Rosenhek R, Binder T, Porenta G, ef al. Predictors of outcome in severe, asymptomatic aortic stenosis. N Engl J Med 2000;343:611-7.

14 Callister TQ, Raggi P, Cooil B, ef al. Effect of HMG.CoA reductase inhibitors on coronary artery disease as assessed by electron-beam computed on coronary artery disease as assessed by election
tomography. N Engl J Med 1998;339:1972-8.

15 Mohler ER. Are atherosclerofic processes involved in cortic valve calcification? lancet 2000;356:524-5.

16 Aronow WS, Ahn C, Kronzon I, ef al. Association of coronary risk factors and use of statins with progression of mild valvular aortic stenosis in older persons. Am J Cardiol 2001;88:693-5.

17 Novaro GM, Tiong IY, Pearce GL, et al. Effect of hydroxymethylglutaryl coenzyme a reductase inhibitors on the progression of calcific aortic stenosis. Circulation 2001;104:2205-9. 
18 Bellamy MF, Pellikka PA, Klarich KW, et al. Association of cholesterol levels, hydroxymethylglutaryl coenzyme-A reductase inhibitor treatment and progression of aortic stenosis in the community. J Am Coll Cardiol progression of aortic

19 Rosenhek R, Rader F, Loho N, et al. Statins but not angiotensin-converting enzyme inhibitors delay progression of aortic stenosis. Circulation 2004;110:1291-5.

20 Samal AK, Berman AE, Kuruvanka TS, ef al. Effect of statin therapy in the progression of moderate to severe calcific aortic stenosis. Circulation 2002;106(suppl II):11640.

21 Antonini-Conterin F, Popescu BA, Huang G, et al. Progression of aortic valve sclerosis and aortic valve stenosis: what is the role of statin treatment? Ital Heart J 2005;6:119-24.

22 Cowell SJ, Newby DE, Prescott RJ, et al. A randomized trial of intensive lipid-lowering therapy in calcific aortic stenosis. N Engl J Med lipid-lowering therapy in

23 Heart Protection Study Collaborative Group. MRC/BHF heart protection study of cholesterol lowering with simvastatin in 20,536 high-risk individuals: a randomised placebo-controlled trial. Lancet, 20026, 360:7-22.
$24 \mathrm{O}^{\prime}$ Brien KD, Probstfield Jl, Caulfield MT, et al. Angiotensin-converting enzyme inhibitors and change in aortic valve calcium. Arch Intern Med 2005:165:858-62

25 Helske S, Lindstedt KA, Laine M, ef al. Induction of local angiotensin II-producing systems in stenotic aortic valves. J Am Coll Cardiol 2004:44:1859-66.

26 O'Brien KD, Zhao XQ, Shavelle DM, ef al. Hemodynamic effects of the angiotensin-converting enzyme inhibitor, ramipril, in patients with mild to moderate oortic stenosis and preserved left ventricular function. J Investig Med 2004;52:185-91.

27 Chockalingam A, Venkatesan S, Subramaniam T, et al. Safety and efficacy of angiotensin-converting enzyme inhibitors in symptomatic severe aortic stenosis: symptomatic cardiac obstruction-pilot study of enalopril in cortic stenosis (SCOPE-AS). Am Heart J 2004;147:E19.

28 Ahmed A, Centor RM, Weover MT, et al. A propensity score analysis of the Ahmed A, Centor RM, Weaver MI, ef al. A propensity score analysis of the impact of angiotensin-converting enzyme inhibitors on long-term survival of older odults with heart failure and perceived contraindications. Am Heart 2005; 149:737-43.

29 Routledge HC, Townend JN. ACE inhibition in aortic stenosis: dangerous medicine or golden opportunity? J Hum Hypertens 2001;15:659-67.

\section{IMAGES IN CARDIOLOGY}

\section{A common cause of secondary hypertension: coarctation of the aorta}

A

16 year old male was referred to our department with hypertension refractory to medical treatment. He had recurrent episodes of headache. On physical examination, his blood pressures in right and left arms were $190 / 120$ and $180 /$ $120 \mathrm{~mm} \mathrm{Hg}$, respectively. Also a systolic ejection murmur of grade $2 / 6$ was present at the left upper sternal border radiating to the interscapular region. Femoral pulsations were diminished. The ECG revealed left ventricular hypertrophy. The chest $x$ ray showed no pathology, but echocardiography revealed a bicuspid aortic valve, left ventricular hypertrophy, normal ascending aortic size, and an ejection fraction of $67 \%$ with normal systolic and diastolic dimensions. By using continuous wave Doppler, a $50 \mathrm{~mm} \mathrm{Hg}$ pressure gradient was assessed $3-4 \mathrm{~cm}$ from the left subclavian artery with the suprasternal notch view. Computed tomographic angiography (CTA) of the thoracic aorta was performed. CTA showed a significant coarctation of the thoracic aorta distal to the origin of the left subclavian artery (panel). It was decided to undertake surgical intervention to correct the problem.

Hypertension in teenagers and young adults is uncommon. As secondary causes are more commonly found in this age group than in older adults, aortic coarctation should be considered. Thus, palpation of femoral pulses and measurement of blood pressure in the limbs should be performed in every hypertensive young patient. Early diagnosis and treatment are essential for

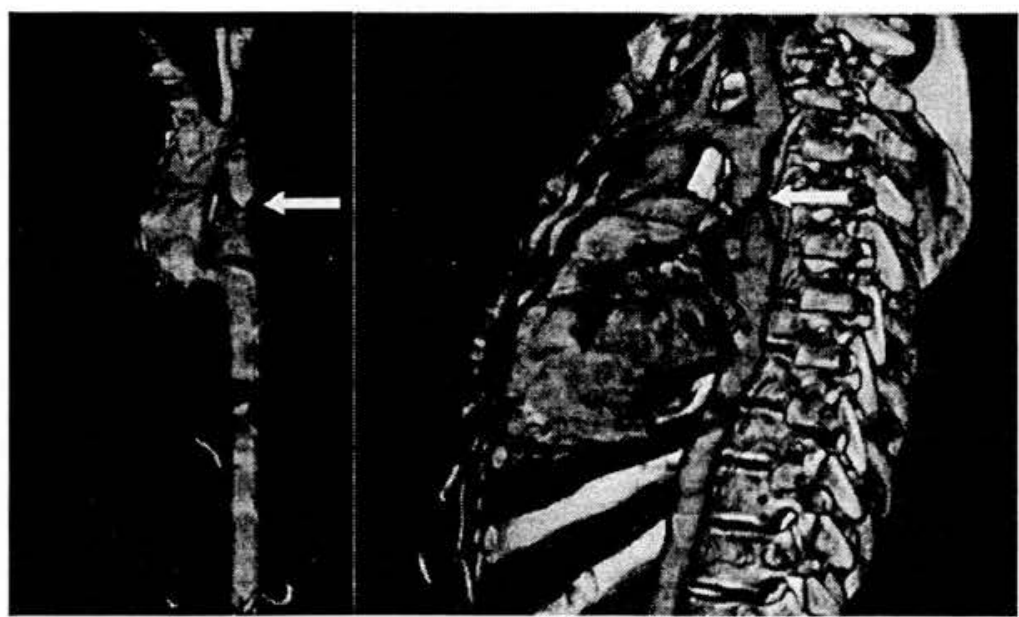

Computed tomographic angiography of the thoracic aorta showing a significant coarctation beyond the origin of the left subclavian artery (arrows).

the prevention of morbidity and mortality from premature cardiovascular complications. Surgical or percutancous techniques should be performed together with medical treatment to prevent end organ damage. 